\title{
Towards Intelligent Playful Environments for Animals based on Natural User Interfaces
}

\author{
by \\ Patricia Pons \\ supervised by \\ Dr. Francisco Javier Jaen
}

\begin{abstract}
A thesis submitted for the degree of
Doctor of Philosophy in Computer Science
\end{abstract}

Departamento de Sistemas Informáticos y Computación

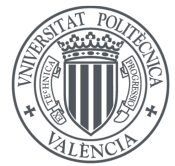

UNIVERSITAT

POLITĖCNICA

DE VALĖNCIA

August 1, 2018 



\section{Summary}

The study of animals' interactions with technology and the development of animalcentered technological systems is gaining attention since the emergence of the research area of Animal Computer Interaction (ACI). ACI aims to improve animals' welfare and wellbeing in several scenarios by developing suitable technology for the animal following an animal-centered approach. Among all the research lines ACI is exploring, there has been significant interest in animals' playful interactions with technology. Technologically mediated playful activities have the potential to provide mental and physical stimulation for animals in different environmental contexts, which could in turn help to improve their wellbeing.

As we embark in the era of the Internet of Things, current technological playful activities for animals have not yet explored the development of pervasive solutions that could provide animals with more adaptation to their preferences as well as offering varied technological stimuli. Instead, playful technology for animals is usually based on digital interactions rather than exploring tangible devices or augmenting the interactions with different stimuli. In addition, these playful activities are already predefined and do not change over time, while they require that a human has to be the one providing the device or technology to the animal. If humans could focus more on their participation as active players of an interactive system aimed for animals instead of being concerned about holding a device for the animal or keep the system running, this might help to create stronger bonds between species and foster better relationships with animals. Moreover, animals' mental and physical stimulation are important aspects that could be fostered if the playful systems designed for animals could offer a varied range of outputs, be tailored to the animal's behaviors and prevented the animal to get used to the system and lose interest.

Therefore, this thesis proposes the design and development of technological playful environments based on Natural User Interfaces that could adapt and react to the animals' natural interactions. These pervasive scenarios would allow animals to play by themselves or with a human, providing more engaging and dynamic playful activities that are capable of adapting over time. 



\section{Resumen}

El estudio de la interacción de los animales con la tecnología y el desarrollo de sistemas tecnológicos centrados en el animal está ganando cada vez más atención desde la aparición del área de Animal Computer Interaction (ACI). ACI persigue mejorar el bienestar de los animales en diferentes entornos a través del desarrollo de tecnología adecuada para ellos siguiendo un enfoque centrado en el animal. Entre las líneas de investigación que ACI está explorando, ha habido bastante interés en la interacción de los animales con la tecnología basada en el juego. Las actividades de juego tecnológicas tienen el potencial de proveer estimulación mental y física a los animales en diferentes contextos, pudiendo ayudar a mejorar su bienestar.

Mientras nos embarcamos en la era de la Internet de las Cosas, las actividades de juego tecnológicas actuales para animales todavía no han explorado el desarrollo de soluciones pervasivas que podrían proveerles de más adaptación a sus preferencias a la vez que ofrecer estímulos tecnológicos más variados. En su lugar, estas actividades están normalmente basadas en interacciones digitales en lugar de explorar dispositivos tangibles o aumentar las interacciones con otro tipo de estímulos. Además, estas actividades de juego están ya predefinidas y no cambian con el tiempo, y requieren que un humano provea el dispositivo o la tecnología al animal. Si los humanos pudiesen centrarse más en su participación como jugadores de un sistema interactivo para animales en lugar de estar pendientes de sujetar un dispositivo para el animal o de mantener el sistema ejecutándose, esto podría ayudar a crear lazos más fuertes entre especies y promover mejores relaciones con los animales. Asimismo, la estimulación mental y física de los animales son aspectos importantes que podrían fomentarse si los sistemas de juego diseñados para ellos pudieran ofrecer un variado rango de respuestas, adaptarse a los comportamientos del animal y evitar que se acostumbre al sistema y pierda el interés.

Por tanto, esta tesis propone el diseño y desarrollo de entornos tecnológicos de juego basados en Interfaces Naturales de Usuario que puedan adaptarse y reaccionar a las interacciones naturales de los animales. Estos entornos pervasivos permitirían a los animales jugar por si mismos o con una persona, ofreciendo actividades de juego más dinámicas y atractivas capaces de adaptarse con el tiempo. 



\section{Resum}

L'estudi de la interacció dels animals amb la tecnologia i el desenvolupament de sistemes tecnològics centrats en l'animal està guanyant cada vegada més atenció des de l'aparició de l'àrea d'Animal Computer Interaction (ACI) . ACI persegueix millorar el benestar dels animals en diferents entorns a través del desenvolupament de tecnologia adequada per a ells amb un enfocament centrat en l'animal. Entre totes les línies d'investigació que ACI està explorant, hi ha hagut prou interès en la interacció dels animals amb la tecnologia basada en el joc. Les activitats de joc tecnològiques tenen el potencial de proveir estimulació mental i física als animals en diferents contextos, podent ajudar a millorar el seu benestar.

Mentre ens embarquem en l'era de la Internet de les Coses, les activitats de joc tecnològiques actuals per a animals encara no han explorat el desenvolupament de solucions pervasives que podrien proveir-los de més adaptació a les seues preferències al mateix temps que oferir estímuls tecnològics més variats. En el seu lloc, estes activitats estan normalment basades en interaccions digitals en compte d'explorar dispositius tangibles o augmentar les interaccions amb estímuls de diferent tipus. A més, aquestes activitats de joc estan ja predefinides i no canvien amb el temps, mentre requereixen que un humà proveïsca el dispositiu o la tecnologia a l'animal. Si els humans pogueren centrar-se més en la seua participació com a jugadors actius d'un sistema interactiu per a animals en compte d'estar pendents de subjectar un dispositiu per a l'animal o de mantenir el sistema executant-se, açò podria ajudar a crear llaços més forts entre espècies i promoure millors relacions amb els animals. Així mateix, l'estimulació mental i física dels animals són aspectes importants que podrien fomentar-se si els sistemes de joc dissenyats per a ells pogueren oferir un rang variat de respostes, adaptar-se als comportaments de l'animal i evitar que aquest s'acostume al sistema i perda l'interès.

Per tant, esta tesi proposa el disseny i desenvolupament d'entorns tecnològics de joc basats en Interfícies Naturals d'Usuari que puguen adaptar-se i reaccionar a les interaccions naturals dels animals. Aquestos escenaris pervasius podrien permetre als animals jugar per si mateixos o amb una persona, oferint activitats de joc més dinàmiques i atractives que siguen capaces d'adaptar-se amb el temps. 



\section{Keywords}

Animal Computer Interaction, Natural User Interfaces, User-Centered Design, Playful Interactions, Interactive Environments, Depth-based Tracking, Robots. 



\section{Agradecimientos}

A lo largo de estos años he contado con el apoyo y cariño de muchísimas personas, a las que me gustaría expresar mi gratitud por haber hecho este trabajo posible.

A mis compañeros de laboratorio, tanto los que continúan aquí como los que ya terminaron, por todos los buenos momentos y el compañerismo que han tenido siempre: Javi, José Antonio, Abel, Priscila, David, Sandra, Ana... En especial a Fernando, Vicente y Alicia, por hacer de cada día una alegría y sacarme siempre una sonrisa, y a Alejandro, por haber sido un gran mentor y un gran apoyo en los primeros años que empecé en el grupo. También quisiera agradecer a Trevor por ayudarme a mejorar cada publicación con sus consejos.

A mis amigos y compañeros de carrera, en especial a Carolina, Raul, Chuel y Joan por estar siempre ahí para escuchar y para levantarme de nuevo. A Marta, Mónica y Sonica, por 24 años llenos de experiencias y confianza, por ser tan valientes y tener siempre palabras de cariño las unas para las otras.

A Kamil, por transmitirme tanta energía, sacarme de mi zona de comfort y hacerme disfrutar de cada pequeño rincón del mundo.

No tengo palabras suficientes para agradecer a mi director de tesis, Javier Jaén, por todo su apoyo, confianza y motivación. No solo es un gran profesional, como docente y como investigador, sino que además es un ejemplo de esfuerzo, capacidad y generosidad. Sin duda, lo mejor de todo este camino, ha sido poder crecer con tus consejos y tu apoyo. Mil gracias por ser, más que un mentor, una gran persona.

Y por último, al pilar más importante de mi vida, mi familia: a mis abuelos, mis tíos, mis primos, por todo su cariño y ánimos. Pero sobretodo, a mis padres, por haberme apoyado en cada paso y en cada decisión, por estar siempre ahí, sin importar cuándo o por qué. Cada paso que doy y cada cosa que logro, es gracias a vosotros, y me siento enormemente afortunada.

Muchísimas gracias a todos. 



\section{Contents}

Acknowledgments $\quad$ xi

Contents $\quad$ xiii

I Preliminaries 1

1 Introduction $\quad 3$

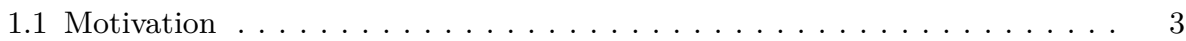

1.1.1 Playful Technology for Animals . . . . . . . . . . . . . . . . . 4

1.1.2 Natural User Interfaces based on Body Tracking. . . . . . . . . . . . . 5

1.2 Research Hypothesis . . . . . . . . . . . . . . . . . 6

1.3 Research Methodology. . . . . . . . . . . . . . . . 6

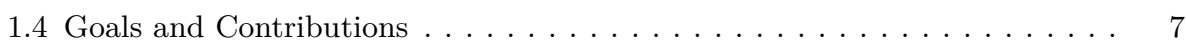

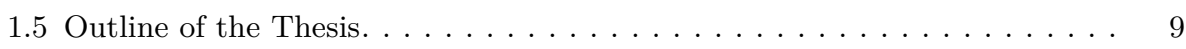

2 Seven years after the Manifesto: Literature Review and Research Directions for Technologies in Animal Computer Interaction $\quad \mathbf{1 5}$

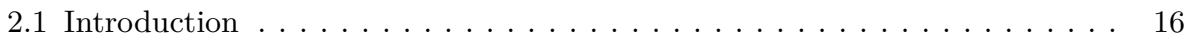



2.2 .1 What is Interaction? . . . . . . . . . . . . . . . . . . 19

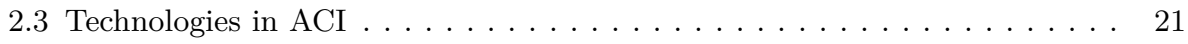

2.3 .1 Model of Technologies within ACI . . . . . . . . . . . . . . . 21

2.3 .2 Tangible and Physical Objects . . . . . . . . . . . . . . . . . 22 
2.3.3 Haptic and Wearable Technologies . . . . . . . . . . . . . . . . . 27

2.3 .4 Olfactory Interfaces . . . . . . . . . . . . . . . . . 31

2.3 .5 Screen Technology . . . . . . . . . . . . . . . . . . . 33

2.3.6 Tracking Technologies . . . . . . . . . . . . . . . . . 37

2.4 Future of Technologies within ACI . . . . . . . . . . . . . . . . . 40

2.4.1 Animal-Driven Devices for Enrichment and Work . . . . . . . . . . . . . . 42

2.4.2 Investigating What Is Interactivity in Animal-Computer Interaction (ACI) 42

2.4.3 Ethics and Agency in Animal-Computer Interaction (ACI) . . . . . . . . 43

2.4.4 Moving Beyond the Human-Animal-Computer Void. . . . . . . . . . . . . . 44

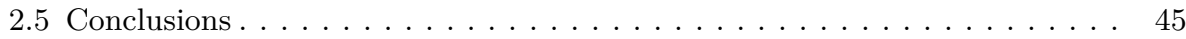

\section{Envisioning Future Playful Interactive Environments for Ani-} mals

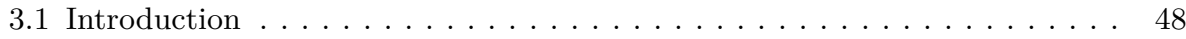

3.1.1 Animals as Target Users of Digital Systems . . . . . . . . . . . . . . . 49

3.1.2 Playful Environments as Intelligent Ecosystems . . . . . . . . . . . . . 51

3.2 Related Works . . . . . . . . . . . . . . . . . . . . . 52

3.2 .1 Computer Interfaces for Animals . . . . . . . . . . . . . . . 52

3.2.2 Playful Experiences within Animal Computer Interaction . . . . . . . . . . 55

3.3 Situating Intelligent Playful Environments . . . . . . . . . . . . . . . . 59

3.4 Situating Current Playful Environments for Animals . . . . . . . . . . . . 62

3.4.1 Game Participants: Static or Dynamic Approach? . . . . . . . . . . . 62

3.4.2 Adapting Computer Interfaces to a Broader Audience: Species Awareness and Interrelationships . . . . . . . . . . . . . . . . . 65

3.4.3 Broadening the Horizon: More Devices, More Fun! . . . . . . . . . . . . . . 66

3.4.4 Decision Making and Adaptation: Who Controls the Controllers? . . . . . . 67

3.5 Application Scenarios for IPE4A . . . . . . . . . . . . . . . . . . 68

3.5.1 Mental Well-being . . . . . . . . . . . . . . . . . 68

3.5 .2 Physical Activity . . . . . . . . . . . . . . . . . . 69



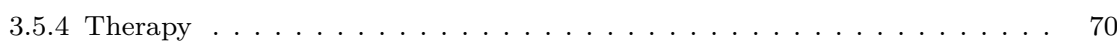

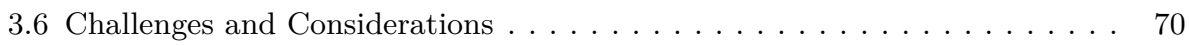

3.7 Conclusions and Future Work . . . . . . . . . . . . . . . . . . . . . 72 
4 Developing a depth-based tracking system for interactive playful environments with animals

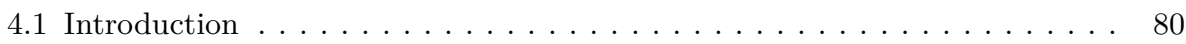

4.2 Related Works . . . . . . . . . . . . . . . . . . . . . 81

4.3 Tracking System. . . . . . . . . . . . . . . . . . . 82

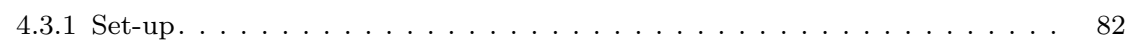

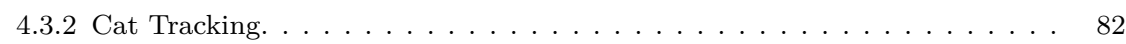

4.3 .3 Human Tracking . . . . . . . . . . . . . . . . . . 85

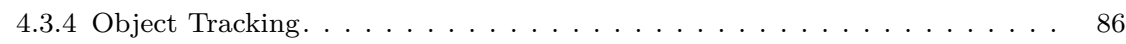

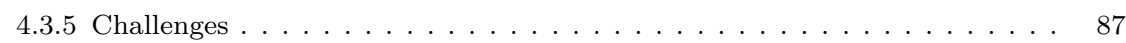

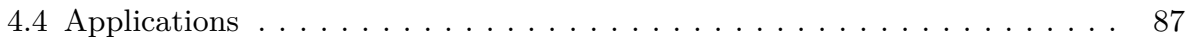

4.4.1 Intelligent Playful Environments based on Gesture and Posture Recogni-

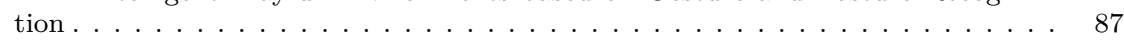

4.4 .2 Learning Behavioral Habits. . . . . . . . . . . . . . . . . . . 89

4.5 Designing Future Tracking-based Gaming Experiences . . . . . . . . . . . . 90

4.5 .1 Tracking Systems for Open Spaces … . . . . . . . . . . . . . . 90

4.5.2 Mixed-reality Games and Embodied Interactions . . . . . . . . . . . . . 91

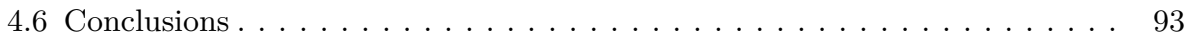

5 Towards Future Interactive Intelligent Systems for Animals: Study and Recognition of Embodied Interactions $\quad 95$

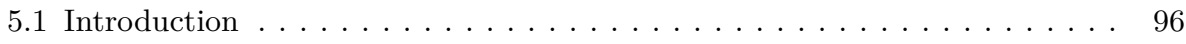

5.2 Related Works . . . . . . . . . . . . . . . . . . . . 98

5.2 .1 Technologically Mediated Playful Systems for Animals . . . . . . . . . . . . 98

5.2.2 Animals' Behavior and Posture Recognition . . . . . . . . . . . . . . . . . 99

5.3 Playful Interactions with Technology . . . . . . . . . . . . . . . . . 100

5.3 .1 Observational Study . . . . . . . . . . . . . . . . . . . . . . . . 100

5.3 .2 Results and Discussion . . . . . . . . . . . . . . . . . . 105

5.4 Behavior Recognition Using Depth-based Tracking. . . . . . . . . . . . . . 108

5.4 .1 System Description. . . . . . . . . . . . . . . . . . . . . . . . 109

5.4 .2 System Evaluation . . . . . . . . . . . . . . . . . . . 112

5.5 Conclusions and Future Work . . . . . . . . . . . . . . . . . . . . . 114 
6 Assessing machine learning classifiers for the detection of animals' behavior using depth-based tracking

6.1 Introduction . . . . . . . . . . . . . . . . . . . . . 118

6.2 Related Works . . . . . . . . . . . . . . . . . . . . . 120

6.2 .1 Wearable Tracking Systems. . . . . . . . . . . . . . . . . . . . . . 120

6.2 .2 Non-wearable Tracking Systems . . . . . . . . . . . . . . . . . . . 122

6.3 Tracking System. . . . . . . . . . . . . . . . . . . . . . . 124

6.3 .1 Equipment and Procedure . . . . . . . . . . . . . . . . 125

6.3 .2 Processing Depth-based Information . . . . . . . . . . . . . . . 126

6.4 Classification Results and Discussion . . . . . . . . . . . . . . . . 127

6.4.1 Supervised Classification of Body Parts and Postures. . . . . . . . . . . . 128

6.4.2 Knowledge-based Classification of Body Parts and Postures. . . . . . . . . 131

6.5 Applications within Animal-Computer Interaction . . . . . . . . . . 135

6.5.1 Behavior Recognition, Learning Behavioral Habits and Welfare Assessment 135

6.5.2 Playful Environments based on Gesture and Posture Recognition . . . . . . 136

6.5.3 Tracking Systems for Zoo Enrichment and Open Spaces . . . . . . . . . . . 138

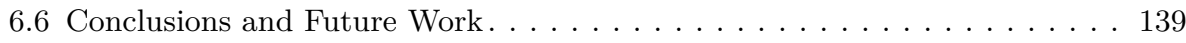

\section{Tangible User Interfaces for Animal Enrichment 143}

7 Tangible User Interfaces for Zoo Enrichment 147

7.1 Introduction . . . . . . . . . . . . . . . . . . . . . . . . . . 148

7.2 Proposal and Research Agenda . . . . . . . . . . . . . . . . . . . . . 149

7.3 Benefits . . . . . . . . . . . . . . . . . . . . . 150



8 Sound to your Objects: A Novel Design Approach to Evaluate Orangutans' Interest in Sound-based Stimuli 153

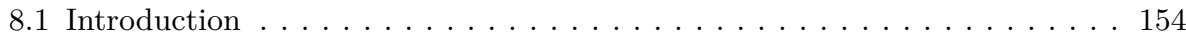

8.2 Related Works . . . . . . . . . . . . . . . . . . . . . . . 155

8.3 Design Process . . . . . . . . . . . . . . . . . . . . . 156

8.3 .1 Tangible Interactions. . . . . . . . . . . . . . . . . . . . 156

8.3 .2 Technical Development . . . . . . . . . . . . . . . . 158

8.3 .3 Sound-based Enrichment . . . . . . . . . . . . . . . . . . . . . . . . 159 
8.4 Conclusion and Future Work . . . . . . . . . . . . . . . . . . . . . . 160

\section{Interspecies Participatory Design of Playful Inter- active Environments}

9 Interactive spaces for children: gesture elicitation for controlling ground mini-robots

9.1 Introduction . . . . . . . . . . . . . . . . . . . . . . . 168

9.2 Related Works . . . . . . . . . . . . . . . . . . . . . . 169

9.2.1 Children's Communication with Robots and Interactive Spaces. . . . . . . 169

9.2 .2 Elicitation Studies with Children . . . . . . . . . . . . . . . . 171

9.2 .3 Elicitation Studies with Adults . . . . . . . . . . . . . . . . . 172

9.3 Elicitation Study . . . . . . . . . . . . . . . . . . . . . . . 173

9.3 .1 Participants . . . . . . . . . . . . . . . . . . . . . 174

9.3 .2 Set-up. . . . . . . . . . . . . . . . . . . . . . . . 174

9.3 .3 Procedure . . . . . . . . . . . . . . . . . . . . . . 175

9.3 .4 Results . . . . . . . . . . . . . . . . . . . . . . . . 177

9.4 Discussion and Future Recommendations . . . . . . . . . . . . . . . 190

9.5 Threats to Validity . . . . . . . . . . . . . . . . . . . . 195

9.6 Conclusion and Future Work . . . . . . . . . . . . . . . . . 195

10 Designing interspecies playful interactions: studying children $\begin{array}{ll}\text { perceptions of games with animals } & 197\end{array}$

10.1 Introduction. . . . . . . . . . . . . . . . . . . . . . . . . 198

10.2 Related Works . . . . . . . . . . . . . . . . . . . . . . . . . . . 199

10.2.1 Participatory Design with Children . . . . . . . . . . . . . . . . 199

10.2.2 ACI Playful Interactions and Human Perceptions . . . . . . . . . . . . 200

10.3 Designing Games for Animals . . . . . . . . . . . . . . . . . . . . . 201

10.3.1 Participants . . . . . . . . . . . . . . . . . . . 201

10.3 .2 Procedure and Materials. . . . . . . . . . . . . . . . . . . . 201

10.3.3 Experimental Design . . . . . . . . . . . . . . . . . . . . . . 203

10.3.4 Observational Findings. . . . . . . . . . . . . . . . . . . . . 204

10.3.5 Design Results . . . . . . . . . . . . . . . . . . . . . . . . . 206

10.3.6 Questionnaire Results . . . . . . . . . . . . . . . . . . . . . . . 213 
10.4 Discussion . . . . . . . . . . . . . . . . . . . . . . . . 217

10.4.1 Improving Relationships . . . . . . . . . . . . . . . . . . . . . . . . 217

10.4.2 Collaboration . . . . . . . . . . . . . . . . . . . . . 218

10.4.3 Remote Interaction . . . . . . . . . . . . . . . . . . . . . . . . . . . 219

10.4.4 Threats to Validity . . . . . . . . . . . . . . . . . . . . . . . . 219

10.5 Conclusion and Future Work. . . . . . . . . . . . . . . . . . . 220

11 Remote Interspecies Interactions: Improving humans and animals' wellbeing through mobile playful spaces

11.1 Introduction. . . . . . . . . . . . . . . . . . . . . . . . . 222

11.2 Related Works . . . . . . . . . . . . . . . . . . . . . . . 223

11.2.1 Applications for Hospitalized Children . . . . . . . . . . . . . . . . 224

11.2.2 Playful Applications for and with Animals. . . . . . . . . . . . . . 224

11.2.3 Designing to Improve Relationships between Humans and Animals. . . . . 225

11.3 Technological Platform . . . . . . . . . . . . . . . . . . 226

11.4 Observational Study on Children . . . . . . . . . . . . . . . . . 228

11.4.1 Participants . . . . . . . . . . . . . . . . . . . . 228

11.4.2 Methodology and Procedure . . . . . . . . . . . . . . 228

11.4.3 Observational Results . . . . . . . . . . . . . . . . . . . . . . . 230

11.4.4 Questionnaire Results . . . . . . . . . . . . . . . . . . . . . 231

11.5 Animal Computer Interaction for Playful Interactive Environments . . . . . 234

11.5.1 Participants . . . . . . . . . . . . . . . . . . . . 235

11.5.2 Interactive Playful Environment for Dogs: Remote vs. Pervasive . . . . . . 235

11.5.3 Observational Results . . . . . . . . . . . . . . . . . . . . . 238

11.5.4 Threats to Validity . . . . . . . . . . . . . . . . . . . . . 239

11.6 Discussion . . . . . . . . . . . . . . . . . . . . . . . . . 240

11.6.1 Remote Mobile Games for Children's Wellbeing . . . . . . . . . . . . . 240

11.6.2 Improving the Human-Animal Relationship . . . . . . . . . . . . . . . . . 242

11.6.3 Shaping the Future of Pervasive Interactive Spaces for Animals . . . . . . . 243

11.7 Conclusion and Future Work. . . . . . . . . . . . . . . . . . . . . . 244

$\begin{array}{llr}\text { V Closure } & 247\end{array}$

12 Discussion $\quad 249$

12.1 On NUIs for the Detection of Animals' Behaviors. . . . . . . . . . . . . . . . 250 
12.2 On NUIs Adaptation to Different Species and Contexts. . . . . . . . . . 252

12.3 On Human Participation in Playful Interactive Spaces for Animals . . . . 254

13 Conclusions and Future Work $\quad 259$

$\begin{array}{lr}\text { Bibliography } & 263\end{array}$

$\begin{array}{ll}\text { Figures } & 305\end{array}$

$\begin{array}{lr}\text { Tables } & 311\end{array}$ 

Part I

\section{Preliminaries}





\section{Chapter 1}

\section{Introduction}

\subsection{Motivation}

Technology no-longer just affects human beings in the ways in which we perceive and interact with our world. With an estimated 80 million European households owning at least one pet animal ${ }^{1}$, and more than $68 \%$ of U.S. households owning a pet ${ }^{2}$, animals are without question a significant population coexisting with our technological surroundings. Domestic dogs and cats have been observed using some of our technological devices, such as smart-phones or tablets, in their own way $[17,224]$ and some zoos have also introduced technology to provide enrichment activities for their animals [46, 240, 252, 343]. Wild animals are also being exposed to our technological innovations with recent trends such as the use of hightech drones for surveillance and monitoring wildlife [91, 170, 336]. Technology is therefore unquestionably changing not only our world and our everyday lives, but also that of the non-human animals ${ }^{3}$ that co-exist with us: from domestic pets to farm or zoo animals, or even wild animals, in one way or another all of them are being exposed to our technological innovations.

However, most of the technology we design and develop is focused on humans' physical characteristics and cognitive understanding of the world [173, 189]. The study of human interactions with technological devices and systems has helped to create ubiquitous spaces and seamless integration of technology into our lives [345]. This in turn has assisted us in our everyday activities, communications and workplaces, and has provided human beings with many advances and benefits derived from the study of Human Computer Interactions (HCI). However, animals have

\footnotetext{
${ }^{1}$ http://www.fediaf.org/who-we-are/facts-and-figures.html

${ }^{2}$ 2017-2018 APPA National Pet Owners Survey

${ }^{3}$ For now on in this thesis, when referring to animal beings we are not including humans in this group, although Homo sapiens is included in the animal kingdom.
} 
different physical features and mental perceptions of the world, preventing them from fully using and understanding our technology and interaction methods, which have been designed with human requirements and characteristics in mind [189]. This puts animals at a disadvantage regarding the benefits technology could bring into their lives: if animals cannot fully explore or interact with technology, they cannot receive the whole spectrum of advantages that technological innovations could bring to their wellbeing $[173,363]$.

A novel and ever-growing research area known as Animal Computer Interaction (ACI) $[173,174]$ is now addressing the aforementioned concerns. ACI focuses on studying animals' interactions with technology following a user-centered approach, i.e. considering animals as the target users of a digital system, based on the development of methodologies, computer interfaces and digital systems specifically designed for animals. The study of animals' interactions with technology and the development of animal-centered technological systems aim to improve animals' welfare and wellbeing in different scenarios: technology for working animals [133, 180, 193, 281], technology for animal welfare [104, 143, 176, 324], or technology for entertainment [142, 258, 343,351], among others.

\subsubsection{Playful Technology for Animals}

Play is one of the most natural and inherent behaviors among human and nonhuman animals. It is also a very important part of every animal's life as it teaches them the types of behavior they need to acquire in their adult life. It also helps to keep their minds and bodies active, reduces stress and could be an indicator of wellbeing [258, 363]. Research within Animal Computer Interaction has shown interest in animals' playful interactions with technology [79, 142, 257, 323, 343, 351]. In this regard, technologically-mediated playful activities have the potential to provide mental and physical stimulation for animals in different environmental contexts.

However, current digital playful activities for animals are mostly mediated by humans: although there are few examples of humans participating in games designed for animals [224, 323, 343, 351], in all the scenarios the human is the one responsible for providing the device or technology to the animal. Animals therefore cannot easily play by themselves or decide when they want to start the activity. Animals would have more opportunities to improve their wellbeing by means of technologically-mediated games if these were not subject to humans providing the activity. In this regard, more efforts could be directed towards allowing animals to decide when they want to play and creating systems that can adapt to their decisions. This would also help to foster human-animal interactions during these technologically mediated experiences, which are beneficial to both sides of the interplay [363]. There is an opportunity in working towards allowing humans to focus more on their participation as active players in an interactive system for ani- 
mals. Instead of being concerned about holding a device for the animal or keeping the system running, humans should rather be fully immersed in the activity, which could help to create stronger bonds between species and foster empathy and better relationships with animals [363]. Likewise, playful systems for animals in the related literature usually follow a predefined flow, i.e. the interaction is already preprogrammed and the system reacts in the same way towards the same input. In this regard, mental and physical stimulation are important aspects that would be fostered if the playful systems designed for animals were to offer a varied range of outputs tailored to the animal's behaviors, preventing the animal from getting tired of the system and losing interest. Moreover, although we are in the era of the Internet of Things, playful technology for animals usually focuses on digital artifacts $[113,323,343,351]$ instead of looking towards more pervasive scenarios or the inclusion of tangible or everyday objects in the game.

With the aim of improving animals' wellbeing by addressing the aforementioned issues, this thesis therefore proposes the design and development of technological playful environments able to adapt and react to the animals' playful interactions, allowing them to play either by themselves or with a human and providing more engaging and dynamic playful activities that can be adapted over time.

\subsubsection{Natural User Interfaces based on Body Tracking}

Animals cannot provide verbal feedback or configure themselves the system they are interacting with in order to adapt it to their preferences [222, 364]. In order to provide meaningful responses from the system while allowing the animal to interact naturally, we need to look at how animals spontaneously interact and communicate with their surroundings. Animals of different species may use different ways to communicate [31, 90, 322], e.g. auditory signals in birds [321] or ants leaving pheromone trails [302]. One of the ways of communication among animals, including humans, is by means of body language $[68,206]$, and these visual signals can also mediate human-animal communication $[82,196]$. In non-technologicallybased games between humans and animals, humans are full participants in the interaction and the game adapts to both players' reactions over time. For example, in a typical catch-the-ball game between a dog and a human, the human adapts the intensity of the activity and knows where to throw the ball depending on where the animal is, how far it is from the ball and how keen it is to keep on playing. This adaptation of both players to the observed reactions of one another is what makes a simple activity amusing and entertaining for both actors: the playful activity is different every time and is tailored to the players' needs at all times during the interaction. Therefore, a promising way of building playful systems for animals could be based on this principle of observing and adapting naturally to the participants' interactions, while allowing the animals either to play by themselves or to play with humans. 
Inspired by the emergence of Natural User Interfaces for human users, this thesis explores the design of Natural User Interfaces for animals that support the development of interactive spaces for them, in which humans could also participate as players and not just as providers of the activity. These NUIs for animals (and human) participants would be based on the study of their body movements, gestures or actions during the playful activity. For this, this thesis resorts to non-wearable tracking systems as they can help to detect animals' spontaneous behavior without the limitations or changes in behavior that the use of wearables might cause [233]. In addition, this tracking approach would allow us to extract information not only from the animal player but also from the rest of the environment, i.e. interactive devices or human participants and their interactions with the animals.

\subsection{Research Hypothesis}

Based on the elements and problems described above, the research hypothesis explored here deals with (1) the technological aspects required to build the approach and (2) the suitability of the solution for either the animals alone or both the animal and human users. It can be expressed as follows:

"Natural User Interfaces can be used effectively to create interactive and intelligent playful spaces for animals and humans, and offer an added value in terms of improving the animals' and humans' wellbeing, user experience and interrelationships".

In this thesis, we will seek to improve animals' wellbeing by exploring the one of the five freedoms ${ }^{4}$ that defines the freedom to express natural behavior, understood as the animals being able to exercise, play and have mental stimulation. The animals' user experience will be explored by the design of user-centered systems aimed at spontaneous animal behavior. To assess the improvement in human wellbeing, psychological and emotional aspects will need to be considered. Human user experiences and interrelationships with animals will be studied by traditional assessment of the tools and systems by means of questionnaires.

\subsection{Research Methodology}

As stated in Section 1.2, this work attempts to explore how NUIs could be used to create intelligent and interactive playful environments for animals, allowing animals to play either by themselves or in mediating human-animal interspecies play. To reach this goal, the Design Science research methodology [327] was applied, as it enables the design and validation of approaches to practical problems. In the words of Hevner et al. [112], "the design-science paradigm seeks to extend

\footnotetext{
${ }^{4}$ https://www.rnzspca.org.nz/animal-welfare/the-5-freedoms
} 
the boundaries of human and organizational capabilities by creating new and innovative artifacts". The Design Science process in Information Systems firstly identifies the problem, sets the objectives of the solution, and then the solution is designed and developed, followed by an evaluation of the outcomes. It was decided to adopt Wieringa's methodological proposal [355], which structures the research methodology in nested sets of problems and tasks. As shown in Figure 1.1, the thesis is composed of different problem descriptions or discussions, system designs, evaluations, and future extensions to the designs.

Due to the interdisciplinary nature of the field, the designs or evaluations of the different contributions cited in Section 1.4 required to apply concepts, tools and methods from different disciplines and research approaches. These include usercentered design [220] to focus on the target user group's needs and requirements, Research through Design (Rtd) [81, 372] in order to build appropriate designs by iteratively observing and adapting to the animals' reactions, and participatory design $[59,207]$ to elicit requirements and co-design with the human participants of these interactive spaces.

\subsection{Goals and Contributions}

The overall aim of this thesis is to advance the development of playful technology for animals by incorporating the approach of NUIs in order to create pervasive and animal-centered interactive environments for animals. Such systems should enable the animals to play with the system by themselves as well as allow interspecies playful activities between humans and animals. These shared interactive scenarios between humans and animals will focus on the use of NUIs to incite natural interactions from all the players allowing them to focus on their interplay, which could help to strengthen their bonds and relationships.

To achieve this goal, several studies are proposed to explore the design and development of intelligent and interactive playful environments for animals based on NUIs (see 1.1). First of all, this thesis explores the definition, applications and benefits of the proposed playful interactive environments for animals. Then, several studies have led to the design and development of a system based on NUIs for animals that could support the design and creation of these suitable interactive spaces. In order to assess the suitability of the proposed approach based on NUIs, two different animal populations and contexts were studied, resulting in an observational study of domestic cats, and a Research through Design approach to propose and implement an interactive system for zoo enrichment with orangutans. In both scenarios, tangible elements were considered as effective for building NUIs capable of tracking and augmenting the interaction with such devices. In the case of domestic animals, as tangible robots showed promise for engaging the animal, they were proposed as suitable devices to be included in a future interactive play- 
DESIGN PROBLEM:

How to build interactive and intelligent playful environments for animals (and humans) based on NUIs?

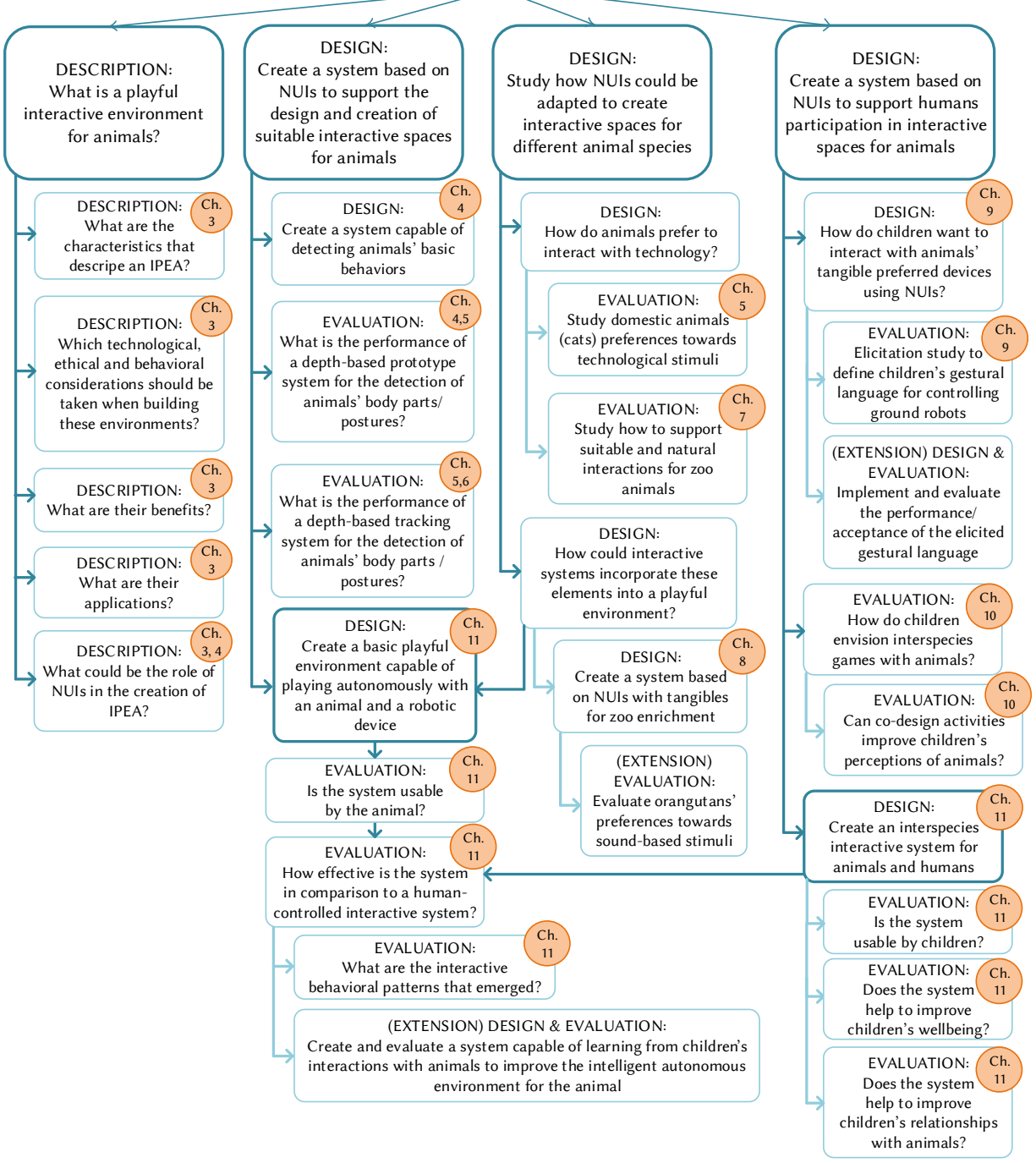

Figure 1.1: Problem decomposition (in rectangles) and the chapters in which the subjects are dealt with (in circles).

ful environment, as the system could move and adapt the robot to the animal's behavior in order to create an engaging playful activity. In zoos, non-technological everyday objects such as plastic balls were proposed as key elements in creating 
a playful NUI, which led to the design and development of a system capable of detecting such objects and augmenting the animals' spontaneous interactions with them by means of technological stimuli, e.g. by emitting sounds. With the aim of including humans as active participants in these interactive systems for animals, several participatory design studies were conducted with children. On one hand, an elicitation study was conducted in order to define a suitable gestural language for children to control a ground-based robot, which was the tangible interactive technology studied previously for domestic animals. On the other hand, a participatory design activity with children then aimed to discover how they envision interactive games with animals mediated by technology. The results of both exploratory studies, together with the knowledge derived from the proposed animal NUIs, were used to create and evaluate a basic playful environment capable of playing autonomously with an animal and a robotic device as well as allowing a human to play remotely with the animal.

The contributions of this thesis are manifold: first, the development of the first non-wearable depth-based tracking system to detect cats' postures and body parts and its evaluation; second, two designs for playful environments for two different animal species and contexts, i.e. domestic and zoo animals; third, a gestural language for the creation of NUIs based on children's preferred gestures for controlling ground-based minirobots; fourth, a promising co-design approach to improve children's perceptions of animals; fifth, the design, development and evaluation of an interspecies playful environment either for animals alone or for animals and humans based on NUIs with the potential to foster interspecies relationships; and, finally, a discussion of the future of interactive and intelligent technologies to support playful environments for animals based on the results of all the contributions proposed in this thesis, with the aim of informing future ACI research in this area.

\subsection{Outline of the Thesis}

In order to explore the aspects described in this chapter, this dissertation has been divided into four parts. The first part (including this chapter) frames the current literature in the ACI field, situating this work in its place within the field and describing the motivation for interactive and intelligent playful environments for animals. The second part describes the initial exploration of the use of Natural User Interfaces for animals and how systems are built capable of unobtrusively adapting to the animals' behavior. The third part explores the application of the proposed technological knowledge to create interactive spaces for a different animal species in a different context. The fourth part studies the co-design and development of Natural User Interfaces to include humans as participants within these interactive playful scenarios for animals. The final part of the dissertation includes a general discussion of the research conducted and future lines of research. A detailed description of the chapter structures is provided below: 
- Part I: Preliminaries. Besides the present chapter, Part I contains two additional chapters as an introduction to the field of Animal Computer Interaction and the current state of the art in terms of technological interventions, as well as a starting point to define the concept of interactive environments for animals. The works included in this part are the following:

- Chapter 2. This chapter contains the publication entitled Seven years after the Manifesto: Literature Review and Research Directions for Technologies in Animal Computer Interaction [119], which reviews the technological interventions found in the literature on Animal Computer Interaction since the formalization of the field back in 2011 with the ACI Manifesto [173]. This work provides an introduction to the field of ACI and its achievements over the last seven years in order to situate this thesis within the current research framework.

- Chapter 3 contains the book chapter entitled Envisioning Future Playful Interactive Environments for Animals [258], which analyzes the different approaches in which ACI studies have made use of technologically mediated playful interactions and communications. From this perspective, the chapter proposes a more autonomous approach to foster unrestricted animal interactions and behavior: Intelligent Playful Environments for Animals. The great potential of these systems is described, explaining how incorporating intelligent capabilities into playful environments could allow the system to learn from the animals' behavior and automatically adapt the game to their needs and preferences. Of all the proposed studies that could be carried out for the development of such systems, this thesis focuses on and contributes to the aspects described in Parts II, III and IV.

- Part II: Study and Recognition of Playful Interactions. This part focuses on the analysis of how animals prefer to interact with playful interactive devices and how the systems could recognize their interactions and types of behavior during the game in order to adapt the playful experience. The different chapters in this part are described as follows:

- Chapter 4 contains the paper entitled Developing a depth-based tracking system for interactive playful environments with animals [257], which explores the use of a Natural User Interface for animals based on nonwearable depth-based tracking to detect a cat's body posture and body parts in real time in a playful scenario. An initial prototype of the depth-based tracking system is described and evaluated, showing promising results in facilitating the recognition of animals' playful interactions with technology, while encouraging unrestricted spontaneous animal interactions in an open space. This system also opens the door to further 
exploration for its extension to other animal species and including human participants in the playful scenario.

- Chapter 5 contains the publication Towards Future Interactive Intelligent Systems for Animals: Study and Recognition of Embodied Interactions [260]. Digital playful activities which include animals as participants should attract and maintain their attention by using suitable technological artifacts and adapting the activity to the animals' preferences. To achieve this, the first step is to find the best technological artifacts for a specific animal species in terms of engagement and interest and to study how the animals best interact with them. This chapter describes a pioneering observational study conducted on cats with the aim of exploring their interactions and preferences for different technological artifacts. A discussion of the proposed technologies and the factors that have been observed to affect cats' preferences and behaviors is reported to assist in the design and development of suitable and engaging interactive environments for them. An extension of the depth-based tracking system proposed in Chapter 4 [257] is also presented and evaluated, providing an initial comparison of the knowledge-based learning approach used in [257] with a supervised learning approach using two well-known machine learning classifiers.

- Chapter 6 contains the paper entitled Assessing machine learning classifiers for the detection of animals' behavior using depth-based tracking [255]. This paper provides an extensive in-depth description and evaluation of the non-wearable tracking system for automatic detection of cats' postures and body parts, as well as an exhaustive evaluation of the performance of several classification algorithms based on both a supervised and a knowledge-based approach. The evaluation of both approaches of the proposed system shows promise for advancing research on animal behavior recognition both in and outside the field of Animal Computer Interaction, and this system could be further extended to other animal species.

- Part III: Tangible User Interfaces for Animal Enrichment. As animals of different species behave differently and also have different physiological characteristics, the ways in which a system extracts information from their behavior would be different, and so would be the design of an artifact capable of providing this contextual information to the system. Animals' playful or exploratory behavior of a system also differs from one species to another. For these reasons, this thesis explores how NUIs based on body tracking could be designed and used for different species in different environmental contexts. This part aims to illustrate this design process to validate the flexibility of the approach and is comprised of the following chapters: 
- Chapter 7 contains the work Tangible User Interfaces for Zoo Enrichment [252]. In this paper an interactive system for orangutans in a zoo environment is described and discussed. In this environment the range of elements the animals can interact with is limited in order to prioritize their safety. In this regard, as orangutans are much stronger than humans, this makes it extremely difficult to provide them with free access to technology inside their enclosure. As a result, the provided technology might not be as compelling and engaging as it could be, and the interaction may be less natural and limited to when the zoo keepers are present. Hence, aligned with the goals and methodology of the Kinecting with Orangutans project [343], this work proposes the use of depth-based sensors to track the orangutans' movements of everyday non-technological objects. These interactions could then be digitally augmented with appropriate technological stimuli, such as sounds or visual projections, to create varied and adaptable enrichment activities, e.g. produce different sounds based on movement or make a visual projection react to the orangutans' movements.

- Chapter 8 contains the publication Sound to your Objects: A Novel Design Approach to Evaluate Orangutans' Interest in Sound-based Stimuli [247]. This paper is based on the proposed design of tangible interfaces for orangutans presented in Chapter 7 [252] and describes its implementation using sound-based enrichment to augment orangutans' tracked movements of tangible objects. This paper explains the decision and design process conducted jointly by keepers and researchers, which highlighted the orangutans' curiosity in sound-based stimuli. The implemented system was aimed to study the types of behavior and gestures orangutans would spontaneously use to interact with the system, and to evaluate the orangutans' preference for different auditory stimuli. However, the orangutans' unexpected interactions and reactions showed that the system should be further adapted in order to evaluate such goals.

- Part IV: Interspecies Participatory Design of Playful Interactive Environments. Human participation in playful environments for animals could help to improve not only the wellbeing of animals and humans, but also their interrelationships and bonds by creating a shared space for their amusement. In this regard, it is essential that future generations develop empathy and create strong bonds with the animals in our ecosystems to work towards a more inclusive world for all species. This part of the thesis thus explores how humans, and especially children, envision their participation in such interactive environments, how NUIs could support these interactions, and how interspecies playful interactive environments can help to improve the development of intelligent and adaptive systems for animals while helping 
children's wellbeing and relationships with them. The works included in this section are as follows:

- Chapter 9 contains the paper Interactive spaces for children: gesture elicitation for controlling ground mini-robots [251]. Based on the idea of providing animals with tangible interactive elements which they can explore within the interactive space, this study focuses on the elicitation of children's preferred gestures for controlling tangible robots. The goal of the study was to propose a NUI based on gestures and body language so that children could easily manipulate robots within an interactive space for animals, allowing them to focus on the animal participant and its interactions while moving freely around the space. The main outcome of the study is the definition of a gestural language based on body interactions suitable for children aged 6-12 years old.

- Chapter 10 contains the work entitled Designing interspecies playful interactions: studying children perceptions of games with animals [248]. This paper proposes a co-design activity with children in which they could draw and describe how they would like to play with animals of different species. Two different settings were proposed, one in which the activity was mediated by a technological device selected by the children themselves and another with no technological elements involved. This comparison, along with the pre and post questionnaires, showed an improved positive perception of the animals after the design activity, while the children were also observed to reflect on their designs, raising their awareness of the need for more animal-centric games.

- Chapter 11 contains the publication Remote Interspecies Interactions: Improving humans and animals' wellbeing through mobile playful spaces [246], which describes the design and development of an interspecies playful environment for animals and humans that builds upon the insights obtained from the different studies described in this manuscript. Due to time limitations, considering the effort that the development of an accurate and reliable tracking system as the one proposed in 9 [251] would require, and observing children's designs for playful experiences with animals in 10 [248], the children's interactions within this system would be by means of a mobile application for screen-based devices, which was the most common technological device selected by children in 9 [251]. This application allows children to control a tangible robot in order to play remotely with a dog located in a canine daycare facility. An autonomous interactive system with basic intelligent features for animal play is also described and evaluated. This work compares the two different approaches in terms of their suitability, usability and 
human/animal engagement, identifying behavioral patterns and future promising improvements.

- Part V: Closure. This last part of the thesis provides a discussion of the contributions and aspects tackled in this manuscript, as well as future recommendations to build upon the developed technologies and designed systems in order to contribute to the advance of research in ACI.

To sum up, the work described in this thesis has produced several research papers, some of which are included in this dissertation, while others have been excluded as they were aimed at more specific venues. In particular, this dissertation comprises 10 research papers: 5 read at conferences $\left(1 \mathrm{CORE} \mathrm{A}^{*}\right.$ workshop, 1 CORE A, 1 CORE B, 2 ACI conference), 2 published in scientific journals (1 JCR Q1, 1 OA journal with $30 \%$ contribution as second author), 1 book chapter, and 2 JCR journals which are still under review. In addition, 6 research papers derived from this thesis have also been produced, but were not included in this manuscript (1 CORE A* poster [253], 1 CORE A* workshop [249], 2 Doctoral Consortium [250, 259], 1 ACI workshop [256], 1 ACI conference [254]). 


\section{Chapter 2}

\section{Seven years after the}

Manifesto: Literature Review and Research Directions for Technologies in Animal Computer Interaction

Ilyena Hirskyj-Douglas ${ }^{1}$, Patricia Pons ${ }^{2}$, Janet C Read ${ }^{3}$, Javier Jaen ${ }^{2}$

${ }^{1}$ Department of Computer Science, Aalto University, Helsinki, Finland

${ }^{2}$ ISSI Group, Departamento de Sistemas Informaticos y Computacion,

Universitat Politècnica de València, Valencia, Spain

\footnotetext{
${ }^{3}$ Department of Computer Science,

University of Central Lancashire, Preston, England
}

Published in Multimodal Technologies and Interaction 30, 2, (2018). doi.org/10.3390/mti2020030 


\section{Abstract}

As technologies diversify and become embedded in everyday lives, the technologies we expose to animals, and the new technologies being developed for animals within the field of Animal Computer Interaction (ACI) are increasing. As we approach seven years since the ACI manifesto, which grounded the field within Human Computer Interaction and Computer Science, this thematic literature review looks at the technologies developed for (non-human) animals. Technologies that are analysed include tangible and physical, haptic and wearable, olfactory, screen technology and tracking systems. The conversation explores what exactly $\mathrm{ACI}$ is whilst questioning what it means to be animal by considering the impact and loop between machine and animal interactivity. The findings of this review are expected to form the first grounding foundation of ACI technologies informing future research in animal computing as well as suggesting future areas for exploration.

\section{$2.1 \quad$ Introduction}

The well-being, behaviours, and physical characteristics of animals have long been studied within animal biology sciences but the landscape changes as the understanding of animals evolves. In the late twentieth century, studies were conducted into the ways that some animals behave in human-animal situations and subsequently, these studies have moved towards the ability of animals to assist humans and thus improve the human condition [54]. As technology has become embedded in the human condition, it has also become of interest in terms of how it affects the human-animal relation aiming to move away from anthropocentric work towards an animal-centric focus. Technology today has been shown to be useful for playful interactions between humans and animals $[258,351]$, for monitoring animals [176, 273], training animals [204] and supporting animals that care for humans [282, 369]. This has driven researchers, for societal and economic reasons, to explore animals within technological situations.

One of the main initial aims for the study of Animal Computer Interaction (ACI) has been "to understand the interaction between animals and computing technology within the contexts in which animals habitually live, are active, and socialize with members of the same or other species, including humans" [173] . As a relatively new field, being coined in 2011 in the ACI Manifesto [173] , it has taken its main reference from Human Computer Interaction (HCI) [177, 273, 351], which in turn has led to an early focus on studies of the usability of technology and the user experience of animals to influence the design of interactive solutions $[164,316]$. Frameworks have been constructed for ACI technology in the areas of interaction design [316], Human Computer Interaction (HCI) [351], ubiquitous computing [176] and game design [363]. Some of these frameworks aim to reveal 
the role of technology within a human-animal interaction $[176,351]$, whilst others aim to minimise the human role to more fully design for the animals' unique needs. Whilst motivation for animal-computer technologies is often welfare based [176], ACI also attends to other aspects, including the pet entertainment and holistic well-being sectors where many commercially available products exist [47, 72]. The terminology of welfare we use within this work is not only in reference towards the animal being healthy, nourished, safe, able to express innate behaviour, comfortable and not suffering from any negative states (as defined by medical agencies) but also in viewing the animal as a 'whole' [348]. Within ACI, welfare is inherently linked towards animal centeredness by researchers who allow consent through walking away behaviour (innate behaviour), research into how to make systems more suitable for animals (comfortable), and often seeking ways to monitor health (healthy and nourished).

Academic studies pertinent to the design of ACI technologies have increased in number over the last seven years since the publication of the ACI Manifesto [173], the introduction of the ACISIG at the CHI conference [178], the first, second, third, fourth and the coming-soon fifth International Animal-Computer Interaction Conferences $(2014,2015,2016,2017,2018)$, and workshops at major HCI conferences (ISAWEL'14, ACI@BHCI 2015, NordiCHI 2014 and 2016 and OzACI@OzCHI 2017). As interest has grown in this field the workshops and events have become more specialised with: ACI@Measuring Behavior 2016, HCI goes to the zoo at CHI'16, Research Methods for ACI, ZooJam at ACI'16, and Technology for Bonding in Human-Animal Interaction and FarmJam at ACI'17.

However, as we embark on the seventh year since the ACI Manifesto [173] there has yet to be an in-depth literature review delineating from the foundations of ACI, towards the creation and use of technical means and their interrelation with animals, looking forwards towards potential areas for future research. Whilst literature reviews around the field of ACI exist, such as the one for smart computing and sensing technologies for animal welfare [143], there has yet to be a direct overview of technologies within ACI. This chronicle begins by briefly exploring what ACI is and considering how the fields of animal behaviour and HCI intersect and contribute towards the embodied work. What an interaction is, or can be, defined as is questioned in this narrative. A thematic analysis of technologies within ACI is then delivered to investigate how the current body of research adds to the current overall field narratives. Drawing from this, a discussion is held on potential technological areas that ACI has yet to address, identifying questions opened through this review and concluding in an overall summary of the field.

This technology driven thematic literature review is intended to both bring clarity to those entering the field whilst highlighting potential areas of interest for those currently working in the field. Whilst this review does not tackle ethical, methodological, legal, economic and philosophical issues surrounding ACI, 
it is hoped that those embroiled in such topics may find this narrative useful in initiating discussions.

\subsection{What is ACI?}

The natural question that opens the literature review, and is our starting point, is 'What is ACI?' At first glance, ACI can be defined by its components: the animal, the computer, and the way they work together (as HCI is defined [141]). It can also be defined, as identified within its early work, by the main goal that it seeks to meet, that being: 'usability through a discussion about factors involved such as constraints, functionally and the user' $[175,181]$.

In seeking to differentiate ACI from HCI, however, it is important to step back and consider what we define as an animal. The Cambridge Dictionary (2016) offers two definitions:

1. Something that lives and moves but is not a human, bird, fish, or insect.

2. Anything that lives and moves, including people, birds, etc.

These definitions expose contrasting views and show two ways of looking at ACI: either as (1) an offshoot of computer science into non-human animals or (2) the encircling of HCI, CCI (Child Computer Interaction) and other subfields, into an overall look at all animals, including humans as animals. Whilst the debate over the distinction between human and non-human animals has far-reaching roots back to Darwin's approach in the Origin of Species [52], it is probably fair to say that it is largely about humans' unique abilities, and beliefs, about the uniqueness of species. This latter point has been interpreted differently over time according to the mood of the day and the understanding of mind and action. Biologically speaking, the definition of animal refers to all members of the kingdom Animalia ( The American Heritage Dictionary) but colloquial use of animal frequently refers to non-human animals in an umbrella terminology. The tension between the two positions challenges ACI to consider methodologically the position that animals hold within the research space. Tattersall describes well the problem space writing that 'We have similarities with everything else in nature; it would be astonishing if we didn't. But we've got to look at the differences' [121]. From here on in, this narrative will refer to ACI as assuming the exclusion of the human for clarity but will descend from the vantage point of anti-dichotomy within the animal hierarchy by focusing on, like Tattersall, the differences between species' use of computers and computer technology.

Reflecting on the human-animal difference, the field of ACI emerged in computer science research via HCI but technology had previously been used to explore animal behaviour in other research fields (e.g. bio-logging within animal ecology and 
technological interventions for animal cognition studies). The inclusion of the term 'computer' in ACI assumes that the technology with which the animal is interacting, or which is facilitating some behaviour, is embedded with computer technology and so is able to react and interact with elements in the environment.

\subsubsection{What is Interaction?}

Interaction, and its study, is elemental to HCI and thus also to ACI. In ACI this interaction always includes the animal and the technology but often will also include a human owner, researcher and/or carer. The study of ACI aims to enhance interaction by developing methods, philosophical stances and theories within this space. However, the terminology of what it means to have an interactive system has not yet been clearly defined within ACI. Interaction can be seen in a broad way as the framing of the relationship between people and the objects designed for them [35] but in ACI, as in HCI, interaction is more often seen as an archetypal structure, such as the feedback loop [60] where reference is made to 'an interaction', which is the communication between system and user. This maps onto what is described in animal behaviourism as stimuli and responses [96]. Within this definition, interaction in ACI refers to the way that the animal reacts to the technology and in return the way that the technology then responds to the animal within the feedback loop. This typical definition has been questioned by Aspling \& Juhlin [11] who instead refer to interaction as a dyadic, direct and strategic interaction between multiple agencies arguing instead for Actor-Network Theory and Goffman's [94] notion of strategic interaction in ACI.

The term 'interaction' is used throughout this literature review, but it is acknowledged, in the sense of Buchanan's [35] definition of interaction, that the degree to which an animal can meaningfully interact with a computer system is unknown as animals' intentions, and what animals perceive as possible to do within a computer system, are unidentified. This is not to imply that animals cannot have implicit or unaware interactions that are meaningful, but that the scope behind terming what is meaningful to an animal within the interaction is unknown. In HCI, this degree of representation is described in the theoretical framework coined by Norman [218] as 'the gulf of execution'. A model of this within ACI is shown in Figure 2.1. Research endeavour in ACI explores this 'gulf' of the animal user's intentions $[89,118,175]$ and considers if this can be represented, directly perceived and interpreted [369]. This is explored through trying to capture the animal user's actions and intentions. In human-human and human-computer communication, there is a rich two-way feedback loop of interactivity where there is derived meaning gathered from the actions taken, the interpretation, and in return the output delivered. There also appears evidence of this feedback loop in animal-human communication between animal and trainer, such as when they interact. 


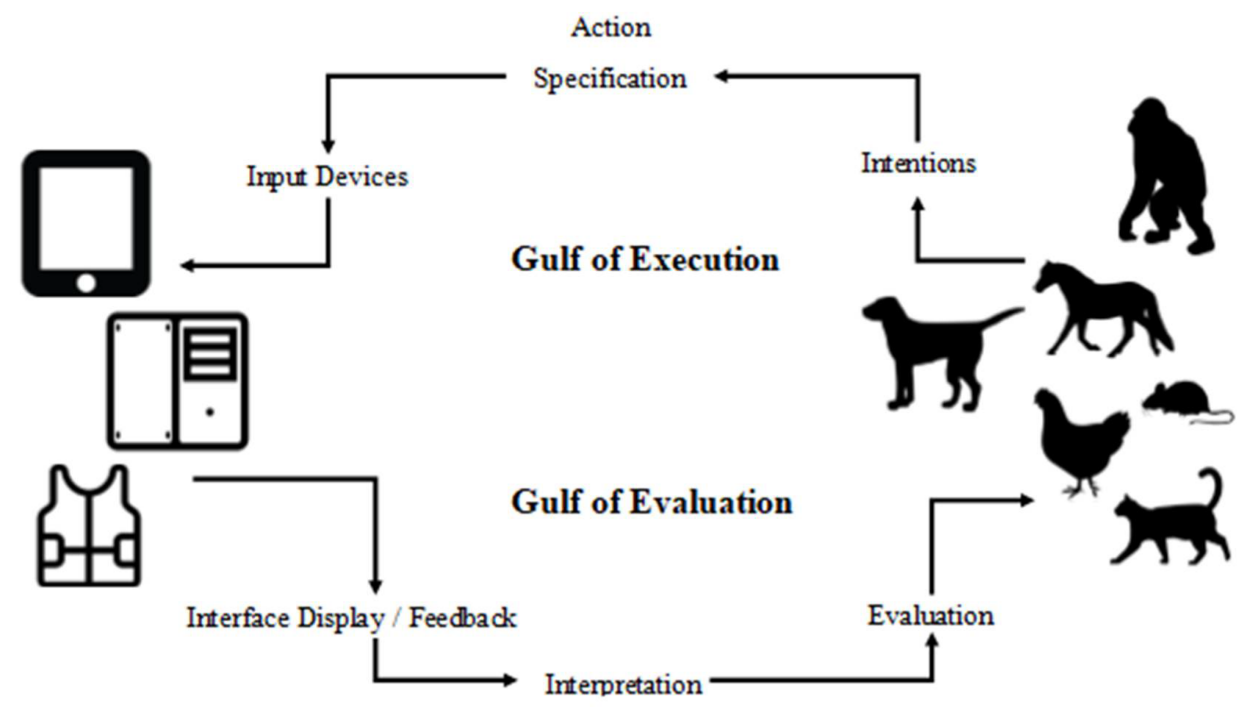

Figure 2.1: Representation of the gulf of execution in ACI systems.

It is in this way that often ACI explores the gulf of execution, the top half of the feedback loop, and it is acknowledged that the loop may not be fully closed. In ACI this is due to the bottom half of the loop (gulf of evaluation) being unknown, that is, an animal interaction can be captured, but what meaning this has to the animal (interpretation) is yet to be discovered. For these reasons, ACI is not primarily about designing complete interactive systems but more about exploring elements within them. These elements include 'looking at behaviours' and 'attending to behaviours', but not the reasoning behind animals' actions (intentions). Within this space, all we can do as researchers is interpret these behaviours. This same interpretation is very often the case in Child Computer Interaction (CCI) and sometimes also in adult studies in those cases where it can be hard to draw at the intention behind the interaction loop. As ACI is a relatively new field, there is clearly more research to be undertaken to explore these gulfs, particularly with support from the animal psychological and behavioural fields.

The feedback loop presented in Figure 2.1 has also been modelled by Freil et al. [74], who also extended from Norman's [218] gulfs towards a dog computing training scenario. Unlike as described here, Freil et al. [74] considered the model to be fully closed. Our view, in this paper, is that for the gulf of evaluation to have execution errors such as slips and mistakes, this requires specific knowledge of the animal's intentions. This literature review therefore considers the animal's intentions (as termed by Freil et al. [74]) to be human interpretations (as noted within the gulf of evaluation above) of the animal's behaviour. 
Drawing back to the published literature from ACI, interaction has been considered here in a broad sense including the animal controlling a system [33, 276, 282, 369], systems detecting an animal's behaviour [171, 330], systems reacting to an animal's behaviour $[89,180]$ and the animal interacting with the human through its behaviour $[164,165,193,204,273,323]$. As noted within the ACI manifesto [173], the interaction can also be explored by improving the usability of systems as well as by creating a meaningful experience for the animal. This is evidenced in work that has studied how animals can input to technology [137, 240, $280,369]$, how animals can be soothed or stimulated by technology $[17,75,89]$ and how animals and humans can be connected through technology [41, 165, 193, 204, 273]. An interesting point within these intersections is the transferal of these technologies across species and across disciplines, that will also be addressed in this manuscript through modelling the technological system's space within ACI.

\subsection{Technologies in ACI}

This section reviews the ACI literature in terms of the different technological approaches present in ACI related works. The following subsections describe these innovations in further detail: tangible and physical objects, haptic and wearable technologies, olfactory interfaces, screen interfaces and tracking mechanisms. This classification has been originated into subgroups via the thematic analysis of the technology interface in order to explore those areas in further depth. Each section will summarize the current narrative of the technology interface and will then bring these findings into an overall model of technologies in ACI. This narrative aims to provide a general technological picture of the field, with future trends and opportunities discussed later on in Section 2.4.

\subsubsection{Model of Technologies within ACI}

Figure 2.2 illustrates the framework, proposed by this literature review, building from Jukan et al's. [143] model regarding technologies for animal welfare. The framework proposed here focuses more towards overall technologies in ACI and thus expands Jukan et al's work. Reviewed here are: Tangible \& Physical technologies, Haptic \& Wearable technologies, Olfactory interfaces, Screen-based interfaces and Tracking technologies. These various technologies seek to aid animals in various instances; such as to aid human health, control the animal, enable data exchange, assist working animals, aid service animals, enrich play and monitor the animal.

This review, as suggested within Mancini's early work [174], will include technologies directly involved within animal computing and not only technologies informed by animal computer interaction. This appreciates the difference between a technology user and an animal within the technology scenario. Lawson et al. 


\begin{tabular}{|c|c|c|c|c|}
\hline Tangible \& Physical & Haptic \& Wearable & Olfactory & Screen Technology & Tracking Technologies \\
\hline Control & Control & Control & Enrichment & Enrichment \\
\hline Communication & Communication & Communication & Human Health & Monitoring \\
\hline Enrichment & Service Animal & \multirow{4}{*}{$\begin{array}{c}\text { Working } \\
\text { Animal }\end{array}$} & Playful & Playful \\
\hline Human Health & \multirow{3}{*}{$\begin{array}{c}\text { Working } \\
\text { Animal }\end{array}$} & & Service Animal & \\
\hline Playful & & & Working & \\
\hline Welfare & & & Animal & \\
\hline
\end{tabular}

Figure 2.2: Framework for technologies in ACI (building from Jukan et al. [143]).

[160] tackled this issue as it refers to consent by drawing from the ACI Manifesto goals and paraphrasing Eric Baumer's terminology of "usees", being those situations where technologies are imposed upon animals without consideration, making them inherent users and participants submitting their data. Whilst it is beyond the scope of this work to define the terminology of a user, it is noted that not all animals that are directly involved in technologies are active users - some may instead be a wearer $[171,232,234]$ or may inform a system $[257,360]$ rather than being an active user inputting information [280, 369] or directly controlling a system [114].

Following this classification presented above in Figure 2.2, Table 2.1 presents a list of the works considered within this literature review.

\subsubsection{Tangible and Physical Objects}

As technologies began becoming intertwined with animals, these early systems primarily focused around the animal-human communication paradigm where humans sought to communicate with animals. These systems included the LANA (LANguage Analogue) project where chimpanzees used "lexigrams" to create sentences and communicate with humans [285] and early button systems to allow dolphins to ask for certain toys and food [272]. Whilst early research focused around the cognitive abilities of animals, Resner [273] challenged this by looking at the animaltechnology relationship in more of a HCI stance focusing on Interaction Design $(\mathrm{IxD}) . \mathrm{Hu}$ et al. [129] took this idea further by creating a web-based system to allow humans to remotely interact with their dogs by giving them treats, talking to them through speakers or throwing a tennis ball they could catch, The system aimed to improve pet-human interaction and came from a HCI IxD standpoint. 







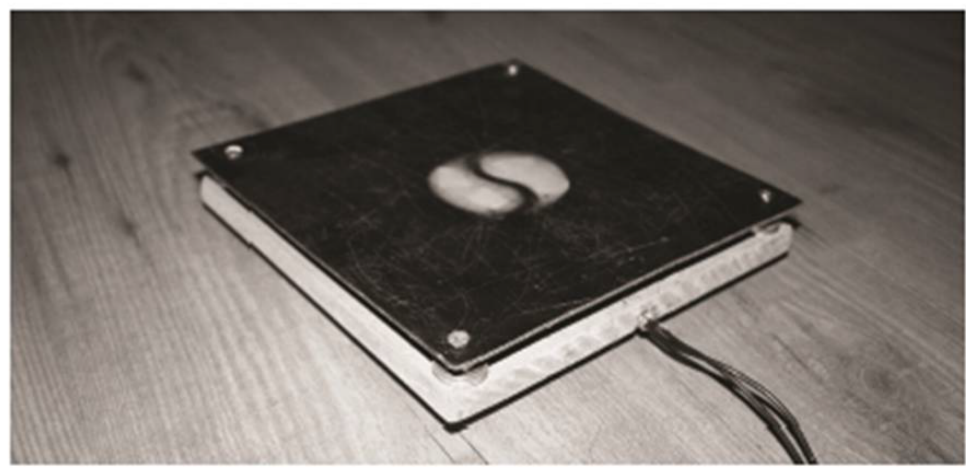

Figure 2.3: Button system used as a pressure plate to dispense treats [89].

These early archetypes were primarily based on tangible and physical objects and collectively contribute a large number of ACI technology interfaces.

In ACI, exploration has been conducted with tangible and physical objects such as pulling devices [280, 281], buttons [89, 181] (Figure 2.3), digitally augmented toys such as tree trunks and pulleys [76, 77] and plastic balls [247], and with robots $[87,88]$.

Robinson et al. [280] created a pulley system for a medical assistant dog to call for help using a tug toy as an interaction mechanism, as this is a familiar way of interaction for a dog. Following an iterative user-centred design process, in which they tested several materials and configurations, they developed a high-fidelity prototype of a dog alarm system that could work for several scenarios [281].

Tangible objects have also been explored within ACI in their ability to give animals control over their environment and any proposed technological interventions. Along this line of thought, French et al. [75] used ordinary items found within an elephant's enclosure to allow the automatic use of devices - in this case a shower. With orangutans, Pons et al. [247] studied how non-technological everyday objects could be augmented with auditory digital responses in order to provide a novel form of enrichment for these animals, building on their intrinsic dexterity with object manipulation. More recently, Gupfinger and Kaltenbrunner [103] have explored the use of tangible technological mediators with grey parrots so that they can produce sounds and music by activating a joystick or a rope swing; this builds from Ritvo \& Allison's [276] work with orangutans and music (see Section 2.3.5). A similar approach was followed to develop a tangible cylinder for an orangutan's enclosure that produces sounds when rotated [111]. The cylinder, in this case, was attached to the wall and it had a maze-puzzle embedded in it. Orangutans explored the cylinder freely, by rotating or touching it, which produced sounds 


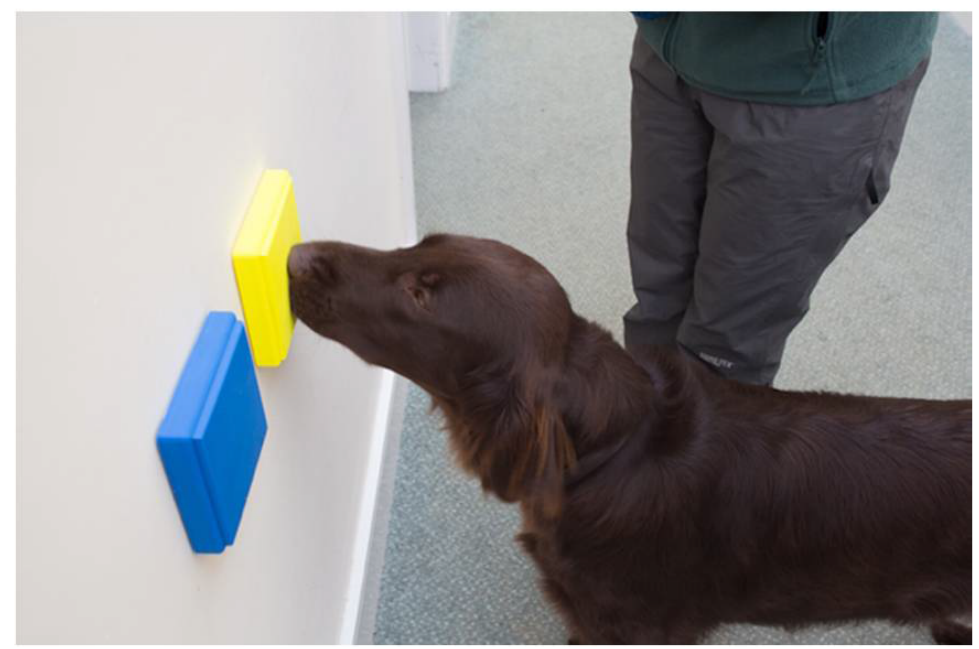

Figure 2.4: A dog activating a switch [181]. Photo courtesy of The Open University.

not only in their enclosure but also in the human visitors' side, augmenting and enriching the human experiences of viewing captive animals at a zoo. Technology has opened a whole new range of possibilities in terms of animal enrichment, as everyday objects can now be enhanced with sensors to create more varied scenarios. For example, researchers and staff at San Francisco Zoo have created a giant puzzle feeder for rhinos [156]. The system dispenses treats when the animals investigate and manipulate it, fostering their natural foraging behaviours [156].

Button-like devices have been one of the most common approaches when working with dogs, allowing these animals to intuitively interact with either their paws or noses. Geurtsen et al. [89] used used a pressure plate button to give dogs treats - a method in line with current consumer products for dogs such as CleverPet [47]. Another investigation of button systems with service dogs was by Mancini et al. [181] who sought to look at extending dog accessibility towards their environment in mobility dogs by mapping out the challenges faced in a human domain (Figure 2.4).

Gergely et al. [87] examined interaction with Unidentified Moving Objects (UMO) by investigating how dogs act socially with robots. Gergely et al. [87] found that dogs act socially towards UMOs from expectations of the system over a short period, offering up an indication towards social robots for dogs. Previous to this work, Singh \& Yong [303] used robotic tails on robots which could move positions (wag, raise, lower and hold straight) to investigate dog tail communication states; this work is yet to be tested with other dogs to draw conclusive 
results. Westerlaken et al. [349] used a design observational study with dogs and robots, applying different variations to the robotic devices, such as puzzle or treatdispenser cases, and with tangible objects that produced sound or smell. They observed that the material of the robot conditioned the interest of the dogs in the game: when the dogs realized they could not grab the robotic ball with their mouths, they began to lose interest in the game. One of their main observations was that different traits in each individual dog could lead to different kinds of playful preferences. In a similar fashion, Pons et al. [260] conducted an observational study with seven cats and two different small robots to methodologically investigate cats' interactions and to see which devices the cats preferred. The authors found that the age and size of the cats were a factor, as cats usually preferred to interact with smaller robots in order to replicate a playful hunting behaviour with them. This aligns with Westerlaken et al.'s [349] proposition of animals' personal traits playing an important role within the interactions.

Byrne et al. [38] have recently proposed a technological approach to predict the suitability of dogs for assistive dog training programs. The authors have explored whether aspects of canine temperament can be detected from dogs' interactions with sensors embedded in two instrumented dog toys: a silicone ball and a silicone tug sensor. From the dog's natural interactions with the instrumented devices, a prediction model has been created that allows for the assessment of the dogs' outcomes in the program with an $87.5 \%$ average accuracy.

Whilst tangible and physical objects have a long history within animal technologies, the way in which these types of interfaces have been investigated has developed from a purely cognitive and behavioural standpoint towards a more interactive paradigm. Within this area the largest body of work conducted has been for service and working animals, with recent moves towards the zoo and pet fields. There are clear gaps present within the research to investigate not only new technologies, but also to investigate how animals respond to, and can be trained more efficiently towards, technological systems that they hold, sniff, point, paw, tug and use in both regards towards the interface and interaction model. As the ACI field advances, work is currently shifting towards a non-training approach, especially in the context of enrichment, play or technologies aiming towards giving the animal control. A more animal-centric design perspective in these scenarios has been to deploy the technology and let the animal "become with" $[107,361]$, where no interaction is wrong, so as to iteratively re-design the technology based on the observed interactions in a research through design fashion [76]. More research has yet to be done in terms of animal-centred design methodologies to account for these new insights as the field knowledge grows, questioning which user (man or animal) is really in the centre of the design [116]. 


\subsubsection{Haptic and Wearable Technologies}

Haptic technology is defined as "the science of applying touch sensation and control to interact with computer developed applications" [310]. Therefore, a haptic device allows the user interface to be the animal's body sensations; this includes especially tactile feedback to perform actions and receive input. One method of instantiating a haptic interface is the use of vibrotactile technologies which can range from skin surface monitoring to vibrating interfaces. Lee et al.'s [164] work, one of the first ACI contributions, used a haptic wearable jacket on chickens in order to allow a human user to remotely stroke the chicken. A similar approach was presented by Réhman \& Li [270], who proposed remote communication between humans and animals via vibrotactile feedback for the animal. More recently, haptic vibrotactile interfaces have been implemented for dogs, using the same research method as Lee et al. [164]. Britt et al. [33] trained a dog using a vibrotactile haptic vest that allowed a human handler to remotely guide the dog using vocal commands as well as applying vibrations on the vest. This idea has also been explored by Byrne et al. [37] using haptic cues to assist in training, an approach also evident in Morrison et al. [204] who iteratively designed a wearable vibrotactile vest to assist in direction pointing for hunting dogs, arguing that vibrotactile input aids the collaborative discussion within hunting between dog and owner. These vibrotactile haptic interfaces, however, have so far only been used with dogs and chickens, where these devices have been proposed to have a positive reaction from animals [164, 165] and successful training in reported behaviours [33, 37, 204], leaving this User Interface (UI) open for future exploration.

Vibrotactiles however, are not constrained to animals using wearable haptics. French et al. [77] developed vibrotactile buttons made of different materials for elephants. These interactive devices were aimed towards triggering elephants' curiosity to explore the device based on the haptic feedback they received when approaching the material with their trunks. The vibrotactile buttons allowed elephants to produce different sounds in their enclosure. In one extreme case, vibrotactile feedback was used with insects (crickets) by applying vibrations to the ground on which the crickets stood [61]; although the authors did face problems with animal shedding in reaction to vibrations. A follow up study investigated if playing PAC-MAN against an Artificial Intelligence (AI) is perceived as 'funnier' than playing against a system replicating animals' movements [62].

Wearable computing has been defined as "the study or practice of inventing, designing, building, or using miniature body-borne computational and sensory devices" [183]. Wearables in HCI have been used for a wide variety of purposes, and their proliferation has also reached the field of ACI. For instance, biotelemetry devices can be considered as wearable interfaces although there remains an argument on the term 'user' being applied to this scenario, being preferred the term 'wearer'. Biotelemetry devices have been used for many years in biological research, playing an important role in the development of behavioural science and in ACI research 
which looks at the wearability of these devices [232, 233]. Biotelemetry devices have also been used to inform blind dog owners of real-time heartbeat and respiration rates of their dogs while taking a walk [193]. The information provided by the biotelemetry sensors on the dog was transmitted to the human handler by means of either vibrotactile feedback or audio devices. Mealin et al. [193] found that although vibrotactile interfaces provide more accurate responses, dog handlers preferred to use audio devices. In a more recent work Mealin et al. [192] designed a system that collected physiological data from guide dogs in training using wearable devices. The collected data facilitated objective analysis of the dog during early stages of training, helping to predict how successful a dog would be in the program.

Several works have addressed the necessity of tracking animals, beyond biotelemetry devices, in different scenarios, this is expanded on in Section 2.3.6. However, despite the increase in tracking technologies, the most common method to gather information about the animal has been to use wearable harnesses or collars with attached technological devices providing information to the system in charge of processing the information. One of the most basic methods for animal tracking in outdoor scenarios has relied on GPS or radio-frequency localization, attaching the emitter devices to a collar or harness. These systems only give information on the animal's location and have been used by pet owners, mostly to assess their dogs' locations and to determine whether or not they are in trouble [179]; this technology also exists in commercial products such as FitBark [72]. This technology has also been used during hunting activities with dogs, allowing the human leading the hunting activity to interpret the movements of the dog in the field by following its signal on a handheld display [235, 344].

Whilst GPS is useful, several outdoor scenarios require more precise information about the animals' movements or body postures during the activity, and even some kind of communication from the animal to the human side. As an example, determining the pose of the animal is of vital importance in the case of Search and Rescue (SAR) dogs which often have to work away from human sight; it would be beneficial if the handlers knew the dogs' location and pose. This type of recognition of animals' postures and activities is usually performed using accelerometers, gyroscopes or other inertial measurements. Most of the works in ACI using these products have been with dogs, probably because of their use as working and assistive animals [30, 371]. Most of the works based on wearable devices for activity/posture recognition are based on the use of a tri-axial accelerometer located at the dog's collar, these then apply classification techniques to the data obtained from the accelerometer in order to recognize the activity/posture. There are several devices for dogs, some of them even commercial, such as Whistle ${ }^{\circledR}$ [354], FitBark ${ }^{\circledR}[72]$ or WagTag $^{\mathrm{TM}}$ [346] which make use of a tri-axial accelerometer to perform basic activity level recognition. In [158], dogs wear a tri-axial accelerometer on the collar and, after being trained with a k-nearest neighbours 
$(\mathrm{kNN})$ classifier, the system is able to differentiate between 14 activities and 2 postures.

Within the FIDO project [133], researchers have been studying how wearable devices could mediate the communication between working dogs and their handlers, much like Morrison et al. [204]. The FIDO project has undertaken extensive work on providing dogs with suitable wearable activators [132]. In addition, they have also considered to mediate this communication by recognizing motion-based dog gestures - sit, spin, roll, jump, etc. - using a three-axis accelerometer attached to the front of a service dog harness [328]. More recently, they have studied the use of a dog collar with an accelerometer and gyroscope for the recognition of head gestures in dogs $[329,330]$. Whilst they struggled with the sensitivity of such devices, the researchers did find that gesture recognition through collars was viable and they pointed to looking to how a dog was trained (i.e., with a leash) to give an indication of gestures that could be instantiated in such systems.

The effectiveness of wearable harnesses with several inertial measurement units located along the harness has also been studied. The work of Ribeiro et al. [274] uses the angles of two accelerometers on different locations on the dogs' harness to develop an algorithm capable of estimating four poses including: standing, lying down, sitting, and walking. Another project [30,34] extended this idea by using more inertial measurement units located on the optimal locations of a dog's body, which have been determined attending to the algorithm's performance and the dog's comfort and physionomy. Using the information provided by these units and applying machine learning techniques, five static postures and three dynamic behaviours can be identified. They have also compared the performance of the classification algorithm using supervised against unsupervised classification methods [360]. The knowledge from these previous studies came together in Majikes et al. [171] research, which used a harness vest system with dogs, like those used by Byrne et al. [37], Britt [33] and Lemasson et al. [165], to monitor a dog's posture during eating, standing, lying, sitting and standing on two legs (Figure 2.5).

In the vest system for dogs, Majikes et al. [171] extended the usefulness of haptic devices by mixing the vest outputs with human analysis for interpretation and found that this can lead to a higher rate of success in training. The authors in the future hope to take this device into a fully autonomous system and have cue trained behaviours leading to complex behaviours.

Acceleration data-loggers are also a common and efficient way of detecting cats' body postures and frequent behaviours based on movement [340]. Commercial devices for cat activity recognition are also available, such as PawTrack ${ }^{\circledR}$ [238], which detects whether the cat is at home or outside, and offers GPS geolocation for outdoor walks. However, it does not monitor any activity nor gesture such as the non-commercial research Cat@Log [367] device. Cat@Log consists of a cat collar device with several sensors: a camera, a GPS, an accelerometer, a Bluetooth 


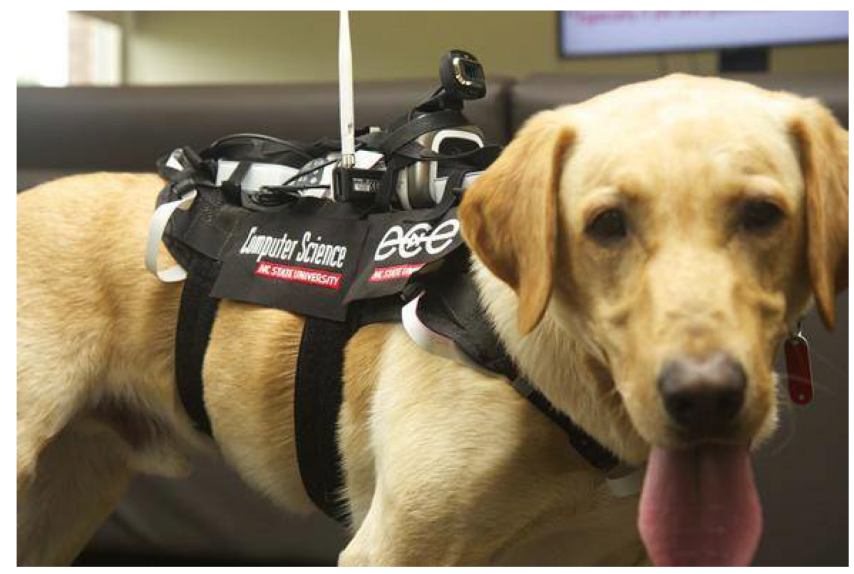

Figure 2.5: Posture system used by Majikes et al. [171].

module, a battery and a micro SD card. The camera provides videos of the cat's view, while the accelerometer data allows activity recognition such as sleeping, jumping, walking or scratching.

Canine Amusement and Training [359] present a home based wearable tracking system for dogs away from accelerometers. This consists of Infra-Red (IR) emitters attached to the dog's harness, and a Wiimote's IR camera placed on the ceiling. The system detects the location and posture of the animal by tracking the IR emissions of the harness using the Wiimote. The detected postures and location are used by the system to determine whether the dog is performing correctly the proposed training activities offered by the system.

In recent years, ACI technology has also considered how wearable technology can improve animal welfare in farming scenarios. Haladjian et al. [105] studied motion sensors inserted inside a pig's ear in order to classify the pig's physical activities into 'walking', 'eating' and 'resting'. This ongoing work would enable veterinarians to keep track of free-roaming pigs and the pilot study showed the approach was viable and would even enable the tracking of pig activities with an accuracy of $95.8 \%$. In a similar manner, Haladjain et al. [104] implemented and evaluated a wearable device to be attached to a cow's hind left leg. This device could detect gait anomalies in cows in an effective way for large cow populations. The proposed device builds an individual model of the usual walking pattern of a cow during the first minutes of use and detects deviations from this model, reaching a $91.1 \%$ of accuracy. Recently, Carpio et al. [40] proposed a novel smart farming system and application framework based on wearables and cloud computing, with an emphasis on animal welfare features for cows and pigs. As opposed to haptics, 
it can be observed that wearable interfaces not always imply that the animal is an active user of the technology. In some scenarios in which the technology is placed on the wearable for sensing purposes, the animal might not be required to make any kind of interaction, other than being just a wearer, and thus is clearly not aware of the data exchange that is happening between the wearable technology and the data receiver.

As laid out above, the research and commercial space for wearable and vibrotactile devices has grown significantly in the last few years beginning with chicken and dog systems, and recently extending into livestock and farming scenarios. These systems, in general, are aimed towards working and service animals, focusing on control and data exchange. However, there is clearly a gap between using wearable interfaces to quantify the animal's actions, and pairing this data with vibrotactile interfaces to allow a two-way feedback from the animal to the human and vice versa. In addition, as more of these devices are deployed it is essential to continue studying how the use of haptics, wearables and biotelemetry devices affects the behaviour of the animal wearer.

\subsubsection{Olfactory Interfaces}

Grounded within the principles of animal-centred design, Johnston-Wilder et al. [137] (Figure 2.6) and Mancini et al. [180] have created interfaces to provide supplementary information, in olfactory detection of cancer by dogs, using pressure plates. These studies, building from dogs' olfactory work, have found that an olfactory system is a possible interaction method within ACI, highlighting the potential of this approach as it allows for the dogs' natural behaviour while sniffing the samples. Analysis on the pressure patterns from the sensors allowed to distinguish between positive, negative and uncertain samples, removing errors derived from human interpretation of the dog's interactions. Currently these authors are further exploring learning algorithms to implement these pressure patterns as a recognition tool.

Lawson et al. [160] has also proposed olfactory systems for dogs' socialization within a speculative design fiction system they term "the internet of dogs". These design fiction scenarios allow for dogs to use smell as a communication system by focusing on the production and identification of odours, suggesting that dogs' primary sense of smell could be used as a CAPTCHA within this "internet of dogs". During this work Lawson et al. [160] noted previous speculative designs that proposed the use of dogs as olfactory authentication mechanisms within ordinary technology systems (e.g., ATMs), questioning the user-centric values of future olfactory technology.

Moving away from dogs, Kobayashi et al. [153] describe a system for humananimal interaction in wild environments, for deer. The proposed system consists 




Figure 2.6: Olfaction cancer detection system [137]. Photo courtesy of The Open University.

of a remote-controlled rotating table that the user manipulates using a screen interface. The table, surrounded by surveillance cameras, is located in a wild environment, and has a deer cracker made of rock salt placed upon the table. The system is intended so that the smell of the rock salt would attract wild deer, creating a remote interactive experience with wildlife for the human user.

In summary, olfactory technologies as input interactive mechanisms in ACI have currently only been studied within service dog interfaces and speculated about with dogs and deer. The exploration of olfactory technologies for animals introduces many unknown questions, not only about the animals' interpretation of the interface, but, as Lawson et al. [160] expresses, about "what information the dog has understood, or transmitted". As technology develops further in this field, so will the variety of animal users that this can be applied to, and the instances in which this can be used grow, even if we do not completely understand the animals' reasoning fully. 


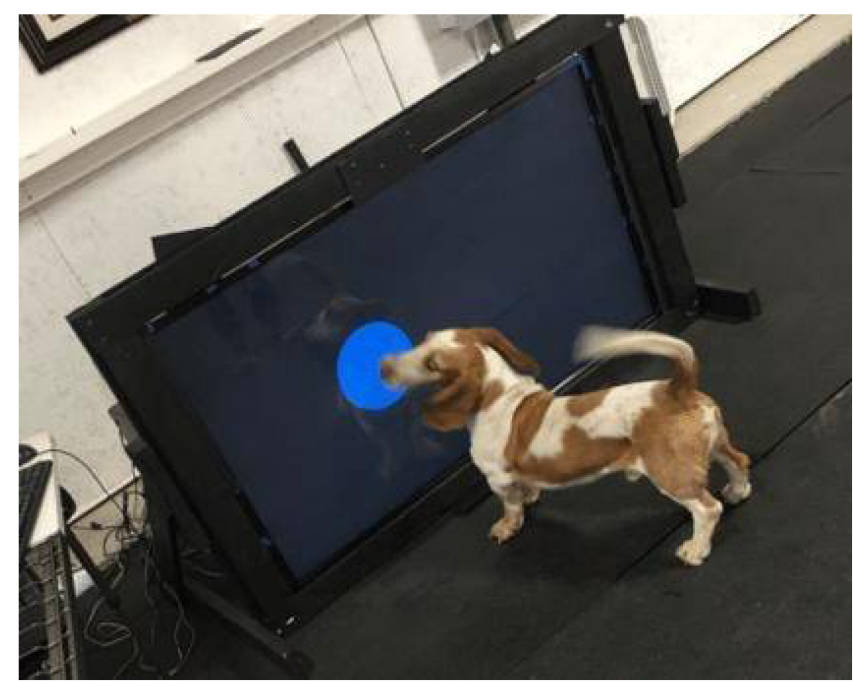

Figure 2.7: Dog training to click on points on a touchscreen interface [369].

\subsubsection{Screen Technology}

Whilst many novel interface devices are used in ACI, the classic visual and touchscreen interfaces contribute a large proportion of animal-computer research. Research in this area has included using tablets as UIs for dogs watching videos [17], screens for remote notification systems [369] (Figure 2.7), tablet games for cats [351] and orangutans [342] (Figure 2.8), wall interactive devices for pigs [5] and investigations on the usability of screens for dogs [118].

Screens can be configured solely as output technologies or, more recently, as input/output using a stylus or touch. Many enrichment activities conducted at zoos and sanctuaries with orangutans have involved the use of touchscreens or tablets, such as in the Apps for Apes project [10]. This project has also been used in several zoos around the world, including with orangutans at Melbourne Zoo [342]. The most common scenario for this sort of interaction is where a human keeper holds the tablet in front of the apes' enclosure so that an orangutan can touch the screen with its digits through the mesh. The human keeper typically encourages the orangutan to engage with the different apps offered in the touchscreen (Figure 2.8). Orangutans have also been shown to be able to use visual touchscreen interfaces with a stylus [276] within a musical preference study. In this work, orangutans could use a branch within their enclosure as a stylus to select one of the two halves of a screen, and depending on the side chosen, a different musical piece was played. Importantly, within this study it was found that 


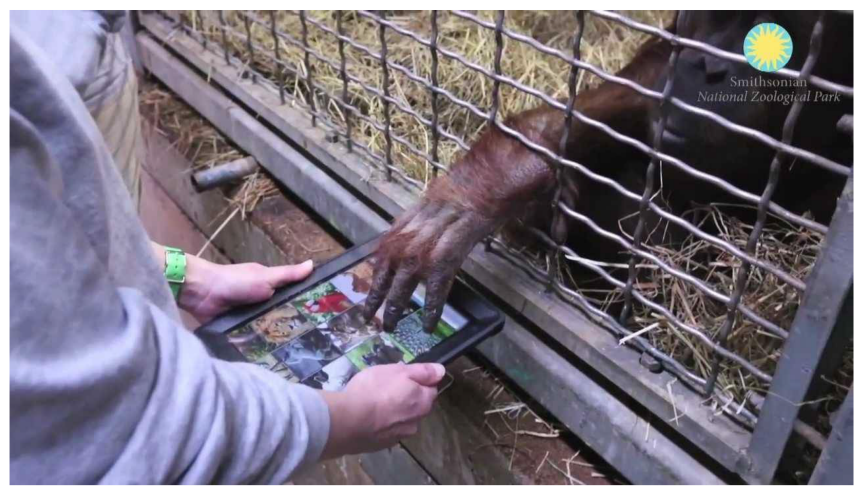

Figure 2.8: Apps for Apes: An orangutan using a touchscreen [342].

orangutans often rejected the technology. Allowing natural interactions with technology exposes many interesting things, including non-use. Wirman [361, 364] has extensively explored how orangutans would naturally interact with screenbased technologies, listing all the different ways orangutans explored the tablets or screens they had lying around their environment. These included not only touching the screen with their digits (fingers), but also using sticks, licking with their tongues, and pouring liquids over [364]. Another example is the work conducted by Perdue et al. [240], who installed a touchscreen in a naturalistic tree structure inside the orangutans enclosure at Zoo Atlanta. This work studied the effect of the touchscreen in orangutans' behaviours and also assessed the human visitor perceptions of the animal-computer interaction. At Indianapolis Zoo, touch panels have been used with orangutans to provide them with enrichment activities while at the same time allowing for the study of cognitive abilities in different tasks [185]. These authors also created an installation in which humans could interact, and play together, with the orangutans in a shared touch panel (Figure 2.9).

Playful interactions between animals and screen devices within ACI have also been studied for non-primate species, including pigs, cats and dogs. Alfrink et al. [5] proposed a novel interface for pigs' enrichment, in which pigs and humans could remotely interact via a giant touchscreen located in the pigs' enclosure. The human would remotely control a visual element that would appear in the pigs' screen, so that the pigs could follow it and touch it with their noses creating a combined way for humans and animals to use a screen interface together. Building from this human-animal screen interaction, Westerlaken \& Gualeni [351] developed and evaluated a tablet-based game for cats coined Felino. Felino was designed following an animal-centred perspective, in which the interaction could be adapted on the human side towards the ongoing perceived experience of the cat players. Baskin et al. [17] have studied humans' perception of dogs' interactions with tablets con- 


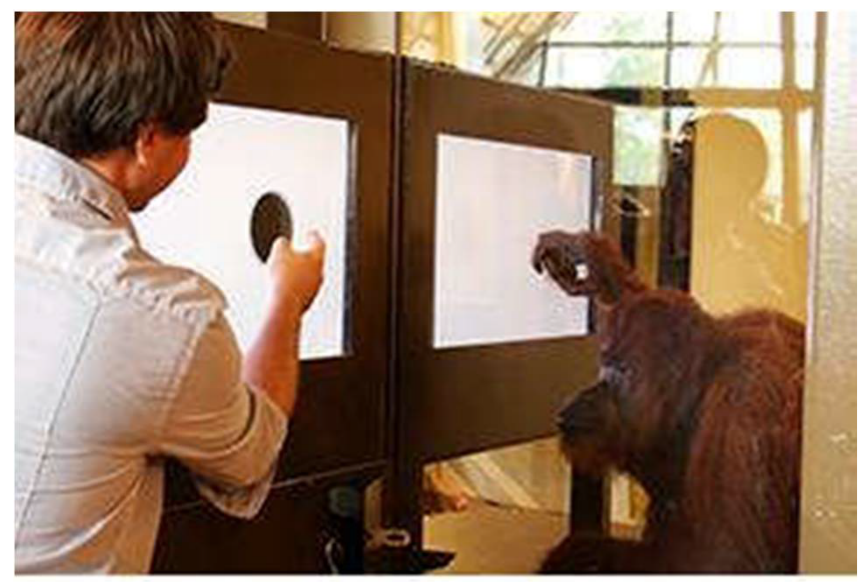

Figure 2.9: Human and orangutan playing together with a touchscreen interface [185].

sidering the screen device as an output interface in which dogs could watch videos. Their study built from Hirskyj-Douglas and Read's [117] work on increasing human perception of dog behaviours with screens using the Dog Information Sheet (DISH). Baskin et al.'s [17] results acknowledged that not all perceived interactions could be considered playful and that careful consideration needed to be taken into account when designing this kind of interactive experiences. This issue has previously been highlighted in Lawson et al.'s [161] work on speculative design with dogs and cats where it was found the human would often trust the judgement of the technology over scientific judgement.

Zeagler et al. [370] examined touchscreen interfaces with dogs for an alert interface and pointed out that affordances should be investigated to make touchscreens more usable for these animals i.e., the appropriate use of colour and understanding spacing between activation 'dots'. More recently, Zeagler et al. [369] presented work around the training methods of implementing these systems with dogs using touchscreen (nose) interfaces. Zeagler et al. [369] sought to train dogs to connect two dots on a touch screen interface by firstly training a dog to touch a single dot and then training the dog to slide the nose between two dots to create a dog alarm system similar to Robinsons et al. [281] tangible work mentioned in Section 2.3.2). Their work presented guidelines for future touch screen interfaces around the type of interfaces (non-projection monitors), the target distance and size (at least 3.5 inches) and on the best training paradigms (shaping for training touch and backchaining for sequential tasks) towards getting dogs to achieve the best behaviour modification required to use the technology. Importantly, Zeagler et al.'s [369] work found that first contact touch screen interfaces are easier for dogs 
to use and understand than lift-off interactions adding towards the design considerations and training methods in the space of dog screen interfaces. The authors sought to further explore this space through fully training dogs to use such screen systems to 'call for help'.

Whilst touchscreen design is clearly interesting, ACI has still not really understood the extent to which animals attend to, and can interpret, what is being shown on screen-based displays. Dogs' attention to screens has been previously explored in studies in animal behaviour that have tracked vision [308, 357] or touchscreens [339] with static images. Extending this study into moving media is beginning to be explored within ACI through workaround screen interaction with artificial presences and virtual reality systems [230] and with methods to analyse multiple screen systems [118]. Hirskyj-Douglas et al. [118] created a method to test a dog's viewing habits, favouring and following between three screens with initial findings indicating that dogs do have a media preference but also low attention times with screen devices. Hirskyj-Douglas et al.'s [118] core contribution within screen devices however, was that their method allowed for dogs to not attend to screens unlike previous work [308, 357], echoing out Ritvo and Allison's [276] findings of animals rejecting technology.

Ohta et al.'s [230] work in progress research plans to use interactive video interfaces to investigate the visual feedback loop (with partial depth cue perceptions) effect on animals' behaviour to investigate the animal attachment between dogs and robots. This research builds on Kerepesi et al. [149] previous work on visual communicative signals between dogs and humans and cats and humans, and Pongracz et al. [283] work on projecting human images to signal dogs. Wallis et al. [339] take these ideologies further by seeking ways for screens to provide mental stimulation for aging dogs in an animal welfare stance. The mental stimulation is designed by means of touch screen discrimination between two objects giving positive or negative feedback for cognitive enrichment. Delineating from this, Hirskyj-Douglas \& Read [114] built methods for dog driven screen devices for dogs in their home by using IR proximity sensors to detect a dog and displaying media o the dog when present. They used this method as a way of flipping the paradigm on normal media devices by allowing the dog to control, and choose, if they wished to use the system.

There is therefore, a growing, nonetheless limited, body of research, as demonstrated above, to investigate and map animals' requirements towards visual interfaces. One way that requirement gathering, and evaluations have been conducted with visual interfaces in HCI and animal-technologies is through tracking technologies, as will be described in Section 2.3.6. Another core area of discussion regarding screen technologies is whether the interaction with the interface remains user-centric from the animal perspective depending on its species. For example, screen technologies for zoo enrichment for great apes do not usually allow the animal to hold the device, and screen technologies for animals currently normally 
require training influencing the animals' ordinary behaviours. Nevertheless, screen technologies have been one of the most researched areas within ACI, with the flexibility and prolific occurrence of these interfaces offering insightful results to inform the field of ACI and HCI for unordinary users of screen systems. As new perspectives and methodologies are incorporated, future applications and explorations on these devices remain to be discovered.

\subsubsection{Tracking Technologies}

The notion of eye tracking, as a way to examine focus, has been around since the 1800s where people conducted eye movement studies from observations with Edmund Huey progressing the field in 1908 using contact lenses on the subject's eye with a hole for the pupil and the use of aluminium pointers [39]. In the late 1990s early 2000s, eye tracking technology was expanded towards animal users, focusing mostly on primates and dogs; some of this work involved surgical interventions [212]. Body, face, eye and gaze positioning have played a part in understanding human and animal behaviour in ACI through tracking gaze [308], body posture $[190,257]$ and automated face reactions [162] similarly to HCI [134, 261]. The advancements made in HCI tracking technology have not yet been fully exploited in ACI technologies, but there is an increasing corpus of ACI studies regarding animal's tracking in horses [221, 223], cats [255, 257] and dogs [190, 308, 357] (Figure 2.10).

Williams et al. [357] wanted to increase spatial accuracy for laboratory settings by using mobile head mounted, video based, eye-tracking system achieving in their work an accuracy of $2-3^{\circ}$. Sompii et al. [308] took a different approach than Williams et al. [357], by, instead of training a dog to wear a mounted system, training a dog to rest its head upon a headrest to achieve contact-free eye movement tracking. Unlike Williams et al. [357], Sompii et al. [308] used pictures rather than treat location tracking. Sompii et al.'s [308] research provided evidence that dogs focus their attention on informative regions of the images where their gaze fixation depended upon the images category (human, dog, shape and letter). This discrimination of images lead to suggestions that dogs can discriminate images of different categories corresponding with Farago et al. [67] who found that dogs consider natural objects more interesting than abstract ones. Somppi et al. [308] did comment however, that they cannot yet draw any conclusions as to whether the attention of dogs was directed towards stimulus features or semantic information or a mixture of both opening up questions in animal tracking around the impact of the complexity/simplicity of the image in regard to findings. This work did delineate towards species-dependant behaviour [102, 264] when viewing faces towards a more natural setting moving Williams et al.'s [357] goal of naturalistic tracking forward. Research into animals' cognitive processing of technology is particularly needed in animals where welfare is of concern because they cannot vocalise opinions and choices [173]. Animals can be trained to use tracking 


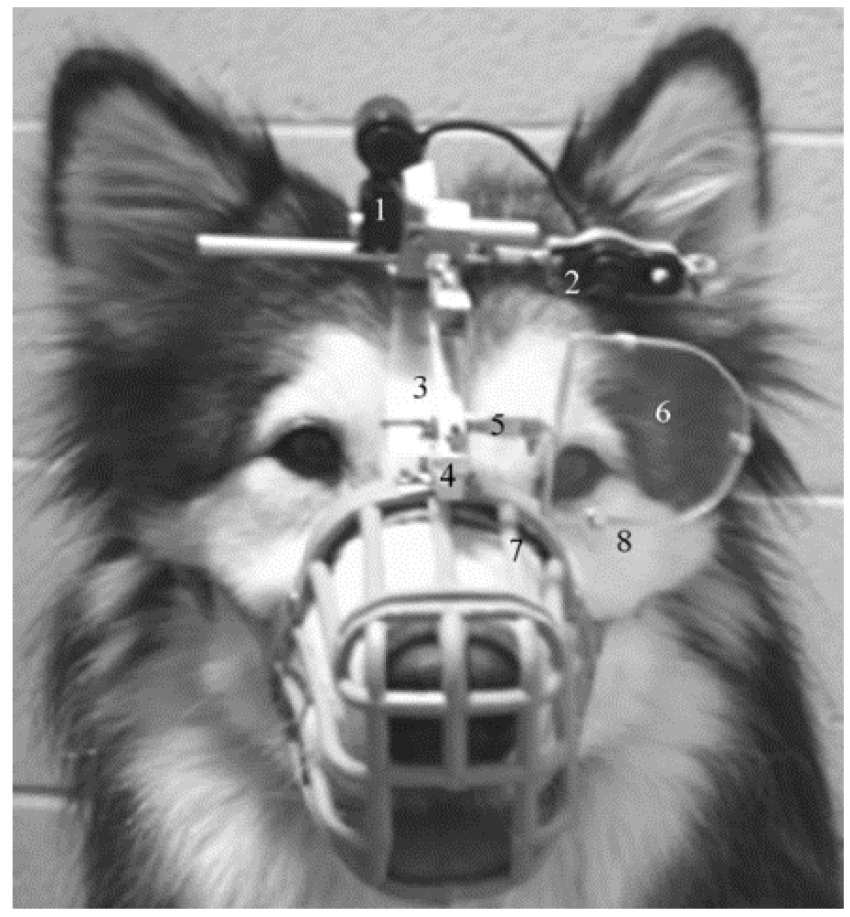

Figure 2.10: Using a head-mounted, eye-tracking system with dogs [357].

systems [308] or can be tracked wearing head-mounted systems [357], but both these strategies are known to influence their ordinary behaviour, which is the very thing, ironically in these studies, that researchers are typically aiming to measure as noted in Hirskyj-Douglas et al. [118] work mentioned in Section 2.3.5.

The constraints and difficulties of tracking technologies that limit the animals' natural behaviours leave a space open within animal-computing to draw back to the original observational tracking methods in HCI to allow animals to explore technology in ordinary ways, merging early human methods with current usability methods. ACI has recently proposed image-based-human-interpreted recognition systems with horses [221, 223], orangutans [343], giraffes [56], cats [255, 257] (Figure 2.11) and $\operatorname{dogs}[113,190]$.

These non-intrusive tracking systems vary in how they operate with some using image shape recognition [257], feature and posture recognition [113, 190, 223, 257], motion recognition [343], proximity [114], and point recognition [308, 357]. Pons et al. [257] used a Microsoft Kinect depth sensor facing down from the ceiling to record cats' naturally behaviour when playing. This data was used to create a 

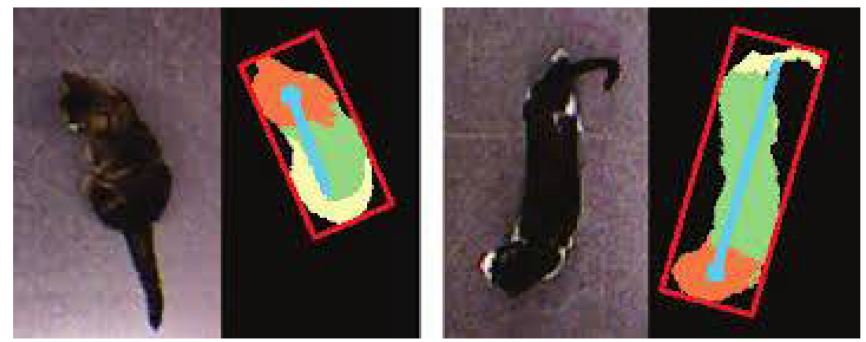

Figure 2.11: Tracking cats using depth measurement via an Xbox Kinect to detect posture [257].

non-wearable tracking system capable of recognizing cats' postures (sitting, semisitting, walking, standing, jumping and turning), body parts (head, body, tail) and orientation (Figure 2.11). The tracker used the average area of the cat's contour, number of pixels and average depth for each cluster to classify the image. The performance of this non-wearable tracker has been extensively analysed in a followup study [255] in which authors compared several machine learning classifiers and a greater set of features for the classification.

Building from Pons et al. [257], Mealin et al. [190] (Figure 2.12) used a Microsoft Kinect depth sensor as well for posture and feature recognition for dogs. Mealin et al. [190] used average depth values and aspect ratios of bounding boxes around the animal, rather than clustering features, for the classification. Both these systems, however, are still semi supervised, where in the case of Mealin et al. [190] multiple images of the background must be included within the training data set to gather better depth reading when comparing singular images, simplifying background separation from the dog. This requires an expert user to implement these systems. Several works, especially in the area of animal behaviour, have used just colour cameras to detect animals' shapes and track their movement without any posture detection, such as with pigs [228], dogs [7], chickens [164] or mice [99].

North et al. [221, 223] have proposed, and are currently developing, a videobased automated behaviour identification software tool for observations of both horse-to-horse and horse-to-human interaction coined HABIT. In 2017, North proposed to extract salient features, such as the horse's ears, in order to start the detection of the horse in real scenarios and from a variety of viewpoints. Also taking a very animal-centric approach, Webber et al. [343] created and evaluated a tracking system for orangutans in a zoo environment. The interactive system consisted of projecting images on the ground of the orangutans' enclosure, while a depth sensor tracked the movement and touch of the orangutans over the projections. This system aimed for enrichment purposes allowed a more natural exploration of the technology from the animals' point of view. Another approach, proposed by 


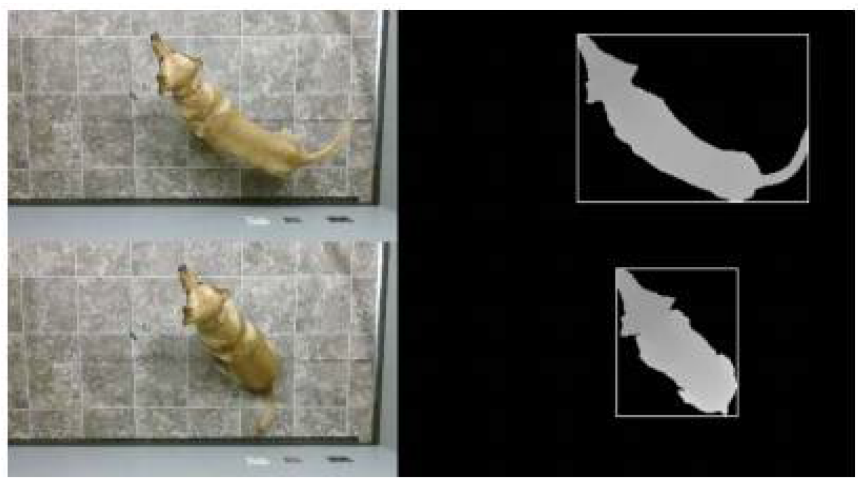

Figure 2.12: Tracking dogs using posture recognition via an Xbox Kinect [190].

Dong et al. [56] for zoo environments, is the use of a single thermal vision camera for giraffe identification and tracking in an unconstrained environment. This work detects giraffes within the image through their body temperature and distinguishes them from other animals by applying machine learning techniques. This proposed system is capable of tracking the movement patterns of giraffes within their enclosure during day and night, allowing for 24-h monitoring.

The space for tracking animals is sparse but growing towards untrained and unsupervised off-body systems, as much as vibrotactile systems are growing into on the body systems. From this review, it seems that whilst tracking technology has a growing use within ACI, particularly in image and posture recognition, there is still a missing gap of research that needs to be conducted to ensure its usefulness towards the animal's normal behaviour. Specifically built animal contact-free and training-free versions of eye tracking systems still do not exist. In addition, complex behaviours cannot be inferred from basic posture tracking, which makes it difficult to build a system capable of fully reacting to the animals' intentions. There is clearly a space open within ACI for studying non-wearable tracking of animals' behaviour, in which interdisciplinary approaches and knowledge from animal behaviour are essential in this matter.

\subsection{Future of Technologies within ACI}

As this field is a relatively new area of investigation, there is much further work that needs to be done to iteratively test some of the technological interfaces, designs, systems and models outlined within with a larger dataset: of varying animals and breeds at different ages in different contexts and situations. In addition, several works within ACI that have not been covered in this literature review have focused 
on methodologies for, and the ethics of, designing and building technology for and with animals - this leaves an open body of work. As the technology around ACI grows, the results will inform the development and formal definition of specific methods for this discipline, drawing from conjoining fields such as HCI and animal behaviour. In return, these methods will greatly help to advance the development of new technological devices and systems with a further grounded animal-centred perspective. The technology informed results described above are placed within the time and context of the current technology, which with advancements, turning towards the pet, zoo and farm specialties and computer interaction interfaces, a few of the obtained results may significantly change over time.

The research works that have been discussed above within this literature review have been received with interest within the community and where presented; however, the acceptance of these views varies dependent upon the community's set paradigms. The ACI community embraces the animal-centric technologies and results gathered as the philosophical approach fits within the community paradigms laid out within the ACI Manifesto [173]. To the HCI community, they embrace the recognition of method transferences, particularly between animals, children and other non-verbal users to elicit new methods [120, 174]. To the animal research community, the animal-centric approach is often considered in a more flexible way. For example, training is frequently carried out and the initial results are considered as a source of information, in contrast with more goal oriented systems [260, 349]. To animal owners and those who look after animals, the potential outcomes of the ACI field hold promise for entertainment and enrichment possibilities as shown through the recent growth in pet technological products towards fostering monitoring [72] and playful robotic systems [262]. However, the animal-centred perspective of ACI studies is not always materialized in industry designs, which requires for careful considerations when deploying pet technology at homes, into our zoos and farms. In this regard, both industry and the ACI community still need to find a way to complement each other and work together towards ensuring animal welfare.

The rest of this section sets out the research agenda for future work which would have implications towards each of the communities outlined above. This research agenda forms an important contribution of this literature review and is drawn out through the noticed gaps informing both novice and experienced researchers within ACI. 


\subsubsection{Animal-Driven Devices for Enrichment and Work}

Within modern society many animals, such as pets and farm animals, often spend time alone. Devices that are designed for animals to support these alone periods could potentially provide a usable platform for developing enrichment devices. This is not to state that enrichment is a sole process; technology could also align with the human-animal bond to enrich that play, and there are already several works and projects devoted to this line of research [257, 343, 361]. Most of the works proposing enrichment or playful technology for animals have been focused on pets and zoo animals, while studies centred on farm animals have been mostly oriented towards welfare and data exchange (see Figure 2.2). However, farm animals which are arguably the most mistreated animals within modern society could also greatly benefit from playful technological interventions, which can certainly be another way of improving their welfare. The ACI community is slowly beginning to explore these enrichment opportunities [213].

One of the most studied species within ACI is dogs, with one of the biggest growth areas of ACI being devices to support a dog's work. This has motivated that, in some ACI technologies, rather than the human being in control of the system, the animal is trained towards using the system. This training varies from the animal making simple behavioural choices of activating a device when faced with certain situations $[89,281,369]$ through to being trained to sort something $[137,180]$. On the other end of the scale, there are less constructed and more exploratory interactions where an animal is presented with technologies and their behaviours documented, such as interactions with robots [87, 260, 349]. A future step could be taken in ACI to deeply explore the idea of animal centred methods $[116,173]$ to allow technology to be shaped around animals' affordances. It is in this way that the technology used will become yet even more suitable for the animal. Building from previous investigations in button systems [181], enrichment for zoo enclosures [76], or screen systems [118], one of the promising mechanisms to explore animal-centeredness could be the design and development of animalactivated systems. For example, tracking systems could be useful to account for natural occurrences of animals initiating an interaction through observation and learning.

\subsubsection{Investigating What Is Interactivity in Animal-Computer Interaction (ACI)}

At the beginning of this literature review, comments were given on the feedback loop between animals and systems. One of the key areas for ACI is to investigate what it means to have interaction between animals and technology. For instance, depending on the focus of the research, animals have been considered to be wearers, as in the case of biotelemetry devices, to fully interactive users, such as within some playful technologies. However, there is a whole range of interactivity levels, 
participation models and discussions around whether an animal is actively or inadvertently using some technology, and these have each yet to be scoped within ACI. As an example, haptic interfaces such as vibrotactile vests provide an interaction that the animal receives, with the animal initially not knowing what caused the system to be triggered and instead learning the triggering mechanism through training schemes focused around shaping the animals' behaviour. There are also cases, such as Lee et al. [164] or Van Eck \& Lamers [61], where the animal receives the output produced by a human user, without any control on the situation, which might not arguably be an interaction from the animal's stance. On the other side of the spectrum, involuntary participation of the animals within systems, such as the idea of consent within ACI [173], are being introduced into ACI where the animal is free to choose to interact or not [118].

Currently, little is known about the reasoning behind an animal's interactions with technology (the gulf of execution) and what the term 'interaction' means in the field of ACI, although this is beginning to be explored and discussed [74]. This could be further investigated through looking at what an animal's usability is through their attention towards technology and behaviour while using systems, and then aligning systems to allow for these actions. Long-term deployments of the technology would also help to observe these spontaneous behaviours in a lessconstrained scenario than prefixed experimental sessions. All these elements could help further build up a stronger picture of interactivity in ACI.

\subsubsection{Ethics and Agency in Animal-Computer Interaction (ACI)}

Whilst this literature review does not aim to tackle in depth the notion of ethics and agency within animal-computing systems, these attributes are inherently interwoven into technology interfaces used in ACI. As Mancini [175] advocates in her welfare-centered ethics framework there is a case in ACI for moving beyond existing regulations and guidelines in an animal-centric approach. This framework, like Hirskyj-Douglas \& Read's ethical framework for dogs [115], focuses on obtaining mediated and contingent consent from the animal. Alternatively, Väätäjä [324] takes another viewpoint upon ethical technological implications for animals seeing ethics as a welfare issue, and therefore motivating her framework within the need for mediating relatedness and intimacy with technology drawing from the ' $3 \mathrm{R}$ 's' in science of replacement, reduction and refinement [325].

Intrinsically tied into this discussion is consent and animal agency, that is the ability of the animal to act independently and to make their own free choice. Whilst Mancini [175] argues that technology blurs the boundary between animal and human agency, as the lines between user and interactors are obscured, HirskyjDouglas and Read [114] advocate for animal agency through exploring technology interventions that aim towards animal-driven devices. This stance is in an aim towards free choice (consent) from the animals themselves to give the animals 
further agency within technological interventions, which situates this conversation between the ethical morality of working with animals and the human drive to gather data [115].

Drawing back to technological instances, whilst ACI has the potential to significantly contribute towards forming ethics of animals' situation within and using technology, the current landscape of computer systems, as explored above, has varying degrees of ethical implications and agency for animals within technologies. For instance, not all the technologies explored within this manuscript allow for consent, nor understand consent in the same way. Thus, the future of technological devices for animals in computer systems is closely drawn in relation to ethical and agency propositions towards methodological conditions for both shaping future technologies and fostering animal-centric approaches. This creates a juncture in ACI to explore giving the animals varying degrees of agency within technology localities to shape the future of ACI systems under a common understanding of welfare implications and ethics.

\subsubsection{Moving Beyond the Human-Animal-Computer Void}

Perhaps due to the novelty of the field and the fact that the ACI community still has more questions than answers, most of the works reviewed in this manuscript are focused on a single animal interacting with a system. Several works have also addressed the role of technology in human-animal relationships, as well as humanmediated systems for animal use. As suggested in Weilenmann \& Juhlin [344], Asplin \& Juhlin [11] and in this literature review, there is an opportunity to move ACI research from a sole animal-computer interaction and human-animal computer mediated interaction towards animal-animal interaction by means of technology. Whilst it is unknown at this stage what animals need and want in regards to computer technology for animal-to-animal communication, it is only through exploring these areas that new light can be shed. Animals are often put within situations which are not ideal for their well being due to human circumstances i.e., shelters, rescues and left in homes, which isolate their contact from other animals where technology interventions could intervene aiding both the human career and the animal themself.

The animal-to-animal internet has been used on a technological level for internet communication sharing capability as a method to maximise technology performance by allowing the systems worn by different pigeons to communicate [152]. This has been irrespective of the animal to animal interaction themselves however. Whilst Reiss et al. [271] have suggested the interspecies internet to allow all animals to communicate online and Lawson et al. [160] suggests the internet of dogs via olfactory systems, this ideology has yet to be brought into fruition beyond ideologies and design fiction to connect animals mediated through computer systems. Perhaps this is the next step for animal-centricity: by building up technology 
systems to allow the internet of animals to evolve building ACI solely off animal requirements. This would be allowing, in some sense, to surpass the barriers within ACI put up by humans. This narration includes the exploration of multispecies computer mediated interactions, i.e., animal-animal interactive systems.

It is acknowledged however that even within animal-animal technology systems that are animal-centred the human is inherently part of the interaction paradigm as they are the ones building, initially designing and informing part, if not all, of the system(s) and interpreting the behaviour as an output. However more efforts could be placed towards having the same responsibilities and actions for both the human and the animal during the interaction phase, giving more voice and control to the animal to guide the interaction and in defining how they want to interact with a human $[258,361]$. Whilst it is understood that currently the human-animal ACI user space has not yet fully been explored, this is not necessarily a prerequisite to animal-animal exploration but is sure to benefit the diverse multispecies use.

\subsection{Conclusions}

This thematic literature review provides an outline of the ACI field concerning the technologies involved. As demonstrated in this review, one method for analysing these technologies is through their use; tangible and physical, haptic and wearable, olfactory, screen technologies and tracking technologies. This is just one way of exploring current literature, yet it is necessary to approach the foundational work in the field in order to reflect on the acquired knowledge. We hope this framework will help building up new perspectives over ACI technologies and help to identify promising opportunities for further development. Many of the technologies explored above have potential within various areas of ACI from assisting with human health technologies, giving animals more control, enabling data exchange between animals and humans, assisting working and service animals and monitoring animals in both our own environments and the natural habitat.

There are currently few, but a growing number of, technologies in ACI often stemming from and adapted from HCI and ethology. This literature has identified technologies used in ACI to support both human to animal, animal to human and animal to robotic communication via computerised systems. In particular, this thematic literature review notes spaces within tangible and physical, haptic and wearable, olfactory, screen interfaces and tracking technologies. In tangible and physical technologies, a change was noticed from traditionally investigating cognition and behaviour towards looking more closely at the interaction paradigm, with particular systems expansion in the zoo and pet fields for new technologies. Gaps were observed within tangible and physical systems to investigate new training paradigms so animals can use systems more efficiently, and to look further at how animals respond to the interfaces that they hold and use. For wearable systems it 
was noted that ACI is expanding into wearable systems in order to quantify the animal's behaviours and actions, providing supplementary information away from tracking systems. Haptic systems are expanding further into providing a communication mechanism between the animal and the human to investigate more deeply the feedback loop. Another space identified within these two fields are in relation to pairing these systems together to provide quantified and enhanced communication systems. In olfactory technologies, currently only dogs and deer have been investigated, and only in one implemented system, leaving spaces open to investigate this interaction mechanism in other species and further for dogs. In visual screen interfaces, there is a gap to map the animals' requirements towards these interfaces, as mentioned through tracking technologies. It was also noticed the need for these screen technologies to be more user-centric and to vary by species drawing from the field of HCI. Lastly, in tracking technologies there is a gap and a move in ACI tracking methods towards unsupervised and untrained off the body instances, particularly towards its usefulness for the animal user and to quantify the animals' behaviour. As suggested throughout the various sections of this literature review, there is a need for a further interdisciplinary approach within ACI technologies to ground the field forward within animal cognition and behaviour not just within the computer interaction space.

There are, like Jukan et al. [143] mentions, technical and economic challenges to overcome, but these have to be frame worked and mapped together to create a more foundational field knowledge from which to build for both new and expert researchers and developers of ACI technologies. As a research field, ACI is embedded within the research mentality towards positive animal welfare. It is instead a question of the boundary of how much implementation of technology, the application of these developments and the interaction paradigms that need to be carefully explored.

\section{Acknowledgments}

The work of Javier Jaen and Patricia Pons is supported by the European Development Regional Fund (EDRF-FEDER) and Spanish MINECO with Project TIN2014-60077- R. Patricia Pons also receives support from a national grant from the Spanish MECD (FPU13/03831). The authors would also like to thank Patrizia Paci for her contribution during the beginning stages of this review. 


\title{
Chapter 3
}

\section{Envisioning Future Playful Interactive Environments for Animals}

\author{
Patricia Pons, Javier Jaen, Alejandro Catala
}

ISSI Group, Department of Computer Systems and Computation (DSIC), Universitat Politècnica de València, Valencia, Spain

Published in A. Nijholt (Ed.), More Playful User Interfaces. Gaming Media and Social Effects, pp. 121-150, Springer (2015). doi:10.1007/978-981-287-546-4_6

\begin{abstract}
Play stands as one of the most natural and inherent behavior among the majority of living species, specifically humans and animals. Human play has evolved significantly over the years, and so have done the artifacts which allow us to play: from children playing tag games without any tools other than their bodies, to modern videogames using haptic and wearable devices to augment the playful experience. However, this ludic revolution has not been the same for the humans' closest companions, our pets. Recently, a new discipline inside the Human Computer Interaction (HCI) community, called Animal Computer Interaction (ACI), has focused its attention on improving animals' welfare using technology. Several works in the ACI field rely on playful interfaces to mediate this digital communication between animals and humans. Until now, the development of these interfaces only comprises a single goal or activity, and its adaptation to the animals' needs requires the developers' intervention. This work analyzes the existing approaches,
\end{abstract}


proposing a more generic and autonomous system aimed at addressing several aspects of animal welfare at a time: Intelligent Playful Environments for Animals. The great potential of these systems is discussed, explaining how incorporating intelligent capabilities within playful environments could allow learning from the animals' behavior and automatically adapt the game to the animals' needs and preferences. The engaging playful activities created with these systems could serve different purposes and eventually improve animals' quality of life.

\subsection{Introduction}

The world's diversity of species is one of its most impressive characteristics. There are approximately 1.1 million of known animal species in the world ${ }^{1}$, each of them contributing and giving shape to the ecosystems we live in. However, as a consequence of this vast heterogeneity of animal beings, having a common way of communication between all of them becomes impossible. Even within the Homo sapiens species, some handicaps arise when humans with different cultures and/or languages try to communicate. Nevertheless, there exists one behavior present in the majority of animal kinds which seems to remove the communicative barriers among species, facilitating the interaction and creating strong bonds between participants: play.

Play is one of the most natural and inherent behaviors among animals. In Huizinga's own words [131]:

"Play is older than culture, for culture, however inadequately defined, always presupposes human society, and animals have not waited for man to teach them their playing."

As Huizinga points out, animals do not need to be taught to play with each other or with humans. For them it stands as a natural activity which may have several purposes that are not yet completely understood [19]. In fact, one of the main aspects of play is that it is fun and this is the main source of motivation for all sorts of animals, including humans.

This aspect, being fun, has motivated humans not only to play but to design artifacts that make the play activity even more attractive. The nature of human play has therefore evolved with technological innovations from primitive stone skipping to modern interactive electronic games. However, in this hominid evolution giving rise to what Huizinga called the homo ludens and some call today homo ludens electronicus, other species have been left behind. This is the case of animals, as animal play has not experienced yet this digital ludic revolution in the same way as human play has.

\footnotetext{
${ }^{1}$ http://www.catalogueoflife.org/annualchecklist/2014/
} 
This chapter firstly describes the factors which led to the emergence of a new technological trend focused on animals as the target users of digital systems, explaining how animal play could be of great importance in this new research field. Secondly, a review of existing work on technology-mediated interaction with animals is presented, with a specific discussion of previous playful digital games for animals. Based on this review, we propose a new and more flexible way of understanding animal playfulness with digital systems: intelligent playful environments for animals. A conceptual development framework for these systems is defined, presenting an analysis of existing playful games for animals under this framework. This analysis will help to detect lacks and needs in terms of digital playful interfaces for animals. Finally, application scenarios, emerging issues and opportunities for interdisciplinary research are described for further exploration.

\subsubsection{Animals as Target Users of Digital Systems}

Since the emergence of Human Computer Interaction (HCI) as a discipline, the benefits that HCI applications and studies have brought to human well-being are countless. Understanding how humans interact with digital systems has allowed researchers and developers to design and build innovative and more natural interfaces, improving the user experience and lowering the gap between the virtual and the real world. More specifically, the contribution of HCI studies to the evolution of human play has been of extreme importance. HCI studies have allowed us to build digital devices which enhance our playful experiences, by making them more immersive and realistic: high performance portable video consoles, joysticks, motion sensing devices, technology for augmented reality scenarios, etc.

In the last years, we have seen how electronic devices meant for humans have been tuned or adapted for animals to play with them. Sometimes, even animals by themselves get interested in the devices around them and start using our digital gadgets in a way we would never have imagined. In Fig. 3.1, a dog plays with an electronic ball, called $S p h e r o^{2}$. This commercial device is controlled by a human, who uses a smartphone or tablet application to make the ball move while emitting light. Both the movement and lighting factors cause the animal to really get involved in a playful activity chasing and touching the electronic ball. Figure 3.2 shows two orangutans in a zoo using an iPad application as part of the Apps for Apes ${ }^{3}$ initiative. Apps for Apes aims to provide stimulating activities for orangutans in zoos by allowing them to play with several iPad applications. There are applications for painting, playing the piano, exploring pictures, etc. A volunteer approaches the iPad to the orangutans' cage and holds it as long as the orangutan wants to play.

\footnotetext{
${ }^{2}$ http://www.gosphero.com

${ }^{3}$ http://redapes.org/multimedia/appsforapes/
} 


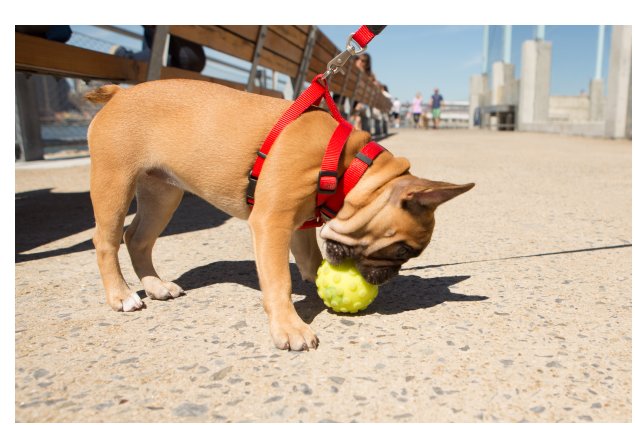

Figure 3.1: Dog playing with a Sphero



Figure 3.2: Orangutans playing with an iPad as part of the Apps for Apes initiative

Animals' interaction with our digital world is sparking our interest, as we begin to wonder whether they would be able to play with our human-centered electronic devices. However, little research has been done for developing digital systems specifically designed for animals in comparison with the efforts that have been focused on the construction of human-computer interfaces.

Recently, an emergent discipline inside the HCI community called Animal Computer Interaction (ACI) [173, 174] has started to shape. ACI principles are based on recognizing animals as target users of digital systems and developing computing technology specifically designed for them by studying how they interact with digital interfaces. Understanding animals' behavior with computer-mediated systems will help to develop systems more suitable for them, eventually improving both humans and non-humans quality of life. The ACI community is aware of the ethical issues derived from conducting studies with animals, and some guidelines have been proposed in order to ensure animals' welfare at all possible means [325].

However, ACI studies with animals have to face an important obstacle. If animals are going to be the target users of the systems, they have to be included in the design and development process, in the same way HCI includes human stakeholders in the construction of new interfaces. Generally, usability studies with humans rely on verbal or written communication for both giving instructions to the users on how to use the system, and for gathering information and feedback from the users about the system being evaluated. The impossibility of verbal or written communication with animals forces ACI researchers to look for other evaluation methodologies that allow them to communicate and understand the animals' interaction with the digital system. In addition, a psychological perspective is required in future ACI studies. The inability to verbally communicate with a group of interest can lead to erroneous conclusions when conducting studies based on choices [276]. If a subject is presented with two options, her choice could be based on the most desired option (which would be our assumption) or on the least 
aversive one (which does not mean it is a good option). Careful assessment should be performed in this kind of studies.

When looking for effective ways to understand how animals interact with computermediated systems, ACI applications should rely on their most natural and intrinsic behavior: play. The ACI community should take advantage of the animals' natural disposition towards playing and set playfulness as the basis of any system targeted at them. The use of technology-mediated playful experiences within the ACI field will provide engaging ways of conducting usability studies with animals, as well as an effective and worldwide understood way of communication between species - play. Moreover, advances in the ACI field will lead to the improvement of the digital devices used in playful experiences. These digital devices will become more and more suitable for animals as ACI insights are applied on their development process. As a consequence, a symbiotic relationship between ACI and animal playing will be created, giving rise to the era of the animal ludens.

\subsubsection{Playful Environments as Intelligent Ecosystems}

Several works have already addressed the design of playful experiences for humans [217], even analyzing the effects play has on human pleasure. According to $[50,51]$, the pleasures of play should be studied by considering multiple categories related to Creation, Exploration, Discovery, Difficulty, Competition, Danger, Captivation, Sensation, Sympathy, Simulation, Fantasy, Camaraderie and Subversion. However, these constituent elements of playful experiences that apply to humans may not be applicable to other species. They may need to be adapted for different types of animals or even be tailored for specific individuals or situational contexts in a transparent way.

Context-awareness, adaptation and transparency are the main building blocks of a currently growing technological approach known as Ambient Intelligence (AmI) $[219,345]$. The AmI research community seeks for the disappearance of computers as we already know them, providing users with seamless systems comprised of plenty of interconnected digital devices (ubiquitous computing). The communication between all these devices should be invisible to the user (transparency), and the system's main goal will be providing the users what they need taking into account their contextual situation (context-awareness). The infinite range of possible contexts and user preferences prevent developers from building a specific system for each situation. Instead, the solution lies on applying some sort of intelligence in a way that environments can learn from people's behavior and automatically adapt themselves to the context, even anticipating people's needs. For this purpose, diverse computing areas merge their efforts to come up with a fully integrated intelligent environment: artificial intelligence for activity recognition and decision making, sensing devices for monitoring users and environmental 
status, HCI advances to provide easy-to-use and useful interfaces, etc. As a result, AmI advances are helping to improve human well-being without any doubt.

There are certain parallels between humans' need for intelligent systems and animals' playful revolution. Playful experiences for animals will be diverse and should be tailored to their specific characteristics and needs. Thus, developing a specific playful system for each contextual situation will not be feasible due to the extensive range of possible scenarios. Playful environments could be provided with the same kind of intelligence that AmI proposes for human environments. Therefore, playful environments will have multiple digital playing elements, which could communicate between them in a transparent way for both humans and animals. These environments, which we call Intelligent Playful Environments for Animals (IPE4A), would extract knowledge about the animals inhabiting them, learning from their behavior and preferences. The environment could rely on this information to evolve and auto-adapt to the situation, creating suitable playful activities for each context without having to develop a specific system for each purpose/situation.

The next section will review existing works on animals' interaction with computer systems. This review will provide the reader with the adequate background to better understand the purpose of Intelligent Playful Environments for Animals.

\section{$3.2 \quad$ Related Works}

Despite ACI being a recent research field, studies concerning animals, their cognitive capabilities and the way they understand their surroundings have existed for a long time $[178,187,285]$. This section will analyze how computer mediated interaction with animals has evolved over the years, giving a closer overview on the recently emergence of technological playful interfaces for animals.

\subsubsection{Computer Interfaces for Animals}

In the 1970s, the LANA Project was one of the first attempts where computerbased interfaces were used to study the linguistic capabilities of chimpanzees [285, 286]. The system consisted of a keyboard with lexigrams, i.e. abstract symbols representing nouns, verbs, activities, etc. These lexigrams allowed the construction of sentences in an English-like language called Yerkish. Lana, in Fig. 3.3, was the first chimpanzee who learnt how to use the lexigram keyboard to communicate with humans. Touch screen computers and iconic keyboards have also been used in later projects with chimpanzees, such as the Ai Project [187], named after the female chimpanzee who pioneered the study. This project aimed to deepen into the cognitive capabilities of chimpanzees, and results suggested that they are able to outperform humans regarding simple memory tasks. Due to the DNA similarities 
between chimpanzees and humans, the interaction methods used in these systems were similar to the ones conceived for humans.

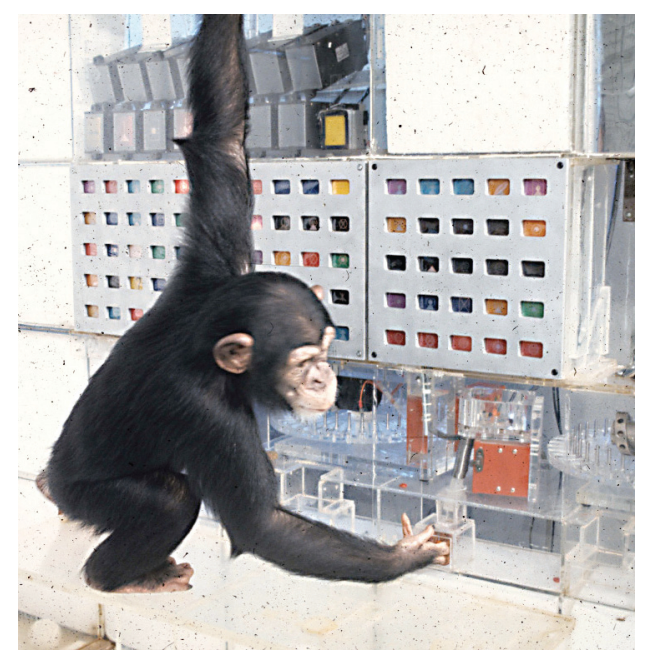

Figure 3.3: Chimpanzee Lana using the lexigram keyboard to request food (Image courtesy of. Dr. Duane Rumbaugh)

Communication between dolphins and humans has been another area of interest. The SpeakDolphin ${ }^{4}$ project uses a Panasonic Toughbook to introduce dolphins to the use of touch screens. Using this interaction modality, dolphins have to perform cognitive associations between real objects and pictures on the screen, selecting on the touch screen the picture of the object they are shown in real life. The next step would be adding symbols associated with actions in order to create a useful language interface.

One of the firsts attempts to apply HCI methodologies and User Centered Design for building computer interfaces for animals is Rover@Home [273]. This work grounds on the idea that the communication between humans and dogs is asymmetric. Therefore, the interfaces for dogs have to differ from the interfaces for humans in order to adapt to the communicative subject in each case. A computerbased system for clicker-training with dogs is presented, allowing humans to remotely train their dogs.

Wearable technology has also been used for improving remote communication between pets and their owners. This is the case of Poultry.Internet [164, 319], which proposes a tangible interface for poultry and humans at different locations. The chicken wears a special jacket (see Fig. 3.4) which emulates human touching

\footnotetext{
${ }^{4}$ http://www.speakdolphin.com
} 
when the human touches a pet doll. Also, the movements of the chicken are monitored and notified to the human using a haptic device that the human wears on his toes. In addition, computer-mediated tactile interaction with dogs has been studied, claiming that this interaction modality could help to alleviate dogs' stress and anxiety [324]. For the purpose of this study, dogs' behavioral problems and possible causes of stress have been analyzed. The main goal of this work is to provide a useful framework for improving the development of future wearable devices for dogs which emulate human touch.

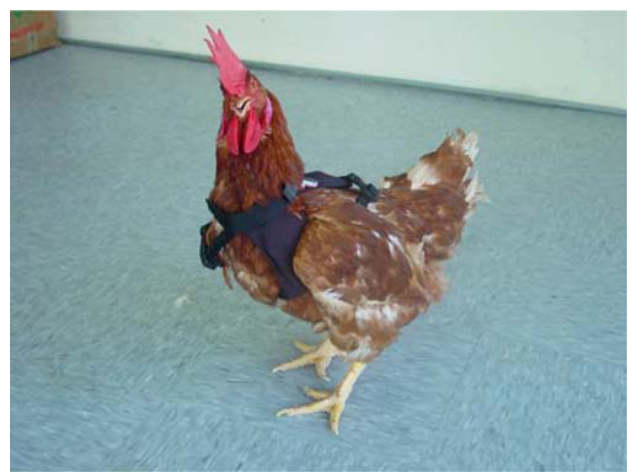

Figure 3.4: Chicken wearing a jacket which simulates human touching sensation (Image courtesy of Dr. Adrian David Cheok)

Some studies have reported how traditional human-animal interaction is affected by the use of technology, in this case, a positioning system for hunting dogs [235, 344]. This system allows hunters to follow in real-time the position of their hunting dogs. This additional information enriches the perspective the hunters have about the dogs' behavior. As a consequence of knowing where the dog is, hunters begin to imagine what the dog will be doing based on its movements. The relationship between the dog and the human changes, as the hunter gives instructions to the dog based on the location information he is receiving. However, the study points out the need for user-centered design when building technology for human-animal interaction and it also advocates for ensuring animal welfare in the design process.

ACI principles have also been used to improve the task carried out by Diabetes Alert Dogs (DAD) [279, 280, 282]. A DAD is a dog trained to detect changes in blood sugar levels in real-time. These dogs are paired with a human suffering from diabetes, and alert the human when their sugar levels decrease rapidly. However, if the human falls into a coma due to a hypoglycemic attack, the dog is unable to help him. This work proposes several dog-oriented interfaces which could allow the dog to alert emergency services if a critical situation arises (see Fig. 3.5). The task of cancer detection dogs can also be improved by using animal-centered interfaces such as the one described in [180]. Dogs can be trained to recognize several odors from cancer cells using biological samples from the patient. When the dogs find 


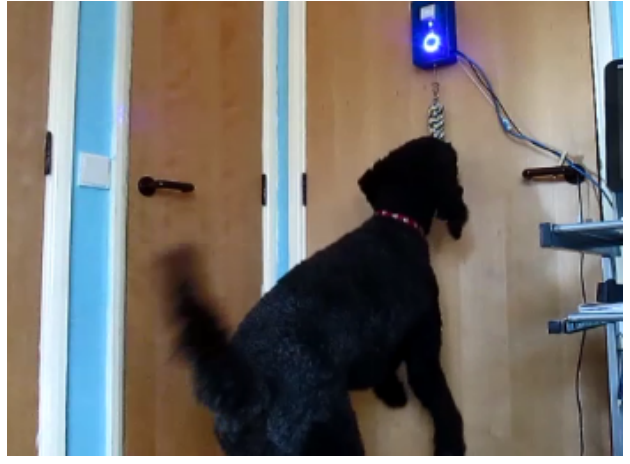

Figure 3.5: Diabetes Alert Dog using a prototype of the alert device used to communicate with emergency services (Image courtesy of Dr. Clara Mancini and Charlotte Robinson)

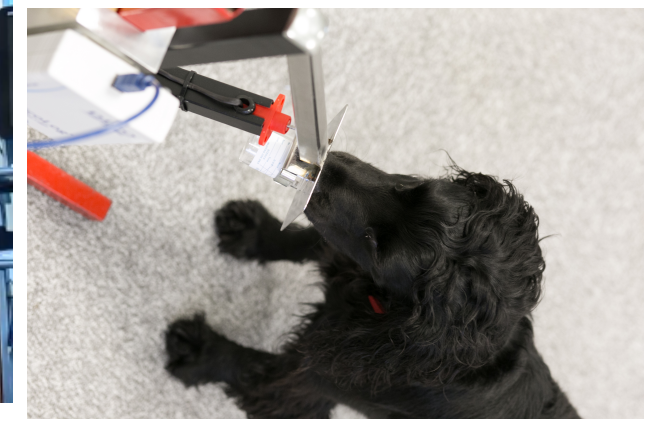

Figure 3.6: Dog using the cancer detection interface (Image courtesy of Dr. Clara Mancini)

a positive sample, they report it to their trainers by performing a specific signal convention. However, sometimes a dog's reaction to a sample is uncertain or spontaneous, and the dogs have no method to indicate the degree of certainty on ambiguous samples. This project proposes a canine-centered interface which allows the dogs to sniff normally on a plate placed over the sample, as they usually do. Using a pressure sensor, the system captures and records the pressure the dog puts on the plate containing the sample (see Fig. 3.6). Each kind of sample causes the dog to sniff with a specific pattern, i.e. the time spent sniffing the sample and the pressure applied on the plate. As a result, the pressure pattern extracted from the sensor allowed more natural and reliable responses from the dogs. Both the project of Diabetes Alert Dogs and cancer detection dogs demonstrate how animalcentered interfaces can not only improve animals' interaction and wellbeing, but also save human lives by enhancing interspecies communication.

\subsubsection{Playful Experiences within Animal Computer Interaction}

The motivational factors which bring animals to play have been the focus of several dissertations $[21,36]$. Although there is no universal answer to the reason why animals play, several works within the ACI research field believe that playfulbased interactions with animals should bring better results in terms of engagement, communication and user satisfaction [116, 254].

There have been several studies where play is used as the fundamental tool to stimulate animals to participate in the activity and interact with the system voluntarily. The main goal of these studies is to improve animals' welfare by 
addressing different issues that can affect the animals' quality of live: sedentary lifestyle, anxiety/stress, routine and boring training exercises, etc.

Several studies have attempted to motivate physical activity among pets using playful devices which cause the animal to move and perform some physical exercise. Feline Fun Park ${ }^{5}$ is one of the tangible playful interfaces which promotes pet activity. It consists of three sensors which monitor the pet's activity level. Depending on the activity level of the cat, the system has three mechanisms to motivate the animal to play at different levels of intensity: two mouse toys and tracer lights. The pet owner is also notified about the cat's activity and he can activate remotely the different mechanisms of the system to encourage playing. However, the playful mechanisms provided are not changing with time, possibly causing that the cat loses interest and stops playing, even if the system continues triggering actions.

Pawsabilities [182] presents a HUI (Human User Interface) and a DUI (Dog User Interface) to reduce canine pets boredom when their owners are not at home. When the system detects that the dog is becoming bored (e.g. by lying on its bed), the HUI notifies the owners remotely so they can activate a mechanism to throw a ball for the dog to play with. On the other hand, whenever social activity is detected on the human side of the system, the DUI activates the video streaming, showing the owners' activity to entertain the dog. This system has not yet been evaluated with enough dogs in order to extract solid conclusions about its benefits to the canines.

LonelyDog@Home [129] is a web based interface allowing humans to interact with their dogs whenever they are away from home. Through a web interface, humans can have a look at their pets, feed them and engage into remote playful activities with them. This work mostly focuses on reducing owner's worries about their pets' wellbeing when they are left alone at home. Pet owners can connect to the system located at their home using any web browser and communicate with their pets using an action oriented interface such as the one shown in Fig. 3.7. On the dog's side there is a ball thrower and an electronic feeder connected to the system, speakers and a webcam. Pet owners can issue pre-recorded audio commands, throw a ball, give the dog a treat or feed him. Although some efforts have been done on the animal's interface in order to provide suitable mechanisms for the dog to interact with the system, there are still some issues regarding the suitability of verbal interactions and visual communication. Dogs' hearing frequencies are different and more acute than ours, thus excellent quality of the audio system is required. Regarding visual communication, LonelyDog@Home allows pet owners to see their dogs, but dogs are not provided with a way of communicating with their owners. Therefore, benefits on animal welfare and anxiety reduction should be further studied for this system.

\footnotetext{
${ }^{5}$ Feline Fun Park: https://www.youtube.com/watch?v=HB5LsSYkhCc
} 


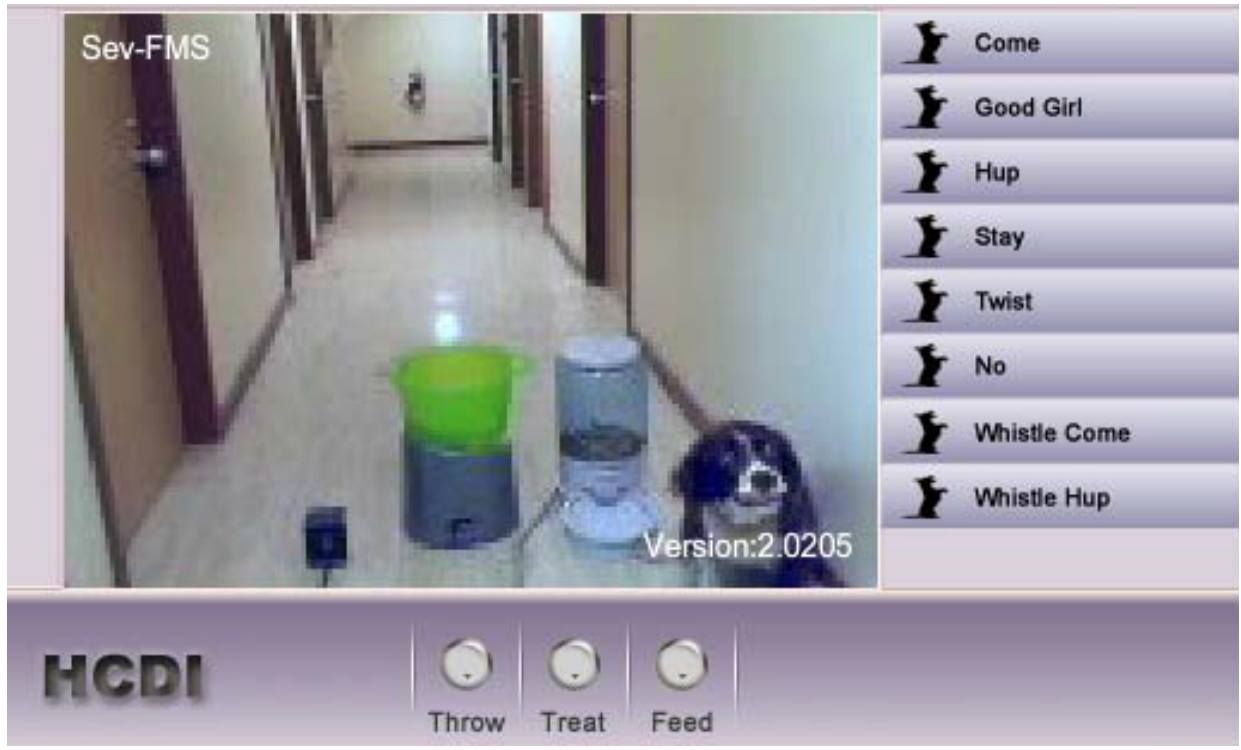

Figure 3.7: LonelyDog@Home graphical interface for pet owners (Image courtesy of LonelyDog@Home's authors)

Other works such as Canine Amusement and Training [359] use play as a mechanism to help both the human and the dog to spend more time together while introducing dogs into training. It offers several kinds of games focused on calmness, obedience and joy. In each game, lights and figures are projected on the ground, and the human is required to give appropriate commands to the dog, which vary in line with the goal of the game, e.g. obedience games require the dog to remain quiet next to the human. In this way, the dog learns how to obey commands in a way that is amusing for both participants. This work allows the human to spend more time with his dog, strengthening their relationship, while providing guidance in a complex task such as dog training. The game has been designed with the assistance of a canine trainer, and the sensing infrastructure has been prototyped with dogs of different sizes.

There are some other systems designed just for the fun of playing and competing. Cat Cat Revolution [224] is a digital game for iPad which shows an animated mouse moving across the screen. Early prototypes of the game allowed to test several combinations of brightness, size, color and movement of the digital mouse in order to accommodate the interface to cat's visual characteristics. The iPad application combines graphical hints and sounds to incite the cat to capture the mouse. There are two playing modes: the digital mouse is moved randomly across the display, or is controlled by a human. In the latter case, the human user connects its iPhone 
to the iPad application, and the screen on the iPad is replicated on the phone. In this way, the human can control the mouse's orientation and velocity by using his fingers. Observational findings derived from a study with 7 couples of cats and their owners showed that the humans considered the game as fun and useful to reinforce their relationships, as well as to create new forms of communication with the animal.

Metazoa Ludens [44] proposes a mixed reality game where a human and a hamster can play together. The playful interface for the hamster is a physical moldable surface which adapts its shape using mechanical actuators. The hamster can enter and exit the playground freely. The human interface consists of a virtual 3D game where two avatars are represented, one for the user and another one for the hamster. The human can move its own avatar through the virtual terrain, and these movements are transferred to a physical bait in the hamster's playground. The real movements of the hamster are also captured and imitated by the hamster's avatar in the digital game. Therefore, a chase between the hamster and the human occurs both in the digital and in the real world simultaneously.

The Playing with Pigs project [5] is an innovative interspecies game designed to strengthen relations between humans and pigs as companions. The pigs are situated in front of a large touch sensitive display showing a light ball controlled by a human player through an iPad application. The iPad application shows the virtual replica of the light ball and the pigs' snouts when they approach the ball. The user has to keep the pigs in contact with the ball and lead them through a triangular target on the screen to score points. However, although this game may be interesting for humans, as they have a scoring scale and goals to meet, it is questionable how much time will pigs pay attention to the game or how could this benefit pigs if they are not aware of the human who is playing with them.

Felino [351, 352] is an interspecies video game designed using ACI principles. The design and development of the game is informed with the animals' experiences and observational feedback gathered from cats' human companions and annotated video recorded sessions. The game allows a human and a cat to play together on a shared tablet screen (see Fig. 3.8). Cats can catch fish and other sea creatures which appear and move across the screen, while humans can control several options of the game, like the size, speed and movements of the creatures. Moreover, every time the cat catches a fish, a sphere is released. Those spheres can be caught by a crab avatar which is always on the screen. The crab is controlled by the human player, and by collecting spheres, new crabs appear following the older ones. Cats can also interact with the trail of crabs the human creates. Therefore, human and cat can cooperate in a shared digital world, and the human can adapt the game to the cat's reactions and preferences.

Although all these projects are based on playful activities, each one has been specifically designed for its own purpose. Moreover, these systems do not adapt 


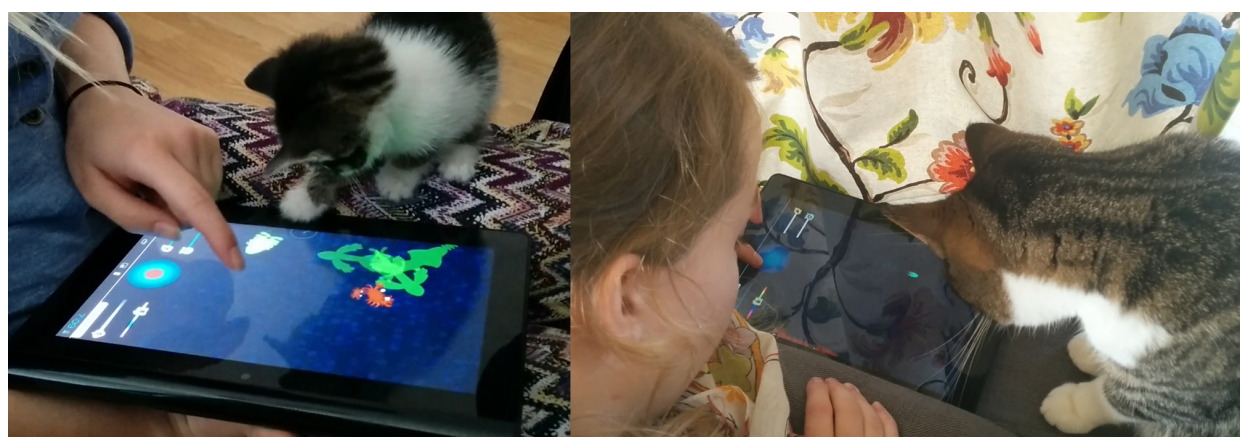

Figure 3.8: Cats playing with Felino (Images courtesy of Michelle Westerlaken)

automatically to changes and in most cases the activity has to be started off by a human. If the ACI community wants to take a step forward in developing natural systems for animals, intelligence, automation and reactivity have to be present in playful environments in the future. In the same way as Ambient Intelligent systems adapt themselves to their inhabitants, by recognizing and anticipating their needs, intelligent playful environments for animals must learn animals' behavior and preferences in order to be able to react properly. A playful environment with these features could automatically create and adapt play activities to engage the animals in physical exercise, raise their mood or train them while having fun. The next section will give a definition for future intelligent playful environments for animals and the features these systems should include.

\subsection{Situating Intelligent Playful Environments}

This work sets the foundation for intelligent playful environments for animals starting with a definition of what they are:

An intelligent playful environment for animals, or IPE4A, is an animal-centered ecosystem with intelligent capabilities which is able to learn from the animals' behaviors and interactions, using the acquired knowledge to adapt itself to the context, creating engaging playful activities which do not necessarily need human mediation to evolve.

In order to provide a conceptual taxonomic framework for the future construction of these environments, their requirements are listed as follows:

- Playfulness. The environment has to consider play as the conductive engine of any activity it creates. 
- Intelligence. The environment must be able to capture and analyze the occupants' interactions and behaviors, extracting patterns and preferences. This knowledge will be useful for the creation and evolution of playful activities, whose purpose and dynamics will be adapted to the context.

- Reactivity and interaction. The system must react suitably to the animals' interactions, and also provide proactive stimuli to the animals to foster communications between the system and the users (both human and nonhuman).

- Animal-centered design. Every intelligent playful environment must be designed and developed specifically for animals, with appropriate devices and interaction methods and prioritizing the animals' comfort, safety and wellbeing.

There are also several features that can vary from one playful scenario to another and should be considered in the design of future IPE4As:

- Number of participants (single-player, n-player $\&$ multiplayer). The playful environment can be designed for one participant (single-player), a fixed number (n-player) or it can respond to any of the participants that walk into the ecosystem (multiplayer). If more than one participant is considered, the design of the environment should include ways to handle abandoning scenarios, i.e. when one or more players leave the game or physically come out of the ecosystem.

- Species of the participants (one species vs. multiple species). Animals probably do not perceive their environment in the same way humans do [189]. Moreover, different animal species may not have the same conceptual view of the world. As a consequence, animals from distinct species will not behave similarly given the same scenario. This affects several design decisions in the construction of interfaces and interactive systems targeted at animals: from the way in which they will be encouraged to play to the reference health values the system will use to create a physical activity. Consequently, the intelligent playful environment can be designed specifically for a single animal species or it can be designed to recognize the animal's species and adapt itself to it.

- Human participation (participant vs. non-participant). Humans may or may not take part in the playful activity. In the former case, the system will only react to animal interaction. In the latter case, it will respond to both human and non-human actions.

- Human presence (physical vs. virtual). If humans take part in the playful experience they can either be physically present in the environment or participate remotely. The remote participation may encompass a wide range of 
scenarios: from pet owners in their spare time at work, to child patients in hospitals seeking amusement and distraction.

- Control. The intelligent features and reasoning engine of the playful environment can learn and take decisions autonomously, i.e. without human intervention, or they can be guided by explicit human knowledge. The latter idea implies that IPE4As can provide mechanisms to allow human users to define explicit behavioral patterns the system must follow. For example, if a zoo worker wants the activity to be paused every day at midday to feed the animals and resumed after all the animals have finished, she should be able to easily program the system with such desired behavior.

- Information acquisition. The system inputs can be gathered by different technologies: wearable devices, sensing (motion sensors, pressure sensors, etc.), video and audio recordings, etc. In all cases, the selected capturing devices should be non-obtrusive and ensure the animals' safety and comfort.

- Learning inputs. Both humans and animals can coexist within the playful environment, interacting with the system and with each other. The design phase of the environment has to establish which of these interactions will serve as learning inputs for the intelligent system. It also has to be decided if only animal interactions will be included or if human inputs will also be considered. In some cases, human interactions with their pets could provide very valuable information to the learning system. As an example, pets are not able to verbally communicate when they are bored, but their owners can recognize their mood and start playing with them. The system could therefore learn which activity raises the pet's mood by looking at the owners interactions with the animal.

- Sense-guided stimuli. Since distinct species may behave differently in the same context, their preferences and motivations may also differ. Some species might therefore feel more attracted by visual stimuli such as lights or mobile mechanisms (e.g. cats), while others would respond more eagerly to olfactory clues (e.g. dogs). In order to use the proper actuators and devices to capture the animal's attention, IPE4As should rely on the most suitable stimuli for each animal species in a given context.

- Single-purpose vs. multi-purpose activities. Playful activities created by the environment can be focused on solving just one issue of animal well-being, e.g. a game which only fosters physical activity. On the other hand, more complete activities covering several issues can also be created, e.g. a game which includes a training element at the same time as physical activity is being monitored and fostered by the system. 
Table 3.1: List of requirements and features of intelligent playful environments

\begin{tabular}{ll}
\hline Requirements & Features \\
\hline Playfulness & Number of participants \\
Intelligence & Species of the participants \\
Reactivity and interaction & Human participation \\
Animal-centered design & Human presence \\
& Control \\
& Information acquisition \\
& Learning inputs \\
& Sense-guided stimuli \\
& Single-purpose vs. multi-purpose activities \\
\hline
\end{tabular}

\subsection{Situating Current Playful Environments for Animals}

The design and development of future intelligent playful environments comprises many factors that should be analyzed and informed by the existing digital games involving animals. Table 3.2 shows a classification of the existing digital playful experiences for animals described in Section 3.2 in terms of the game features outlined in Section 3.3. The next subsections will open the discussion about where should intelligent playful environments put their efforts to improve current lacks in playful scenarios, and how could ACI research inform the design of future intelligent systems for animals.

\subsubsection{Game Participants: Static or Dynamic Approach?}

Human participation is considered important if we want to strengthen the relationship between humans and other species. Nevertheless, some works have left open the possibility of the human joining the game, allowing the animal to participate alone if the human is not available. This should be an important requirement if the animal is going to spend considerable time alone or separated from the human.

In games requiring human participation, two tendencies have been detected. The philosophy behind games such as Pawsabilities and LonelyDog@Home only makes sense when the human is distant from the animal, and thus remote communication is the only way of human interaction with the system. Other works such as MetazoaLudens or Pig Chase can take place either with humans physically present in the same environment or with them remotely interacting with the interface provided. In order to reach a higher degree of flexibility, we propose that intelligent playful environments support both animals playing alone and together with their human companions, the latter case with its two modalities: remote or 


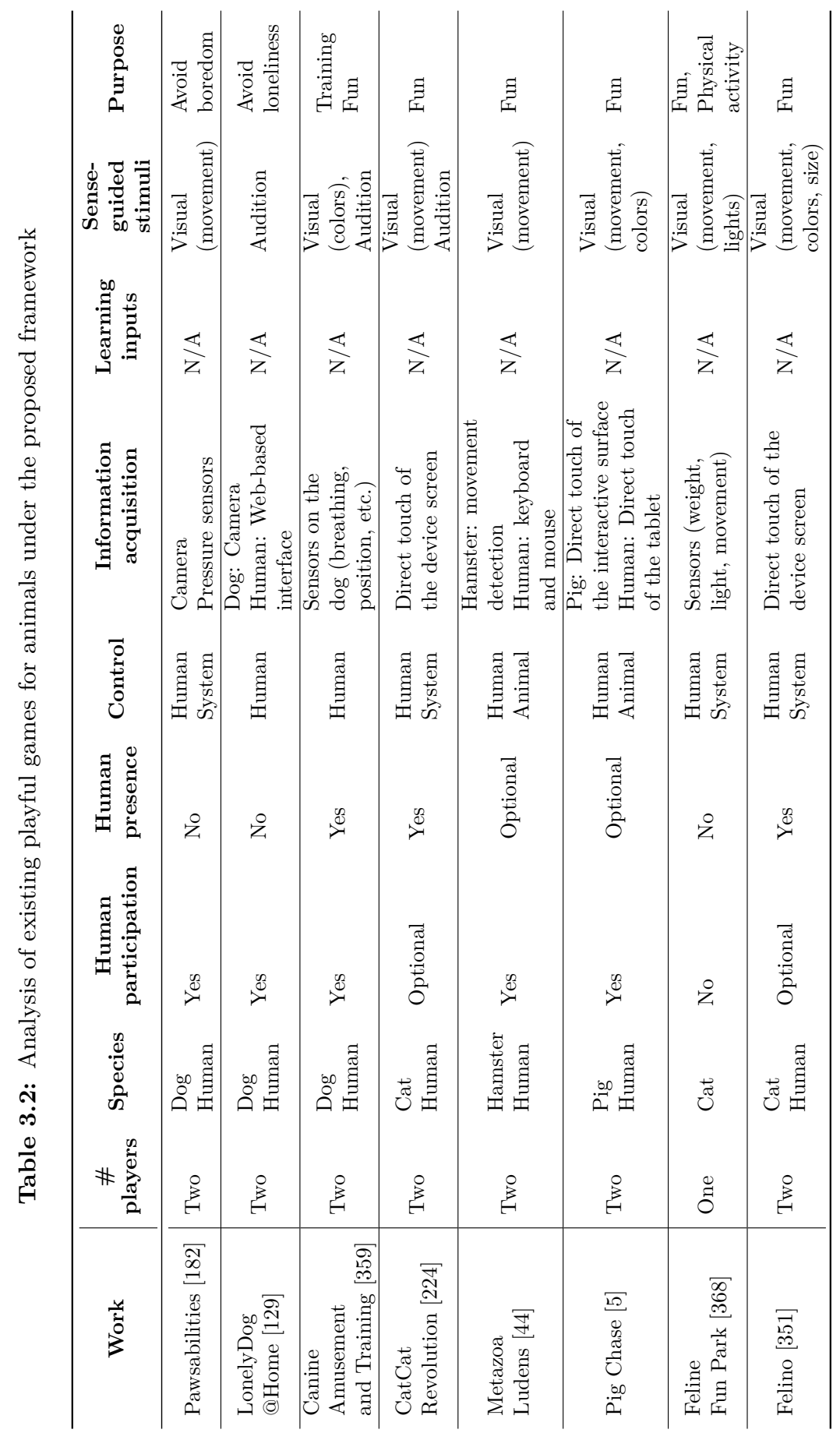


in-person participation. The environment should adapt the game to the context of the moment, allowing the human entering and exiting the game at any time without causing frustration to the animal. For example, if a human is playing with her dog but suddenly a phone call interferes, the human should be able to answer the phone without causing the game to terminate. The game should be adapted to continue without the human player, and if eventually the human wants to get back into the game, the system should create the appropriate game flow in order to incorporate the human back into the playful activity.

The same argumentation can be applied to animal participants. The feature number of participants in Table 3.2 indicates the number of players the game was originally designed for. As an example, it is understood that several cats could be playing simultaneously to chase a mouse on the screen of CatCatRevolution. However, the system does not distinguish between the touch of different cats on the same screen and thus, to the system's knowledge, there is only one cat playing at a time. It can be seen that only games for one or two players have been designed, and two player games always include a human participant. A more dynamic approach should be provided in future intelligent gaming environments, where several animals and/or humans could participate. The participation of an animal/human in the game implies that the system recognizes him as a new and differentiated user from the other participants of the same species. Therefore, both animals and humans should be able to enter and leave the game whenever they need to. Neither the human nor the animal should become deprived for their decisions about participating or not in the game. The game should be adapted to the number of current participants, starting when the first participant comes in, and terminating when the last participant abandons the game.

Until now, humans are the agents mediating the interaction between animals and computing interfaces. From the eight games being analyzed, six of them require the human to start the playful activity. Only two of them can autonomously perform some interaction to attract the animals' attention, and both of them monitor the animals' activity level in order to notify the human in case they want to intervene. It is essential for the future development of intelligent playful environments that the system itself could decide to initiate or terminate a playful experience. Firstly, if the system detects some need on the animals and there are no humans around, the environment should be able to start the playful interaction in the same way a human would do when detecting some animal's urge. Secondly, some animals may want to play the whole day, but it might be inadequate because of health and behavioral reasons. The system should be able to end the playful activity when it detects that the purpose of the activity has been met. In this context, there are several questions that need to be previously addressed:

- How can the animal be aware that the system wants to initiate the interaction? 
- How can the system involve voluntarily the animal into the playful activity?

- How can the system itself communicate or attract the animal in order to start a playful experience?

- How to end the playful activity without negatively affecting the animal?

- How to make the animal understand that the playful activity has ended?

Another important issue that has not been addressed yet is the possibility of the animals initiating the playful experience. How can we build successful playful experiences for animals if we do not allow the animal to start playing freely at their own will? Several questions arise around these ideas, and further studies within the ACI field should bring new insights on how to provide the best suitable way to let the animals decide when to play:

- How can animals initially learn that the system will respond to their actions by starting a playful experience?

- Which mechanisms/behaviors will animals use to start the interaction with the system? Will they use the same behaviors they use to communicate playful intentions with humans/other animals?

- How can the animal withdraw from the playful experience?

- How can the system recognize that the animal wants to stop playing in order to stop all the interaction?

- Could the system analyze the factors which lead to the end of the activity and use this information to improve the next playful experience, by making it more appealing and time lasting?

\subsubsection{Adapting Computer Interfaces to a Broader Audience: Species Awareness and Interrelationships}

Regarding the species of the animals' participants, it is observed that most of the games have been designed for dogs or cats, while only one game has been developed for small pets such as hamsters. It is remarkable that only one of these games has considered animals outside the pets' domain as active players, which gives an idea of what kind of users ACI research is currently addressing. Perhaps pet companions are the first animals coming to our minds when we think about the animal kingdom, but we shall not forget to address other animal species that may also require playful environments. Wild animals could also benefit from ACI advances: if computer mediated interaction can help us to communicate with wild species by means of play, our knowledge about them will improve significantly. 
Moreover, semi-wild species such as animals living in zoos could also benefit from playful interactive environments, as it will be described in Section 3.5.

Another issue to be solved is that current digital games for animals only address one animal species at a time. Interspecies relationships between animals, although frequent in natural environments, are not supported by current playful interfaces. An intelligent playful environment for animals should support this variability and foster interspecies relationships, creating suitable games for different animal species playing together. This is a challenging requirement, as different species understand their surroundings in a different way and react differently in front of the same situation. The design of this kind of games should be informed by previously studying the relationships and playful dynamics of the involved species. Nonetheless, there might exist some cases where the playful interaction cannot be performed due to several reasons: physiological incompatibility of the animals, opposed behavioral reactions, etc.

Despite the difficulties introduced by species variability, ACI studies should take advantage of these differences when it comes to perception and motivational factors. Existing playful games have already tried to appeal to the animals' sensing acuity, capturing their attention with visual clues like moving objects, audio commands or sounds. However, it remains to be studied the effects of different types of stimuli in the animals' attention regarding its species, in order to give a detailed classification which could inform the development of future engaging playful scenarios. Some questions to be addressed are:

- Which is the most appropriate mechanism to start the interaction with the playful environment for a specific animal species? How can this mechanism vary among species?

- Which stimuli are more adequate for each animal species in order to capture and maintain the animal's attention during the game?

- How can animals be motivated to perform some specific activities/tasks during the game? How are these motivational factors influenced by the animal's species?

\subsubsection{Broadening the Horizon: More Devices, More Fun!}

The reported games rely on a single electronic device to interact with the animal. Only Feline Fun Park and LonelyDog@Home introduce more than one device to entertain the animal, but still there is no communication between the different devices being used, nor a coherent relation between them. Animals playing with the same device over and over again are likely to become bored or lose interest 
when the novelty factor vanishes. The same could happen eventually with several unrelated devices in the same environment.

An intelligent playful environment should be comprised of not only several and diverse devices, but also interconnected and meaningful. The devices conforming the intelligent environment should be able to cooperate and communicate with the system and the other devices, in order to create elaborated activities which can vary from one iteration to the next one. As an illustrative example, we could think of an intelligent playful environment including several electronically controlled balls, a flying drone with a camera, and an electronic pet feeder. The goal of the interactive game would be to teach sheep-dogs to bring the flock to their masters and learn commands that are commonly used in this task. In this case, the electronic balls would represent the flock and would move according to the behavior that needs to be taught. A sound system would reproduce voice commands and the drone with a mounted camera would track the behavior of the sheep-dog by using computer-vision algorithms. If the dog would not act as previously trained, the system would notify this situation so that further training would be later performed with the presence of a human master. However, if the sheep-dog reacted as expected a reward would be given by the automated feed machine. Having several interactive balls would allow the simulation of different real situations that may occur with real flock that needs to be kept under control. The flying drone would also control the position of the electronic running balls so that they move in a challenging way depending on the capabilities of the dog being trained. The coordination of several devices in this scenario would allow the autonomous training of sheep-dogs when master trainers may not be present.

The final goal/s of the activity will help to identify which kind of devices would make sense together. The system should learn how to better connect and join together the different individual devices, and how to evolve the game when required.

\subsubsection{Decision Making and Adaptation: Who Controls the Controllers?}

Although some of the aforementioned games allow the human user to modify several options such as movement direction of the objects, releasing treats, etc., these are just straightforward ad hoc configurations. When the human does not intervene, the system can run the game with the default configuration without any major concern. However, having multiple interconnected devices will significantly increase the configuration possibilities, and the human user will not always be participating in the playful experience to control or guide the decisions of the game. As a consequence, the system should intelligently manage the resources and take control of the decisions, adapting the game to the context and the current players, such as in the sheep-dog example in Section 3.4.3, where the electronic balls adapt their movement to the command to be practiced. 
Context-awareness and adaptation should be performed in the same way as AmI scenarios adapt themselves to human users: by extracting knowledge from the users' interactions with the system. None of the presented games in Table 3.2 apply any type of reinforcement learning from the inputs of the system. The construction of future intelligent playful environments should consider these interactions as essential inputs for the learning subsystem.

Nevertheless, not all the responsibility of the game creation should rely on the learning capabilities of the system. There are many situations where the system may not have the best information to take a decision. Moreover, not all the possible scenarios can be controlled or anticipated. Specially, external knowledge from the human users could be essential in the first attempts of the environment to create a new game, when the learning algorithm still has no information. Hence, human users should also be provided with an adequate way of participating in the decisions beside the need for learning algorithms to implement context-awareness in playful environments. Human users without programming experience should be able to manage the environment and define explicit behavior to inform ambiguous decisions, or specify particular scenarios. HCI techniques and studies have already been applied in order to come up with easy-to-use and useful interfaces to allow the definition of explicit behavior by end-users $[42,83,186]$. The same philosophy could be applied to bring intelligent playful environments with explicit knowledge from the human participants.

\subsection{Application Scenarios for IPE4A}

Considering the described requirements and features that intelligent playful environments for animals should accomplish, and after studying the lacks and limitations of existing approaches, the scenarios in which these systems can be deployed have been analyzed and the benefits they can provide in different domains are presented here.

\subsubsection{Mental Well-being}

Not only humans but also animals need to socialize. However, domestic pets spend most of their day alone at home without interacting with their human friends. Even when the human is at home they may not receive all the affection they need. Similarly, zoo animals live inside a restricted ecosystem, sometimes being the only one of their kind and without being able to interact with humans on the other side of the glass in any way. Another risk group are animals living in shelters [176], where volunteers are unable to give all the animals the attention they require due to lack of resources and people. All these animals can suffer from isolation, sadness and anxiety [6, 295, 296], far from achieving a fully happy existence. An intelligent playful environment could detect whether an animal is becoming bored 
or stressed, and study the best way and best moment to create fun activities to stimulate and entertain him and keep his mind active. For this purpose, the intelligent environment should have previously learned the animal's favorite games and interactions and the most effective sense clues to gain his attention. However, these kind of playful activities, the moment when they are conducted and the consequences on the animal's well-being should be studied in depth in order to avoid behavioral problems or causing stress.

\subsubsection{Physical Activity}

Another crucial element to enhance animal well-being is physical activity, which has to be stimulated in cases such as the ones described above when the animals do not receive all the required attention for long periods of time. When an animal does not receive any external stimuli or is feeling depressed, it would not feel like initiating physical exercise. In this case, the environment could capture the animal's attention and engage it in playful activities to make it move and perform some physical exercise. The system could adapt the exercise to the animal's physical attributes and habits in order to create a healthy and amusing routine. Other variables to be taken into consideration should be the frequency, duration and time when the activity should take place. The potential improvements the environment could bring on animals' welfare should be studied considering the aforementioned factors in Section 3.3.

\subsubsection{Training}

Playful environments can also be an enjoyable way of fostering training activities without overloading the animal with strict orders. Tough training and repetitive activities can cause loss of attention and refusal to participate. By transforming the learning activity into a game, it would not be presented as a mandatory and strict activity, and animals might be more inclined to participate. Using playful activities for training could also alleviate the animals' stress and sense of responsibility derived from such a demanding task.

The design of intelligent playful environments for training scenarios should be carried out with the guidance of a professional trainer. Intelligent environments for animals should allow playful training with or without the presence of a human. In case of pet owners, not skilled in training activities, the environment could help them to perform successful practices. The owner's participation in the activity could also reinforce his bonds with the animal. However, some animals will not have the opportunity to be trained by playing with a human, such as in shelters where few volunteers have to attend hundreds of animals. The environment should then be responsible of teaching new behaviors to the animals, adapting the training to their learning pace and motivation. 


\subsubsection{Therapy}

Animals can help in the rehabilitation of people recovering from illnesses or disabilities [71, 146]. Interactions with animals can reduce patients' anxiety [12] or help children with autism in socializing tasks [307]. In the digital era where we live, some rehabilitation tasks rely on computer-based technology [166]. Understanding animals' interactions with computer-based systems could help to introduce animals within these therapeutic activities, e.g., incorporating animals in the context of rehabilitation tasks for people with disabilities such as brain acquired injuries, or creating playful health oriented activities with animals for elder people.

In situations where the animal cannot be physically present with the subject the playful environment could serve as a bridge to bring the patients closer to the animals. Patients could remotely interact with the system via a human-computer interface, by activating devices in the environment or responding to the animals' interactions. As a consequence, some sort of non-verbal communication could emerge between humans and physically distant animals, originating an enriching experience for both sides.

\subsection{Challenges and Considerations}

Developing intelligent systems capable of adapting themselves to the context requires ensuring several safety aspects. The system should not harm the environment nor the users in any possible way. This is of special relevance when users cannot be taught how to use the system, and thus, free interactions and behaviors are allowed. Therefore, the system should respond to the predefined interactions only. Unexpected behavior must not trigger any reaction of the system.

As has been previously defined, playful systems could allow the animals to play without human supervision. It implies that the animals could be the ones who decide when to start the game, or end it. However, animals may not be conscious about the emotional or physical effort the activity is demanding from them. For example, if a dog is playing a throw-and-catch ball game, which they usually love, it will not stop demanding another round unless it gets exhausted. This physical fatigue may eventually become dangerous if it happens repeatedly. The system should control the animals' physical activity in order to avoid exceeding the limits of what a healthy exercise should be.

Another potential pitfall when allowing the animals to play without human supervision is the material damages that they can unintentionally cause in their environment. The game should be conducted within a safe area where physical objects, such as furniture or electrical amenities, do not interfere in the activity. Otherwise, the animals may collide with these elements, injuring themselves or damaging them. For these reasons, the system or the human should define the 
physical boundaries of the playing area. The devices involved in the game should be placed within this area, and their operational range, i.e. the area where the animals will interact with the device, will not surpass the defined limits. Potential dangerous objects for the animal should not be placed within this area. Moreover, fragile or valuable objects shall not be placed either in the playing area in order to avoid unwanted consequences.

When addressing animal safety, we are not only considering physical welfare: mental wellbeing should also be guaranteed. Even if the game does not demand hard physical exercise, the animal could get extremely excited because of the joy it is experiencing. Enjoying the playful activity is essential, but the excitement levels should not exceed the limits of what is salutary. Expending long periods of time under these conditions, inadequate playing schedule (such as allowing play when the animal should be sleeping), or even an abrupt termination of the game by the system could led to stress, anxiety and/or overexcitement. Humans are able to handle these undesired feelings, calming themselves down and returning to a more peaceful state. However, animals may not manifest the same kind of self-control over their emotions and the physical response these emotions trigger. In order to avoid unhealthy mental feelings, the emotional states of the animal should be gathered. The playful environment should detect whether the animal is entering into an undesired emotional state, readapting the activity to take the animal back to a more relaxed situation. Moreover, some limitations should be defined on the schedule and duration of the playful activity, either by the humans or by the system. It will help to create a healthy routine, avoiding bad behaviors derived from inadequate schedules.

The potential of emotion identification is only comparable to the difficulty of conducting such a complex task. Identifying emotional states is a challenging requirement for any kind of system, although there are some successful results concerning human emotion [199, 244]. Within the animal domain, the physical evidences of an emotional state may differ from one species to another. Nevertheless, for each species there might be some physical parameters which could help to identify their emotions. We could classify these parameters into two different categories: observable and measurable. Examples of observable parameters are ear position, body posture or tail movement. The aggressive emotional state of cats is easily identifiable using observable parameters: ears back, open mouth showing teeth and bended body. Regarding measurable parameters, we could refer to the heart rate or the number of times per minute an animal waves its tail. Excitement, for example, is an emotional state which could be better identified using measurable parameters. However, gathering measurable parameters imply the animal has to wear specific devices, which could be obtrusive and interfere with its normal life. In contrast, observable parameters will require using cameras and sophisticated image recognition methods, which could restrict human privacy in shared environments. The identification of emotional or mental states in animals, and 
its use in the adaptation of the playful environment should be carefully studied for each case, analyzing the benefits and trade-offs its deployment could lead to.

The intelligent playful environment must, in all cases, be unobtrusive both for the animals' and humans' lifestyles. The animals' natural behavior must not be biased nor interfered by the devices which form the environment and the mechanisms used to gather information about them. Domestic animals are more used to face new objects and even digital elements in their daily routines. However, wild animals live in natural ecosystems, being unaware of the existence of any digital elements. Similarly, semi-wild animals use to live in either delimitated areas, like farms, or in artificial spaces which reproduce their real ecosystems, like zoos. Semi-wild animals may be used to human presence or even cameras, but the interaction between them and the digital world is limited, if not inexistent. If any technology is intended to be used within these environmental conditions, the animal must not perceive it as a potential danger. One way could be introducing the different elements conforming the playful environment gradually, i.e. one at a time and introducing the next element once the animal has become used to the previous one.

\subsection{Conclusions and Future Work}

This work proposes a new line of research in the recently emerged field of Animal Computer Interaction: intelligent playful environments for animals. These environments will ground on the most inherent behavior of animals: play. Around playfulness, an intelligent environment will generate engaging games for animals. The environment will learn from the animals' interactions, adjusting the game to their needs and requirements. The playful activities created by these environments could help animals to overcome possible issues such as isolation, poor physical condition, repetitive training exercises or remote digital interaction with human-beings. Moreover, we believe that intelligent playful environments for animals would be the perfect scenario in which to study animals' interactions with digital devices, as the animals will engage voluntarily in the playful experience. The benefits derived from IPE4A could apply both to human and animals' wellbeing.

A conceptual taxonomic framework has been laid down for the future design and development of these environments. Existing games based on technology for animals have been analyzed in terms of the proposed framework, detecting some shortcomings that intelligent playful environments could help to resolve. Several applications have been outlined, highlighting the benefits of applying intelligent playfulness to animals' interactions with digital ecosystems. 
Future work essential for the successful construction of IPE4As includes the definition of a formal development methodology covering the aforementioned features and requirements. Each of these features should be carefully studied in order to determine how they will affect the construction of the environment and the users' well-being, and whether they should eventually be taken into consideration in the development process regarding the specific circumstances.

The first step for the design of intelligent playful environments should be studying the most fundamental game phases, which will be common in a range of playful experiences that could be created. Considering the playful activity as a story/performance in which the actors will be the animals, the most basic and common phases in which we can decompose such stories will be the introduction, development and conclusion. Therefore, the most fundamental interactions within an intelligent playful environment will be the initiation of the activity (introduction), the transition from one stage/goal to another (development) and the termination of the game (conclusion). A set of experiments is being designed to study these three game phases that every playful experience contains. These experiments aim to answer some of the questions raised in Section 3.4.1: how could the environment gain the animals' attention and whether animals would be willing to initiate the playful interaction. These experiments will also study how different types of stimuli affect the animals' engagement in each of the three aforementioned game phases. For this purpose, we will evaluate the animals' reaction to smell, sounds, lights and moving devices in order to find the most suitable interaction for each context.

In addition, we are defining in our on-going work a flexible intelligent behaviormanagement system for reactive environments. It will learn from the users' habits and preferences, extracting behavioral rules. The human end-users of the system will also be able to define their own personal behavioral rules and incorporate them into the environment. The behavior-management system will therefore combine two ways to incorporate behavior based on automatically acquired knowledge and explicit knowledge specified by humans. This powerful combination will allow the development of playful environments able to adjust to a wide range of situations more effectively, without having to develop a specific system for each scenario.

\section{Acknowledgments}

This work was partially funded by the Spanish Ministry of Science and Innovation under the National R\&D\&I Program within the project CreateWorlds (TIN201020488) and from Universitat Politècnica de València under Project UPV-FE-201424. It also received support from a postdoctoral fellowship within the VALi+d Program of the Conselleria d'Educació, Cultura I Esport (Generalitat Valenciana) awarded to Alejandro Catalá (APOSTD/2013/013). The work of Patricia Pons 
has been supported by the Universitat Politècnica de València under the "Beca de Excelencia" program, and currently by an FPU fellowship from the Spanish Ministry of Education, Culture and Sports (FPU13/03831). 


\section{Part II}

\section{Study and Recognition of Playful Interactions}



After the definition of what a playful interactive environment is, this part of the thesis aims to explore how to support the design and development of these interactive environments for animals following an animal-centered approach and allowing for the animals' spontaneous interactions during the technologically mediated playful activity. For this purpose, the following chapters will explore the use of Natural User Interfaces (NUIs) for animals and the creation of a system based on NUIs to support the design and development of suitable interactive spaces for them.

The first step will be to analyze how to create a system capable of detecting animals' spontaneous behavior, recognizing their interactions and types of behavior during the game, and adapting the playful experience to them. This exploration of the use of NUIs for animals led to the design and development of a non-wearable depth-based tracking system to detect a cat's body posture and body parts in real time in a playful scenario. The initial evaluation of the depth-based tracking system is described, followed by an exhaustive evaluation of the performance of several classification algorithms based on both a supervised and a knowledge-based approach.

In parallel to the design and evaluation of the non-wearable tracking system, this part of the thesis describes the first step in studying how NUIs could be adapted to create interactive spaces for different animal species, with an evaluation of domestic animals' preferences in relation to technological stimuli.

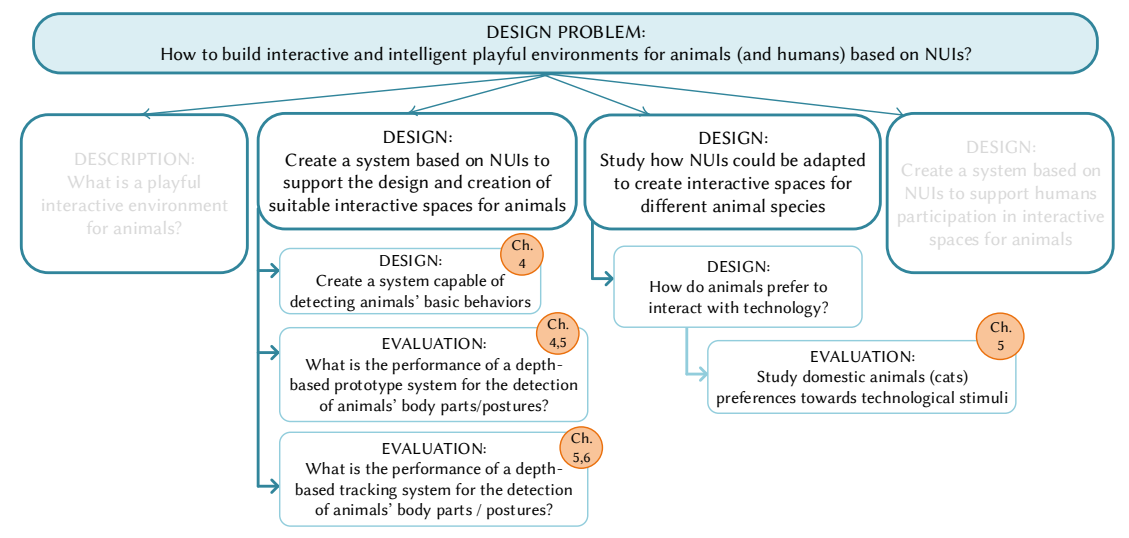

Figure 3.9: Problem decomposition of the work described in Part II. 



\title{
Chapter 4
}

\section{Developing a depth-based tracking system for interactive playful environments with animals}

\author{
Patricia Pons ${ }^{1}$, Javier Jaen ${ }^{1}$, Alejandro Catala ${ }^{2}$ \\ ${ }^{1}$ ISSI Group, Department of Computer Systems and Computation (DSIC), \\ Universitat Politècnica de València, Valencia, Spain \\ ${ }^{2}$ LoUISE Group, Computing Systems Department, \\ University of Castilla-La Mancha, Spain
}

Published in Proceedings of the 12th International Conference on Advances in Computer Entertainment Technology - Second International Congress on Animal Computer Interaction, pp. 59:1-59:8, ACM (2015). doi:10.1145/2832932.2837007

\begin{abstract}
Digital games for animals within Animal Computer Interaction are usually singledevice oriented, however richer interactions could be delivered by considering multimodal environments and expanding the number of technological elements involved. In these playful ecosystems, animals could be either alone or accompanied by human beings, but in both cases the system should react properly to the interactions of all the players, creating more engaging and natural games. Technologically-mediated playful scenarios for animals will therefore require con-
\end{abstract}


textual information about the game participants, such as their location or body posture, in order to suitably adapt the system reactions. This paper presents a depth-based tracking system for cats capable of detecting their location, body posture and field of view. The proposed system could also be extended to locate and detect human gestures and track small robots, becoming a promising component in the creation of intelligent interspecies playful environments.

\subsection{Introduction}

The development of suitable engaging games for animals is a promising research line within the field of Animal Computer Interaction [173, 174]. However, until now, interactive digital games for animals have been tied to a specific device, human participation has usually been limited to a "controller" or "assistant" role and there has been no support for several animal participants playing together as differentiated players [258].

Traditional games with animals rely on a more natural and open interaction, meaning that both animals and humans can move freely during the game. Animals are used to playing by themselves or with humans, and in the latter case the human is an active and essential participant in the activity. In addition, traditional games make use of the elements in the environment to enhance the playful experience, not limiting it to the object itself but to the spontaneous interactions between the players thanks to the mediating object. Moreover, it is not unusual that when a human starts playing with one of his pets other animals in the room join in, forming a multiplayer activity in which the roles of the participants evolve during the game.

Future technologically-mediated games for animals could therefore be conceived as multimodal and multi-device systems, in which animals could play either alone, in a group or with human beings in a natural way. If animals play by themselves, the system should intelligently manage the different devices and objects in the environment in order to adapt the game to the animals' preferences and interactions $[254,258]$. As an example, we could think of a game in which a cat chases a Sphero ${ }^{\circledR}$ (electronic ball) controlled by the system, but the movements of the ball are not random. Instead, the Sphero ${ }^{\circledR}$ could be programmed to move away from the cat inside its field of view, or suddenly turn left or right if the cat gets too near to it, or even move towards the cat if the tracking system detects that he is crouched in a hunting position, waiting for the ball to approach. If human beings are also participating, their interactions should also be considered an essential part of the game and the digital playful experience should be as natural as traditional games. 
The use of non-wearable tracking mechanisms would allow more natural interactions within technologically-mediated environments, not limiting the interaction to a specific device or interaction modality, but instead letting the participants explore their environment and create the dynamics of the game on the go. For this purpose, the tracking system should provide the system with information about the animals' location, body posture and field of view, as well as the location and gestures of the human participants, if applicable. This information would allow the development of playful experiences which improve the animals' wellbeing by introducing new forms of mental and physical stimulation. This paper will describe the development of a prototype for a depth-based tracking system for cats and discuss its future extensions to human gesture recognition and object tracking. Several application scenarios will be outlined, and future work and possible research lines will be given.

\subsection{Related Works}

Outdoor animal tracking systems are either based on GPS or radio-frequency collars only $[179,235,344]$, or they could also use wearable inertial measurement units (IMUs), such as accelerometers and gyroscopes located on a collar [158] or attached to a harness [30, 34]. In the first case, the system only gives information on the animals' location but not about its postures or orientation. In the latter case, classification algorithms are used on the sensors' data to identify the behavior and/or body postures of the animal.

Indoor animal tracking systems usually rely on wearable devices to gather information on the animals' movements. Canine Amusement and Training [359] uses a wearable harness with attached IR emitters to detect the position and posture of the animal. Cat@Log [367] uses a cat collar device with several sensors: a camera, a GPS, an accelerometer, a Bluetooth module, battery and micro SD card. The camera provides videos of the cat's view, while the accelerometer data is used for activity recognition such as sleeping, jumping, walking or scratching. However, it would be difficult to use this activity recognizer to detect specific interactions during a game. Poultry.Internet [164] tracks the movements of a chicken using a camera and an electro-pad located on the chicken's leg to sense its muscle activity. Through camera images they also detect the chicken's head to find the orientation of the animal within the backyard, but no postures are identified. No wearable device is used in Purrfect Crime [323], an interspecies digital game for cats and humans in which a Microsoft Kinect ${ }^{\circledR 1}$ is used to detect the position of the cats inside the play area. However, the system only locates the central position of the animal, introducing some interactions which were not really intended by the cat.

\footnotetext{
${ }^{1}$ https://msdn.microsoft.com/en-us/library/hh855355.aspx
} 
Within Human Computer Interaction, the arrival of the Microsoft Kinect ${ }^{\circledR}$ sensor introduced new and natural interaction modalities, simplifying indoor human tracking and gesture recognition by using depth information of the scene. This sensor is usually placed in front of the humans interacting. However, multimodal playful scenarios for human players frequently place the depth-sensor at a higher position [23, 139, 140], providing wider playing areas and avoiding occlusion due to elements in the room. Technological playful environments for animals would require multimodal interactions inside spacious scenarios. It would be preferable to avoid wearable trackers as they could limit the agility of some animals. Therefore, a depth-based tracking system would be a promising way of detecting the animals' location, posture and field of view, taking the development of intelligent playful environments for animals a step forward.

\subsection{Tracking System}

In order to analyze the suitability of a non-wearable tracking system for animals based on depth information, several sessions with cats were recorded using a Microsoft Kinect ${ }^{\circledR}$ sensor, in which cats were playing with their owners or caretakers, or with small robots. These sessions allowed us to obtain real data on playing postures, behaviors and movements, which were later analyzed and post-processed to develop a preliminary version of the tracking system.

\subsubsection{Set-up}

The set-up for the tracking system consists of a Microsoft Kinect ${ }^{\circledR}$ sensor looking down from the ceiling, at a height of $250 \mathrm{~cm}$. At this height the sensor covers an area of approximately $200 \mathrm{~cm}$ long and $270 \mathrm{~cm}$ wide, as shown in Figure 4.1. The Microsoft Kinect ${ }^{\circledR}$ is capable of recording both color and depth video streams at a rate of 30 frames per second with $640 \times 480$ pixel resolution.

\subsubsection{Cat Tracking}

The Microsoft Kinect ${ }^{\circledR}$ sensor provides both color (see Figure 4.2a) and depth streams (see Figure 4.2b). The first processing step of the algorithm consists of extracting the cat's pixels from the depth frame. Each depth frame provides, for each pixel, the distance in millimeters from the camera plane to the nearest object in that particular pixel (see Figure 4.2b). The height of the sensor may change between sessions, however as it remains the same within a single session the pixels corresponding to the ground can be discarded by adjusting the system during the set-up process (see Figure 4.2c). However, background segmentation methods, such as plane fitting algorithms, should be used in the future in order to provide more flexible set-up conditions. With the floor removed from the image, 


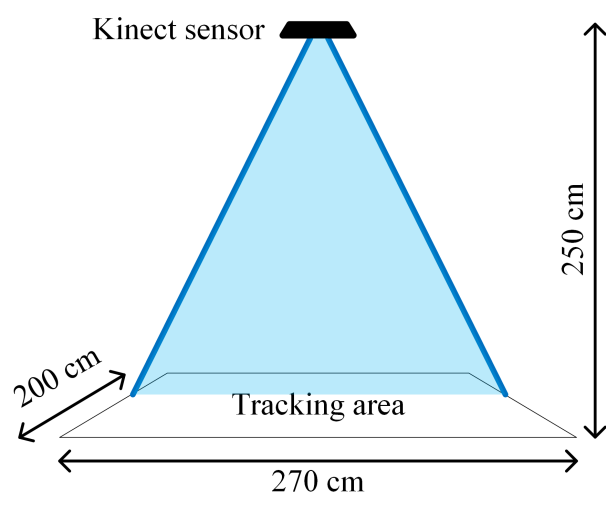

Figure 4.1: Set-up for the tracking system.

computer vision algorithms are applied to extract the cats' contours (see Figure $4.2 \mathrm{~d}$ ). In this step, a cat's location within the tracked area can be determined. To detect the cat's posture and orientation, each detected contour is processed by a k-means clustering algorithm, which groups the pixels by their depth value and relative position (see Figure 4.2e): pixels of similar depth which are located together in the image would be grouped together within the same cluster. The number of clusters was set to three as an initial trade-off between efficiency and accuracy. Further experiments should be conducted to determine the number of clusters which maximizes the trackers' performance. The clusters obtained from each cat's contour are then classified by a customized decision tree algorithm as head, body and tail. Finally, an orientation vector can be defined from the center of the body/tail cluster to the center of the head cluster (see Figure 4.2f), roughly estimating the cat's field of view.
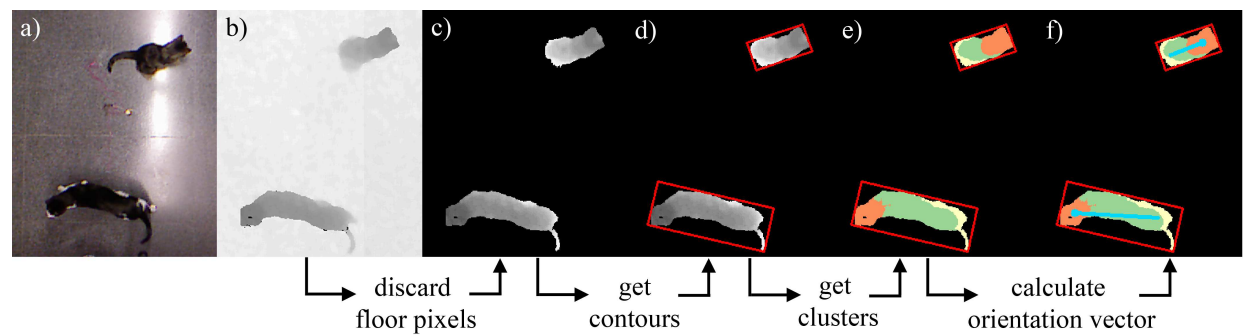

Figure 4.2: Process of extracting the cat's orientation: (a) color frame (b) depth frame, (c) background segmentation, (d) cat contours, (e) clusters for head, body and tail, (f) orientation vector. 
The identification of each cluster depends on the posture being analyzed. Different cat postures were seen to generate different cat contours in the processed depth frames. The cat's depth stream contour when sitting showed a smaller, squareshaped bounding rectangle (see Figure $4.2 \mathrm{~d}$, cat at the top of the image), while the depth stream when standing or walking revealed a larger and rectangular-shaped bounding rectangle (see Figure $4.2 \mathrm{~d}$, cat at the bottom of the image). In addition, the pixels of the sitting cat's head had a significantly higher average depth than the pixels of the rest of the body. This is observed in Figure 4.2d, in which the grey pixels of the head are significantly darker than the pixels of the cat's haunch. In contrast, the cat walking in Figure 4.3 has an average depth of its head pixels very similar to the average depth of the haunch and tail pixels. This can be observed in Figure 4.3b, in which the grey pixels representing depth values are very similar in the whole contour of this cat. In addition, there are some postures, such as the sitting posture in Figure 4.2, in which the tail is not detected because of its proximity to the ground. In this case, the head is clearly differentiated from the rest of the body, and in terms of average depth it is undoubtedly the highest cluster detected. Hence, in this posture only the detection of the head and the body clusters would allow the cat's field of view to be determined. However, in other postures the detection of the tail is crucial to determine the orientation of the cat. For instance, determining whether the cat is looking north or south in Figure 4.3 would be difficult if the tail was not detected, because the head is not clearly in a higher position than the rest of the body. Therefore, our decision tree algorithm considers the following parameters to identify body postures and classify the clusters: area of the cat's contour, number of pixels for each cluster and average depth for each cluster. This preliminary version of the tracking system is already capable of detecting sitting, semi-sitting, walking, standing, jumping and turning positions of several cats at a time, and classifying the different pixel regions in each posture to detect the head, body and tail of each cat. The algorithm runs with live data at a rate of four frames per second on an Intel Core i5 660, without using GPU processing. The performance of the tracking system would be significantly improved by using GPU computing power as well as more efficient implementations of the clustering algorithm.

An exploratory study on the accuracy of the tracking system has been conducted. For each posture, 200 random frames from the testing set were extracted from the recordings of 2 of the subjects and processed offline by the tracking algorithm to detect the body parts of the cats and calculate the corresponding orientation vector. Table 4.1 shows a summary of the results indicating the percentage of frames for each posture in which the algorithm correctly identified the body parts and orientation vector. This initial validation shows a promising research line and future experiments will be conducted considering different classification algorithms and machine learning techniques in order to improve the accuracy of the system. 

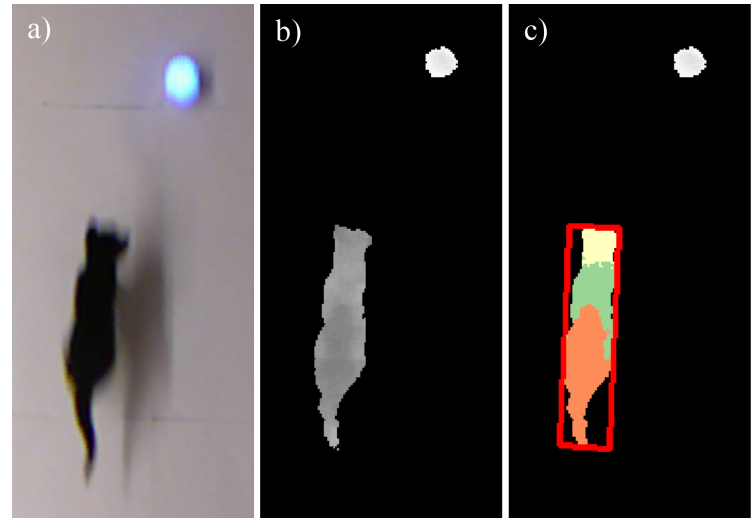

Figure 4.3: Cat running after a Sphero ${ }^{\circledR}$ robot.

Table 4.1: Accuracy of the tracking system identifying the cats' body postures and orientation vector.

\begin{tabular}{c|cccc}
\hline Posture & Sitting / semisitting & Walking / Standing & Jumping & Turning \\
\hline Accuracy & $86 \%$ & $74.5 \%$ & $82 \%$ & $84 \%$ \\
\hline
\end{tabular}

\subsubsection{Human Tracking}

ACI playful experiences should be conceived for both animal players only as well as for animals and humans playing together, enhancing interspecies communication and interactions. The development of tracking systems for humans using depthbased sensors placed on the ceiling would help to create new forms of natural interactions between humans and animals based on gestures and body postures rather than using actions on a specific device such as a screen or tablet. The first step in developing a human tracking system for this purpose would be extracting common human gestures, postures and behaviors during playful activities with animals. For instance, playing with our animals at home is a relaxed and informal activity, and humans usually adopt comfortable postures which allow proximity and confidence with their pets. It is likely that the human sits on the couch or on the ground, near to the pet, or even kneels on the floor, so both the human and the animal share the same play area and can interact directly with each other. In this situation, a top-down depth sensor will provide better contextual information of the human's relative location and interactions with the animal. A preliminary session was recorded to give some hints on the types of human postures and gestures to expect during these playful activities (see Figure 4.4). 

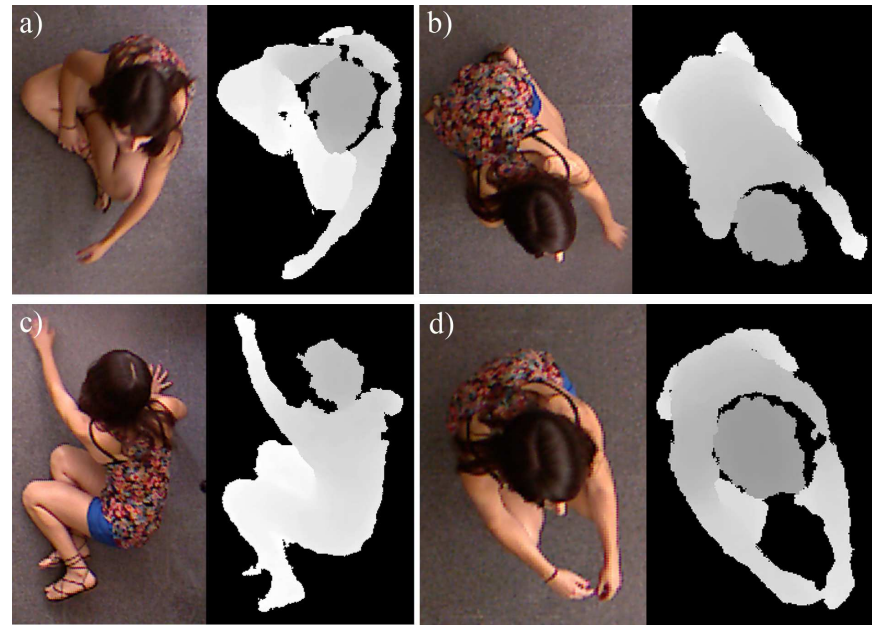

Figure 4.4: Human postures and gestures during play: a) sitting b) kneeling c) sideways d) crouching down.

In the same way that several sessions with cats were recorded to assist in the development of the cat tracking algorithm, further sessions should be conducted to record pets and humans playing together, creating a knowledge base to which we could apply computer vision algorithms to detect and learn human gestures during play. There is already an extensive research area in the field of computer vision which studies human tracking and gesture recognition. Approaches like the ones in $[20,130]$ would be a good starting point to recognize common body gestures adopted by humans playing with their pets. These gestures could later be used inside the game to perform actions, or to adapt the game to the interactions and postures of both humans and animals.

\subsubsection{Object Tracking}

With the current rise in the use of personal drones and robots, it is likely that games with animals will also evolve to incorporate such objects into the playful experience. These devices can be controlled by humans using tablets or smartphones, or they can be programmed to perform different movements or actions. In the latter case, the robots are not usually aware of their environment and preprogramming behaviors to be performed during the game could represent a threat to the animals' welfare.

These robots could also be tracked using the same approach: discarding the floor pixels from the depth stream and extracting the robots' contours. For instance, in Figure 4.3, a cat is chasing a Sphero ${ }^{\circledR}$ and this robot appears as a small blob in 
the image, which is easy to identify and track. Knowing the exact location of the robot and the cat inside the play area would allow us to define safety rules in the system to ensure the robot is not going to approach the animal in a dangerous way or perform risky actions. In addition, tracking the position of the robots together with the cats' location, orientation and body posture would allow to create novel and engaging playful experiences, as described later in this paper.

\subsubsection{Challenges}

At this development stage of the algorithm we are capable of tracking cats, humans and objects separately. The next step in the development process will be the integration of the three tracking mechanisms into a single system to allow collaborative and interspecies interactions with the intelligent objects in the game.

Currently, the tracking system only relies on single frame information in order to classify the pixel regions and detect the cat's body parts. However, temporal information of past frames would be very useful in the detection and classification of ambiguous contours and postures, as well as in improving the accuracy of the already detected postures. For instance, a probability orientation vector could be defined using the last n-vectors calculated. This vector could be used in cases such as in Figure $4.5 \mathrm{c}$ and $4.5 \mathrm{~d}$ in which the cat bent down and the tail could not be detected, causing the orientation vector to take a significantly different direction from the one in the previous frame (see Figure 4.5a and 4.5b).

Another challenging issue to be solved is related to the definition of the orientation vector: there are some cases in which the orientation vector does not always point to the area the cat is looking at, as can be seen in Figure 4.6. Relying on the center of the clusters to define the orientation vector leads to these situations. A solution to determine the real area the cat is looking at could be applying computer vision algorithms to the detected head contour in order to learn and detect possible head shapes. Another solution would be to calculate the major axis of the detected head cluster, which in most cases corresponds to the real orientation of the cat's head.

\subsection{Applications}

\subsubsection{Intelligent Playful Environments based on Gesture and Posture Recognition}

Tracking systems such as the one presented in this paper have an essential role in the development of future intelligent playful environments for animals. A previous study on cats' interest in different kinds of stimuli $[253,260]$ showed that in 


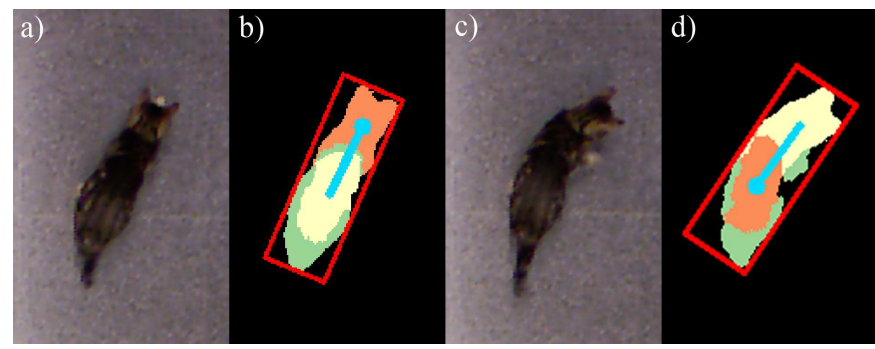

Figure 4.5: a) and b) Correct classification c) and d) erroneous classification due to ambiguous posture.
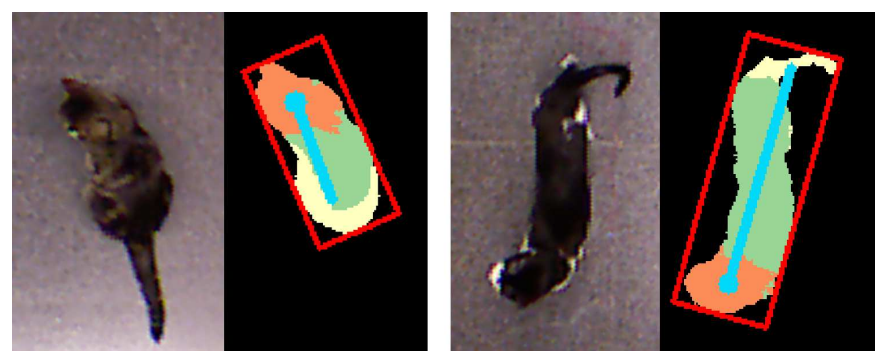

Figure 4.6: Orientation vectors differ from the heads' current orientation.

order to keep the cats' interest in the technological artifacts deployed in the study, some kind of "intelligence" is required. Random movements or actions of a digital element would cause the animal to eventually lose interest in the activity. Hence, being able to interpret an animal's body posture and interactions in a similar manner humans do, especially those suggesting either engagement or disengagement as well as distress signals during the game, is very important for providing proper actuations in the playful environment. Therefore, a tracking system capable of detecting a cat's location, posture and orientation would allow us to create engaging and realistic games using technological artifacts which adapt to the detected cat's postures and field of view.

Detecting the location of the cat inside the play area is essential to creating any kind of game which includes technological elements that appear and interact with the animal inside this area, such as robots or digital projections. This would mean, for example, the game could start whenever the cat approached a technologicallymediated toy inside the play area, and end when the cat runs away from the toy. The automatic detection of the cat's orientation would allow enriching and realistic playful activities to be created in which the technological artifacts could use the cat's area of interest, understood as its field of view, as the region on which to 
deploy the necessary stimuli to attract and maintain its attention. For example, a floor projection which passes in front of the cat's field of view and then moves away from him, encouraging the beginning of a chasing game. Posture detection is also a very promising feature because, together with the cat's field of view, it is an indicator of the cat's intentions towards the game. For instance, if the cat lies down and stops looking at the object involved in the playful activity, it is likely that he is not interested any more in the game. On the other hand, it is possible that the cat slowly approaches the object, e.g. a small robot, to jump over it when it is not moving, and the system could detect the cat's playful behavior by the position of his tail and the smoothness of his movements. In this situation, the system could make the robot move at the very last moment, surprising the cat and engaging him in the activity by imitating human-like playful interactions.

The automatic recognition of human gestures by a top-down depth-based tracking system would allow humans to participate in the game in a natural way for both the animal and the human, not being tied to any specific device. Human gestures could be used to control the movements or features of the digital elements in the play area, e.g. the human player points at an element with his hand and then points to another place, and the element moves in the direction indicated by the human. Some games could introduce specific gesture-based interactions, e.g. a competitive game between the animal and the human in which a clapping gesture makes the system move a toy/cord for 10 seconds to distract the cat so the human can take advantage.

\subsubsection{Learning Behavioral Habits}

Pet owners as well as animal caretakers in zoos or shelters easily identify the mental and physical state of the animals they are in charge of by analyzing their posture and movements. They are also capable of anticipating what the animal will do in some situations, as they have learned his behavioral habits and routines. For example, a human knows the time of day each of his pets prefers to play, the behaviors or movements that indicate that the animal is willing to start a playful activity and the meaning of the pets' interactions during the game. In a similar way, zookeepers know the routines of many of the animals they look after: the path that the animal will follow inside its habitat, the routine in which the animal prefers to drink, eat, sleep, play or walk, and even the animals' preferred spot for each interaction. This knowledge is acquired through daily observation and coexistence and it is difficult to transfer to another person who does not share the same context. In addition, it is also difficult to integrate this specific knowledge into a playful digital system, as each animal's preferences and routines would differ: generic knowledge would not cover the singularity of distinct individuals, while defining specific knowledge for each individual is not feasible. 
Complete animal tracking systems such as the one presented in this paper coupled with machine learning algorithms would allow us to learn behaviors, habits and even playful dynamics of individual animals. In this way, a personalized knowledge base could be obtained for each animal, similar to the knowledge their human companions have about them. Through the tracking system we could learn, for example, the habitual location and movements an animal performs in a specific context, the amount of time a day it spends doing physical activity such as walking or playing, and the intensity of this activity. This knowledge base could be used to detect changes in behavioral patterns, such as increasing/decreasing physical activity or resting time, therefore supporting early detection of illnesses or other problems. On the other hand, not all animals perform the same interactions in play: during a chasing game, a cat might prefer to wait patiently until an object approaches him to catch it, whereas another cat might be more eager and prefers to run after the object. Therefore, the tracking system could help to learn the specific play dynamics of an animal during a game. An intelligent playful environment could use this information in order to adapt the game to the animal's play preferences.

\subsection{Designing Future Tracking-based Gaming Experiences}

\subsubsection{Tracking Systems for Open Spaces}

The tracking system described in this paper is only suitable for indoor detection, due to the sensor restrictions in terms of light conditions, field of view and connectivity. Other methods would have to be considered if we wanted to carry out animal tracking outdoors without using a wearable device, such as dogs in their yards, zoo animals in their ecosystems or wild animals in their habitats.

Currently, there is a wide range of commercial or even home-made flying drones with built-in cameras or even powerful infrared sensors. This type of device could be used not only to observe the animals and collect images from the flights for post-processing $[122,336]$, but to track the animals autonomously and in real-time using the drones without a human controller. Computer vision algorithms could be applied to the extracted frames when the drone is flying over the animal's open ecosystem to detect its shape. Once detected, the drone could be linked to that specific animal and follow it throughout the area, locating its position using the drone's GPS or relative coordinates, and analyzing the extracted images to detect the animals' body posture.

Non-wearable tracking systems in open spaces using drones would make it possible to study the animals' natural interactions in less restricted ecosystems, learn 
their behavioral habits and also detect abnormal behaviors or possible illnesses. There are already studies within ACI that propose automated behavior recognition and animal tracking in the wild [223]. Autonomous outdoor tracking systems with drones could help these studies in the fulfillment of their goals. In addition, intelligent playful environments such as the ones described in this paper for indoor spaces could also be created outdoors using drone tracking mechanisms. For instance, a dog could be playing inside the living room with his electronic ball, which is controlled by the system and reacts to his interactions. During the game, the dog eventually runs towards the garden and expects the ball to follow him, as a human would do if they were playing together. The system would detect that the dog has gone outdoors and would transfer the playful activity as well as the mediating digital elements to the new play area. In this case, a drone would be activated and the first step of the system would be to send the drone to the garden in order to detect the dog's position. Once it is detected, the drone would keep track of the dog and communicate its movements, position and posture to the system, as the indoor depth-based tracking system would do. In this way, the playful experience would be seamless and more natural for the animal, not being restricted to only a specific area in the house.

\subsubsection{Mixed-reality Games and Embodied Interactions}

Developing digital playful experiences exclusively for animal players is as important as developing interspecies digital environments in which humans are also active participants in the game. Playful digital environments with pets and humans are not difficult to envision, as the interaction with them is natural and familiar to us and does not entail any risk to our safety. However, playful experiences involving animals in zoos imply that the human and the animal have to be in separate spaces for safety reasons. In addition, as we are not used to interacting with these animal species, our playful interactions might not make sense in the animals' mental perception of the game, and vice versa. How could we create a joint playful experience for users of different species with different perceptions of their interactions and who cannot share the same physical space?

Mixed-reality games could come to the rescue in order to create a common game with separate playful spaces in which the human interactions in its own space are transferred to the animal space in a meaningful way, and vice versa [44]. For example, humans could play using natural body interactions and movements in their play area, which could be recognized by a Microsoft Kinect ${ }^{\circledR}$ tracking system as described above. Human actions on their side of the game could be transferred to the animals' play area, for example to a digital device the animals like to use during their games without human participants. A very promising interaction modality on the human side of the game would be to use virtual or augmented re- 
ality devices, such as the Oculus Rift ${ }^{2}$ or the Microsoft HoloLens ${ }^{3}$, to immerse the human into the animals' ecosystem. One step further would be to introduce more immersive and embodied interactions for humans using omnidirectional treadmill peripherals, such as the Virtuix $\mathrm{Omni}^{4}$, together with these virtual reality devices. With these embodied interactions, humans could control elements within the animals' ecosystem and also experience feedback from the animals' interactions. For illustration purposes, let's assume that the favorite toy of orangutans in a zoo is a huge plastic ball, as several zoo animals have been observed playing enthusiastically with these elements [374] (see Figure 7). The ball could have an electronic inner rotor which allows it to be controlled remotely, like the Sphero ${ }^{\circledR}$. A human patient in a hospital could be using the Virtuix Omni for rehabilitation purposes, moving inside a digital recreation of the orangutans' ecosystem that he is watching through the Oculus Rift glasses (see Figure 8). Similar to what Metazoa Ludens [44] proposed with hamsters, the real movements of the human could be transferred to the huge ball within the orangutans' ecosystem, so the orangutans could really chase and interact with the ball as they would usually do. The movements of the orangutans inside their real ecosystem could then be tracked by depth-based sensors or cameras mounted on flying drones, depending on whether it is an indoor or an outdoor scenario. These movements would be transferred to the avatar representation of the orangutans within the digital world the human is observing through the Oculus Rift device. In this context, the goal of the human within the game could vary depending on the welfare needs of the animal playing: if the orangutan is physically active, the goal of the human could be not letting the orangutan catch him (which means that the orangutan could not catch the ball); if the orangutan participating in the game is detected to have lost his appetite, the goal could be to lead the animal towards the food area, i.e., to move the ball towards the food. Regardless of the scenario, animals' mental and physical wellbeing must be a priority. As in indoor domestic playful environments, the system would always ensure the participants' welfare by monitoring their interactions and body postures, introducing safety rules to impede risky behaviors, stopping the activity if the animals show distress and keeping the playing time within healthy boundaries.

\footnotetext{
${ }^{2}$ https://www.oculus.com/en-us/rift/

${ }^{3}$ https://www.microsoft.com/microsoft-hololens/en-us

${ }^{4}$ http://www.virtuix.com/
} 




Figure 4.7: Tiger playing with giant plastic ball (image courtesy of Boomer Ball).

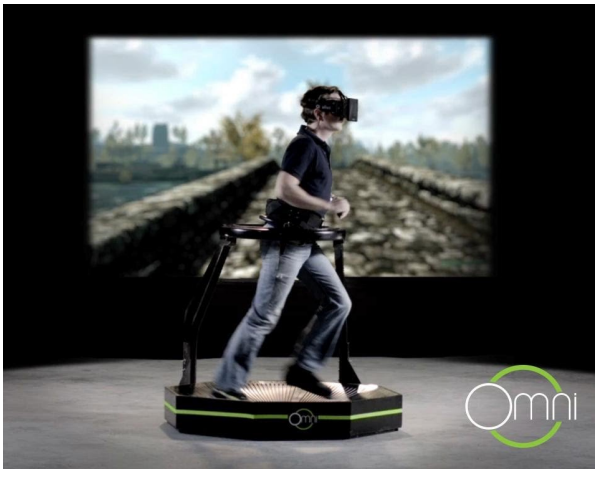

Figure 4.8: Human using the Virtuix Omni platform (image courtesy of Virtuix).

\subsection{Conclusions}

This paper describes the development of a tracking system for cats, humans and objects for indoor playful experiences, based on the analysis of depth information captured with a Microsoft Kinect ${ }^{\circledR}$ sensor placed top-down on the ceiling. The depth-based tracking system is capable of detecting a cat's location, body posture and orientation. It can also track humans and physical objects moving inside the tracking area.

Our immediate future work will be the integration of the three tracking modes into a single tracker. We are also working on improving the accuracy of the cat tracker by including temporal information from previous frames as well as studying its performance using different classification algorithms. After this, we will focus on the detection of human orientation, postures and gestures from a top-down point of view. This would allow us to create an intelligent playful environment in which the digital devices controlled by the system respond to the interactions of both humans and animals, attending to the contextual information of their bodies. We will also explore how this tracking system could be adapted to working with other pets, such as dogs, and zoo animals, such as orangutans.

We have also outlined future research directions in the area of playful environments for outdoor scenarios as well as for animals beyond pets. On one hand, we propose to explore outdoor tracking systems using drones and computer vision algorithms for different purposes, such as creating playful outdoor activities or learning animals' behaviors in open spaces. On the other hand, we believe that mixed-reality games for animals in restricted environments such as zoos could provide benefits for both animals and humans. On the human side, these games could play an educational role to raise awareness of the importance of wildlife 
preservation, as well as introducing more amusing ways of cognitive or physical rehabilitation. On the animals' side, these playful experiences would improve their wellbeing by introducing new forms of mental and physical stimulation adapted to each animal's context and preferences.

\section{Acknowledgments}

Work supported by the Spanish Ministry of Economy and Competitiveness and funded by the EDRF-FEDER (TIN2014-60077-R). The work of Patricia Pons has been supported by a national grant from the Spanish MECD (FPU13/03831). Alejandro Catalá also received support from a VALi+d fellowship from the GVA (APOSTD/2013/013). Special thanks to our cat participants, their owners, and our feline caretakers and therapists. 


\title{
Chapter 5
}

\section{Towards Future Interactive Intelligent Systems for Animals: Study and Recognition of Embodied Interactions}

\author{
Patricia Pons ${ }^{1}$, Javier Jaen ${ }^{1}$, Alejandro Catala ${ }^{2}$ \\ ${ }^{1}$ ISSI Group, Department of Computer Systems and Computation (DSIC), \\ Universitat Politècnica de València, Valencia, Spain

\footnotetext{
${ }^{2}$ Human Media Interaction, University of Twente, Enschede, The Netherlands
}

Published in Proceedings of the 22nd International Conference on Intelligent User Interfaces, pp. 389-400, ACM (2017). doi:10.1145/3025171.3025175

\begin{abstract}
User-centered design applied to non-human animals is showing to be a promising research line known as Animal Computer Interaction (ACI), aimed at improving animals' wellbeing using technology. Within this research line, intelligent systems for animal entertainment could have remarkable benefits for their mental and physical wellbeing, while providing new ways of communication and amusement
\end{abstract}


between humans and animals. In order to create user-centered interactive intelligent systems for animals, we first need to understand how they spontaneously interact with technology, and develop suitable mechanisms to adapt to the animals' observed interactions and preferences. Therefore, this paper describes a pioneer study on cats' preferences and behaviors with different technological devices. It also presents the design and evaluation of a promising depth-based tracking system for the detection of cats' body parts and postures. The contributions of this work lay foundations towards providing a framework for the development of future intelligent systems for animal entertainment.

\subsection{Introduction}

Interactive systems for entertainment and games have brought countless benefits to human wellbeing in a wide variety of scenarios [85, 210, 237, 301], and the incorporation of intelligent features into these systems has been observed to strengthen their advantages and improve the user experience [63, 309, 318]. Interactive and adaptive playful experiences could have benefits not only for human beings, but also for non-human animals - to which we will refer from now on in this manuscript just as animals for the sake of clarity and simplification. Animals coexist with our technologically filled environments, and both domestic and non-domestic animals show interest in our human-centric entertainment devices, such as televisions, tablets and smartphones [113, 297, 352]. However, due to both their different physical features and mental processes [197], current technologies or interaction mechanisms might not be suitable for them if the design does not take into account their capabilities. For example, interaction mechanisms that may seem natural to us, such as direct touch finger manipulation, tend not to be applicable to some animal species, e.g. birds or dogs. Following a user-centered perspective, the currently growing research field of Animal Computer Interaction (ACI) $[173,174,222]$ considers animals as target users of digital systems and aims to design and develop suitable animal-centered technology. One of ACI's main goals is to improve animals' wellbeing and capacities by adapting technology to them, in the same way that HCI has improved humans' wellbeing by adapting technology to address human needs: monitoring systems could help in the detection of changes in behavioral patterns and early detection of illnesses [214], remote communication systems based on wearables and gesture detection could ease the task of Search and Rescue Dogs [30], and specifically designed devices could help medical assistance dogs to alert emergency services when their owner is at risk [280].

A very promising area of research within ACI focuses on improving animals' wellbeing by means of playful technology [17, 78, 258, 351, 363]. Play is one of the most natural behaviors among all species [131], and it is especially important for non-human animals. It teaches them behaviors they need to acquire in their 
adult life. It also helps to keep their minds and bodies active, reduces stress and could be an indicator of well-being [258, 363]. At the present time there is a lack of diverse and complete games for domestic animals, as these have not evolved very much from the throw-and-catch interactions for dogs, or chasing interactions for cats and their toys. Not only pets, but also animals in shelters or zoos need enrichment activities to ensure their wellbeing [240, 342]. Although there are a few proposals, such as electronic ball throwers for dogs or initiatives such as Apps for Apes [10] which allows orangutans to play games on tablets, they present some drawbacks. Firstly, the dynamics and interactive elements of the game do not change based on the animal's context and, secondly, when the animals are left alone they are unable to play with the technological elements. Given that animals need both personalized physical and mental stimulation [198, 347], single-toy, nonadaptive and always human-mediated playful technology might not offer them all the enrichment they need.

In order to cater these needs, intelligent systems for animal entertainment would be a very promising way of providing new forms of mental and physical stimulation $[254,258]$ as well as allowing animals to play by themselves, without requiring a human to be present providing the technology. These interactive playful environments could be comprised of multiple devices in order to provide multimodal interactions and suit the needs of different animal species or even individuals within the same species. As animals mostly rely on body language to express themselves, the system could analyze the behavior of the animal and then manage those devices to automatically adapt their responses to the animals' embodied interactions. This is usually what happens when for example a human is playing with her pet, i.e., she observes the dog's wagging tail and crouched position and interprets it is time to throw the ball. In this way, engaging and innovative playful scenarios could be created, which foster both mental and physical activity and improve the animals' wellbeing in several ways. For example, a digital game could consider the position of an animal and its body posture in order to adapt the content of the game to the behavior of the animal, e.g. if the animal is overexcited or tired, the game speed could be slowed down, whereas if he gets bored, the system could introduce new elements into the game to keep his attention. In addition, humans could now intervene in these playful activities as participants, even remotely, and not just as providers of the activity.

With the foregoing in mind, one of the first steps to develop suitable intelligent environments for animal entertainment would be to understand how our endusers, the animals, spontaneously interact with different technological stimuli and behave with computer-mediated systems. This acquired knowledge would allow the appropriate metaphors and artifacts to be selected when developing engaging animal-centered interactions. The study of animals' playful interactions with technology would also help us to design suitable mechanisms to unobtrusively extract 
contextual information so that intelligent systems could adapt their responses to the animals' interactions and preferences.

This work is a first step in this direction and proposes, to the best of our knowledge, the first observational study which analyzes the interactions and preferences of cats towards different technological artifacts. In this respect, we discuss how the proposed technologies could be used effectively in future intelligent environments for cats depending on the observed interactions and preferences. In light of the observations, we also present the development and evaluation of a promising tracking system for the detection of the observed cats' interactions within intelligent playful environments. This is the first non-wearable tracking system capable of detecting cats' postures, body parts, location and orientation using depth-based information. Overall, the results presented in this paper would provide very valuable foundations to inform the development of suitable intelligent environments for animal enrichment.

\subsection{Related Works}

\subsubsection{Technologically Mediated Playful Systems for Animals}

Even before the field of ACI emerged, there have been several works studying playful technological interventions for animals.

An exploratory study with dogs has been conducted in order to study the nature of dog-tablet interactions with several pre-existing tablet-based games for animals $[15,16,17]$. Results report that some dogs showed distress signs, reinforcing the idea that adaptive user-centered designed games which really support the animals' preferences are required. In this regard, there are several studies focused on specifically designed tablet-based games for cats. Cat Cat Revolution [224] is a digital game for iPad which shows an animated mouse moving across the screen the cat has to catch. The digital mouse can move randomly across the display, or can also be controlled by a human. Felino [351, 352] is an interspecies game designed using ACI principles. The game allows a human and a cat to play together catching elements which appear on a shared tablet screen. The human can adapt the game to the cat's reactions and preferences. However, these works focus on just one single game and interaction modality, and the human is responsible for the adaptation of the game's responses and features.

There have been several studies with non-domestic animal species which provide several interaction modalities and devices in order to evaluate the animals' spontaneous interactions and preferences with them. On one hand, specific devices for elephants, such as different types of buttons and activators, are being developed and evaluated $[75,79]$. The goal is to select which mechanisms are preferred by 
the elephants to control playful features that will later on be introduced into their environment. Playful interactions with orangutans have also been studied. One of the enrichment solutions used in zoos has been the use of tablet-based games that researchers or caretakers have to hold towards the animals' enclosure [10]. The use of touchscreens inside the enclosure has also been evaluated [240, 361]. Another solution has been the design of specific tangible devices which make use of already known metaphors by the orangutans, such as poking with sticks [142] or moving objects [247], to produce sounds. However, within these works the human needs to provide and/or take care of the technology and therefore animals cannot play when they are left alone. And again, most of the provided devices do not account for autonomous personalization and adaptation features.

Wirman [364] states that the study of animals' playful interactions with familiar objects could inform the design of playful and intuitive mappings to technological objects. In our proposed observational study, we will analyze cats' intuitive and spontaneous behaviors with different technological artifacts that could be part of a playful experience. This will inform the design of an intelligent playful system that would adapt the reactions of its artifacts to the animals' intuitive interactions.

To enable the system to autonomously adapt to the animals' interactions, we also need to define ways of extracting information from the animals' observed body language which gives us context on their intended behaviors. The next section will therefore analyze current technology for animals' body posture and behavior identification.

\subsubsection{Animals' Behavior and Posture Recognition}

Most of the works on animals' behavior recognition rely on wearable sensors to gather information about the animals' postures and movements, which are then processed using machine learning techniques. Canine Amusement and Training [359] is a training system for dogs consisting of IR emitters attached to the dog's harness, and a Wiimote's IR camera placed on the ceiling. The system detects the location and posture of the animal by tracking the IR emissions of the harness using the Wiimote. Another work has studied the recognition of motion-based dog gestures - sit, spin, roll, jump, etc. - using a three-axis accelerometer attached to the front of a service dog harness [328]. More recently, they have studied the use of a dog collar with an accelerometer and gyroscope for the recognition of head gestures on dogs [329]. In [158], dogs wear a tri-axial accelerometer on the collar and, after being trained with a $\mathrm{kNN}$ classifier, the system is able to differentiate between 14 activities and 2 postures. The work in [30] uses a similar approach by placing more sensors along the dog's harness and then applying machine learning techniques to recognize up to five static postures and three dynamic behaviors. They have also compared the performance of the classification algorithm using supervised against unsupervised classification methods [360]. 
However, playful scenarios require more freedom of movement and some animals might not feel comfortable wearing new devices that could somehow limit their spontaneity. Although there are a few examples of non-wearable systems for animals' behavior recognition $[148,164,323]$, these systems are only capable of detecting the location of the animal but no postures nor body parts are detected. In this paper, and after observing how cats intuitively interact with different technological elements, we will describe the design, implementation and evaluation of a non-wearable system capable of recognizing cats' postures, body parts, location and orientation during a playful activity.

\subsection{Playful Interactions with Technology}

There is growing interest in studying animals' playful interactions with technology within the ACI community. In order to design user-centered systems which respond to the animals' interactions in an appropriate manner, first we need to study how animals spontaneously interact with technology. Moreover, there are many different technological devices and interaction modalities that could be part of an interactive environment for animals. Depending on the species, the interest of the animal in a specific stimulus/device might change. In addition, the animals' personalities could also have an impact in these preferences [24, 239, 314]. For these reasons, intelligent systems would be of extreme value in order to gather the context of the interactive experience and adapt to it, i.e., learn what the animal preferences are and adapt to its interactions in a suitable way.

In order to evaluate which factors could affect these interactions, which devices would initially be more suitable, which are the observed interactions and how the system could adapt to them, an observational study has been conducted in order to evaluate cats' interest in different kinds of technologically-based stimuli and interaction modalities [253]. This section will describe in detail the conducted behavioral study, the activities and interactions that cats performed with different types of technological devices, an in depth discussion of identified cats' spontaneous reactions and interactions, and potential factors affecting the interaction. The cat species has been selected due to the lack of technological suitable solutions for them, however the methodology could be applied to study other animal species.

\subsubsection{Observational Study}

\section{Subjects}

Seven different cats participated in this study: 4 kittens of ages ranging from 2 to 4 months and 3 adults of ages from 2 to 5 years old. The cats were brought to the study by private pet owners and some also came from a local shelter. The humans 
accompanying them were thus either their owners or their caretakers. All of the cats were used to socializing with human-beings as well as with other animals. All of them were once stray cats: four of them were adopted by their current owners during the first six months of their lives, two are currently being cared for in private houses and one was adopted after stays in seven different caretakers' houses.

\section{Technologically-based Stimuli and Interaction Modalities}

In order to assess cats' interest and spontaneous interactions with different technological elements, a list of potential appealing stimuli of different types was defined. This decision process was discussed with experts in feline behavior and caretakers in order to assess which stimuli (and technological devices) would potentially be the most attractive ones for the animals. The first decision was to select which senses would be stimulated, i.e., sight, hearing, smell, taste or touch. For cats, sight and touch were the stimuli that both researchers and experts decided to incorporate into the first iteration of the study. Then, several technological solutions capable of providing each of the proposed stimuli were outlined.

Sight and touch are interrelated factors when it comes to selecting a technological device that addresses them. For example, a visual stimulus can be implemented by a digital or a tangible object that is able to move around. Regardless of its nature, an object can describe a trajectory in many different ways when it moves. Thus, features such as the intensity, duration and length of an object's movements could make a difference in the cats' perception and interest: when cats chase their prey or play using chasing behaviors they sometimes require fast and energetic movements of the prey/toy to jump towards it, while sometimes slight movements of a toy/hand cause them to approach the object and touch it curiously. An intelligent system should be able to replicate these interactions if they are detected as engaging for the cat. Two movement dimensions were therefore considered: inplace movements, i.e. slight, sudden movements of an object, and long distance movements, i.e. long and fast movements which encourage chasing.

\section{Equipment}

The room in which the study took place was $11.80 \mathrm{~m}$ long and $7.20 \mathrm{~m}$ wide, with a play area of 180 centimeters long and 200 centimeters wide of white cardboard placed on the floor. However, the cats could explore the room freely and walk away from the activity at any time in order to ensure their comfort and wellbeing. A Microsoft Kinect ${ }^{\circledR}$ sensor was used to record video streams of depth and color information from the cat's movements during the sessions. This information was later analyzed to identify play dynamics as well as common cat body postures during play, as will be explained later on in this paper. The sensor was placed 
on the ceiling, facing down, at a height of $250 \mathrm{~cm}$ in the center of the projection area, so depth and color streams from the defined play area could be recorded (see Figure 5.1).



Figure 5.1: Set-up of the observational study: play area and location of the projector and depth sensor.

For the digital stimuli, a projector was used to display images on the floor of the play area (see Figure 5.1). The projector was fixed to the ceiling at a height of $260 \mathrm{~cm}$. The supporting structure allowed the projector to tilt at an angle of 60 degrees. The projector applied keystone correction to the displayed images to rectify the tilt angle. The projected images were $180 \mathrm{~cm} \mathrm{x} 200 \mathrm{~cm}$, and covered the entire play area. The projected elements were in the form of cartoonish mice. The size and color of the digital mice as well as the background color of the image were adapted and pre-tested with two cats during preliminary sessions to ensure that the cats were able to see them. After testing with different prototypes, the final version consisted of yellow mice of approximately $5 \mathrm{~cm} \mathrm{x} 5 \mathrm{~cm}$ on a black background.

Two small robots were used for the tangible stimuli: a Sphero ${ }^{\circledR}$ (see Figure 5.2, Left) and a Parrot ${ }^{\circledR}$ Jumping Sumo with a tangible toy attached (see Figure 5.2, Right). The first robot, Sphero, is an electronic ball which can be controlled via Bluetooth through an Android or iPad application. It also glows and its colors and brightness can be changed. Sphero's size, movement speed and luminosity make it a viable option to capture a cat's attention. The second robot, the Parrot Jumping 
Sumo, is not much bigger than Sphero but has wheels and does not resemble an object the cats would be familiar with. It was selected because of the smoothness and agility of its movements as well as its physical features: it has a retractable piece on its back which allows a tangible cat toy to be attached to it, which could make the device look more friendly or familiar. It can also be controlled through an Android or iPad application via Wi-Fi. Both robots incorporated predefined movements which varied in intensity and length: some of these were gentle and strictly in-place, while others were energetic and entailed movement of the robot a few centimeters. These predefined movements were included to study the type of movements with tangible objects that could be potentially interesting for cats. Both robots can be programmed through an SDK, which would allow them to automatically move around the play area in future development stages of playful scenarios for animals.

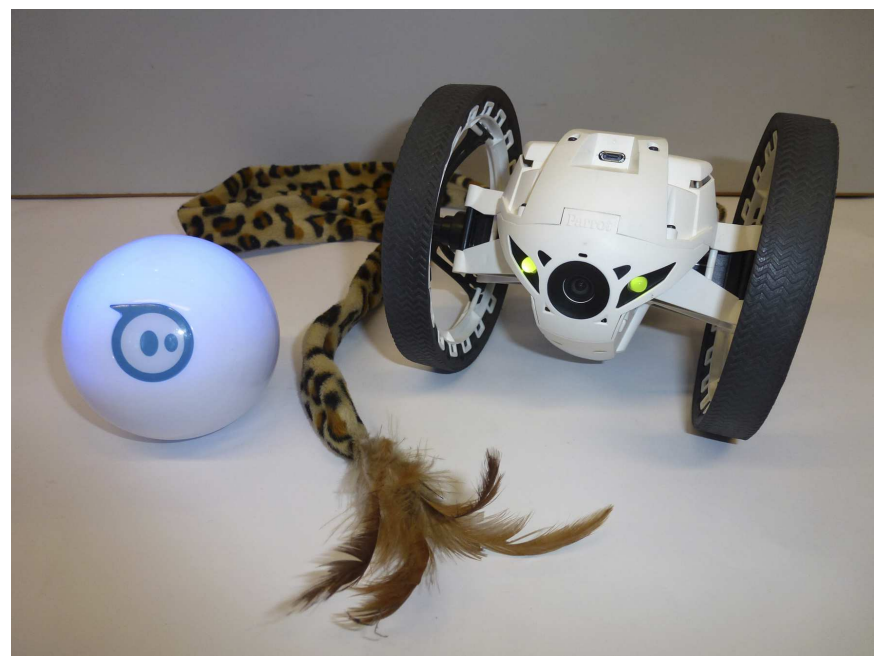

Figure 5.2: Sphero (left) and Jumping Sumo (right), the two robots used in the study.

\section{Procedure}

The study took place in a room in which the cat participants had not been previously. It was expected that the new environment would cause some distractions during the experiment. However, it was not feasible to conduct this study at the shelter nor at the cat owners' house due to the set-up requirements. The cats were given some time at the beginning of the session in which no technology was activated and they could explore, recognize and mark the room. During the study the cats could move and explore freely, and they could leave the interaction area whenever they wanted to. If a cat showed boredom or frustration the activity 
was stopped and the caretaker or owner provided the cat with either a reward or played with him and its favorite traditional toy. Ethical guidelines for ACI studies were considered $[175,325]$.

During the session, at least one feline caretaker or therapist was present, who helped to interpret the cats' reactions, behaviors and preferences for each stimulus. All the activities were presented to each of the subjects. The duration of each activity varied according to the interest of the cat. During each activity, the corresponding stimulus was triggered repeatedly for a certain time using a Wizard of $\mathrm{Oz}$ approach: a researcher was controlling the technology in order to simulate the reactions of an intelligent system when the animal interacts, following recommendations from the feline expert. Observational feedback was gathered from each activity and subject. The total duration of each session was around one hour. A camera with both depth and color sensors pointing down from the ceiling was used to record the cat's movements and body gestures during play.

\section{Activities}

- Activity 1: In-place movements with a single digital mouse. A single digital mouse was projected onto the ground performing in-place movements consisting on slight, sudden movements around its original position.

- Activity 2: In-place movements with multiple digital mice. Several mice were projected as it was suggested that the number of digital elements could affect the interest of the animals: some animals might be interested in the game with only one digital mouse, while others might require more elements to attract their attention.

- Activity 3: Long distance movements with a single digital mouse. In this activity, the researcher controlled the digital mouse and adapted the long distance movements to the cat's reactions in order to simulate a real chase: moving the mouse towards or away from the cat and performing long movements far away from the cat.

- Activity 4: Long distance movements with multiple digital mice. In this activity, the digital mice were programmed to perform predefined movements in the form of sinusoidal paths with random length and starting points. The number of mice did not change during the activity. Each mouse started a new movement from the final point of the last movement it performed so that the mice did not disappear of the screen during the activity (see Figure $5.3)$.

- Activity 5: In-place movements with Sphero. Sphero robot controlled by the researcher, performing different predefined in-place movements (rotation, jumping, etc.). 
- Activity 6: In-place movements with Jumping Sumo. Jumping Sumo robot controlled by the researcher, performing different predefined in-place movements (rotation, turning around, spinning, etc.).

- Activity 7: Long distance movements with Sphero. Sphero robot controlled by the researcher, performing long distance movements around the play area, varying in intensity, length and path according to the cat's reactions and following the instructions of the caretakers.

- Activity 8: Long distance movements with Jumping Sumo. Same mechanism as in Activity 7.

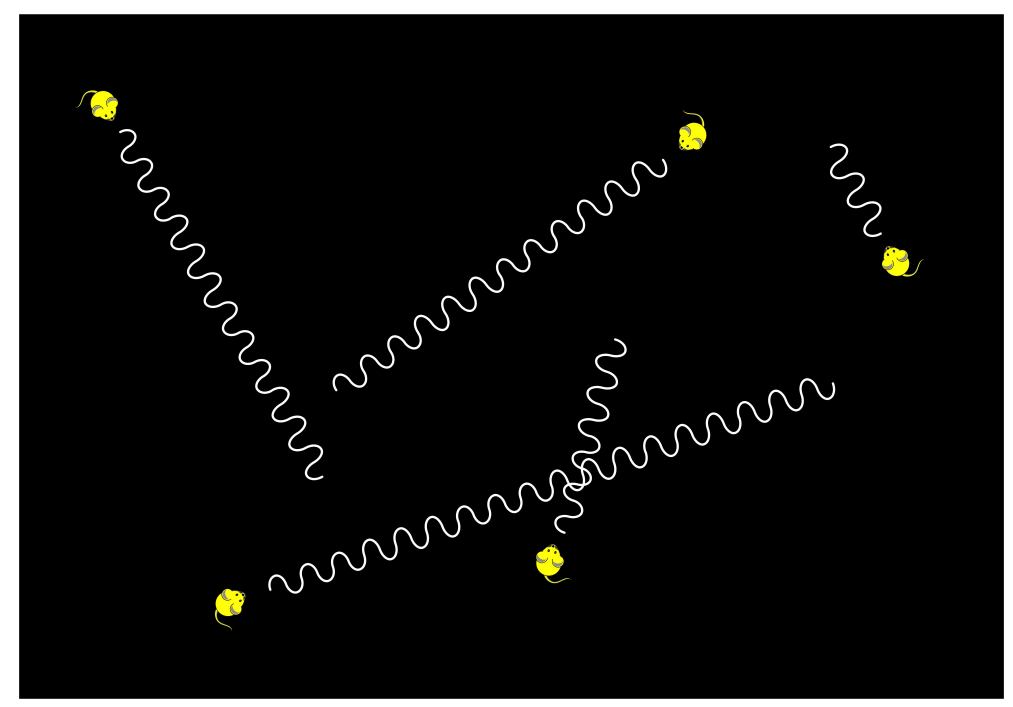

Figure 5.3: Different paths of the mice in Activity 4.

\subsubsection{Results and Discussion}

Although cats' reactions and interactions were very different between individuals, several similarities could be found especially when the animals engaged into playful interactions with a specific technology. The results outlined in this section could be used as initial rules an intelligent system could follow to start an appropriate interaction depending on the characteristics of the animal user, such as age, size and initial attempts to approach the technology.

In light of the observations, the cats' age and size seemed to have influenced their preferences. The adult cats did not show any refusing behavior towards any of the tangible stimuli. However, three of the four kittens were insecure during 
their first attempts with the tangible stimuli, and finally the three of them played to a greater or lesser extent with the smaller of the two robots, Sphero (see Figure 5.4 ), while they practically refused to play with the bigger one. The size of these robots could have prevented the kittens from considering them a prey or even a toy, and they only ended up feeling comfortable with the smaller robot. It could be so because this robot, Sphero, is in the shape of an electronic ball, and kittens may be used to playing with a real one. Only one of the four kittens played almost immediately and with the same interest with the two robots, and this was presumably caused by the inherent curious personality of this specific cat, as it was the smallest of the four kittens and its size was likely to prevent him from playing with this kind of elements. In general, smaller tangible elements should be used for small cats, as these devices/artifacts must resemble a real prey the cat would want to catch.

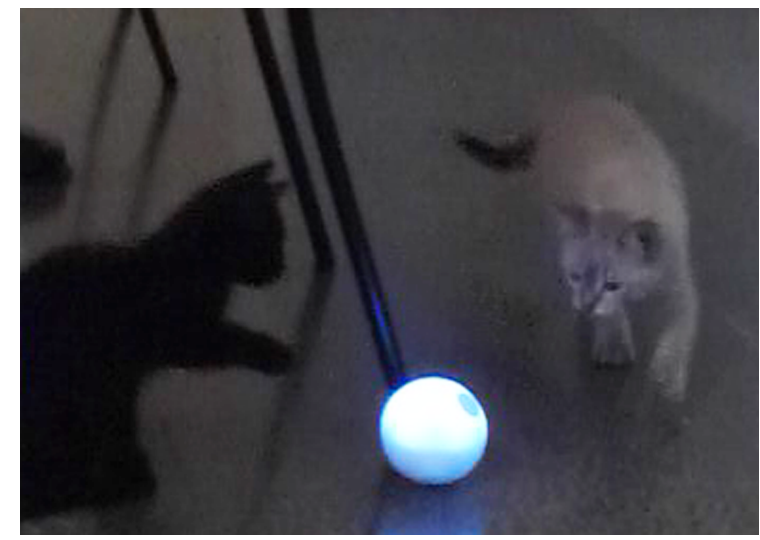

Figure 5.4: Two kittens playing with a Sphero ${ }^{\circledR}$.

During the study, the adult cats showed more interest in tangible visual stimuli than in digital ones. Age might not be a determinant factor in this case, as different preferences between tangible and visual stimuli were observed in the kittens. However, it was observed that the novelty factor of the digital stimuli may have affected the interaction of some of the animals. Adult cats are used to playing with laser pointers, and the visual digital stimuli used in this study might remind them of traditional laser pointer games. Consequently, the novelty of the tangible robots might have affected the degree of interest of these cats in favor of the tangible stimuli. This shows that incorporating several technological elements might help in keeping the animals' interested. If the system detects the cat is not interested in a specific device, it could change to a completely different artifact (technology or interaction modality). Moreover, the system's ability of changing the behavior of the artifacts over time might play a very important role in maintaining the novelty factor. In addition, as experts do not recommend laser pointer 
games, the visual digital stimuli included in this observational study should in the future be accompanied by other kind of stimuli and/or rewards to complement the activity and avoid the cat's frustration for not being able to catch the light. Regarding the tangible stimuli, it was observed that incorporating familiar elements, such as a common cat toy (see Figure 5.2, Right), to a robot's physical structure increased the interest of the cats in the stimulus, and the interactions with this robot were focused on catching the toy rather than touching the physical structure of the robot.

The familiarization factor should also be considered when introducing new elements into an animal's environment. Some cats might need more time to familiarize themselves with new stimuli and elements in order to show the same interest in it, unlike other cats who quickly start the interaction. It appears from the observations that this was not an age-related factor. Two of the kittens immediately or almost immediately showed interest in some of the tangible and digital interaction modalities, while the other two did not show any interest in the digital stimuli. It also took them some time to get used to one of the tangible stimuli, which they eventually played with. This should be considered by the system when starting the interaction. For example, if the body language of the animal indicates uncertainty or shyness, e.g., the animal approaches the device slowly or does not approach, the reactions of the technological artifacts should be smoothed to accommodate to the animals' familiarization pace.

The ability of the cats to keep eye contact with the source of the stimuli was observed to be affecting their interest in the activities with digital elements. When the cats were playing by chasing the digital mouse controlled by the researcher (Activity 3), they eventually jumped or tried to chase the mouse. This caused them to lose eye contact with their digital prey, which had been moved to another place, and the cats were seen to be looking around for it. In this case, the researcher could control the movements of the digital mouse, and placed it in a visible location for the cat to maintain its attention. However, if the system was autonomous, the lack of information on the cat's position and orientation, i.e. the cat's current field of view, would force the mouse to move randomly across the play area. This simple behavior would make the cat lose interest in the game or get annoyed. On one hand, the inability to quickly locate the mouse might frustrate them and, on the other, if the chase does not evolve intelligently and imitates a real chase, the animal would get used to the activity very soon and lose interest. The same would eventually happen with tangible stimuli if the robots were controlled randomly by the system. Effective mechanisms which consider the animals' location and visual range should be developed with the aim of informing the evolution of the stimuli, keeping the animals interest on the game and guaranteeing their welfare.

An interesting behavior was observed during the activities with visual stimuli. As expected, the cats behaved with these stimuli as they would do in a real chase. It is known that cats do not just run after their prey. They are very patient 
hunters, and usually sit or lie on the ground, waiting for the right moment to catch the prey. For instance, the cat sometimes lay on the ground, ready to start running, but waited until the mouse or robot moved towards him and then jumped at it. Other times, the robot or mouse moved away from the cat fast, causing the animal to run after it. During the activities with the single mouse or the tangible robots, a researcher was controlling the movements of the objects, and a professional caretaker was giving instructions on how to perform these movements in order to gain the cats' attention. This created realistic and amusing chasing scenarios for the cat, which might not have been the same if the visual stimuli had been performing random movements instead of adapting to the cat's reactions. In addition, sometimes the cat lost sight of the digital mouse, which moved very fast or ended up behind the cat. In these situations, the researcher noticed the cat's confusion and moved the mouse into its field of view. As before, this would have been impossible if the mouse movements had been randomized. Therefore, if future playful environments for animals are aiming to be autonomous without requiring human presence for the animal to play, they should find an intelligent way to manage these scenarios. The following section will outline a promising solution for creating intelligent, autonomous and engaging playful environments which adapt themselves to the cats' interactions.

\subsection{Behavior Recognition Using Depth-based Tracking}

As previously discussed in the observational study, animals adopt characteristic postures and behaviors during playful activities which will be useful to identify and detect. When humans interact with an animal, either using a traditional toy or a technological device as mediator of the interaction, the body language of the animal and the aforementioned observed behaviors give a hint of what to do next to foster the animal's playful interaction or to understand that the animal is not interested in the activity anymore. Intelligent environments must be able to extract this contextual information and use it to evolve the game and create adaptive playful scenarios. Some of these postures observed during the sessions conducted with cats are described in Table 5.1.

These behaviors and postures of the animals during a playful activity are a very valuable source of information for the evolution of the game, e.g., if the cat is observed in a hunting position, the technological stimuli could be moved towards the animal in order to trigger an engaging chasing behavior. Being able to automatically recognize those behaviors and postures would provide suitable contextual information required for the development of intelligent interactive games which could run without requiring human supervision, autonomously adapting to the animals' interactions. Moreover, the automatic detection of the cat's orientation would allow enriching playful activities to be created in which the technological 
Table 5.1: Different cats' postures observed during playful activities.

\begin{tabular}{c|c}
\hline Postures & Description \\
\hline Standing & The cat is standing on its four legs, head slightly higher than the body \\
Walking & The cat is standing, head slightly bent forward and legs moving forward \\
Sitting & The cat is sitting on its rear legs, without bending its front legs \\
Themi-sitting & The cat is sitting on its rear legs but bending towards the front \\
Turning & The head of the cat and its front part of the body are curving towards one side \\
Jumping & The cat jumps on its back legs, head and front paws up, all body extended vertically \\
Hunting & The cat bends towards the ground in an alert position \\
Lying down & The cat lies on the ground on one side or on their paws \\
\hline
\end{tabular}

artifacts could use the cat's area of interest as the region on which to deploy the necessary stimuli to attract and maintain its attention. Determining the cat's field of vision at any given time, combined with the cat's body posture and location will allow us to create engaging and realistic activities. It will also solve the problems described in the last section regarding the evolution and adaptation of the activity in order to maintain the animal's interest in the stimuli. As the system is envisioned for playful interactive scenarios, non-wearable tracking systems would offer more freedom of movement to the animals without affecting their spontaneous interactions.

This section will therefore describe the design and development of a non-wearable tracking mechanism capable of recognizing basic cats' postures during playful activities, as well as their orientation and location within the tracked area. A prototype of the system was initially validated [257] to demonstrate its feasibility. Therefore, this section will describe the design, experimental procedure and detailed performance evaluation for the development of a tracking system for cats capable of differentiating between different postures and body parts using both supervised or knowledge-based classification mechanisms.

\subsubsection{System Description}

The tracking system has been developed using C\#, Microsoft Kinect ${ }^{\circledR}$ SDK 1.8, and EmguCV, an OpenCV framework for .NET systems which has been used for image processing. During the sessions, a Microsoft Kinect ${ }^{\circledR}$ v1.0 sensor was used as described in Figure 5.1 to record the cats' movements and behaviors during the activities. The camera and depth sensor provided an aerial view of the play area (see Figure 5.5). The Microsoft Kinect ${ }^{\circledR}$ v1.0 sensor provides both color (see Figure 5.5a) and depth streams (see Figure 5.5b). The tracking system will only use depth information as input, so the color streams are discarded. Each depth frame provides, for each pixel, the distance in millimeters from the camera plane to the nearest object in that particular pixel (see Figure 5.5b). Relying on depth information allows not only to differentiate between different body parts 
of an animal within the image, as could be done by computer vision methods, but also to exploit information about the location and depth of those body parts. In this way, an implicit 3D representation of the animals' body can be elicited. This provides a lot more information to differentiate between postures in which analysis of $2 \mathrm{D}$ images would not be conclusive. Moreover, these implicit 3D models of postures could be used to construct behavior models, ethograms, or activity recognition systems if temporal information and machine learning methods are also used. In the obtained depth images, the contours of the cats can be clearly observed as their depth values are greater than the floor's depth, which is constant. Instead, the depth pixels of a cat vary along its body, allowing a human eye to differentiate between the different parts of the cat's body just by looking at the depth frame. In the same way, different cat postures can be observed to generate different cat contours in the processed depth frames. For example, the cat's depth stream contour when sitting showed a smaller, square-shaped bounding rectangle (see Figure 5.5d, cat on the bottom of the image), while the depth stream when standing or walking showed a larger and rectangular-shaped bounding rectangle (see Figure 5.5d, cat on the top of the image).

The first processing step of the algorithm consists of extracting the cat's pixels from the depth frame (see Figure 5.5b). In the current implementation of the algorithm, which has been tested with prerecorded data, background segmentation has been done by simply discarding from the image those pixels corresponding to the ground, as the sensor was placed in a fixed position during the sessions and therefore the distance to the floor was known (see Figure 5.5c). However, this approach can unintentionally remove the cats' tail (as observed in Figure $5.5 \mathrm{c}$, cat on the lower-right corner of the image), which in some cases could be a source of valuable information when creating richer posture descriptors. Thus, more elaborated approaches for background segmentation should be incorporated

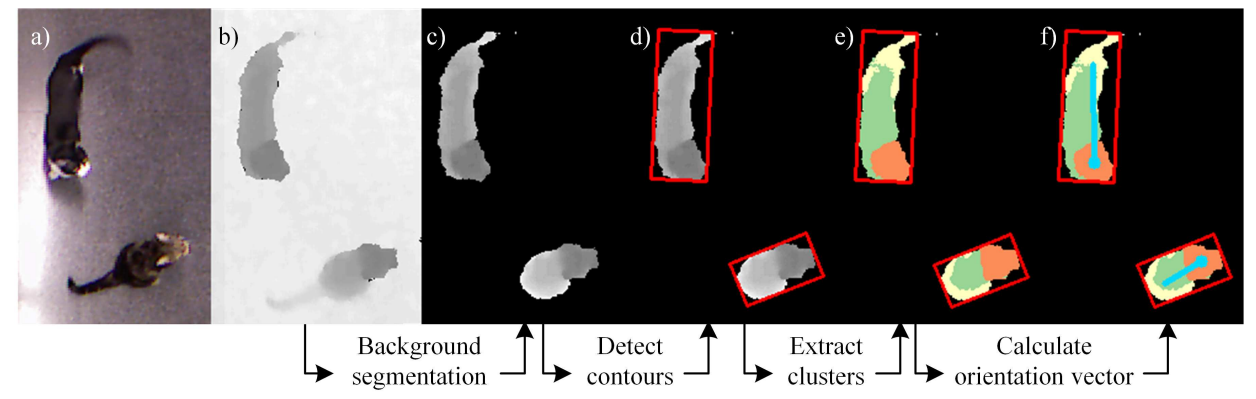

Figure 5.5: Process of extracting the cat's orientation and body parts: (a) color frame (b) depth frame, (c) background segmentation, (d) cat contours, (e) clusters for head, body and tail, (f) orientation vector. 
in order to overcome this current limitation and to allow for more flexible set-up conditions. With the floor removed from the image, computer vision algorithms are applied to the depth image in order to extract the cats' contours, which now appear as grey-scale blobs on the image (see Figure 5.5d). In this step, a cat's location within the tracked area can be determined by using the centroid of the extracted contours as a 2D coordinate. Each detected contour is then processed by a k-means clustering algorithm, which groups the pixels by their depth value and relative position (see Figure 5.5e): pixels of similar depth which are located together in the image would be grouped together within the same cluster. The number of clusters was set to three in order to divide the cat's contour into the three most noticeable parts of the cat's body, i.e. head, body and tail. The following step of the tracking system would be the recognition of the cats' postures and body parts. For this purpose, the obtained clusters of each cat have to be classified into either head, body or tail. Once the head is detected, its position in relation to the body/tail clusters allows an orientation vector to be defined, from the center of the body/tail cluster to the center of the head cluster (see Figure 5.5f), roughly estimating the cat's field of view. Moreover, not only the cats' body parts can be detected but also different body postures can be identified. Figure 5.6 shows six of the eight cats' postures described in Table 5.1 and their corresponding clustered image, i.e., with the cat's head, body and tail differentiated after running the k-means clustering algorithm over the depth data.
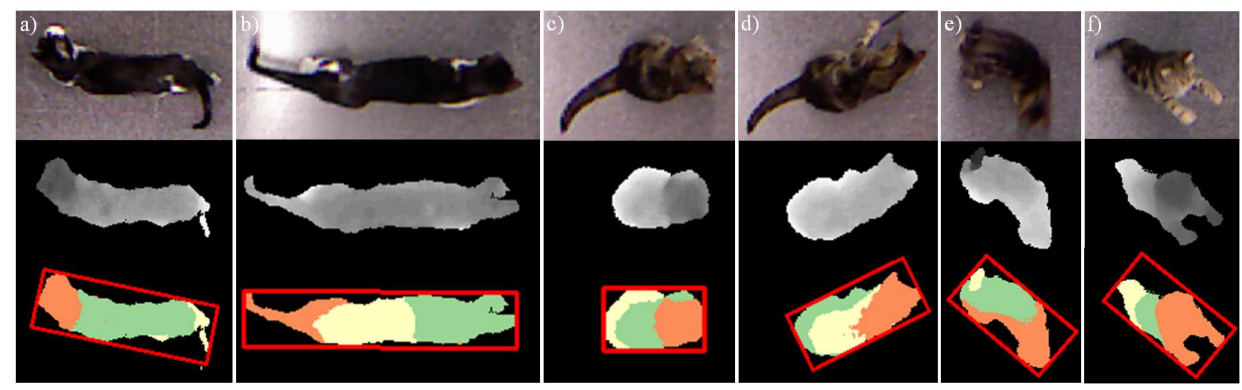

Figure 5.6: Color, depth and clustered frames for different cats' postures (a) standing (b) walking (c) sitting (d) semi-sitting (e) turning (f) jumping. 


\subsubsection{System Evaluation}

The process of matching cats' body parts to clusters and obtaining the posture of the animal can be performed in two different ways. On one hand, supervised training could be used to apply machine learning classifiers for the detection of different body postures and recognition of the cat's body parts. On the other hand, we could use a knowledge-based approach and classify the body parts and postures attending to the observed data set. Here we present an initial evaluation of both approaches for the classification of the cat's body parts into head, body and tail, as well as the identification of the following postures: standing, walking, sitting, semi-sitting, turning and jumping.

Supervised learning classifiers have been used for two different tasks. Firstly, to recognize both the cats' body parts, i.e., classify the detected clusters into head, body and tail, and secondly, to classify the cats' body postures. These two tasks have been evaluated independently. For the classification of cats' body parts, the labeled dataset contained a total of 4266 feature vectors. Each feature vector described a cat's body part, having three classes, i.e. head, body and tail, and therefore with 1422 feature vectors of each class. A feature vector describing a cat's body part was defined with the following features: width and height of the cat's contour, average depth, number of pixels and shape descriptors (second order moments and $\mathrm{Hu}$ invariant moments). Table 5.2 shows the accuracy results of a decision tree and a rule induction classifier using simple split validation $(70 \%$ of the data set used for training, stratified sampling). The rule induction classifier obtained better average performance, however the decision tree showed higher accuracy rates for the head and tail clusters. Both showed promising results and would allow to estimate the cat's orientation as for this it is only required to reliably classify the head and one of the two other body parts. For the classification of cats' body postures, 1422 manually labeled feature vectors were used. Each feature vector described a cat's posture, containing the following features: width and height of the cat's contour, clusters basic info (centroid, average depth, and number of pixels), distance between head to body centroids, distance between tail and body centroids, distance between head and tail centroids, angle between the vectors from body to tail and from body to head, depth differences between clusters (head and body, head and tail, body and tail), with a total of 21 features. Table 5.3 shows the accuracy results of a decision tree and a rule induction classifier using k-fold cross-validation (100 validations, stratified sampling) when classifying the aforementioned postures. Both classifiers performed relatively well, although accuracy rates vary among postures. However, the rule induction classifier performed better for almost all of the considered postures except for turning.

Supervised learning requires labeling the data, which is a time consuming task that not all users would be willing to perform. For example, it would be unreasonable to ask a cat owner to first train the system by labelling the data of her own cat. In order to allow the system to easily adapt to cats of different breeds, 
Table 5.2: Classification of cats' body parts using a supervised approach.

\begin{tabular}{c|cc}
\hline Body Parts & Decision Tree & Rule Induction \\
\hline Head & $96.64 \%$ & $84.63 \%$ \\
Body & $46.69 \%$ & $74.70 \%$ \\
Tail & $89.93 \%$ & $81.18 \%$ \\
Average & $61.58 \%$ & $82.20 \%$ \\
\hline
\end{tabular}

Table 5.3: Classification of cats' body postures using a supervised approach.

\begin{tabular}{c|cc}
\hline Postures & Decision Tree & Rule Induction \\
\hline Standing & $72.62 \%$ & $85.41 \%$ \\
Walking & $75.12 \%$ & $77.19 \%$ \\
Sitting & $89.77 \%$ & $92.90 \%$ \\
Semi-sitting & $81.15 \%$ & $84.78 \%$ \\
Turning & $81.40 \%$ & $80.19 \%$ \\
Jumping & $92.31 \%$ & $94.71 \%$ \\
Average & $79.56 \%$ & $85.50 \%$ \\
\hline
\end{tabular}

sizes and physical characteristics, it would be very beneficial if the tasks of labeling, training and deploying the model could be eased. Knowledge-based models have already been considered in several works $[167,360]$ when the costs for the preparation of datasets and training required in supervised learning are not feasible. Therefore, an exploratory study on the accuracy of the tracking system using knowledge-based classification has been conducted in order to assess the suitability of such an approach. First of all, an observational analysis on a sample of the recordings and their corresponding clustered images was conducted. As opposed to the supervised approach in which the classification of postures and body parts could be independent, within the knowledge-based approach the identification of each cluster depends on the posture being analyzed, and vice versa. For example, in some postures their clusters' characteristics were distinctive enough from

Table 5.4: Classification of cats' body parts and postures using a knowledge-based approach.

\begin{tabular}{c|cc}
\hline Postures & Posture Accuracy & Body Parts Accuracy \\
\hline Sitting/semi-sitting & $84 \%$ & $99 \%$ \\
Walking/Standing & $49.5 \%$ & $74.5 \%$ \\
Jumping & $69 \%$ & $92.5 \%$ \\
Turning & $53 \%$ & $71 \%$ \\
\hline
\end{tabular}


other postures and hence facilitated the classification, e.g., the average depth of the head's cluster is higher than in any other posture. In addition, the knowledgebased algorithm will not differentiate between walking and standing positions, nor between sitting and semi-sitting, as the differences between these postures are very subtle and better understood by existing machine learning algorithms. After the analysis, a customized decision tree algorithm was implemented which considered the following parameters: dimensions of the cat's contour, number of pixels for each cluster and average depth for each cluster. The decisions on the tree are made in terms of the observed average values for each feature on the data used for the analysis, allowing a threshold for the variance of each of these values. This classification algorithm has been evaluated for the sitting/semi-sitting, walking/standing, jumping and turning postures, as well as for classifying the different clusters in each posture into head, body and tail. Table 5.4 shows the result of the accuracy analysis of the knowledge-based approach conducted by extracting 200 random frames of each posture from the data set. Each of these frames was processed offline by the tracker and the knowledge-based classification algorithm. Regarding the posture, the results indicate the percentage of cases in which the decision tree assigns the correct label for the analyzed cat's contours. Regarding the body parts, the results indicate the percentage of cases in which the algorithm correctly identifies the head of the cat and its body and/or tail.

The results obtained using both supervised and knowledge-based approach are very promising and demonstrate that reliable depth-based tracking systems for animals can be developed for the classification of cat's body parts and postures within an interactive playful environment.

\subsection{Conclusions and Future Work}

This work has presented, to the best of our knowledge, the first observational study on cats' interactions and preferences towards different technological stimuli, as well as the first non-wearable tracking system capable of detecting different cats' postures, body parts, orientation and location of the animal during a playful scenario. With the promising results obtained, we are working towards the development of a fully functional interactive intelligent system which integrates the tracking system and the adaptation of the stimuli to the observed behaviors.

As future work, new observational studies with different devices and technological artifacts, either off-the-shelf or specifically designed ones, should be conducted. This would allow to assess whether different mechanisms provoke different interactions and behaviors, which should be included into the repertoire an intelligent playful environment would manage. 
Regarding the tracking system for the detection of cats' interactions, it remains to be studied whether just the detection of the aforementioned postures and movements would be enough to create engaging playful scenarios, or whether more postures should be detected. The detection of fine-grained interactions, such as touching a digital projection or tangible robot, or even more subtle cues as ear movements or tail postures, would not be feasible with the described system. Specific computer vision and machine learning algorithms should be implemented for this purpose. In all cases, the use of more accurate depth-sensors in this kind of tracking systems, such as the Microsoft Kinect ${ }^{\circledR}$ v2.0, would very likely improve the recognition accuracy rates. In addition, an exhaustive evaluation of the current system with different groups of machine learning classifiers, such as SVMs, Random Forests or kNNs among others, would be conducted. Combinations of several classifiers as well as feature selection mechanisms would also be considered.

The proposed approach for the detection of cats' postures, body parts, location and orientation could also be adapted to work with other animal species. A similar approach could be followed when working with species with similar behavior and anatomy, e.g. big felids, provided that the classification is adapted to the new dimensions and features of the species. In terms of species with different behaviors and/or anatomy, the tracking approach might change, and a prior observational study to assess the species and individual preferences and behaviors would be required. By conducting the presented behavioral study with cats, this paper provides an important step towards a formal definition of a methodology for the development of such interactive scenarios for animals. Moreover, the tracking system would also be useful for monitoring tasks in which the system could detect abnormal behaviors of the animals. It could also be used for training activities, in which the system would detect whether the animal is performing the specified posture correctly and provide a reward.

Humans should also be able to participate in this kind of playful scenarios, both physically and remotely. In this regard, it would be essential to study mechanisms to allow human participation in a seamless way. A similar tracking approach for the detection of human interactions could be incorporated into the system, as well as rules to allow natural interactions with the technological elements both for the animal and for the human player. The detection of the technological artifacts the system will manage could also be included as a feature of the tracking system. Together with the detection of the animals' posture and orientation, this information would allow to perform more accurate reactions.

Overall, this paper has presented promising mechanisms to lay foundations for the development of intelligent playful environments for animals, which would undoubtedly help to improve their wellbeing and quality of living by providing adaptive and stimulating interactive experiences. 


\section{Acknowledgments}

This work is funded by the European Development Regional Fund (EDRF-FEDER) and supported by Spanish MINECO (TIN2014-60077-R). The work of Patricia Pons is supported by a national grant from the Spanish MECD (FPU13/03831). Alejandro Catala received support from European Union's Horizon 2020 research and innovation programme under Marie Sklodowska-Curie grant agreement No 701991. 


\title{
Chapter 6
}

\section{Assessing machine learning classifiers for the detection of animals' behavior using depth-based tracking}

Patricia Pons ${ }^{1}$, Javier Jaen ${ }^{1}$, Alejandro Catala ${ }^{2}$

${ }^{1}$ ISSI Group, Department of Computer Systems and Computation (DSIC), Universitat Politècnica de València, Valencia, Spain

${ }^{2}$ Human Media Interaction, University of Twente, Enschede, The Netherlands

Published in Expert Systems with Applications 86, pp. 235-246, Elsevier (November 2017). doi:10.1016/j.eswa.2017.05.063

\begin{abstract}
There is growing interest in the automatic detection of animals' behaviors and body postures within the field of Animal Computer Interaction, and the benefits this could bring to animal welfare, enabling remote communication, welfare assessment, detection of behavioral patterns, interactive and adaptive systems, etc. Most of the works on animals' behavior recognition rely on wearable sensors to gather information about the animals' postures and movements, which are then processed using machine learning techniques. However, non-wearable mechanisms
\end{abstract}


such as depth-based tracking could also make use of machine learning techniques and classifiers for the automatic detection of animals' behavior. These systems also offer the advantage of working in set-ups in which wearable devices would be difficult to use. This paper presents a depth-based tracking system for the automatic detection of animals' postures and body parts, as well as an exhaustive evaluation on the performance of several classification algorithms based on both a supervised and a knowledge-based approach. The evaluation of the depth-based tracking system and the different classifiers shows that the system proposed is promising for advancing the research on animals' behavior recognition within and outside the field of Animal Computer Interaction.

\subsection{Introduction}

Technology is unquestionably changing our world and our lives every day. In this ever-growing digital era, human beings are not the only ones who can make use of technology. With an estimated 75 million households owning at least one pet animal in Europe ${ }^{1}$, and more than 71 million pet dogs and 73 million pet cats in $\mathrm{USA}^{2}$, animals are also a significant population coexisting with our technological surroundings. Domestic dogs and cats have been observed using some of our technological devices, such as smartphones or tablets, in their own way [16, 224, 351] and some zoos are also introducing touchscreens and tablet games for primate enrichment [41]. All these animals could benefit from the technological advances we have achieved throughout the digital revolution. However, animals have different physical features and mental perceptions of the world, preventing them from fully using and understanding our technology and interaction methods, which have been designed with human requirements and characteristics in mind.

Recently, spreading research is addressing the aforementioned concerns within the field of Animal-Computer Interaction (ACI) $[173,174]$. ACI considers animals as the target users of digital systems, and grounds on the development of computer interfaces and digital systems specifically designed for them. Animal-centered technology could improve animal welfare and wellbeing in several scenarios: interactive systems or devices could provide enrichment and stimulation for captive animals in zoos [41, 79], digital and/or tangible games could foster physical activity of animals in shelters and even entertain pets alone at home [113, 254], alleviating stress and isolation.

In the era of computers and with the advances in computer vision and machine learning techniques, a promising research line into ACI would be automatizing behavior recognition on animals. Animals are not verbal communicators; instead, they rely on body postures or sounds to express themselves. For these reasons,

\footnotetext{
${ }^{1}$ http://www.fediaf.org/who-we-are/facts-and-figures.html

${ }^{2}$ http://www.petfoodinstitute.org/?page=PetPopulation
} 
ACI studies are taking a big effort in developing technology for the automatic recognition of animal behavior and body postures. The benefits this technology could bring both to animal welfare and ACI research are countless. Firstly, it could provide both objective measurement mechanisms and more reliable feedback to inform the design and development of animal-centered technology. Second, animal welfare could be evaluated using automatic behavior recognition paired with machine learning techniques. This knowledge could be used to support the detection of abnormal behaviors of animals, allowing early detection of illnesses and other kind of problems which could be derived from an abnormal behavior. Finally, body posture and behavior recognition could also be used to automatically adapt the reactions of a system to the animals' interactions [254, 258]. In this way, animals could interact with systems on their own, improving animal wellbeing when they are alone (at home, zoos, shelters, etc.).

Great advances are being done using wearable devices as a way of recognizing animals' body postures and activities. These works make use of the information provided by accelerometers and gyroscopes attached to a wearable device such as a collar or harness that the animal has to put on. The extracted raw information from the sensors is then processed using machine learning techniques to train different classification algorithms to recognize animals' postures and activities. Depending on the activities and/or postures to be recognized, a wide range of classification algorithms have been used, either as standalone algorithms or combining them in a more complex learning process. However, there are animals who are not used to wear harnesses nor other wearable devices and could find those elements disturbing. Other animals, such as cats, have extreme agility and wide range of movements, and wearable devices could limit their naturalness. There are also animals to whom the use of wearable devices could pose a threat, such as wild animals, zoo animals or protected species. In addition, while wearable tracking systems might offer wider coverage area, they also require maintenance of batteries and sensors and one wearable device can only track one animal at a time. The use of non-wearable tracking mechanisms would be a promising complement for analyzing behavior and body postures in cases in which the use of wearable devices will not be feasible. These systems would allow more natural interactions within technologically-mediated environments as the animals will not be required to wear or carry any device, avoiding stress or affecting their behavior. Although the use of non-wearable systems would be delimited to a specific tracking area, they offer centralized maintenance and a single tracking device could provide information of several animals at a time. This information could also be used to train different classification algorithms for the automatic recognition of animals' postures, therefore providing the same benefits to ACI as the wearable approach in terms of animal wellbeing.

This paper describes the development of a non-wearable depth-based tracking system for cats and the promising results obtained by applying classification al- 
gorithms on the obtained depth information for the automatic recognition of the animals' body parts and postures. The use of depth information along with traditional computer vision techniques provides more information about the tracked animal than using solely an RGB camera. By exploiting this information using machine learning techniques and suitable classification algorithms, this approach could be a promising starting point towards the automatic detection and analysis of animals' behavior without requiring the animal to use any wearable device. This article is structured as follows: Section 6.2 analyzes previous tracking systems for animals based on wearable and non-wearable devices and their purposes, and states the necessity of a new approach grounding on previous research on non-wearable human-tracking systems. Section 6.3 describes the development of a depth-based tracking system for cats in indoor scenarios. Section 6.4 reports two experiments carried out to determine the accuracy of the system using both a supervised learning process and a knowledge-based approach for the classification of the cats' body postures and body parts, describing the different classification algorithms used and their accuracy rates. Section 6.5 explains how this tracking mechanism could be applied in several ACI domains and how it could be coupled with human and object tracking, and conclusions and future work are given in Section 6.6.

\subsection{Related Works}

This section provides an overview on existing research about animal tracking focused on animal gesture and body posture recognition for different purposes using both wearable and non-wearable tracking systems.

\subsubsection{Wearable Tracking Systems}

Several works have addressed the necessity of tracking animals in different scenarios. The most common method to gather information about the animal has been using wearable harnesses or collars with attached technological devices providing information to the system in charge of processing the information. One of the most basic methods for animal tracking in outdoor scenarios has been relying on GPS or radio-frequency localization, attaching the emitter devices to a collar or harness. These systems only give information on the animals' location and have been used by pet owners, mostly to assess their dogs' locations and whether or not they are in trouble [179]. This technology has also been used during hunting activities with dogs, allowing the human leading the hunting activity to interpret the movements of the dog in the field by following its signal on a handheld display $[235,344]$.

However, several outdoor scenarios require more precise information about the animals' movements or body postures during the activity, and even some kind of 
communication from the animal to the human side. As an example, determining the pose of the animal is of vital importance in the case of Search and Rescue (SAR) dogs. Due to its agility and strong sense of smell, dogs are suited to perform SAR tasks which are not always safe for humans, such as accessing small locations or identifying potential locations of survivors after a catastrophe. Usually SAR dogs have to work away from human sight, and it would be extremely useful for the dog handlers if they could know the location and pose of the dog to determine if the dog is trying to communicate some discovery, and to assess the physical wellbeing of the animal. Recognition of animals' postures and activities in this kind of scenarios are usually performed using accelerometers, gyroscopes or other inertial measurement units, and the majority of works are focused on dogs [30, 371]. In these works, differentiating clearly between what is activity and what is a posture is usually difficult. A specific activity entails that the animal adopts a specific posture, e.g. walking or jumping are different activities identified by their posture, and eventually some postures are in itself an activity, e.g. sitting.

Most of the works based on wearable devices for activity/posture recognition are based on the use of a tri-axial accelerometer located at the dog's collar and then apply classification techniques to the data obtained from the accelerometer in order to recognize the activity/posture. There are several devices for dogs, some of them even commercial, such as Whistle ${ }^{\circledR 3}$, FitBark ${ }^{\circledR} 4$ or WagTag ${ }^{\mathrm{TM}}$ [346] which make use of a tri-axial accelerometer to perform basic activity level recognition. However, these systems are only capable of indicating if the dog was resting or moving and do not differentiate between different activities which involve movement. In [158], dogs wear a tri-axial accelerometer on the collar and, after being trained with a kNN classifier, the system is able to differentiate between 14 activities and 2 postures.

Within the FIDO project [133], researchers have been studying how wearable devices could mediate the communication between working dogs and their handlers. They have undertaken extensive work on providing dogs with suitable wearable activators [132]. In addition, they have also considered to mediate this communication by recognizing motion-based dog gestures - sit, spin, roll, jump, etc. using a three-axis accelerometer attached to the front of a service dog harness [328]. More recently, they have studied the use of a dog collar with an accelerometer and gyroscope for the recognition of head gestures on dogs [330].

The effectiveness of wearable harnesses with several inertial measurement units located along the harness has also been studied. The work of Ribeiro et al. [274] uses the angles of two accelerometers on different locations on the dogs' harness to develop an algorithm capable of estimating four poses including: standing, lying down, sitting, and walking. Other works $[30,34]$ extend this idea by using

\footnotetext{
${ }^{3}$ http://www.whistle.com/

${ }^{4}$ www.fitbark.com
} 
more inertial measurement units located on the optimal locations of a dog's body, which have been determined attending to the algorithm's performance and the dogs' comfort and physiognomy. Using the information provided by these units and applying machine learning techniques, five static postures and three dynamic behaviors can be identified. They have also compared the performance of the classification algorithm using supervised against unsupervised classification methods [360].

Acceleration data-loggers are also a common and efficient way of detecting cats' body postures and frequent behaviors based on movement [340]. Commercial devices for cat activity recognition are also available, such as PawTrack ${ }^{\circledR} 5$, which detects whether the cat is at home or outside, and offers GPS geolocation for outdoor walks. However, it does not monitor any activity nor gesture. Cat@Log [367] is a non-commercial but more complete device. It consists of a cat collar device with several sensors: a camera, a GPS, an accelerometer, a Bluetooth module, battery and micro SD card. The camera provides videos of the cat's view, while the accelerometer data is used for activity recognition such as sleeping, jumping, walking or scratching.

Canine Amusement and Training [359] presents a wearable tracking system for dogs not based on accelerometers. It consists of IR emitters attached to the dog's harness, and a Wiimote's IR camera placed on the ceiling. The system detects the location and posture of the animal by tracking the IR emissions of the harness using the Wiimote. The detected postures and location are used by the system to determine whether the dog is performing correctly the proposed training activities offered by the system. Table 6.1 provides a summary of the existing approaches for wearable tracking systems for animals and their most distinctive features: the device being used for tracking, whether it works in indoor or outdoor locations, and whether or not it detects the position of the animal within the tracking area, its posture and/or its activity.

\subsubsection{Non-wearable Tracking Systems}

Although wearable devices are the most common way of tracking an animals' position and posture, a number of non-wearable solutions have also been proposed. Poultry.Internet [164] illustrates a remote communication system between a pet and its owner, in which the owner is able to remotely obtain real-time information on the location and orientation of its poultry inside the house backyard. This system tracks the movements of the chicken using a camera and an electro-pad located in the chicken's leg to sense its muscle activity. Through camera images they also detect the chicken's head to find the orientation of the animal within the backyard, but no postures are identified. In [148] computer vision methods have been used to track the movements of animals inside a zoo environment using

\footnotetext{
${ }^{5}$ http://pawtrack.com/
} 
Table 6.1: Existing wearable tracking systems for animals and summary of features.

\begin{tabular}{|c|c|c|c|c|c|}
\hline $\begin{array}{l}\text { Related } \\
\text { work }\end{array}$ & Device & $\begin{array}{l}\text { Indoor / } \\
\text { Outdoor } \\
\text { use } \\
\end{array}$ & $\begin{array}{l}\text { Position } \\
\text { detection }\end{array}$ & $\begin{array}{l}\text { Posture } \\
\text { recognition }\end{array}$ & $\begin{array}{l}\text { Activity } \\
\text { recognition }\end{array}$ \\
\hline $\begin{array}{l}\text { (Mancini et } \\
\text { al., 2012) [179] }\end{array}$ & Collar-worn GPS & Outdoor & Yes & No & No \\
\hline $\begin{array}{l}\text { (Paldanius et } \\
\text { al., 2011) [235] }\end{array}$ & Collar-worn GPS & Outdoor & Yes & No & No \\
\hline $\begin{array}{l}\text { (Weilenmann and } \\
\text { Juhlin, 2011) [344] }\end{array}$ & Collar-worn GPS & Outdoor & Yes & No & No \\
\hline Whistle & $\begin{array}{l}\text { Collar-worn GPS } \\
\text { and accelerometer }\end{array}$ & $\begin{array}{l}\text { Indoor and } \\
\text { outdoor }\end{array}$ & Yes & No & Basic \\
\hline FitBark & $\begin{array}{l}\text { Collar-worn } \\
\text { accelerometer }\end{array}$ & $\begin{array}{l}\text { Indoor and } \\
\text { outdoor }\end{array}$ & No & No & Basic \\
\hline WagTag [346] & $\begin{array}{l}\text { Collar-worn } \\
\text { accelerometer }\end{array}$ & $\begin{array}{l}\text { Indoor and } \\
\text { outdoor }\end{array}$ & No & No & Basic \\
\hline $\begin{array}{l}\text { (Ladha et } \\
\text { al., 2013) [158] }\end{array}$ & $\begin{array}{l}\text { Collar-worn } \\
\text { accelerometer }\end{array}$ & $\begin{array}{l}\text { Indoor (possibly outdoor } \\
\text { if the device range } \\
\text { of frequencies } \\
\text { allow it) }\end{array}$ & No & Yes & Yes \\
\hline (Valentin, 2014) [328] & $\begin{array}{l}\text { Accelerometer } \\
\text { on a harness }\end{array}$ & $\begin{array}{l}\text { Indoor and } \\
\text { outdoor }\end{array}$ & No & No & Yes \\
\hline $\begin{array}{l}\text { (Valentin et } \\
\text { al., 2015) [330] }\end{array}$ & $\begin{array}{l}\text { Collar-worn } \\
\text { accelerometer } \\
\text { and gyroscope }\end{array}$ & $\begin{array}{l}\text { Indoor and } \\
\text { outdoor }\end{array}$ & No & Yes & Yes \\
\hline $\begin{array}{l}\text { (Ribeiro et } \\
\text { al., 2009) [274] }\end{array}$ & $\begin{array}{l}\text { Two accelerometers } \\
\text { on a harness }\end{array}$ & $\begin{array}{l}\text { Indoor and } \\
\text { outdoor }\end{array}$ & No & Yes & No \\
\hline $\begin{array}{l}\text { (Bozkurt et } \\
\text { al., 2014 [30]; } \\
\text { Brugarolas et } \\
\text { al., 2013 [34]) }\end{array}$ & $\begin{array}{l}\text { Two accelerometers } \\
\text { and two gyroscopes } \\
\text { on a harness }\end{array}$ & $\begin{array}{l}\text { Indoor and } \\
\text { outdoor }\end{array}$ & No & Yes & Yes \\
\hline $\begin{array}{l}\text { (Watanabe et } \\
\text { al., 2005) [340] }\end{array}$ & $\begin{array}{l}\text { Two collar-worn } \\
\text { accelerometers }\end{array}$ & Indoor & No & Yes & Yes \\
\hline PawTrack & Collar-worn GPS & Outdoor & Yes & No & No \\
\hline Cat@Log $[367]$ & $\begin{array}{l}\text { Collar-worn GPS } \\
\text { and accelerometer }\end{array}$ & $\begin{array}{l}\text { Indoor and } \\
\text { outdoor }\end{array}$ & Yes & Yes & Yes \\
\hline $\begin{array}{l}\text { (Wingrave et } \\
\text { al., 2010) [359] }\end{array}$ & $\begin{array}{l}\text { Harness with IR } \\
\text { emitters and } \\
\text { Wiimote IR camera }\end{array}$ & Indoor & Yes & Yes & No \\
\hline
\end{tabular}

multiple cameras, but again no body postures nor gestures are identified. No wearable device is either used in Purrfect Crime [323], an interspecies digital game for cats and humans. In this case a Microsoft Kinect ${ }^{\circledR 6}$ is used to detect the position of the cats inside the play area, using the depth information provided by this sensor. However, the system only detects the central position of the animal, and no posture nor orientation are detected. Therefore, the interactive responses

\footnotetext{
${ }^{6}$ https://msdn.microsoft.com/en-us/library/hh855355.aspx
} 
of the system were sometimes erroneous, as not being able to identify where the cat was looking introduced some interactions which were not really intended by the animal.

To the best of the authors' knowledge, none of the existent non-wearable systems intended for animal tracking is capable of detecting body postures. In order to address the aforementioned limitations that wearable devices present in several application domains, there is a need for the development of tracking mechanisms for animals not based on wearable devices which allow to detect body postures as well as locating the animal within the tracked area. The following section describes a promising non-wearable tracking system for animals based on depth information. For the recognition of the animals' body parts and postures using the data provided by this depth-based tracking system, it has been essential to analyze how different classification algorithms perform in this domain. Both supervised and knowledge-based classification techniques have been tested, and the promising results obtained will be described in section 6.4 .

\subsection{Tracking System}

Within Human Computer Interaction, depth sensors have been successfully used for gesture detection and posture recognition on human beings. A depth frame of an image provides, for each pixel, the distance in millimeters from the sensor plane to the nearest object in that particular pixel. Depth sensors, such as the Microsoft Kinect ${ }^{\circledR}$, have been very useful to detect and recognize volumes and $3 \mathrm{D}$ shapes from $2 \mathrm{D}$ images. Usually, these sensors are located in a vertical plane, either in front of the user who is interacting or facing the scene to be analyzed. However, recent works have located this kind of sensors on the ceiling to track an open space area [23, 139, 140, 201], providing wider tracking areas and avoiding occlusion due to elements in the room. Human gestures can be detected using this set-up [20, 130, 168], and this configuration of the depth-sensor could also be useful for animal tracking applications. The skeleton of animals such as cats or dogs should be easily recognizable from above in several postures, e.g. sitting or walking, if volumetric information of the image such as depth analysis is used: the head could be identified as a volumetric shape different from the body and/or tail, and depth information would also allow to locate the position of each body part in a tridimensional representation space. In the case of other animals more similar to human beings in terms of skeletal characteristics, such as orangutans, similar approaches as the ones used for human gesture detection could be used. Therefore, a depth-based tracking system would be a promising way of detecting the animals' location, posture and field of view $[257,260]$. In the following sections, a depth-based tracking system for the detection of cats' body postures, location and orientation is described. The tracking system has been developed and tested 
with cats as target users, but it could be adapted to work with other animal species using a similar approach.

\subsubsection{Equipment and Procedure}

Several sessions with cats were carried out in which a Microsoft Kinect ${ }^{\circledR}$ v1.0 sensor was used to record video streams of depth and color information from the cats' natural movements during a period of time. During the sessions, cats moved freely and were also encouraged to play with their owners, caretakers or small interactive robotic toys. The Microsoft Kinect ${ }^{\circledR}$ was placed looking down from the ceiling at a height of $250 \mathrm{~cm}$, where it covered an area of approximately 200 $\mathrm{cm}$ long and $270 \mathrm{~cm}$ wide, as shown in Figure 6.1. 1. The tracking area was a clear space with no furniture nor objects besides the toys/robots used for the games. The Microsoft Kinect ${ }^{\circledR}$ recorded both color and depth video streams at a rate of 30 frames per second with $640 \times 480$ pixel resolution. Ethical guidelines for ACI studies were considered [325], and therefore the subjects were not forced to interact and they could walk freely around the room. Cats were encouraged to interact within the tracking area of the sensor by means of their owners/caretakers drawing their attention to this area with toys or by calling them. However, as the cat could move freely inside the room where the interaction took place, only the moments in which the cats were within the tracking area were valid recordings. These sessions allowed to obtain real data on common and spontaneous postures, behaviors and movements, which were later analyzed and processed to develop the depth-based tracking system. The tracking system has been developed using C\#, Microsoft Kinect ${ }^{\circledR}$ SDK 1.8, and EmguCV, an OpenCV framework for .NET systems which has been used for image processing.

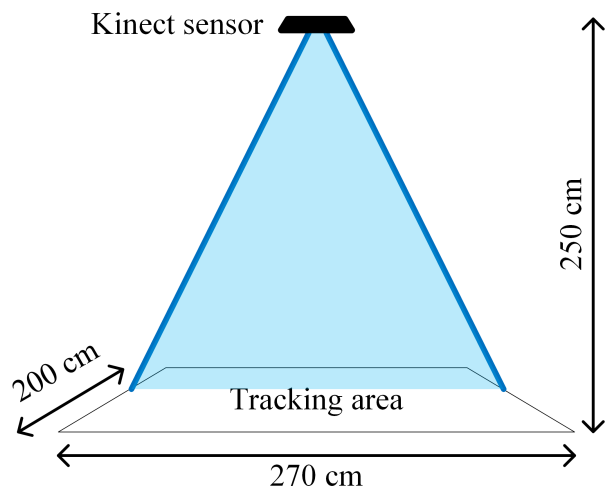

Figure 6.1: Set-up for the tracking system. 


\subsubsection{Processing Depth-based Information}

The Microsoft Kinect ${ }^{\circledR}$ v1.0 sensor provides both color (see Figure $6.2 \mathrm{a}$ ) and depth streams (see Figure 6.2b). The tracking system will only use depth information as input, so the color streams are discarded. Each depth frame provides, for each pixel, the distance in millimeters from the camera plane to the nearest object in that particular pixel (see Figure 6.2b). In the obtained depth images, the contours of the cats can be clearly observed as their depth values are greater than the floor's depth, which is constant. Instead, the depth pixels of a cat vary along its body, allowing a human eye to differentiate between the different parts of the cat's body just by looking at the depth frame. In the same way, different cat postures can be observed to generate different cat contours in the processed depth frames.

The first processing step of the algorithm consists of extracting the cat's pixels from the depth frame (see Figure 6.2b). In the current implementation of the algorithm, which has been tested with prerecorded data, background segmentation has been done by simply discarding from the image those pixels corresponding to the ground, as the sensor was placed in a fixed position during the sessions and therefore the distance to the floor was known (see Figure 6.2c). However, this approach can unintentionally remove the cats' tail (as observed in Figure $6.2 \mathrm{c}$, cat on the lower-right corner of the image), which in some cases could be a source of valuable information when creating richer posture descriptors. Thus, more elaborated approaches for background segmentation should be incorporated in order to overcome this current limitation and to allow for more flexible set-up conditions. With the floor removed from the image, computer vision algorithms are applied to the depth image in order to extract the cats' contours, which now appear as grey-scale blobs on the image (see Figure 6.2d). In this step, a cat's location within the tracked area can be determined by using the centroid of the extracted contours as a 2D coordinate. Each detected contour is then processed by a k-means clustering algorithm, which groups the pixels by their depth value and relative position (see Figure 6.2e): pixels of similar depth which are located together in the image would be grouped together within the same cluster. The number of clusters was set to three in order to divide the cat contour into the three most noticeable parts of the cat's body, i.e. head, body and tail. The following step of the tracking system would be the recognition of the cats' postures and body parts. For this purpose, the obtained clusters of each cat have to be classified into either head, body or tail. Once the head is detected, its position in relation to the body/tail clusters allows an orientation vector to be defined, from the center of the body/tail cluster to the center of the head cluster (see Figure 6.2f), roughly estimating the cat's field of view. Moreover, not only the cats' body parts can be detected but also different body postures can be identified. A pseudo-code description of the tracking, classification and clustering process is shown in the following algorithms. 

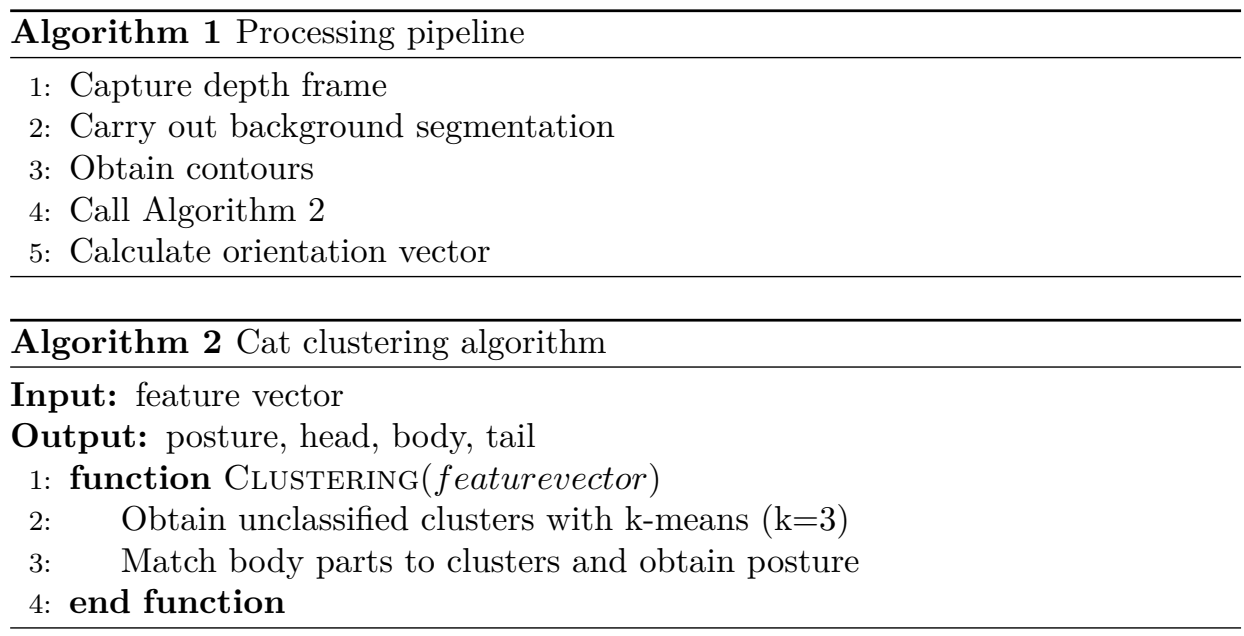

Step 3 in our clustering algorithm (Algorithm 2) can be carried out in different ways. In this work, a supervised training method and a knowledge-based classification system have been evaluated for the detection of both postures and body parts. Both approaches will be explained in sections 6.4.1 and 6.4.2 respectively. A dataset was created from the recordings in order to train and evaluate these classification mechanisms. It was observed that the tracking device was providing better images for one of the cats, probably due to its color, size and density of the coat. However, with the current data it was difficult to quantify to which extent each factor was affecting the tracking accuracy. To avoid introducing errors due to such factors in the experiment, only images in which the cat contour was correctly tracked and extracted were used for the experimental data. The dataset is comprised of 1422 contours of cats in different postures, which were manually labeled indicating the posture and the different body parts of the cat.

\subsection{Classification Results and Discussion}

A set of experiments have been conducted in order to determine the accuracy of the system in the detection of a cat's posture and the classification of its body parts. The following postures were considered, and representative color and clustered images of each of them can be observed in Figure 6.3:

- Standing: the cat is standing on its four legs, head slightly higher than the body

- Walking: the cat is standing, head slightly bent forward and legs moving forward 


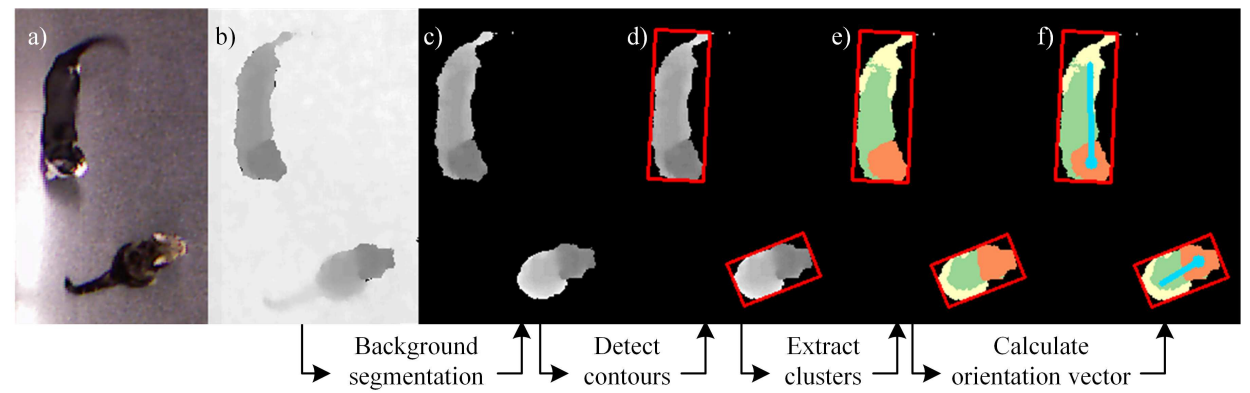

Figure 6.2: Process of extracting the cat's orientation: (a) color frame (b) depth frame, (c) background segmentation, (d) cat contours, (e) clusters for head, body and tail, (f) orientation vector.

- Sitting: the cat is sitting on its rear legs, without bending its front legs

- Turning: the head of the cat and its front part of the body are curving towards one side

- Semisitting: the cat is sitting on its rear legs but bending towards the front

- Jumping: the cat jumps on its back legs, head and front paws up, all body extended vertically

\subsubsection{Supervised Classification of Body Parts and Postures}

Supervised learning has been used to classify the three different body parts of a cat's contour into head, body and tail. The following features have been used to describe each body part: width and height of the cat's contour, average depth, number of pixels and shape descriptors (second order moments and $\mathrm{Hu}$ invariant moments). It should be noticed that the posture has not been considered a feature. In this way, the algorithm can firstly classify a cat's body parts regardless of its posture, and then use that information to properly create the feature vector of a cat's posture as will be explained later in this section. The labeled dataset of cats' body parts contained a total of 4266 feature vectors (1422 feature vectors of each class, i.e. head, body and tail) and the analysis was performed using the RapidMiner data mining tool. Table 6.2 shows the accuracy of several base classifiers tested using $\mathrm{k}$-fold cross validation (10 validations, stratified sampling) to analyze their performance, except for those marked with ${ }^{*}$, in which simple split validation was used ( $70 \%$ of the data set used for training, stratified sampling).

Results show rather low average accuracy rates. However, it can be seen that for several classification algorithms such as decision trees and random forest, very 
Table 6.2: Accuracy rates for base learners when classifying a cluster belonging to a cat's contour.

\begin{tabular}{c|cccccccc}
\hline & $\begin{array}{c}\text { Decision } \\
\text { tree }\end{array}$ & $\begin{array}{c}\text { Random } \\
\text { tree }\end{array}$ & $\begin{array}{c}\text { Random } \\
\text { forest }\end{array}$ & $\begin{array}{c}\text { Rule } \\
\text { induction }\end{array}$ & $\begin{array}{c}\text { Support } \\
\text { Vector } \\
\text { Machine }\end{array}$ & $\begin{array}{c}\text { kNN } \\
(\mathbf{k}=\mathbf{4})\end{array}$ & $\begin{array}{c}\text { Naïve } \\
\text { bayes }\end{array}$ & $\begin{array}{c}\text { Logistic } \\
\text { regression }\end{array}$ \\
\hline Head & $\mathbf{9 7 . 5 7 \%}$ & $42.98 \%$ & $77.55 \%$ & $84.63 \%$ & $64.90 \%$ & $61.46 \%$ & $34.16 \%$ & $49.23 \%$ \\
Body & $47.44 \%$ & $40.43 \%$ & $50.82 \%$ & $\mathbf{7 4 . 7 0 \%}$ & $59.37 \%$ & $54.58 \%$ & $29.63 \%$ & $59.34 \%$ \\
Tail & $\mathbf{9 3 . 0 0 \%}$ & $63.57 \%$ & $85.29 \%$ & $81.18 \%$ & $73.29 \%$ & $66.23 \%$ & $59.65 \%$ & $81.46 \%$ \\
Average & $62.87 \%$ & $47.17 \%$ & $62.28 \%$ & $\mathbf{8 2 . 2 0 \%}$ & $66.67 \%$ & $60.64 \%$ & $34.81 \%$ & $60.33 \%$ \\
\hline
\end{tabular}

promising accuracy rates are obtained when classifying the head and the tail. As we know that for each cat's contour, there is only one head, tail and body, the first two kinds of clusters could be identified using the learned classification model and the latter one, the body cluster, would be the remaining one. To demonstrate this hypothesis, a combined model has been built using a stacking approach and considering the three best base learning algorithms for this data: rule induction, support vector machine and decision tree. The resulting model, combined using rule induction, has an average accuracy of $83.18 \%$ (head $=88.22 \%$, body $=77.75 \%$, tail $=83.97 \%$ ). As expected, it follows the prediction if the output class is either head or tail, or classifies the cluster as body otherwise. With the head correctly classified, the field of view of the cat can be roughly estimated as an orientation vector from either the center of the body or tail to the center of the head. Supervised learning has also been used to classify a cat's posture. The same dataset of 1422 manually labeled images of cats in different postures was used. For each posture, the following features were considered: width and height of the cat's contour, clusters basic info (centroid, average depth, and number of pixels), distance between head to body centroids, distance between tail and body centroids, distance between head and tail centroids, angle between the vectors from body to tail and from body to head, depth differences between clusters (head and body, head and tail, body and tail), with a total of 21 features. Base learners performed as shown in Table 6.3 using k-fold cross-validation (100 validations, stratified sampling) when classifying the aforementioned postures. As can be observed in Table 6.3, rule induction was shown to be the best performing algorithm considering average accuracy scores. However, some algorithms perform better than others depending on the posture being classified. As an example, classification of jumping postures has slightly better results using a $\mathrm{kNN}$, random forest or Naïve Bayes classifier rather than rule induction.

Forward feature selection was applied on all base learners in order to discard features that could introduce noise into the classification. Accuracy results for the same cat postures are shown in Table 6.4. K-fold cross validation was used to analyze the performance of all classifiers (100 validations, stratified sampling), except for those marked with *, in which simple split validation was used ( $70 \%$ of the data set used for training, stratified sampling). It can be observed that in few 
Table 6.3: Accuracy rates for base learners when classifying a cat's posture.

\begin{tabular}{c|cccccccc}
\hline & $\begin{array}{c}\text { Decision } \\
\text { tree }\end{array}$ & $\begin{array}{c}\text { Random } \\
\text { tree }\end{array}$ & $\begin{array}{c}\text { Random } \\
\text { forest }\end{array}$ & $\begin{array}{c}\text { Rule } \\
\text { induction }\end{array}$ & $\begin{array}{c}\text { Support } \\
\text { Vector } \\
\text { Machine }\end{array}$ & $\begin{array}{c}\mathbf{k N N} \\
\mathbf{( k = 4 )}\end{array}$ & $\begin{array}{c}\text { Naïve } \\
\text { bayes }\end{array}$ & $\begin{array}{c}\text { Logistic } \\
\text { regression }\end{array}$ \\
\hline Standing & $72.62 \%$ & $50.65 \%$ & $55.17 \%$ & $\mathbf{8 5 . 4 1 \%}$ & $83.44 \%$ & $85.32 \%$ & $84.62 \%$ & $84.62 \%$ \\
Walking & $75.12 \%$ & $51.35 \%$ & $76.39 \%$ & $\mathbf{7 7 . 1 9 \%}$ & $74.89 \%$ & $74.55 \%$ & $63.31 \%$ & $71.43 \%$ \\
Sitting & $89.77 \%$ & $67.27 \%$ & $85.71 \%$ & $\mathbf{9 2 . 9 0 \%}$ & $89.08 \%$ & $87.63 \%$ & $90.86 \%$ & $90.00 \%$ \\
Semisitting & $81.15 \%$ & $58.76 \%$ & $87.25 \%$ & $84.78 \%$ & $78.69 \%$ & $\mathbf{8 6 . 5 7 \%}$ & $81.02 \%$ & $78.12 \%$ \\
Turning & $\mathbf{8 1 . 4 0 \%}$ & $42.07 \%$ & $79.35 \%$ & $80.19 \%$ & $70.98 \%$ & $71.68 \%$ & $62.17 \%$ & $73.78 \%$ \\
Jumping & $92.31 \%$ & $80.47 \%$ & $96.84 \%$ & $94.71 \%$ & $93.66 \%$ & $96.69 \%$ & $\mathbf{9 6 . 9 7 \%}$ & $92.92 \%$ \\
Average & $79.56 \%$ & $55.84 \%$ & $69.59 \%$ & $\mathbf{8 5 . 5 0 \%}$ & $81.93 \%$ & $83.32 \%$ & $79.01 \%$ & $82.05 \%$ \\
\hline
\end{tabular}

cases the average and individual accuracy rates are significantly improved, such as in the random tree, random forest and $\mathrm{kNN}$ classification algorithms. The selected features are different depending on the algorithm being used, hence it seems that there are no noisy features and the best performing subset of features will depend on the algorithm being used. With this approach, the kNN classifier not only has the best average performance score, but also is the best classifier for four of the six postures, and in the remaining two postures it is the second best performing classifier.

Table 6.4: Accuracy rates for base learner when classifying a cat's posture, using forward feature selection.

\begin{tabular}{c|cccccccc}
\hline & $\begin{array}{c}\text { Decision } \\
\text { tree }\end{array}$ & $\begin{array}{c}\text { Random } \\
\text { tree }\end{array}$ & $\begin{array}{c}\text { Random } \\
\text { forest }\end{array}$ & $\begin{array}{c}\text { Rule } \\
\text { induction* }\end{array}$ & $\begin{array}{c}\text { Support } \\
\text { Vector } \\
\text { Machine* }\end{array}$ & $\begin{array}{c}\text { kNN } \\
(\mathbf{k = 4 )}\end{array}$ & $\begin{array}{c}\text { Naïve } \\
\text { bayes }\end{array}$ & $\begin{array}{c}\text { Logistic } \\
\text { regression* }\end{array}$ \\
\hline Standing & $76.05 \%$ & $63.06 \%$ & $74.64 \%$ & $88.64 \%$ & $85.92 \%$ & $\mathbf{9 2 . 9 8 \%}$ & $82.77 \%$ & $73.81 \%$ \\
Walking & $79.27 \%$ & $59.56 \%$ & $71.94 \%$ & $80.60 \%$ & $69.70 \%$ & $\mathbf{8 7 . 8 3 \%}$ & $77.92 \%$ & $79.03 \%$ \\
Sitting & $91.33 \%$ & $87.80 \%$ & $90.75 \%$ & $94.12 \%$ & $83.61 \%$ & $94.12 \%$ & $\mathbf{9 6 . 6 4 \%}$ & $88.89 \%$ \\
Semisitting & $80.45 \%$ & $74.80 \%$ & $78.86 \%$ & $82.61 \%$ & $\mathbf{1 0 0 . 0 0 \%}$ & $84.67 \%$ & $82.19 \%$ & $77.50 \%$ \\
Turning & $74.57 \%$ & $62.42 \%$ & $73.33 \%$ & $76.81 \%$ & $74.24 \%$ & $\mathbf{8 7 . 3 2 \%}$ & $71.22 \%$ & $83.78 \%$ \\
Jumping & $93.56 \%$ & $92.57 \%$ & $97.01 \%$ & $96.72 \%$ & $96.61 \%$ & $\mathbf{9 7 . 5 2 \%}$ & $96.52 \%$ & $89.23 \%$ \\
Average & $81.10 \%$ & $70.74 \%$ & $79.58 \%$ & $86.62 \%$ & $83.80 \%$ & $\mathbf{9 1 . 2 2 \%}$ & $83.63 \%$ & $80.05 \%$ \\
\hline
\end{tabular}

As a final validation, a combined model has been trained using a stacking approach, considering the three best performer classifiers $(\mathrm{kNN}$, rule induction and support vector machine) and combining them using rule induction. Forward feature selection has been applied to the combined model. The average accuracy for this model is $88.50 \%$, with the following class accuracy: standing $=87.32 \%$, walking $=80.56 \%$, sitting $=94.00 \%$, semisitting $=86.36 \%$, turning $=89.47 \%$, jumping $=96.72 \%$.

The obtained results are very promising and demonstrate that reliable tracking systems for animals based on depth information can be developed using machine learning algorithms for the classification of the cat's body parts and postures. 


\subsubsection{Knowledge-based Classification of Body Parts and Postures}

The process for supervised learning is time demanding as it requires to label the data in order to construct the training set and build a suitable model to apply to the new data. As this system is envisioned to adapt to cats of different breeds, sizes and physical characteristics, it would be very beneficial if the tasks of labeling, training and deploying the model could be eased. Other works have also considered including knowledge-based models directly encoded in classification algorithms for solving several problems $[167,360]$, when the costs for the preparation of datasets and training required in supervised learning are not feasible. Therefore, an exploratory study on the accuracy of the tracking system using knowledgebased classification has been conducted in order to assess the suitability of such an approach. If feasible, it could allow easy deployment and adaptation of the system to new scenarios and users, i.e. cats of different sizes and breeds, with minimal configuration requirements.

In order to develop such a system, an observational analysis on a sample of the recordings and their corresponding clustered images was conducted. Different cat postures were seen to generate different cat contours in the processed depth frames, and the sizes and depth values of the different clusters also varied from one posture to another. As an example, the cat's depth stream contour when sitting showed a smaller, square-shaped bounding rectangle (see Figure 6.3c), while the depth stream when standing or walking revealed a larger and rectangular-shaped bounding rectangle (see Figure 6.3a and Figure 6.3b). In addition, the pixels of the sitting cat's head had a significantly higher average depth than the pixels of the rest of the body. This is observed in Figure $6.3 \mathrm{c}$, in which the grey pixels of the head are significantly darker than the pixels of the cat's haunch. In contrast, the cat walking in Figure 6.3b has an average depth of its head pixels very similar to the average depth of the haunch and tail pixels. Another aspect to consider is that the k-means clustering algorithm has been fixed to provide three clusters at all times. Hence, the classification algorithm for the different body parts is the one responsible of determining which one would correspond to the lower back of the cat. This cluster, in most cases, corresponds to the tail. However, in some cases in which the tail is not visible such as in Figure $6.3 \mathrm{c}$, the detected cluster physically corresponds to the lower back of the animal. These cases usually occur when the cat is in a sitting posture. In this case, the head is clearly differentiated from the rest of the body, and in terms of average depth it is undoubtedly the highest cluster detected. Hence, in this posture only the detection of the head and the body would allow the cat's field of view to be determined. However, in other postures the detection of the tail is crucial to determine the orientation of the cat. For instance, determining whether the cat is looking north or south in Figure 6.3b would be difficult if the tail was not detected, because the head is not clearly in a higher position than the rest of the body. 

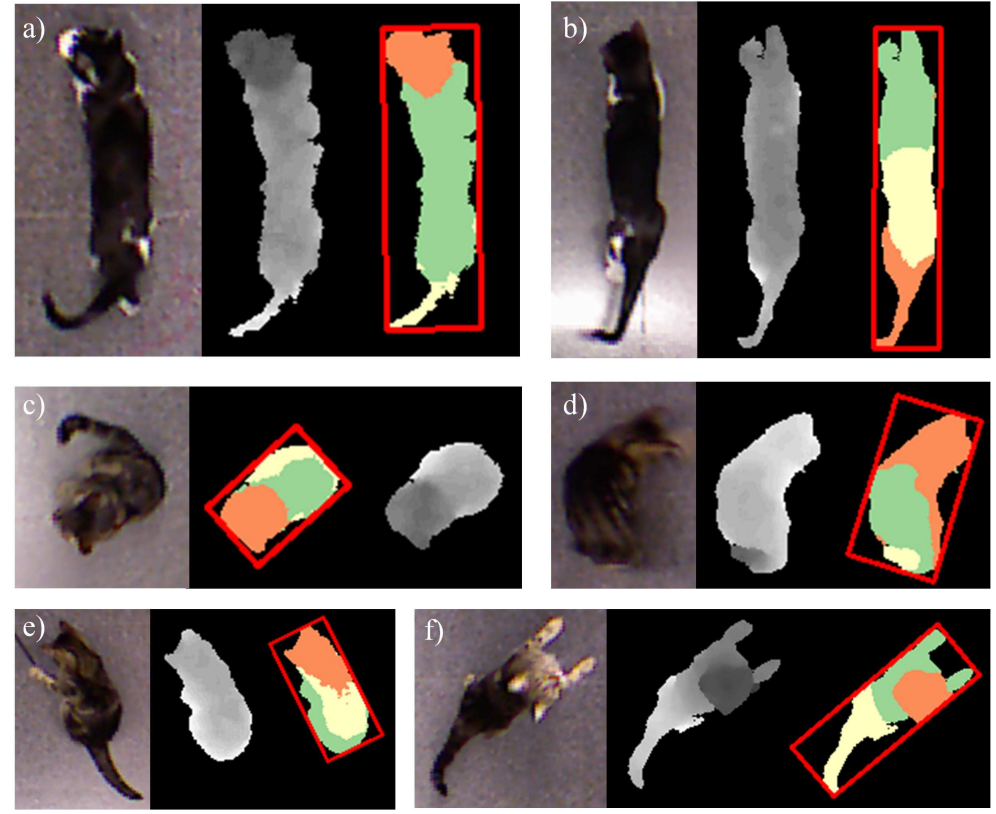

Figure 6.3: Cat's postures and the corresponding depth and clustered image (a) standing (b) walking (c) sitting (d) turning (e) semisitting (f) jumping.

After the analysis, a decision tree algorithm was developed which considered the following parameters: dimensions of the cat's contour, number of pixels for each cluster and average depth for each cluster. The decisions on the tree are made in terms of the observed average values for each feature on the data used for the analysis, allowing a threshold for the variance of each of these values. Several simplifications have been made. Firstly, the algorithm cannot differentiate between walking and standing positions, nor between sitting and semi-sitting, as considering this basic information the average and threshold values overlap and it is only possible for machine learning algorithms to build such an elaborate classification. Therefore, this basic version of the tracking system is focused on detecting sitting/semi-sitting, walking/standing, jumping and turning positions of several cats at a time, and classifying the different pixel regions in each posture to detect the head, body and tail of each cat. Secondly, with the supervised approach it was possible to isolate the classification of postures from the classification of clusters into cats' body parts. It is not possible to do that in this knowledge-based approach, as for the human observer the identification of each cluster depends on the posture being analyzed, and vice versa. Third, and extra piece of information has been given to the algorithm. If the decision tree cannot find the head of the cat, information from the last previous frame will be used to find the closest cluster 
Table 6.5: Accuracy of the tracking system identifying the cats' postures and body parts.

\begin{tabular}{c|cccc}
\hline & Sitting / semisitting & Walking / standing & Jumping & Turning \\
\hline Posture & $84 \%$ & $49.5 \%$ & $69 \%$ & $53 \%$ \\
Body parts & $99 \%$ & $74.5 \%$ & $92.5 \%$ & $71 \%$ \\
\hline
\end{tabular}

which was classified as the head in the last frame. The pseudocode in Algorithm 3 shows the basic behavior of the decision tree algorithm.

An accuracy analysis has been conducted extracting 200 random frames of each posture and processing them offline by the tracking and classification algorithms. Table 6.5 shows a summary of the results indicating the percentage of frames for each posture in which the algorithm correctly identified the posture and body parts of the cats respectively. Regarding the posture, the results indicate the percentage of cases in which the decision tree assigns the correct label for the analyzed cat's contours. Regarding the body parts, the results indicate the percentage of cases in which the algorithm correctly identifies the head of the cat and its body and/or tail.

As it can be observed, the sitting position is one of the most distinctive ones as it usually comprises a small area in which the head can be clearly identified and hence the body parts are very easily classified and the orientation vector between the head and body/tail can be almost perfectly traced. The algorithm also classifies very well the cat's body parts when jumping, although the classification of this posture has a $31 \%$ error rate. This is mostly due to the moments in which the jump is starting or finishing. In these cases, the threshold value established to determine the jump, which is the difference in depth between the highest and the deepest cluster, does not fit well. This accuracy rate could probably be improved by adjusting more carefully this threshold. The algorithm had problems identifying the walking/standing position as well as the turning one. Nevertheless, in both cases the classification of the body parts offers promising accuracy rates and allows to determine an orientation vector to estimate the animal's field of view.

At this stage the knowledge-based classification algorithm would not be suitable to provide very accurate posture classification in all cases. It is likely that the decisions coded in the algorithm do not represent well the most characteristic features of each postures as it has been a manual process based on observation and codification. This is an issue that supervised classification algorithms are capable of resolve, as it has been demonstrated by the results in section 6.4.1. However, the knowledge-based classification algorithm presented in this section performs well in terms of body parts classification, allowing to determine the different body parts of the animal correctly with less training time than in a supervised approach. 


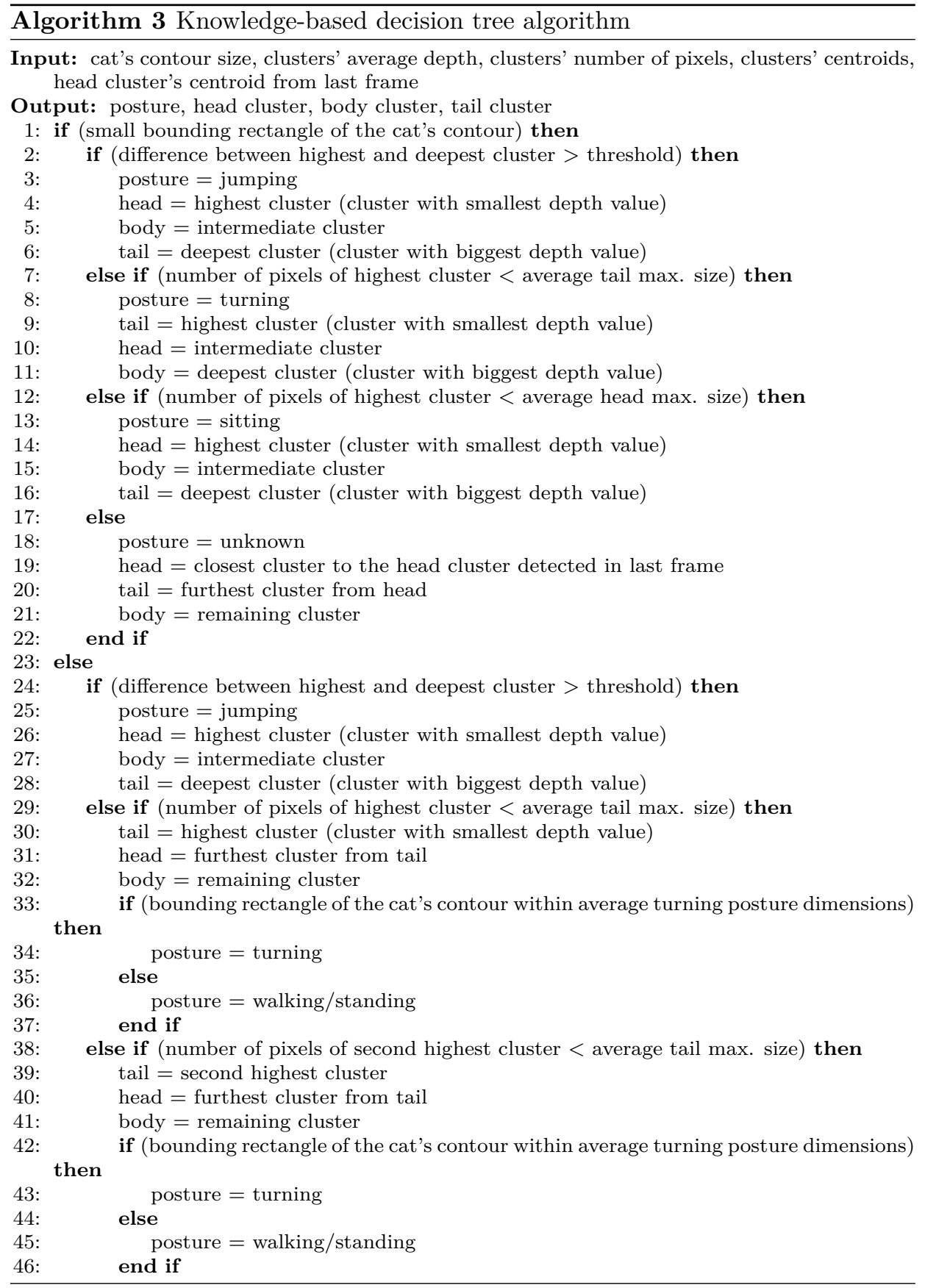




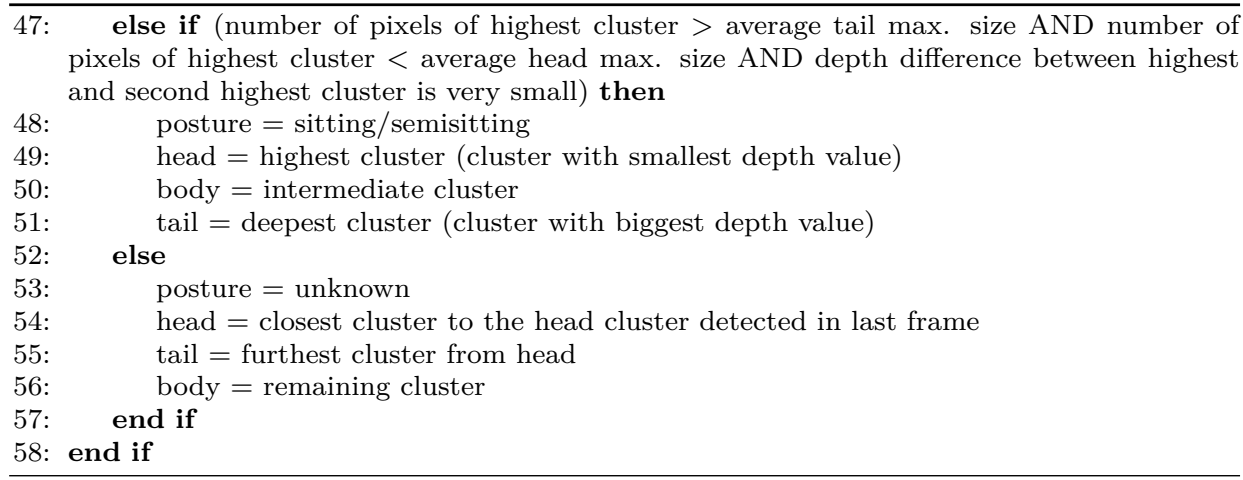

Therefore, for a system to automatically recognize between different body postures of an animal with very high certainty, supervised classification algorithms are preferred over a basic knowledge-based approach. If more complex systems are to be developed, supervised classification algorithms are the recommended approach. For example, they could provide very reliable information in monitoring systems for animal welfare and behavioral pattern recognition, or remote communication systems for dogs with occupations based on postures. Nevertheless, a knowledgebased classification technique could provide satisfactory accuracy rates if other types of information derived from the body parts of the animal are required, such as determining its field of view or just detecting a specific part of the animal such as the head. The next section will provide an overview of the different systems in which classification algorithms as the ones presented would be a key component.

\subsection{Applications within Animal-Computer Interaction}

\subsubsection{Behavior Recognition, Learning Behavioral Habits and Welfare Assessment}

Non-wearable depth-tracking systems as the one presented in this paper would significantly contribute to the development of ACI research. ACI studies usually rely on the observational clues and expert knowledge provided by pet owners, caretakers, zookeepers or specialists in animal behavior. They can provide insightful interpretations about an animal's body language by analyzing its posture and movements, as understanding and analyzing the animals' body language is one of the potential ways in which ACI researchers can interpret the animals' reactions to a system. Therefore, a tracking system capable of detecting body postures, together with expert knowledge on animal behavior, could have the potential to automatize the interpretation of an animal's responses to a digital system. Wear- 
able systems might not be used in all scenarios; hence, non-wearable technologies would provide great advantages in this regard.

One of the main benefits of not using wearable devices would be the possibility of creating systems capable of constantly monitoring an animal's activity without disturbing it nor modifying its habits. Complete animal tracking systems such as the one presented in this paper coupled with machine learning algorithms would allow us to learn behaviors and habits of individual animals during their usual daily life. In this way, a personalized knowledge base could be obtained for each animal, similar to the knowledge their human companions have about them. Through the tracking system we could learn, for example, the habitual location and movements an animal performs in a specific context, the amount of time a day it spends doing physical activity such as walking or playing, and the intensity of this activity. This knowledge base could be used to detect changes in behavioral patterns, such as increasing/decreasing physical activity or resting time, therefore supporting early detection of illnesses or other problems.

Zookeepers could make use of these systems in order to assess the wellbeing of animals in zoos by means of detecting abnormal behavioral patterns. Automatically detecting behaviors of animals in shelters could also alert the caretakers in case that abnormal activity patterns are detected on an animal, allowing the caretakers to take rapid action to foster the animal's physical activity, dedicate more time to that animal or prioritizing adoption for cases in which the animal does not respond to those cares. In addition, this automatic behavior detection could allow to create personalized profiles for each animal. When adopting, it is very important that the personality of the animal matches the needs and personality of the human beings who are adopting it. In this way, profiles of the animals' personality could be extracted and presented to the adopters [4]. Finally, pet owners could also use this kind of systems in order to detect whether their animals are doing well while left alone at home, for example during working hours, and communicate remotely with them via cameras and microphones if abnormal behaviors are detected.

\subsubsection{Playful Environments based on Gesture and Posture Recognition}

Tracking systems such as the one presented in this paper have an essential role in the development of future playful environments for animals. Current digital games for animals have been usually tied to a specific device, human participation has usually been limited to a "controller" or "assistant" role and there has been no support for several animal participants playing together as differentiated players [258]. However, traditional games with animals rely on a more natural and open interaction, meaning that both animals and humans can move freely during the game. Animals are used to playing by themselves or with humans, and in the 
latter case the human is an active and essential participant in the activity. In addition, traditional games make use of the elements in the environment to enhance the playful experience, not limiting it to the object itself but to the spontaneous interactions between the players thanks to the mediating object.

Future technologically-mediated games for animals could therefore be conceived as multimodal and multi-device systems, in which animals could play either alone, in a group or with human beings in a natural way. If animals play by themselves, the system should intelligently manage the different devices and objects in the environment in order to adapt the game to the animals' preferences and interactions $[254,258]$. In order to keep the animals' interest in the game, some kind of "intelligence" is required. Random movements or actions of a digital element would cause the animal to eventually lose interest in the activity [253, 260]. Hence, being able to interpret an animal's body posture and interactions in a similar manner humans do is very important for providing proper actuations in the playful environment. A tracking system capable of detecting a cat's location, posture and orientation coupled with the detection of digital objects and their movements would allow us to create engaging and realistic games using technological artifacts which adapt to the detected cat's postures and field of view. As an example, we could think of a game in which a cat chases a Sphero ${ }^{\circledR}$ (electronic ball, see Figure 6.4) controlled by the system, but the movements of the ball are not random. Instead, the Sphero ${ }^{\circledR}$ could also be tracked by the system and programmed to move depending on the detected context. Detecting the location of the cat inside the play area would allow the game to start whenever the cat approached the electronic ball. The detected cat's field of view could be used as the region on which to deploy and move the technological artifacts to attract and maintain its attention. Posture detection would allow the system to move away the toy if the tracking system detects that, for example, the cat is crouched in a hunting position, waiting for the ball to approach. The presented tracking system would have other advantages in this regard, as not all animals perform the same interactions in play: during a chasing game, a cat might prefer to wait patiently until an object approaches him to catch it, whereas another cat might be more eager and prefers to run after the object. Therefore, the tracking system could help to learn the specific play dynamics and preferences of an animal during the game in order to adapt it.

If human beings are also participating, their interactions should also be considered an essential part of the game and the digital playful experience should be as natural as traditional games. The automatic recognition of human gestures by a top-down depth-based tracking system would allow humans to participate in the game in a natural way for both the animal and the human, not being tied to any specific device. Human gestures could be used to control the movements or features of the digital elements in the play area, e.g. the human player points at an element with his hand and then points to another place, and the element moves in the direction indicated by the human. Some games could introduce spe- 




Figure 6.4: Kitten chasing a Sphero ${ }^{\circledR}$.

cific gesture-based interactions, e.g. a competitive game between the animal and the human in which a clapping gesture makes the system move a toy/cord for 10 seconds to distract the cat so the human can take advantage.

\subsubsection{Tracking Systems for Zoo Enrichment and Open Spaces}

Depth sensors for animal tracking are a powerful tool in order to help zoos improve their current enrichment practices. Technology is being integrated into enrichment activities at zoos worldwide, as it easily allows creating varied scenarios and cognitive stimulation for the animal [80]. In these environments, animals are not allowed to acquire and hold the technology themselves. Therefore, zoo enrichment activities based on technology would benefit from mechanisms that track and augment the animals' interactions with other elements in their environment. In this way, all the technological devices could be placed outside the enclosure while the animal interacts naturally with the augmented elements inside [252]. This also leverages the need of a human caretaker being present to provide the technology. For example, current studies include the use of depth sensors to detect the interactions of orangutans with a projection on the floor [343]. Tangible elements inside the environment have also been augmented using RGB-D sensors. By tracking and detecting non-technological tangible objects, the system could produce responses such as sounds based on the animals' movements with those objects [247].

However, due to current limitations of the RGB-D technology, the tracking system described in this paper is only suitable for indoor detection. Other methods would have to be considered if we wanted to carry out animal tracking outdoors 
without using a wearable device, such as dogs in their yards or wild animals in their habitats. In these scenarios, lighting conditions and required distance for optimal tracking would make the acquisition of accurate information from the depth sensor very difficult. In fact, current works for outdoor animal tracking usually rely on computer vision techniques either from recorded videos from regular video cameras [223], heat-cameras [336] or drone mounted cameras for survey population [122]. The use of drones for posture and behavioral tracking seems promising but still presents many issues that could disturb the animals' natural behaviors: distance from the device to the animal, noise of the device, battery life, transmission rate and range, etc. Although this is an interesting area, it requires deeper discussion and consideration.

\subsection{Conclusions and Future Work}

A tracking system for cats based on depth information has been developed, and two studies comparing its performance using both supervised and knowledge-based approaches for the classification of cat's postures and body parts have been presented. Results have shown to be very promising and therefore tracking systems for cats based on depth information could effectively detect a cat's location and also use classification algorithms to recognize a cat's postures and body parts. Several applications have been envisioned for this kind of tracking systems and its benefits for animal welfare and wellbeing have been outlined.

Our future work would be improving the tracking and classification system, for example by introducing temporal information which could help to better differentiate between several postures such as walking or standing, and allowing to register paths and sequences of movements which are usually performed together. New postures and behaviors could also be identified, and new experiments should be conducted with cats of different sizes and breeds in order to contrast the results. In this regard, improving the knowledge-based classification process could allow to achieve fully adaptation of the system to different cats. For example, the tracking system could automatically detect the size of the cat, in case it is a kitten, and adapt the classifier to it without requiring the researcher or owner of the animal to perform manual labeling of the data to train a new classifier, which is a very time consuming task.

Some improvements could also be done regarding the detection of the cat's field of view. The orientation vector defined as a vector from the body/tail of the cat to its head sometimes gives misleading information when the cat is turning its head around. In these scenarios, the orientation vector might not be accurate when determining the cat's head orientation. Computer vision could then be used to recognize the shape of the cat's head and detect characteristic features, such as nose or ears. This would allow the detection of the head orientation and hence 
correctly determining where the cat is looking at. Cats have a field of view of $200^{\circ}$; therefore detecting the head orientation would be sufficient to provide a broad area of around $100^{\circ}$ centered using the head orientation vector, in which to locate the technological intervention.

Another feature that will greatly benefit the tracking system in terms of flexibility and adaptation is automatic background segmentation. The tracking system presented in this manuscript performed background segmentation assuming a fixed position from the sensor to the floor, and within a clear space with no obstacles nor furniture. However, from the initial frames of the tracking area without any animals or humans in it, background segmentation could be applied to detect the floor (i.e., deepest area in the image). Once the floor has been extracted from the image, static objects with higher depth than the floor could be detected. This would correspond to furniture, obstacles, etc. Once the area and obstacles have been mapped, any new information with different depth than the one recorded in the initial static frames would correspond to the player/s. This process would allow the tracker to adapt to several scenario configurations without minor interventions from the human, either researcher or owner/caretaker.

It should also be noted that for this study, the depth sensor was a Microsoft Kinect $^{\circledR}$ v1. It is known that the newer version of this sensor provides better tracking accuracy. Hence, it remains to be studied whether this new sensor would help to improve the results presented in this paper. Moreover, it could also help to quantify and reduce tracking issues caused by the physical characteristics of the cats, e.g. color, size, density of the coat, etc. In addition, it would be very interesting to evaluate the tracking system with other animal species, not only four legged ones, such as dogs, but also animals with very different skeletal characteristics such as orangutans. The next step would be the integration of the tracking system and the learned classifiers into an intelligent playful environment, as explained in section 6.5.2. In this way, the system could react properly according to the detected behavior, creating engaging playful scenarios for the animal who is playing. The proposed tracking approach for animals based on depth information as well as the two studies on the performance of different classifiers in this domain are a significant and beneficial contribution for advancing research within and outside the field of Animal Computer Interaction. The outcomes of these two studies will allow to improve the techniques for posture and behavior recognition of animals using non-wearable devices, which will be used in the development of systems to support animal welfare and improve animal wellbeing. 


\section{Acknowledgements}

This work is funded by the European Development Regional Fund (EDRF-FEDER) and supported by Spanish MINECO with Project TIN2014-60077-R. It also received support from a postdoctoral fellowship within the VALi+d Program of the Conselleria d'Educació, Cultura I Esport (Generalitat Valenciana) awarded to Alejandro Catalá (APOSTD/2013/013). The work of Patricia Pons is supported by a national grant from the Spanish MECD (FPU13/03831). Special thanks to our cat participants and their owners, and many thanks to our feline caretakers and therapists, Olga, Asier and Julia, for their valuable collaboration and their dedication to animal wellbeing. 



\section{Part III}

\section{Tangible User Interfaces for Animal Enrichment}



The non-wearable tracking system approach described in Part II shows promise for advancing research on the recognition of animal behavior, and led to the exploration of this NUI approach for the detection of other animal species. As animals of different species behave differently and also have different physiological characteristics, the ways in which a system extracts information from their behavior would also differ. This part of the thesis therefore studies the application of the proposed technological knowledge to create interactive spaces for different animal species in a different context, i.e. orangutans in a zoo environment.

For this purpose, an initial design and study of the environment and species was required in order to design a suitable NUI capable of adapting to the speciesspecific behavior, anatomy, and location. This in turn led to the proposal and design of tangible user interfaces for orangutans that would allow the animals to manipulate everyday tangible objects from their environment in order to trigger reactions from the system. A non-wearable tracking system was therefore designed and developed to recognize the orangutans' spontaneous interactions with such objects in order to create an animal-centered enrichment experience for them.

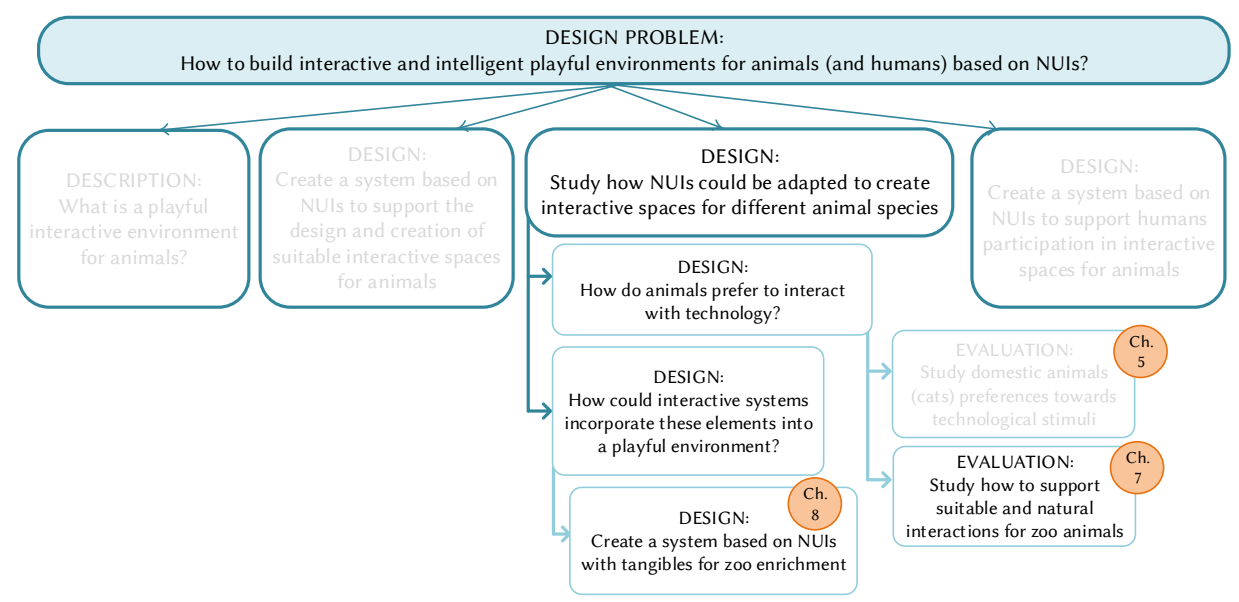

Figure 6.5: Problem decomposition of the work described in Part III. 



\title{
Chapter 7
}

\section{Tangible User Interfaces for Zoo Enrichment}

\author{
Patricia Pons, Javier Jaen
}

ISSI Group, Department of Computer Systems and Computation (DSIC), Universitat Politècnica de València, Valencia, Spain

Published in Proceedings of the 2016 CHI Conference on Human Factors in Computing Systems Workshops - HCI goes to the Zoo, pp. 1-4, (2015).

\begin{abstract}
The design of technological enrichment scenarios in zoo enclosures is usually limited by the kind of devices and technologies which can be used in order to preserve the animals' welfare. Due to the animals' strength and spontaneity, technological devices could be broken, swallowed or cause harm to the animal if manipulated improperly. However, there are animals with innate dexterity in the manipulation of tools and objects, such as orangutans. Instead of providing enrichment to these animals using technological objects outside the enclosure and under the supervision of zoo keepers, we propose to explore the potential of using non-technological tangible objects for primates as tools to interact within an interactive space. These interactive spaces could be shared with zoo visitors, grounding on the commonalities of humans and primates in terms of object manipulation to create both an enriching experience for the primate and an educational activity for the zoo visitor.
\end{abstract}




\subsection{Introduction}

The use of tools is one of the most remarkable milestones in the history of human kind. Nonhuman primates, such as orangutans, have also been observed creating tools and using them for hunting [45] or having access to food [231], both in natural and restricted ecosystems [292]. Moreover, they are capable of using objects in their environment as tools for more general purposes: from instinctive reactions such as using a stick to hit unknown objects to more logical behaviors such as measuring a rivers' depth using a long branch to touch the bottom. This shared ability of primates, which has evolved differently between humans and non-human ones, is one of a kind example to realize how similar we are and compare our similar abilities and reactions.

The manipulation of tools or objects is frequently used as a form of enrichment for primates in zoo enclosures, usually to stimulate them to get food rewards [356]. Toys and everyday objects of different shapes and materials are also used to foster physical activity and appeal to animals' innate playful behavior [65, 254, 258]. In terms of enrichment, the use of interactive technologies into the zoo has opened a whole new area of possibilities to create activities which improve animal welfare while raising awareness of zoo visitors. However, current enrichment practices based on technology differ significantly from the ones in which no technology is used. One of the main reasons is the dangers and threats to animal welfare that could pose letting spontaneous and powerful animals, such as orangutans, manipulate fragile technological devices. Therefore, one of the most viable options seemed to be the use of touchscreen computers, as similarities between humans and primates led to the belief that their use would be easy for them [10, 29]. However, in this kind of activities the interaction is not as natural for the animal as it is for a human: the device has to be held by a zoo keeper near to the primate's cage, and the primate has to interact with the device through the bars of the enclosure. Moreover, the primate has to understand the metaphors of the graphical interfaces on the touchscreen in order to fully comprehend how the system works, or either just interact with it and observe the reactions of the system [364]. Additionally, the joy ends whenever the zoo keeper leaves: primates cannot use these enriching elements when there is no human present. Furthermore, zoo visitors are not able to participate in these activities, which usually take place out of the visitors' side of the zoo. This prevents visitors from observing with their own eyes how similar we are to our primate companions and have a closer experience with endangered species, which could be an insightful educational activity.

In this paper we propose a new approach to provide technological enrichment for primates based on their innate abilities for manipulating tools and objects. We also address how collaborative activities based on the manipulation of objects could facilitate the interaction between humans and orangutans, raising awareness of wildlife preservation in zoo visitors. 


\subsection{Proposal and Research Agenda}

A promising form of enrichment for primates which exploits their natural ability of using tools/objects could be the development of digital interactive experiences mediated by the use of non-technological tangible objects which primates can actually hold and manipulate [364]. In this kind of systems, primates could use regular objects that they know and are used to interact with. The interactive experience could be provided by analyzing and tracking the gestures and interactions of the primate with the object/s and providing digital responses, such as projections on the ground/wall, as reactions to these manipulations. In this way, natural behaviors and interactions of the orangutans would not be restricted or limited. These natural interactions with the devices could inform the design of the interactive experience, adapting the reaction of the system to better suit the abilities and gestures of the animal with the object, and not the other way around. Moreover, technological elements not only could offer enrichment for primates, but also for primates and humans. The use of objects/tools, which is a common ability between both species, could facilitate interspecies communication when using similar objects in a shared technological space [362], while raising awareness of humans on the commonalities with our primate ancestors.

The first step towards the creation of these systems would be evaluating different tangible elements regarding the natural interaction and interest of the orangutans towards them, selecting the most appropriate ones. Then, the interaction of the selected elements could be enhanced with digital responses. Through tracking systems, we could learn which kind of interactions, gestures and responses the orangutans perform in each game, their reactions and observed behavioral changes. This will inform the redesign of the activity in terms of these natural gestures, in order to make the interaction as natural as possible and adapted to what the orangutan expects from it. Similar interactive spaces could be deployed on the visitors' side, in which visitors have to manipulate similar objects, triggering also digital responses. In this case, the digital responses could have an educational purpose, such as projecting a video of the orangutan using the same object in its environment. The visitor could observe the orangutans' intelligence and physical abilities and also reflect upon those behaviors by comparing orangutans with human beings. The manipulation of tangible objects by a human visitor could also trigger digital responses on the orangutans' side to capture their attention. In this way, a shared space could be created based on non-verbal communication and/or mimicking based on the manipulation of the same objects and the digital interactions of both human and orangutan. Collaborative tasks could then be created, in which the orangutan and the human have to cooperate and use tools/objects to achieve the goals of the activity, fostering communication between species mediated by the objects they are manipulating. 
Table 7.1: Examples of tangible-mediated activities.

\begin{tabular}{|c|c|c|}
\hline Tangible tools & Individual activities & $\begin{array}{l}\text { Collaborative activities } \\
\text { between animal/human }\end{array}$ \\
\hline $\begin{array}{l}\text { - Plastic balls } \\
\text { - Plush toys } \\
\text { - Sticks } \\
\text { - Treat tubes } \\
\text { - Books } \\
\text { - Wooden } \\
\text { bricks }\end{array}$ & $\begin{array}{l}\text { - Painting-like application } \\
\text { using a tangible element } \\
\text { and projections on the } \\
\text { ground/wall } \\
\text { - Whack-a-mole game using } \\
\text { a tangible object and pro- } \\
\text { jections on the ground } \\
\text { - Produce sound as a re- } \\
\text { sponse to the gestures per- } \\
\text { formed with a tangible } \\
\text { object, different gestures } \\
\text { could play different sounds }\end{array}$ & $\begin{array}{l}\text { - Pong: tangible objects } \\
\text { which both human and } \\
\text { orangutan use to move a } \\
\text { digital ball projection on } \\
\text { the floor } \\
\text { - Puzzle: human and } \\
\text { orangutan use blocks } \\
\text { to complete a puzzle } \\
\text { projection on the floor } \\
\text { - Music creation: object ma- } \\
\text { nipulations by both the } \\
\text { orangutan and the human } \\
\text { would produce sounds }\end{array}$ \\
\hline
\end{tabular}

\subsection{Benefits}

The benefits of this kind of activities are manifold. On the orangutan side, new ways of mental and physical stimulation will be offered by means of enriching interactive spaces. By introducing non-technological objects which enact technological reactions on the system, a new road opens towards providing enrichment which does not require human activation. The innate curiosity towards the system could also be evaluated and used to inform further designs of zoo enrichment. On the human side, it would be an educational activity for zoo visitors', both adults and children, who will realize the importance of the use of tools in the evolution of primates, with obvious evidences on how close our species are in terms of abilities and intelligence. Visitors experience will be enriched as they could engage into a more direct interactive experience with the orangutans. This could create affective bonds towards the species and facilitate further implication of visitors into conservation activities.

\subsection{Conclusions}

This paper proposes the development of digital interactive activities for primates based on the use of non-technological tangible elements. The manipulation of these objects would trigger digital responses of the system, such as digital projections. With these scenarios, we aim at improving the wellbeing of captive animals by providing enrichment activities which happen inside the animals' enclosure and with 
objects they can actually manipulate and examine. The responses of the system will be based on the natural manipulations of the objects after careful exploration of the most suitable elements and interactions for primates. Collaborative interactive spaces between primates and zoo visitors could also be developed, in which tangible objects are used to mediate the interaction. Zoo visitors' experience would in this case be enhanced as they could interact with the primates during these activities, potentially increasing their comprehension of the species and the importance of nature conservation.

\section{Acknowledgements}

This work is funded by the European Development Regional Fund (EDRF-FEDER) and supported by Spanish MINECO with Project TIN2014-60077-R. The work of Patricia Pons is supported by a national grant (FPU13/03831) from the Spanish MECD and an Endeavour Research Fellowship from the Australian Government. 



\title{
Chapter 8
}

\section{Sound to your Objects: A Novel Design Approach to Evaluate Orangutans' Interest in Sound-based Stimuli}

\author{
Patricia Pons ${ }^{1}$, Marcus Carter ${ }^{2}$, Javier Jaen ${ }^{1}$ \\ ${ }^{1}$ ISSI Group, Department of Computer Systems and Computation (DSIC), \\ Universitat Politècnica de València, Valencia, Spain \\ ${ }^{2}$ Microsoft Research Centre for Social Natural User Interfaces, \\ University of Melbourne, Australia
}

Published in Proceedings of The Third International Congress on Animal Computer Interaction, pp. 7:1-7:5, ACM (2016). doi:10.1145/2995257.2995383

\begin{abstract}
Orangutans show interest in sound-based stimuli, but the auditory enrichment they are usually provided with is either based on human-music, or does not allow for control and choice. In this work-in-progress paper, we describe the design of soundbased enrichment by means of the manipulation of tangible non-technological elements. In doing so, we demonstrate how Animal Computer Interaction research can help in providing animals with more control over auditory stimuli, as well as demonstrating and justifying a novel modality for interaction based on orangutan
\end{abstract}


behavior within our ongoing study. We overview our proposed evaluation, identifying how - through embodying notions of control and choice in design - our proposed system allows for orangutan use to inform ongoing development and design of auditory enrichment.

\subsection{Introduction}

Animal enrichment is essential for providing captive animals with engaging and adequate stimuli to "draw out their species-appropriate behaviors, thus enhancing animal welfare" [64]. Zoos, in their role as conservational organizations, make huge efforts towards providing captive animals with naturalistic as well as stimulating environments [41]. Recently, the field of Animal Computer Interaction (ACI) $[173,174]$ and zoo practices for animal enrichment have joined efforts towards the design and development of technologically mediated enrichment activities [341]. Technology can offer a whole new range of opportunities for animal enrichment, allowing for more rapid adaptation to new circumstances, specific individuals, and easily introducing new activities within already tested systems, e.g. new tabletbased applications for primates [10]. This rapid incorporation of new activities offers the animals more opportunities for exploration and could help to maintain their engagement and interest.

Auditory stimuli are a widespread form of environmental enrichment, particularly for primates. However due to safety or environmental restrictions, primates have little control over the provided stimuli and interaction has been restricted or non-existent. As a result, enrichment is unable to account for species or individual preferences. Several studies have tried to determine the best music stimuli for different primate species, re-appropriating human-music for the purpose. These studies either imposed an unsuitable physical set-up [188], did not allow for choice or creation, or were focused just on musical pieces [275] and not sound stimuli over which the animal has more control and choice.

ACI research can help overcome these limitations and provide primates with more control, intuitive interactions and adaptations of these auditory stimuli. In this work-in-progress paper we present the design of a digital sound-based auditory enrichment, based on a novel interface which interprets the movement of nontechnological objects as digital input, overcoming safety and technology access concerns. Instead of providing primates with what we believe it is music to their ears, our novel interactive approach gives sound to their objects. In this approach, orangutans can explore producing different types and variations of sound-based stimuli by manipulating tangible non-technological objects [252]. By providing control and choice over sound generation, the individual preferences of the animals towards the sound-based stimuli can be studied. 
This paper consequently contributes; (1) an account of our animal-centered design process for developing novel digital enrichment for orangutans in a zoocontext, (2) a novel and promising approach for mediating animals' interactions with technology, and (3) a sound-based interactive system designed for orangutans which embodies notions of control and choice in its design as a resource for cocreation. We conclude by overviewing our proposed evaluation.

\subsection{Related Works}

There have been many studies analyzing the behavioral and physiological effects of auditory-stimuli for primates in captive facilities, which provided contradictory or non-conclusive results. Some studies reported decreased stereotypical behaviors when primates were listening to music [125, 335], others found no difference in behaviors but instead on physiological measures [32], and some works found differences in both [169]. Several studies have also tried to analyze primates' preferences towards different genres of music, also without yielding consonant results. McDermott et. al. [188] defined a physical set-up which conducted to a least-aversive selection [276]. On the contrary, the work by Ritvo [275] provided orangutans the opportunity to conduct a most-desired choice over different genres of music.

However, none of these works allowed the animals to control which kind of music they were listening to. Moreover, the considered auditory stimuli were human-based musical pieces, reflecting the imposition of human-centered enrichment. Instead of music, sound-based stimuli could be more appropriate when the appropriate levels of control and choice are afforded to the animal user. In an ACI user-centered perspective, orangutans as target users should be the ones dictating what music is for them. In fact, a recent attempt to create species-appropriate music for cats using non-human centric sounds has shown promising results in terms of cats' interest in the auditory stimuli [305].

Within ACI, the use of sound-based stimuli with orangutans has been observed to foster interaction and exploratory behaviors. Hermans and Eggen have developed a cylinder which produces automatically generated instrumental sounds when rotated [111]. Although orangutans showed interest, they had no control over the generated sounds. One step further, Wirman has proposed the development of a game prototype for orangutans in which various poking sticks are used to explore sounds [364]. In this promising work, the interaction has been designed based on the observed preferences and interactions of the orangutans. This approach would account for a more intuitive interaction, allowing the orangutan to focus more on playful interactions and explorations of the system. The work presented in this paper is aligned with this latter idea of exploration and describes the design of a system that would allow to study how orangutans want to interact with auditory 
stimuli of different kinds, and which type of sounds they would prefer to interact with.

\subsection{Design Process}

Here, we present the design process for the development of a sound-based interactive system for orangutans, following an animal-centered approach [173]. The reactions and demonstrated preferences of the orangutans when using the system will inform the design [142]. Zoo keepers also participate in the process with their expertise and knowledge of the orangutan users. We divide the decision making of our design process into three main areas: (1) the selection of tangible elements as mediators of the interaction, (2) the technical challenges to support this kind of interaction, and (3) the selection of suitable and potentially appealing sound-based stimuli.

\subsubsection{Tangible Interactions}

Environmental enrichment devices (EEDs) are used across zoos as one of the main forms of enrichment for animals. The use of tools and objects for enrichment purposes is usually meant to simulate the animals' eating and foraging habits in the wild. Toys and everyday objects of different shapes and materials are also used for providing amusement activities for the animals as well as mental stimulation. Physical activity as well as social relationships are also fostered through the use of objects in their environment. Moreover, there are several animal species, including orangutans, with innate abilities and preferences towards the use and even creation of their own tools [292].

Technology is extremely limited in supporting this mode of enrichment, particularly with primates who are extremely strong, potentially harming themselves on deconstructed technology. Hence, orangutans do not usually have free access to any technology inside their enclosure, instead being handled by a zoo keeper or professional staff. This limits the time in which the animals have access to the technology and impedes their control and choice over when to access the enrichment, and how to interact with it.

The Kinecting with Orangutans project [343] provided digital enrichment to the orangutans at Melbourne Zoo by utilizing a Microsoft Kinect device to create touch-aware projections. In doing so, this project allowed the orangutans to interact with the digital projections how they wanted to, and they were given regular free access to the technology over the course of a month. This saw the orangutans interact with the technology in numerous unexpected ways, such as with their body, and also with objects (see Figure 8.1). This included: spreading wood-wool (nesting material) over the projection, interacting with the projection with toys 
(plastic balls), which also afforded other means to interact (such as hanging from the ceiling and 'whipping' the screen with a blanket to interact, or attaching a tarpaulin from which the orangutan could hang to interact). These interactions were unanticipated, but afforded by the touch-detection software.

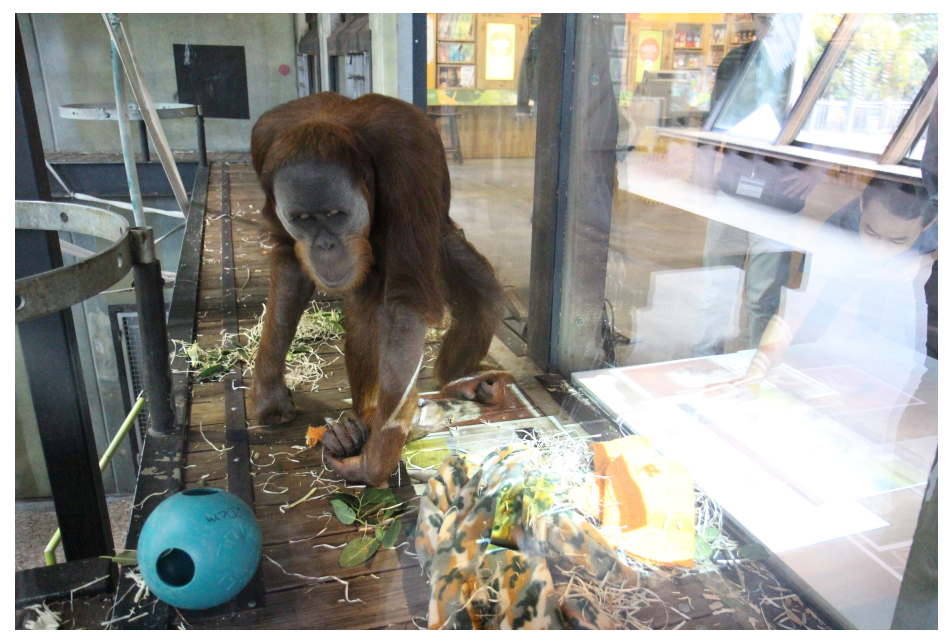

Figure 8.1: Orangutan using objects to interact with projection

Based on this predisposition and intrinsic ability of orangutans towards the use of objects, we concluded that a promising and novel approach for interacting with technology would be the design of a system in which these non-technological objects could afford digital interaction remotely, thus overcoming the safety and physical constraints of technology with primates [252]. Such a system should allow animals to manipulate and interact with everyday non-technological objects inside their enclosure and obtain adequate digital reactions from the system based on these interactions. These responses should be triggered by the system when the manipulations happen from any location and posture within the interaction area. The orangutans' spontaneous manipulations of the object will not be limited or restricted by specific interaction constraints. Moreover, the interaction should not be limited to a specific object. Our proposed approach thus allows orangutans to interact with the system using different elements they have around without requiring to put any special artefact on the object. This novel approach for interacting with digital systems would provide the animals with more control over their environmental enrichment. Eventually they will be able to choose whether and when they want to interact and in which ways by simply manipulating (or not) the objects mediating the interaction. 


\subsubsection{Technical Development}

In order to augment the interactions with non-technological tangible objects, the main challenge comprised how to remotely detect the movements of these objects. An approach such as [257], using depth based information to detect postures was not considered to be as effective with orangutans. A top-down approach from a tracking sensor will not allow for the detection of the hands movements. Moreover, orangutans are likely to manipulate objects from many different postures and not always standing or sitting but also hanging or lying on the ground. Therefore, it was decided that an approach in which the system tracks the objects would be more effective and flexible.

After observing and discussing with Zoo Keepers which kind of everyday objects could be used to mediate the interaction, a wide range of colors, shapes and textures were identified. We considered several approaches for object recognition [366], such as pattern matching. However, this approach would not account for objects made of a non-rigid material. It would also make more complex the recognition of the same object in different positions and rotation angles without previous analysis of the object. The two main challenges to overcome were defined as: allowing for flexibility in the range of objects to be used as mediators, and allowing for easy and rapid incorporation of new objects into the system. We concluded that performing object tracking based on color would be most suitable as objects of different shapes and textures could be used. This technique also allows tracking the object even when it is being partially occluded or handled by the orangutan. Moreover, adding new objects to the system just requires to define the main distinctive color of the object. The only restriction would be that this color is different enough from the surroundings.

The developed system consists of a Microsoft Kinect ${ }^{\circledR}$ v2. This sensor captures both color and depth information of the recorded area at a rate of 30 frames per second, with pixel resolutions of 1920x1080 and 512x424 respectively. The software has been developed using C\#, Kinect for Windows SDK $2.0^{1}$ and EmguCV ${ }^{2}$ (a .NET wrapper for OpenCV). The Kinect sensor acquires both color and depth streams of information. For each pair of color and depth frames, the system converts the color stream to the Hue-Saturation-Value (HSV) color space. Then it filters the HSV image using the HSV values of the object being tracked. Over this image, which only contains the blobs of the object/s being tracked, it applies a blob extraction algorithm to obtain the blobs representing the objects. With this information, the Kinect SDK is used to determine the position and depth of the tracked object within the image. At this stage, different modalities for producing sounds can be triggered attending to the position and movements performed with the object/s.

\footnotetext{
${ }^{1}$ https://msdn.microsoft.com/en-us/library/dn799271.aspx

${ }^{2}$ http://www.emgu.com/
} 


\subsubsection{Sound-based Enrichment}

Auditory stimuli are frequently used with Melbourne Zoo's orangutans. This includes using electronic keyboards, harmonicas, iPad music applications, playing sound from CDs or radio, and when accompanying projected movies. Keepers and researchers widely report on primates' curiosity towards sound-based stimuli $[111,364]$. In consideration of our interest in providing control and choice to the orangutans, we concluded that the auditory stimuli should be open to exploration, surprise, and engagement, based on how Zoo Keepers identified the opportunity for such technology and prior work $[142,252,364]$. We considered stimuli from simple notes or sounds to more complex compositions, without the condition that it needs to sound "nicely" or be "harmonious". Nature sounds were discussed, but have been observed to increase vigilance behaviors in captive gorillas [229] due to the fact that they could not locate the origin of the sound, and therefore were not considered. Instead, the fact that the sound was familiar or recognizable to the orangutans (all raised in captivity) was more important.

Several basic modes of producing sounds based on the manipulations or movements of an object were initially implemented. These basic modalities were shown to the Zoo Keepers to provide them with an idea of the capabilities the proposed system could have, prompting discussion and brainstorming ideas of potentially appealing auditory stimuli The Zoo Keepers' knowledge about the individual personalities of orangutans and the way they interact or produce auditory stimuli during everyday enrichment activities were an extremely valuable source of information to inform the decisions of the sound modalities to be implemented and the kind of sounds and mappings these modalities should offer. After discussion, the following three different modalities for generating sound have been developed for the evaluation of a proof of concept system:

- Noise: Noisy and clanking sounds are generated each time the object is moved. Design decision: Orangutans have been observed producing noise with objects around them.

- Progression: A melody is played while the object keeps moving. If the object is not being moved, the melody is stopped. When the object is moved again, the melody restarts playing from the last point it was stopped. Although this would be closer to music than to sounds, in this case the melody does not need to sound good (it could be stopped and replayed at the orangutans' will). Volume variations of the sounds are also produced based on the speed of the movements performed, i.e., if the object is moved fast the volume would be increased, and if the movements of the object decrease in intensity then the volume would be decreased as well. Design decision: Playing music from CDs, radio or while projecting movies is a frequent form of auditory enrichment at the Zoo and orangutans could recognize the melodies in this modality. 
- Continuous: Different notes are generated continuously when the object is within the tracked area. The notes change depending on the position of the object and distance to the sensor. When the object is placed far away from the sensor the notes produced are low-pitched, and vice versa. Volume variations are also produced based on the speed of the movements performed. Design decision: Orangutans are curious about new things happening in their environment. Sound variations within this modality would foster exploration and sustain the novelty factor.

Several sounds have been incorporated into each modality. It should be noted that even though terms such as pitch and note are used here, and this might be terms used by human beings to describe auditory stimuli, it is just a representation for us to understand which changes in the sound stimuli are presented. We ground on the knowledge that orangutans can distinguish between different sources and variations of sounds $[188,205]$, hence notes with different pitch or melodies with different rhythm should be perceived as different stimuli. These variations in the source of the stimuli aim to provide variety in order to foster curiosity, engagement and exploratory behaviors. As the movements of the tracked objects will trigger different sounds, this would allow to study how orangutans prefer to interact and manipulate the objects to, for example, replay a specific sound or keep moving the object to discover new ones. Hence, we could also study which auditory stimuli are more engaging based on the performed movements and analysis of their behavior.

\subsection{Conclusion and Future Work}

This work has presented a novel interactive approach for mediating interactions within ACI. This approach is based on the use of non-technological tangible objects as proxies to interact with the system. It could be broadly applied within ACI to provide not only enrichment but also control over the environment for different animal species. The developed solution is capable of tracking objects of different shapes, colors and textures. This tangible approach has been applied to the context of designing a sound-based interactive system for orangutans with the aim of providing a more intuitive form of interaction for animals as well as more control over their environmental auditory enrichment. An evaluation of the sound-based interactive system will be conducted in order to study: (1) the application and suitability of the proposed tangible interactive approach, (2) orangutans' interest in sound-based stimuli in terms of their interactions and explorations with the system. The results of this study would help to discover similarities and differences between individuals when interacting with the sound-based stimuli in terms of behavior, movement patterns and activity levels. The proposed interactive approach based on tangible elements would offer the orangutans more control and choice over the provided enrichment, hence these results would inform the design of similar and more permanent installations for animal enrichment based on 
their preferences on auditory stimuli. It will also help to envision how intelligent adaptations of the system could fulfill species and individual specific needs and preferences [254, 258].

The tangible interaction approach could be used not only for orangutans but also for other species of animals with the ability of object manipulation, or in which the use of objects is among their preferred interactions with the environment, e.g., other species of primates, elephants, big cats, etc. Moreover, tangible interactions could not only be used with sound-based stimuli. Visual stimuli such as projections on the ground or walls could also be triggered by the system, these projections reacting according to the interactions of the animals with the tangible items. In addition, this form of interaction could be used to provide animals with control over their environment. Instead of using metaphors as buttons or activators, the use, movement or placement of objects could be mapped to changes over environmental features of the enclosure, such as lighting conditions or even playing sounds or movies if it is found that the animal would like to have this option. Allowing animals to have control over their surroundings in this kind of enclosures is one of the keys to improving their welfare.

Collaborative activities with zoo visitors or remote interactions with humans not present in the enclosure could also be envisioned using tangible metaphors to interact with the system. The use of tools as mediators of the interaction could be conceived as a way of connecting the human participant with the orangutan by relying on the common ability of both species regarding object manipulations.

\section{Acknowledgements}

This work is funded by the EDRF-FEDER, Spanish MINECO (TIN2014-60077-R), Spanish MECD (FPU13/03831) and Australian Government (Endeavour Research Fellowship). The authors gratefully acknowledge the invaluable support of the Microsoft Research Centre for Social NUI and Zoos Victoria. 



\section{Part IV}

\section{Interspecies Participatory \\ Design of Playful Interactive Environments}



Human-animal interactions have been shown to be beneficial for both parts of the interplay $[12,13,215]$, and technologically mediated experiences offer a whole new range of opportunities in this regard, especially if we consider how important it is that future generations raise their awareness and empathy towards the animal species living in our world.

Once the animal side of the interaction has been explored, this part of the thesis focuses on how to support children's participation in interactive spaces for animals by means of NUIs. For this purpose, several evaluations were conducted to allow the children's to co-design and participate in interactive and intelligent playful scenarios for animals, and to assess the impact of these activities on improving the children's perceptions of animals and the children's perceived wellbeing. The insights gained through these studies helped to complete the development of an interspecies interactive playful system that allows for remote interaction between children and animals, as well as animals playing by themselves, that will be presented at the end of this part of the thesis.

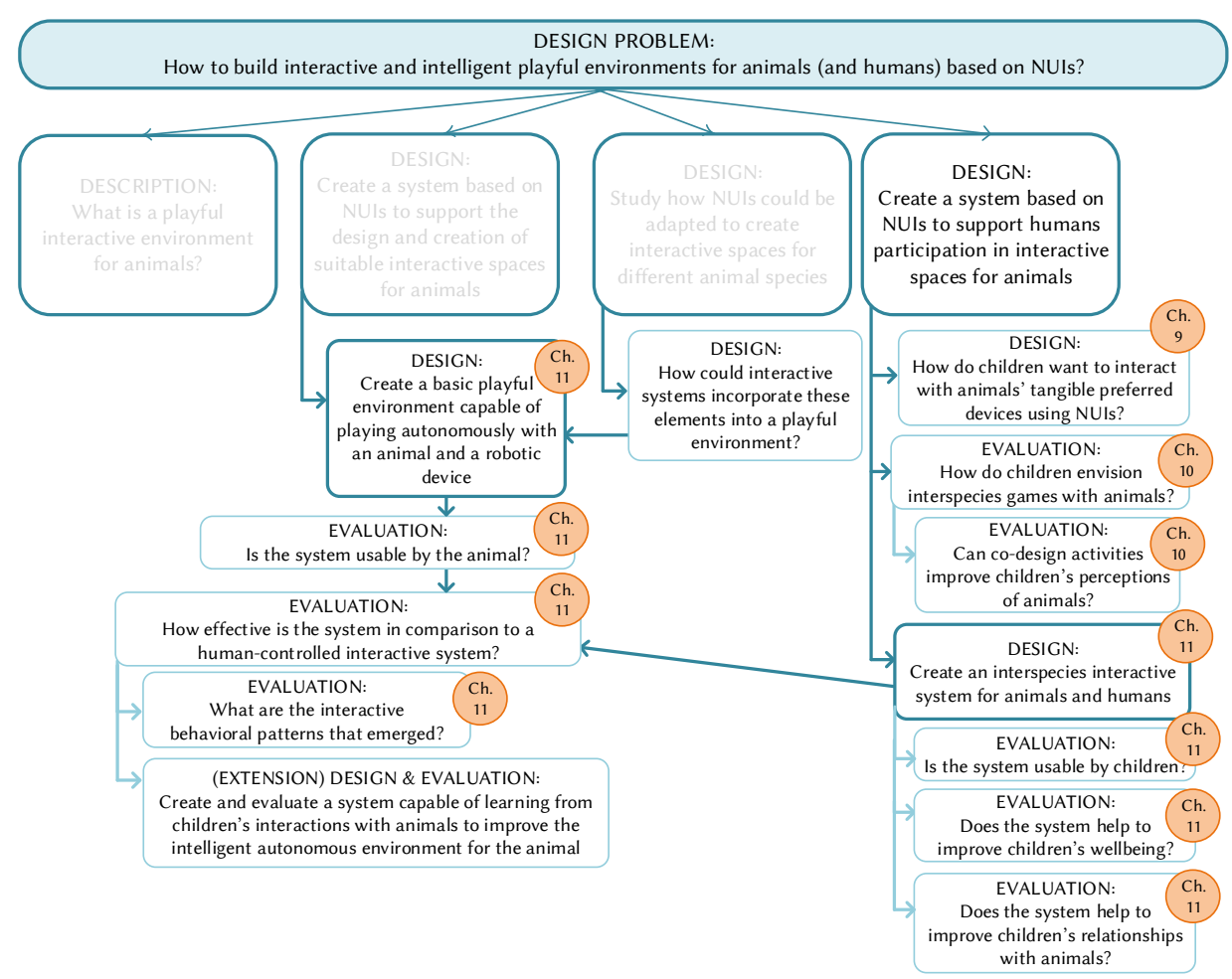

Figure 8.2: Problem decomposition of the work described in Part IV. 



\title{
Chapter 9
}

\section{Interactive spaces for children: gesture elicitation for controlling ground mini-robots}

\author{
Patricia Pons, Javier Jaen
}

ISSI Group, Department of Computer Systems and Computation (DSIC),

Universitat Politècnica de València, Valencia, Spain

Submitted to Journal of Ambient Intelligence and Humanized Computing. Under review, as of June 2018.

\begin{abstract}
Interactive spaces for education are emerging as a mechanism for fostering children's natural ways of learning by means of play and exploration in physical spaces. The advanced interactive modalities and devices for such environments need to be both motivating and intuitive for children. Among the wide variety of interactive mechanisms, robots have been a popular research topic in the context of educational tools due to their attractiveness for children. However, few studies have focused on how children would naturally interact and explore interactive environments with robots. While there is abundant research on full-body interaction and intuitive manipulation of robots by adults, no similar research has been done with children. This paper therefore describes a gesture elicitation study that identified the preferred gestures and body language communication used by children to
\end{abstract}


control ground robots. The results of the elicitation study were used to define a gestural language that covers the different preferences of the gestures by age group and gender, with a good acceptance rate in the 6-12 age range. The study also revealed interactive spaces with robots using body gestures as motivating and promising scenarios for collaborative or remote learning activities.

\subsection{Introduction}

Technology has been shown to be a promising and motivational tool for educational purposes with children. In this regard, over the last years there has been a shift from desktop-based interactions between children and digital services for learning, towards more ubiquitous and intuitive scenarios. This has favored the emergence of interactive spaces for education aimed at fostering children's natural ways of learning by means of play, exploration in physical spaces and tangible manipulation $[3,100,184,200]$. For these reasons, these scenarios are not just purely virtual any more but instead are interactive spaces including advanced interactive modalities, such as augmented interactions, and non-digital artifacts, such as tangible devices of different kinds [28, 86, 306]. Among the wide variety of tangible interactive devices that are available in these spaces, children find robots attractive and intuitive. In fact, robots have been a popular research topic in the context of educational tools. Interactive activities between robots and children have the potential to foster physical activity, trigger creativity and provide a more engaging learning experience [86, 288, 317, 338].

In order to provide users with more freedom and spontaneity in their interactions, advances in Natural User Interfaces (NUIs) have been applied in interactive spaces for adults [23, 69, 140, 331, 358]. Research on NUIs has widely covered how adult participants would like to interact with such novel interactive spaces. There is now a vast literature on gesture elicitation studies for adults in different contexts [155, 202, 203, 245, 332], which also demonstrates that user-defined gestures are usually better accepted by end-users [55, 203, 263]. On the contrary, less research has focused on how children would prefer to interact in these scenarios. In the particular case of robots, research has also studied how adults would naturally interact using their full body for the navigation and control of these physical elements [43, 195, 226, 227]. However, few platforms for children offer gestural and body language communication with these interactive elements. Gesture-based communication with robots would be especially relevant within interactive spaces, as for example, voice commands might not be accurate if there is ambient noise or there are many children playing at the same time. In addition, current scenarios in which children can interact using body language are usually based on virtual environments, and the gestural languages have already been predefined by the developers. Therefore, studying how NUIs and body language communication could 
provide children with a more intuitive and motivational experience for controlling tangible robots within interactive scenarios would be a promising research line.

To the best of our knowledge, this paper presents the first gesture elicitation study with children regarding full body interactions to manipulate tangible ground robots. The outcomes of this elicitation study are manifold: first, the study identifies the preferred gestures and body language communication that children would like to use in interactive spaces with robots. It also outlines the variability of such gestures by age group and gender, proposing a gestural language that accommodates for the detected requirements. Secondly, the positive results from the questionnaires reveal that interactive scenarios with robots using body gestures are very promising and motivating for children. Finally, a set of future recommendations based on the elicitation study and the postquestionnaire results is outlined. These discussion aims to advance research in the design and development of promising interactive spaces for children based on natural interactions.

\section{$9.2 \quad$ Related Works}

This section firstly reviews related studies on children's communicative forms of interaction within interactive spaces and with robotic systems. Then, studies focused on gesture and full-body elicitation techniques with children are described, highlighting the need for more participatory design processes with children in these areas. Due to the limited number of gesture elicitation studies with children regarding interactive spaces and tangible robots, we propose revisiting the related work in this area focused on adult participants.

\subsubsection{Children's Communication with Robots and Interactive Spaces}

Child Computer Interaction (CCI) [124, 268, 269] research has expanded greatly over the last decades, partly due to the realization that children's mental models are different from those of adults. Hence, interactive technologies for children that aim to be successful need to adapt to these mental models and preferred ways of interaction. In this process of understanding and adapting technology to better suit children's requirements, cooperative inquiry and participatory design with children have been among the main pillars of the field [57, 59]. These methods have been used in search of motivating and playful technology that encourages exploration and learning in children, and interactive spaces and robots have emerged as very promising tools for this purpose $[9,123]$.

On one hand, interactive spaces are moving towards full-body interactions in which children can behave naturally using their whole body to interact with the system. This offers a more natural interactive modality while encouraging exercise 
and socialization. Back in 2000, the KidsRoom [27] and StoryRooms [3] proposed interactive storytelling experiences for children that took place in an interactive real world room. Over the years, advances in technology have made these immersive scenarios more affordable and sophisticated. For example, interactive floors such as iGameFloor [100] or Lands of Fog [200] allowed full-body interactions for collaborative learning and socialization using computer vision techniques. Other interactive playgrounds for children that considered full body interactions have been the interactive slide [306], gesture-based digital games [95, 128, 236] and hybrid digital and tangible games [28].

On the other hand, interactions between robots and children have been studied from different perspectives and offering several interactive modalities. However, few works have addressed embodied interaction techniques between children and robots. Those works in which embodied metaphors are present are usually focused on imitation and social interactions for therapy, e.g. socialization with robots for children with autism [277, 278, 338]. Embodied interactions and body posture have also been analyzed to detect children's engagement and social interactions with robots [110, 194, 288, 289]. However, all these studies consisted of interactive modalities already pre-programmed by the designers, and the children had to adopt them in order to interact with the robot. Some examples include verbal communication [22], gesture imitation [277, 338], touch-based interactions [194, $278,288]$ or tangible-mediated interactions [86, 209].

The proposed interactive modalities have been quite varied as regards children's control and manipulation of a robot. Topobo robot [265] required children to perform physical programming by example, i.e. assembling the robot and recording the desired movement by moving its physical parts, then playing the recorded movements. LEGO sheets proposed a visual programming language for the LEGO Programmable Brick, a physically assembled LEGO robot [92]. This allowed the robot to be programmed with predefined sequences of actions, but did not provide real time control of it by the children. A similar proposal was Robo-blocks [304], which offered a tangible approach for programming a floor robot by assembling physical command blocks. However, it offered only four basic actions: forward, backwards, left and right. The Tangibot platform [86, 209] proposed a more collaborative real-time technological solution. It consisted of a tangible-mediated user interface for kindergarten children to manipulate a mobile robot. The work in $[315,317]$ also proposed a tangible interface for children in the form of a tricycle to remotely control a robot. The movements performed on the tricycle were mapped to movements of the tele-operated robot. However, it did have some technical limitations, as the child operating the robot could not perform fine-grained interactions and could not move further when he reached a wall, etc.

There is thus a shortage of studies on how full body interactions that work well in interactive spaces for children could be applied to manipulating robots in such spaces. We believe the study of robot control by gestural communication 
in an interactive space will offer promising opportunities to develop motivating and engaging technological scenarios. The following sections will review elicitation studies on both children and adults to give a general idea of the range of methodologies that could be applied for the purpose of this study.

\subsubsection{Elicitation Studies with Children}

Gesture elicitation studies with children have been used as a tool to include children in the design process of a system $[58,59,101]$ following a participatory design approach [53]. However, the relatively few studies in the area mostly focus on more traditional user input interfaces, such as tabletops [287] or digital videogames [127].

Regarding interactive surfaces, a methodology proposed by Wobbrock et al. [365] within adult elicitation studies has been adapted to study how children designed new gesture-based interactions on tabletops [287]. In this study, after observing an animation representing some referent action, the children were asked to come up with a gesture to trigger that action.

Höysniemi et al. [126, 127] conducted an elicitation study in which children had to perform full body interactions to control a digital character in a virtual game. This work used a Wizard of $\mathrm{Oz}$ elicitation technique, showing this is a feasible methodology well accepted by children, despite the delay between the child's action and the reaction of the character.

With the emergence of depth-based tracking technology such as the Microsoft Kinect sensor, gesture elicitation studies with children have also evaluated how they would like to manipulate digital objects which appear on screen [49]. Such systems allow for rapid prototyping and usually facilitate the gesture recognition task with built-in frameworks. However, they still have limitations in terms of open space tracking and gesture precision, which is a drawback for less restricted scenarios.

In the context of interactive environments for learning, bodystorming techniques have been used to elicit children's full body interactions to improve their communication with the system $[172,293]$. In these studies, children were asked to use their bodies to represent possible actions and relationships between elements in order to address issues related to air-pollution. Motivation was reported as high among the participants, although the proposed gestures were more focused on representing the different actors of the scenario rather than solving the design challenge. Although the authors acknowledge some of the representations cannot be directly mapped to the final system, full body interactions were a powerful tool within the participatory design process with children [294]. 


\subsubsection{Elicitation Studies with Adults}

In contrast to the reduced number of works regarding gesture usability studies with children, research focusing on gesture elicitation studies with adults has been extensive.

The widespread use of handheld devices motivated a great number of studies for eliciting natural gestures for mobile phones or tablets. Some of the works in the area include elicitation studies to use the back of the mobile device as an input [300], or use motion gestures to trigger actions on the device [284]. Considering more collaborative and interactive scenarios, several studies have addressed how users would like to interact with different devices in a multi-display environment in order to transfer files or share content $[155,157,298]$. The methodologies of these elicitation studies were in the form of interviews or guided tasks: the participant was asked to perform a gesture after reading a description of the task or observing a short image/video of the results for the proposed action.

Hand gestures for vertical displays (TVs or interactive walls) have also been elicited using different approaches. For example, online surveys in which participants propose gestures for a specific command using open-ended answers [55]. Another common procedure consists of the participant performing a gesture after observing a short video demonstration of the proposed command [332]. And finally, Wizard of $\mathrm{Oz}$ methodologies, in which the referent action is initially shown to the user and also is triggered while he performs the action [163, 202]. With the emergence of tabletops, gesture elicitation studies were also conducted to elicit user-defined gestures for interactive surfaces [66, 365]. Methodologies reported in the literature using these platforms consisted of showing an animation of a referent action to the participant, and asking him to perform a gesture that would prompt that action.

Moving to more immersive interactive spaces, elicitation studies for collocated interactions with wearables have also been conducted [138]. Regarding digital games, full-body gesture elicitation procedures [151] required the participant to play a digital game standing in front of a screen. Whenever the game script stops and displays different options for the character to continue the story, the participant is asked to perform a gesture that represents each option. User defined gestures have also been elicited in Augmented Reality (AR) scenarios. Using a head-mounted device, participants are shown an animation of the desired result and then asked to perform a suitable gesture to cause that effect [245].

Several elicitation studies focused on adult manipulation of either humanoid robots $[225,227]$ as well as flying drones [43, 226]. The methodologies adopted in these studies usually follow a Wizard of $\mathrm{Oz}$ approach: (1) the user is shown a video of the robot/drone performing a referent action or reads its description on a card, (2) the user performs a gesture that represents the referent action, and (3) the 
robot/drone performs the desired action. In addition, there is an overwhelming amount of studies evaluating drone/robot manipulation techniques for adults, e.g. hand gestures [154, 211, 291], full-body interactions [241, 290], wearables [313], gaze [106], speech [70,311], etc. This contrasts with the few works on the topic with children as participants. For these reasons, and considering how well Wizard of $\mathrm{Oz}$ elicitation techniques work for the user in several contexts, we propose a Wizard of $\mathrm{Oz}$ elicitation study to evaluate how children would like to manipulate a robot using full-body interactions.

\subsection{Elicitation Study}

The main goal of this study is to identify user-defined gestures to control and move a non-humanoid ground mini-robot. This section will describe the study, procedure and the results obtained. The research questions of this work may be formulated as follows:

- RQ1: Is the performance of gestural communicative interaction with robots affected by gender?

- RQ2: Is the performance of gestural communicative interaction with robots affected by group age?

- RQ3: Are there any differences in the kind of gestures elicited by gender and age group?

- RQ4: Is there any gestural language that provides agreement across gender and age?

- RQ5: Is there any difference in the perceived enjoyment of the robot by gender and age group?

- RQ6: Is there any difference in the perceived easiness of the elicitation task by gender and age group?

- RQ7: Is there any difference in the motivation to play by gender and age group?

- RQ8: Is gestural control of robots a promising tool for remote playful activities?

- RQ9: Is gestural control of robots a promising tool for collaborative playful activities?

Section 9.4 will review these research questions reflecting on the results of the proposed study, with the aim of providing a useful discussion that helps future 
researchers in the field to design and develop suitable interactive spaces with robots for children.

\subsubsection{Participants}

The participants were 61 children from a public primary school (Col-legi Públic Vicente Gaos). The participants were classified into three different age groups: early, medium and advanced stage of primary education. The first group (G1) were 21 children (14 boys and 7 girls) aged 6-7 years old (Mean $(\mathrm{M})=6.24$, Standard Deviation $(\mathrm{SD})=0.436)$. The second group $(\mathrm{G} 2)$ were 20 children (10 boys and 10 girls) aged 9-10 years old (Mean $(\mathrm{M})=9.25$, Standard Deviation $(\mathrm{SD})=0.444)$. The third group $(\mathrm{G} 3)$ were 20 children $(10$ boys and 10 girls $)$ aged 11-12 years old (Mean $(\mathrm{M})=11.45$, Standard Deviation $(\mathrm{SD})=0.605)$. The sessions were conducted during school teaching hours.

Before starting the elicitation study, the children were asked whether they had previously used the Microsoft Kinect sensor when playing videogames. Most of them did not know the device before the session, however several children reported having played games with this or a similar sensor: 5 children in G1, 2 children in G2 and 10 children in G3. The children were also asked whether they had previous experience with robots or drones of any kind. In this regard, 4 children in G1, 3 children in G2 and 5 children in G3 reported either owning or having played with a robot/drone before. However, the interaction modalities with such devices had been based on controlling the device with a smartphone/tablet, but never with NUIs such as body tracking.

\subsubsection{Set-up}

The study was conducted following a Wizard-of-Oz approach. A Microsoft Kinect v2 sensor was placed on a table, facing a play area of $200 \mathrm{~cm}$ wide $\mathrm{x} 300 \mathrm{~cm}$ long in which the activities took place. The device video-recorded the activity of the participants for subsequent analysis. The robot used in the study was a Parrot Jumping Sumo mini-drone, placed initially in front of the Kinect. The children were required to stand behind the robot facing the Kinect sensor to start the session, but they could move freely within the play area during the rest of the activity. The researcher stood behind the play area manipulating a smartphone to control the robot (Figure 9.1). 


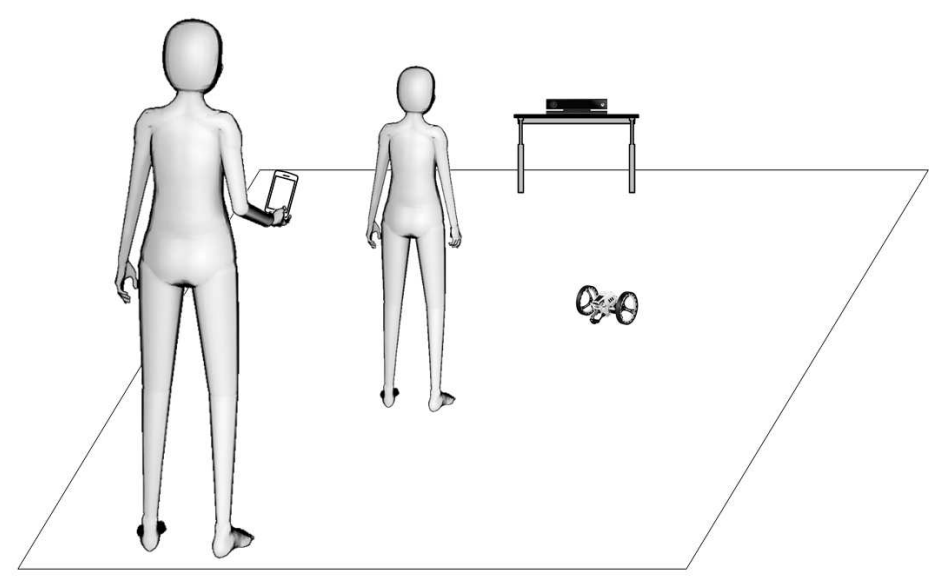

Figure 9.1: Sketch of the Wizard-of-Oz set-up.

\subsubsection{Procedure}

Each child was asked to perform three tasks, with no previous training for any of them, in order to elicit the gestures that children would naturally want to perform to control the robot in each scenario. Task 1 consisted of eliciting gestures for specific and discrete movements of the robot. For this purpose, a set of 11 common actions for controlling mini-robots was randomly presented to each child (using a balanced Latin Square) to avoid order effects. Table 9.1 shows the 11 actions and a brief description of each one. Each child was asked to come up with a gesture for each of the actions of the robot described in Table 1. For each action, Wobbrock's procedure was followed: (1) the robot performs a specific movement while the child observes, i.e. the referent, (2) the child is asked to perform any gesture or movement she wants so that the robot performs the referent action, i.e. the sign, (3) once the child starts to perform the gesture, the wizard (researcher) starts moving the robot according to the specific referent while following the child's gesture.

In contrast to Task 1, Task 2 and Task 3 required the children to perform a series of gestures over time in order to move the robot continuously following a predefined path on the ground marked with colored cardboards $(42 \mathrm{~cm} \mathrm{x} 59.6 \mathrm{~cm})$. All children were asked to perform both tasks, alternating the order between the participants to avoid ordering effects. The path in each task was designed to study the different modalities in which a child could give orders to a robot depending on the type of movement she wanted to perform. In Task 2, the path was formed 
Table 9.1: List of actions (referents) the mini-drone can perfom.

\begin{tabular}{r|c|l}
\hline $\begin{array}{r}\text { Action of the robot } \\
\text { (referent) }\end{array}$ & Code & Description \\
\hline Move forward & $\mathrm{F}$ & The drone advances forward \\
Move backwards & $\mathrm{B}$ & The drone advances backwards \\
Turn left & $\mathrm{L}$ & The drone turns left on-site \\
Turn right & $\mathrm{R}$ & The drone turns right on-site \\
Move left & $\mathrm{ML}$ & The drone turns left while advancing \\
Move right & $\mathrm{MR}$ & The drone turns right while advancing \\
Speed up & $\mathrm{SU}$ & The drone speeds up while it advances forward \\
Speed down & $\mathrm{SD}$ & The drone slows down while it advances forward \\
Jump & $\mathrm{J}$ & The drone performs a jump \\
Stop & $\mathrm{S}$ & The drone stops moving \\
Spin & $\mathrm{SP}$ & The drone turns around several times \\
\hline
\end{tabular}

by three straight segments, including one left turn and one right turn (see Figure $9.2)$.

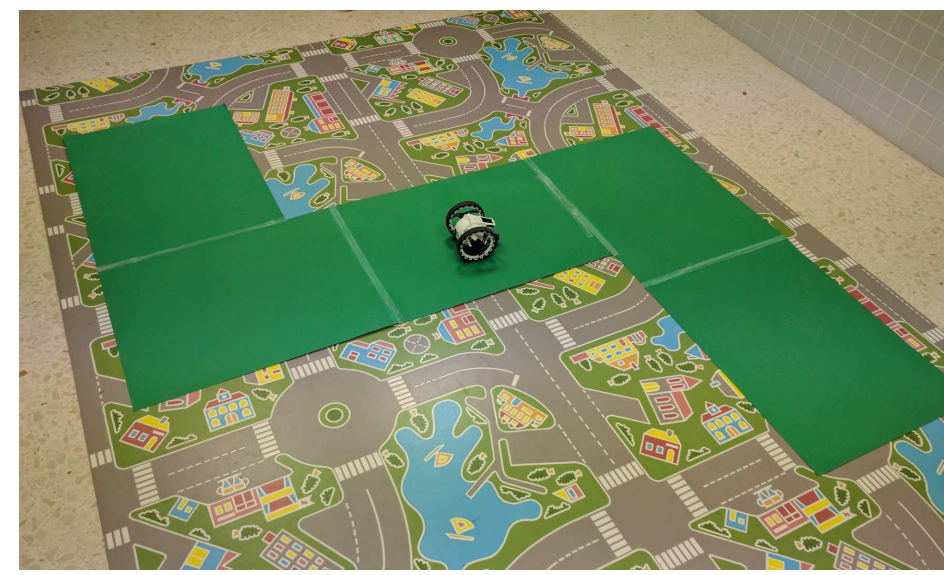

Figure 9.2: Path from Task 2.

Task 3 consisted of an irregular path, including one left turn, one right turn and three special cardboards (see Figure 9.3). On the blue cardboard, the children had to make the robot spin (SP) or jump $(\mathrm{J})$ and on the orange cardboards, they had to make the robot speed up (SU).

During Tasks 2 and 3, the children could perform gestures to give the robot any signs they wanted, for example, they could stop it and prevent it from leaving the path, or correct its trajectory if they thought that the robot might leave the path. 


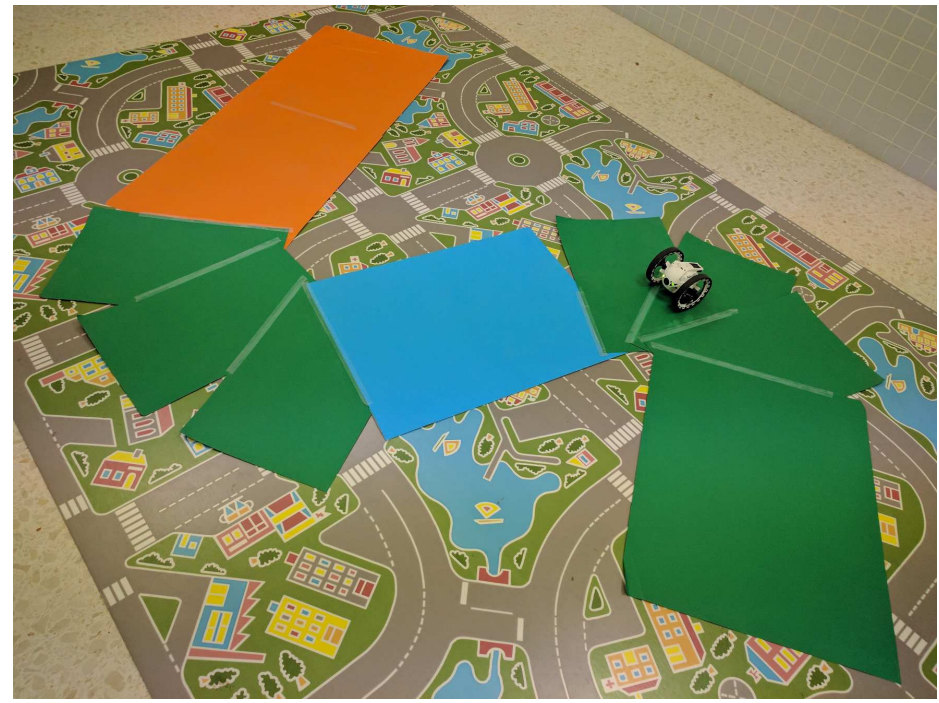

Figure 9.3: Path from Task 3.

After the three tasks, the children were asked to fill in a brief questionnaire [267] on their opinion of the activity, the control of the robot and potential improvements (see Table 9.2).

\subsubsection{Results}

\section{Time Performance}

Table 9.3 and Figure 9.4 sums up the average time the participants spent on each task by age group and gender. Start and end times for each task were computed from the time the researcher told the child that he had control of the robot to the time when the robot stopped moving after the child's last sign.

In the youngest age group (G1), the female participants spent on average more time than the male participants on each task. However, no statistically significant differences were found between genders in G1 according to the Mann-Whitney U test ( $\mathrm{T} 1: \mathrm{U}=41.000, \mathrm{p}=0.550 ; \mathrm{T} 2: \mathrm{U}=38.000, \mathrm{p}=0.411 ; \mathrm{T} 3: \mathrm{U}=25.000$, $\mathrm{p}=0.073)$. In $\mathrm{G} 2$, the girls spent on average more time than boys only on Task 1 , however there were no significant differences according to the Mann-Whitney $\mathrm{U}$ tests $(\mathrm{U}=31.000, \mathrm{p}=0.151)$. Male participants spent more time performing the activity in Tasks 2 and 3. No significant differences were found in Task 2 (U $=43.500, \mathrm{p}=0.622)$, but Mann-Whitney $\mathrm{U}$ tests showed statistically significant 
Table 9.2: Postquestionnaire and answer options.

\begin{tabular}{|c|c|c|}
\hline Code & Question & Answer Options \\
\hline Q1 & $\begin{array}{l}\text { How much did you } \\
\text { enjoy the game? }\end{array}$ & 5-point Likert scale \\
\hline Q2 & $\begin{array}{l}\text { How easy was it to } \\
\text { control the robot? }\end{array}$ & 5-point Likert scale \\
\hline Q3 & $\begin{array}{l}\text { Would you like to play with } \\
\text { the robot in school again? }\end{array}$ & Yes / No / Maybe \\
\hline Q4 & $\begin{array}{l}\text { Would you like to play with } \\
\text { the robot out of school again? }\end{array}$ & Yes / No / Maybe \\
\hline Q5 & $\begin{array}{l}\text { How would you like } \\
\text { to play again? }\end{array}$ & $\begin{array}{l}\text { Robot in the same room / } \\
\text { Robot in a different room }\end{array}$ \\
\hline Q6 & $\begin{array}{l}\text { Whom would you like } \\
\text { to play with again? }\end{array}$ & $\begin{array}{l}\text { Alone / Friends / } \\
\text { Family / Other }\end{array}$ \\
\hline Q7 & $\begin{array}{l}\text { What would you change in } \\
\text { the game to make it better? }\end{array}$ & Open answer \\
\hline
\end{tabular}

Table 9.3: Time performance (seconds) for each task by age group and gender.

\begin{tabular}{c|c|ccc}
\hline & & Task 1 & Task 2 & Task 3 \\
\hline \multirow{2}{*}{ G1 } & $\mathrm{F}$ & $\bar{x}=267.29, \sigma=71.679$ & $\bar{x}=23.86, \sigma=12.240$ & $\bar{x}=43.57, \sigma=16.772$ \\
& $\mathrm{M}$ & $\bar{x}=240.71, \sigma=54.109$ & $\bar{x}=18.00, \sigma=5.144$ & $\bar{x}=32.43, \sigma=15.210$ \\
G2 & $\mathrm{F}$ & $\bar{x}=224.40, \sigma=57.707$ & $\bar{x}=19.30, \sigma=5.774$ & $\bar{x}=23.60, \sigma=4.477$ \\
& $\mathrm{M}$ & $\bar{x}=191.00, \sigma=32.435$ & $\bar{x}=21.70, \sigma=9.141$ & $\bar{x}=35.70, \sigma=21.308$ \\
G3 & $\mathrm{F}$ & $\bar{x}=173.90, \sigma=19.393$ & $\bar{x}=23.20, \sigma=5,554$ & $\bar{x}=29.90, \sigma=11.406$ \\
& $\mathrm{M}$ & $\bar{x}=168.10, \sigma=28.544$ & $\bar{x}=18.30, \sigma=3.234$ & $\bar{x}=29.60, \sigma=10.426$ \\
\hline
\end{tabular}

differences by gender in Task $3(\mathrm{U}=23.5000, \mathrm{p}=0.044)$. In this case, the Boxand-Whisker plots for this task show two outliers in the male participant group, corresponding to two boys who spent considerably more time on Task 3 than the rest of their colleagues. In G3, the female participants spent more time on average on all tasks. While no statistically significant differences were found for Tasks 1 and 3 ( $\mathrm{T} 1: \mathrm{U}=41.000, \mathrm{p}=0.496$; $\mathrm{T} 3: \mathrm{U}=49.500, \mathrm{p}=0.9$ ), they were found between female and male participants in G3 for Task $2(\mathrm{U}=22.5000, \mathrm{p}=0.037)$. Although no outliers were identified in the Box-and-Whisker plots, this difference could be explained by analyzing the performance of the two female participants who took more time at this task. In both cases, the researcher performing the Wizard-of-Oz approach had difficulties understanding the intended meaning of the children's gestures. Nevertheless, considering the three age groups together, no statistically significant differences were found between the male and female 


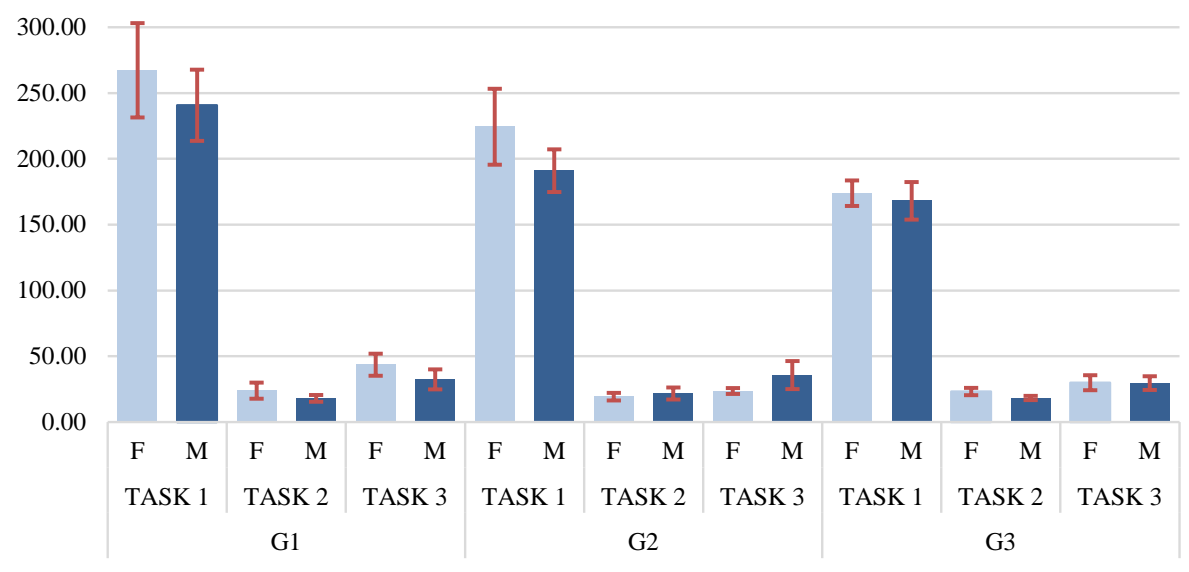

Figure 9.4: Time performance (seconds) for each task by age and group gender.

participants in any task, according to Mann-Whitney $\mathrm{U}$ tests $(\mathrm{T} 1: \mathrm{U}=415.500$, $\mathrm{p}=0.528 ; \mathrm{T} 2: \mathrm{U}=343.500, \mathrm{p}=0.093 ; \mathrm{T} 3: \mathrm{U}=440.500, \mathrm{p}=0.788)$

Mann-Whitney U tests were also performed to detect statistically significant differences between age groups in each task. There were no differences between age groups regarding the average time spent on Task 2 and Task 3. Statistically significant differences were found only in Task 1 between all groups: G1 and G2 $(\mathrm{U}=122.5, \mathrm{p}=0.022), \mathrm{G} 1$ and $\mathrm{G} 3(\mathrm{U}=30.500, \mathrm{p}=0.000)$, and $\mathrm{G} 2$ and $\mathrm{G} 3(\mathrm{U}$ $=89.000, \mathrm{p}=0.003)$.

\section{Gesture Taxonomy}

This section describes the taxonomy of gestures elicited from the study and the distribution of the observed gestures in the taxonomy by age group and gender. Table 9.4 sums up the gesture taxonomy derived from this study, which has been influenced by the taxonomies used by Wobbrock et al. [365], Karam and Schraefel [147] and Obaid et al. [227]. We manually classified all gestures in Task 1 according to the dimensions body parts, type and form, while Task 2 and Task 3 were used to classify the gestures according to the viewpoint dimension.

The children were free to perform any gesture or movement they wanted to for each action of the robot in Task 1. Hence, they could move anything from a hand to their whole body, e.g. walking besides the robot. Their elicited gestures have been analyzed regarding the body parts used to perform each action in Table 9.1 according to the taxonomy in Table 9.4. The three different categories in 
Table 9.4: Gesture taxonomy.

\begin{tabular}{|c|c|c|}
\hline \multirow{3}{*}{$\begin{array}{l}\text { Body } \\
\text { parts }\end{array}$} & One side & $\begin{array}{l}\text { The gesture is performed with one } \\
\text { hand, one arm or one leg }\end{array}$ \\
\hline & Two sides & $\begin{array}{l}\text { The gesture is performed with both } \\
\text { hands or both arms }\end{array}$ \\
\hline & Full-body & $\begin{array}{l}\text { The gesture is performed using the whole body, } \\
\text { or a combination of hand/arm and leg movements }\end{array}$ \\
\hline \multirow{4}{*}{ Type } & Deictic & $\begin{array}{l}\text { The children indicates the movement the robot has } \\
\text { to follow, either by pointing at its final destination } \\
\text { or by performing a dynamic movement indicating } \\
\text { the path/movement to be followed }\end{array}$ \\
\hline & Iconic & $\begin{array}{l}\text { The gesture visually depicts the movement to } \\
\text { be performed or is a visual metaphor of it }\end{array}$ \\
\hline & Mimicking & $\begin{array}{l}\text { The children imitates the } \\
\text { movement of the robot }\end{array}$ \\
\hline & Abstract & $\begin{array}{l}\text { The gesture has no direct mapping } \\
\text { with the robot's movement }\end{array}$ \\
\hline \multirow[t]{2}{*}{ Form } & Static & $\begin{array}{l}\text { After a short preparation phase in which the } \\
\text { user prepares the desired gesture, this } \\
\text { gesture does not entail movement }\end{array}$ \\
\hline & Dynamic & The gesture entails movement \\
\hline \multirow{2}{*}{ Viewpoint } & User-centric & $\begin{array}{l}\text { The gesture is performed from the } \\
\text { user's point of view (egocentric) }\end{array}$ \\
\hline & Robot-centric & $\begin{array}{l}\text { The gesture is performed from the } \\
\text { robot's point of view (allocentric) }\end{array}$ \\
\hline
\end{tabular}

which gestures can be classified attending to the body parts involved are: one side, two sides or full body interaction. As can be observed in Figure 9.5, most of the children used either one-side gestures or full body interactions, and this happened across all the different actions of the robot (see Figure 9.7). Gestures involving both sides of the body, such as using either both hands or both arms, were less frequent. According to the Pearson Chi-Square tests performed on the 662 gestures classified in Task 1, there is a statistically significant association between the participant group (children's age) and the preferred body parts they used to perform the gestures $(\chi(4)=46.222, \mathrm{p}=<.001)$. Post hoc analysis with adjusted Bonferroni correction showed that full body interactions were less frequent in children in G3 than in younger children in G1 $(\chi(2)=38.001, \mathrm{p}=<$ $.001)$ and $\mathrm{G} 2(\chi(2)=30.920, \mathrm{p}=<.001)$. In addition, children in G3 used both sides of their body statistically more frequently than children in G1 and G2 ( $\mathrm{p}=$ $<.001)$. There was also a statistically significant association between gender and the preferred body parts $(\chi(2)=10.265, \mathrm{p}=.006)$. In this regard, Figure 9.6 
shows that girls tended to use their whole body more frequently to interact $(43 \%$ of girls against $36 \%$ of boys), while boys tended to use just one side of their body ( $52 \%$ of the boys against $40 \%$ of the girls).

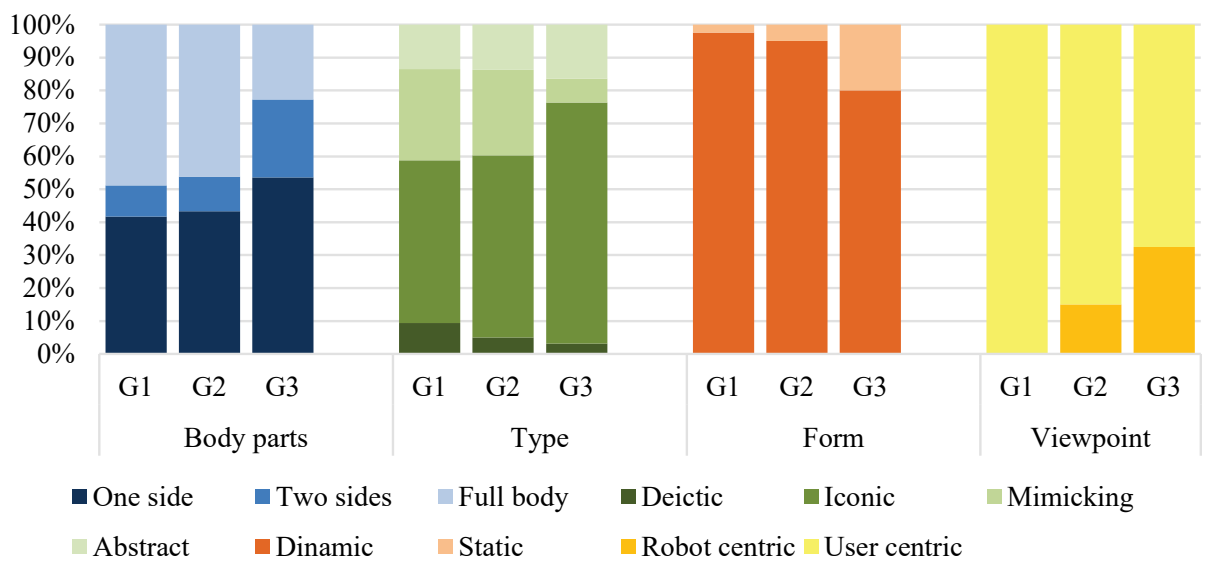

Figure 9.5: Taxonomy distribution according to age groups.

Another relevant aspect to consider within the elicitation study was the type of metaphors or abstractions the participants used in their gestures to give meaning to a specific action (or referent). This was somehow a new definition of a shared non-verbal language between the participant and the robot. One of the main goals of this study was to identify the type of gestures children would feel more natural to use when "speaking" to a robot by body language. The gestures were classified into four different types (see Table 9.4): deictic, iconic, mimicking and abstract. Deictic gestures are those in which children point with their fingers to a specific location or direction and may involve movement. In this case, the children point and trace the path the robot has to follow to perform a specific action. Iconic gestures are metaphoric gestures that imply either a direction, speed or visual depiction of the movement: moving the hand rapidly, moving the hand/arm from left to right, etc. Mimicking gestures are those in which children perform the same movement as the robot, as if they were showing the robot how to do it. Abstract gestures have no direct mapping with the direction, speed or movement of the action.

According to the Pearson Chi-Square tests performed on the data, there was a statistically significant association between the participant's age group and the type of gesture the children performed $(\chi(6)=48.014, \mathrm{p}=<.001)$. Post hoc analysis with adjusted Bonferroni correction showed statistically significant differences between G1 and G3 $(\chi(3)=44.253, \mathrm{p}=<.001)$, and between G2 and G3 $(\chi(3)=30.133, \mathrm{p}=<.001)$. Children in G3 usually performed more iconic 


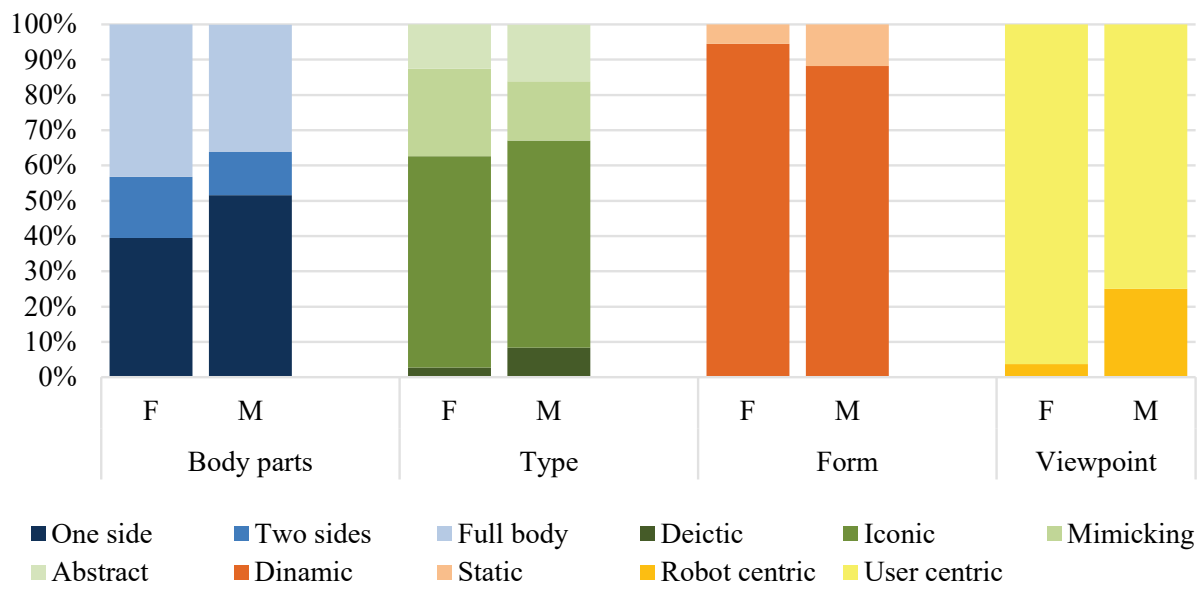

Figure 9.6: Taxonomy distribution according to gender.

gestures than children in G1 and G2 ( $\mathrm{p}=<.001)$. Mimicking gestures were rarely used by children in G3 ( $\mathrm{p}=<.001)$, while children in G1 and G2 made similar use of them. There was also a statistically significant difference between female and male participants regarding deictic gestures $(\chi(3)=15.506, \mathrm{p}=.001)$. Girls tended to use deictic gestures statistically less than boys did $(\mathrm{p}=.002)$, as can be observed in Figure 9.6. Figure 9.8 also shows the distribution of gesture types for the different actions and age groups.

The form of the gestures could be static or dynamic. Static gestures do not entail movement after an initial preparation phase in which the child places himself in the desired posture/gesture, e.g. horizontal still arm towards the front to indicate forward movement, as in Figure 8. Instead, dynamic gestures accompany the posture with movement, e.g. moving arm and hand from right to left to indicate movement in that direction, as in Figure 9. Figure 9.5 shows that children barely used static gestures and that all three age groups preferred dynamic gestures. However, the children in G3 used static gestures slightly more than the younger groups, and post hoc analysis with adjusted Bonferroni correction showed statistically significant differences between G1 and G3 $(\chi(1)=6.509$, $\mathrm{p}=0.011)$ in this regard. Girls used dynamic gestures $(94.44 \%)$ more frequently than boys $(88.24 \%)$ on average. However, there was no statistically significant difference between genders, according to Pearson Chi-Square tests $(\chi(1)=1.415$, $\mathrm{p}=0.234)$.

The viewpoint dimension was a very interesting aspect to evaluate. According to the taxonomy, a robot-centric gesture is one in which the sign is given according to the robot's coordinate system, e.g., the robot has to move towards the left 


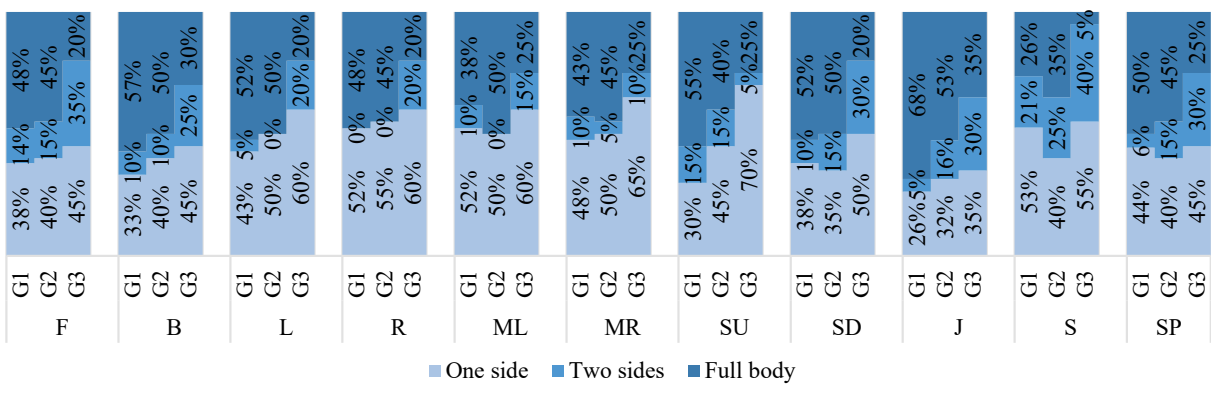

Figure 9.7: Distribution on the use of body parts for different actions by age group.
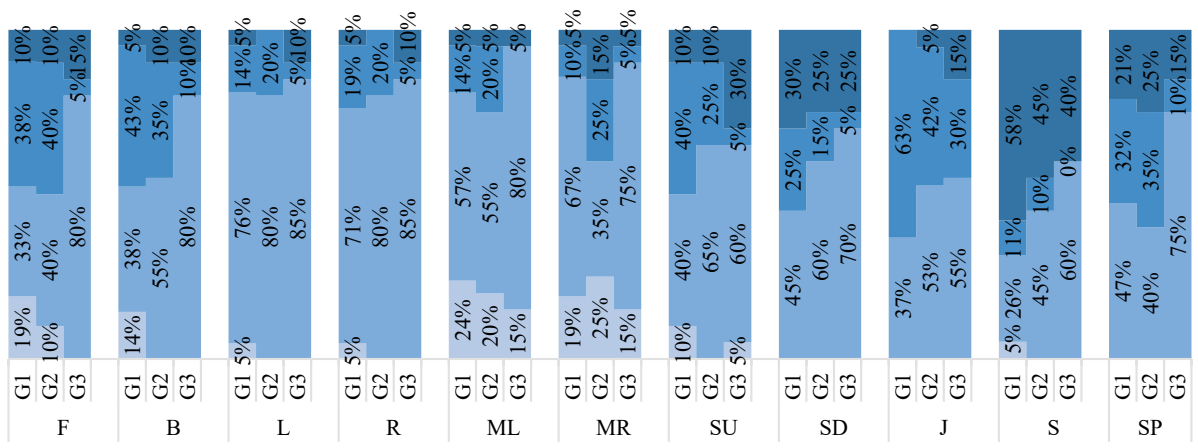

$\square$ Deictic $\square$ Iconic $\square$ Mimicking $\square$ Abstract

Figure 9.8: Distribution of types of gestures by action and age group.

side of the room and it is already facing the left wall, hence the user performs a Forward gesture such as in Figure 8. On the other hand, user-centric actions are given according to the user's coordinate system, regardless of the orientation of the robot, as in Figure 9.9b. The referent actions provided in Task 1 started always with the robot in the same position and orientation, and it was difficult to assess whether children were considering a user-centric or a robot-centric point of view, as both coordinate systems mapped to each other in this initial configuration. In order to assess children's preferences towards robot-centric or user-centric gestures, we manually classified this dimension in Task 2 and Task 3 for each child (122 samples). The nature of tasks 2 and 3 was different from the elicitation activity in Task 1 as the referents in Task 2 and 3 were not discrete actions of the robot. Instead, in Tasks 2 and 3 the children had to move the robot over a predefined path on the ground. This allowed us to observe clearly which coordinate system they adopted as reference for their interactions, as well as whether they changed the 
gestures they were performing in Task 1 to new ones better suited to the nature of these new tasks.

Overall, the children usually adopted a user-centric view, with an increase in the adoption of a robot-centric view with age. According to the Pearson ChiSquare tests performed on the data, there was a statistically significant association between the participant's age group and the viewpoint the children adopted $(\chi$ $(2)=16.473, \mathrm{p}<.001)$. Post hoc analysis with adjusted Bonferroni correction showed statistically significant differences between G1 and G2 $(\chi(1)=6.797$, p $=0.009)$ and between G1 and G3 $(\chi(1)=16.222, \mathrm{p}<.001)$. This confirms that the observations in Figure 9.5 are statistically significant: no children in G1 used a robot-centric view, while $15 \%$ of the children in G2 and $33 \%$ of children in G3 preferred this viewpoint instead of a user-centric one. In relation to gender, on average girls adopted a user-centric view (96.30\%) more frequently than boys did $(75.00 \%)$. In this regard, Pearson Chi-Square tests confirmed a statistically significant difference between female and male participants regarding the adopted viewpoint $(\chi(1)=10.382, \mathrm{p}=0.001)$.

Children were free to use the gestures they wanted for Tasks 2 and 3, even if those gestures were different from the ones they had elicited during Task 1. Children usually performed the same or very similar gestures between Task 1 and Task 2, and between Task 1 and Task 3, with only $34.43 \%$ of children changing most of the gestures they had defined in Task 1 in these two new activities. However, once they had established these new gestures for either Task 2 or Task 3, children kept those gestures the same between Tasks 2 and 3 , with only 5 children changing again the set of gestures between tasks.

\section{Gesture Language}

This section describes the gestural language elicited from the children's gestural data collected during Task 1 of the study for controlling a ground mini-robot. The selection of a suitable gesture for each control action took into account the agreement levels among the children's defined gestures. The process of selecting a suitable gesture for a control action is as follows:

- For each control action $a$ we identify a set $P_{a}$ that contains all the proposed gestures.

- The proposed gestures in $P_{a}$ are then grouped into subsets of conceptually identical gestures $P_{i^{1 \ldots N}}$, where $i$ is a subset that contains conceptually identical gestures and $\mathrm{N}$ is the total number of identified subsets. A conceptually identical gesture can be either the same identical gesture, or a gesture that entails the same movement and meaning, e.g. moving left hand from left to 


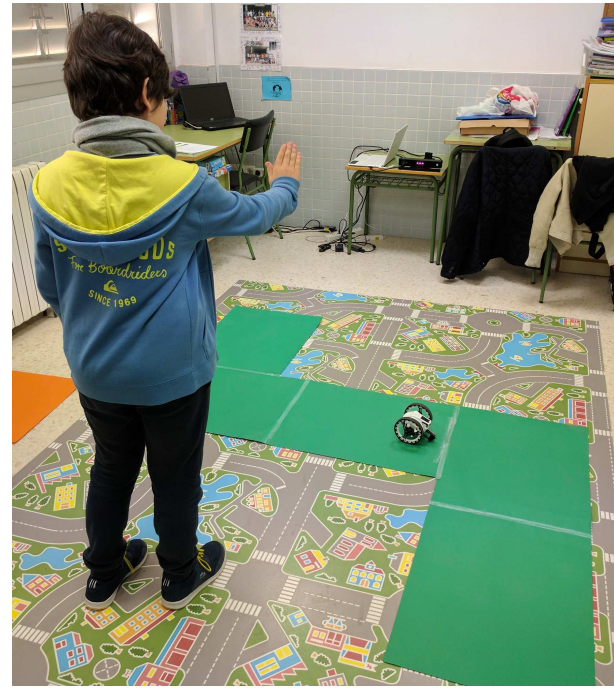

(a) Robot-centric gesture.



(b) User-centric gesture.

Figure 9.9: Example of robot-centric and user-centric gestures.

right would be conceptually identical to moving left hand with extended left arm from left to right.

- The representative gesture for the control action $a$ is identified by selecting the largest subset $P_{i}$.

To further evaluate the degree of agreement among the participants regarding the selected user-defined sets, we followed the process proposed by Wobbrock et al. $[333,334]$ and used AGATe software to compute an agreement score for each control action.

Table 9.5 reports the agreement levels for each action by participant group. The gestures with the highest agreement rates among all the participants were Jump $(J)$, Stop (S), Spin (SP), Forward (F) and Backward (B). On the other hand, agreement rates for gestures involving turns (L, R, ML, MR) and speed changes (SU, SD) were quite low. However, it was observed that for those actions with low agreement rates, there were two or three gestures with similar frequencies instead of a single preferred gesture. For example, for ML and MR actions, around 15-23\% of the time the children chose to move their hand or arm from left to right (or right to left), while $16-20 \%$ of the time they chose to draw a curve in the air. By considering the two or three most frequent gestures for each action, we were able to provide a consistent and robust gestural language that conforms to almost $50 \%$ 
of the participants in the three age groups. The most frequent gestures for each action are illustrated in Figure 9.10 with their respective frequencies.

Table 9.5: Agreement level of gestures for the different actions proposed in $\mathrm{T} 1$ by participant group.

\begin{tabular}{ccccc}
\hline Action & G1 & G2 & G3 & All groups \\
\hline F & 0.176 & 0.142 & 0.111 & 0.125 \\
B & 0.205 & 0.179 & 0.153 & 0.155 \\
L & 0.057 & 0.089 & 0.042 & 0.070 \\
R & 0.052 & 0.147 & 0.042 & 0.081 \\
ML & 0.138 & 0.053 & 0.089 & 0.098 \\
MR & 0.067 & 0.053 & 0.042 & 0.061 \\
SU & 0.186 & 0.100 & 0.084 & 0.104 \\
SD & 0.076 & 0.053 & 0.037 & 0.048 \\
J & 0.390 & 0.337 & 0.195 & 0.309 \\
S & 0.071 & 0.184 & 0.263 & 0.161 \\
SP & 0.090 & 0.189 & 0.147 & 0.133 \\
Average & 0.137 & 0.139 & 0.110 & 0.122 \\
\hline
\end{tabular}

\section{Postquestionnaire Results}

After the three tasks, children were asked to complete a postquestionnaire about their experience and thoughts. The questions are reported in Table 9.2, and this section outlines the main results.

Regarding question Q1, all the children in G1 and G2 and almost all those in G3 reported that the activity with the robot was "Great". Only three children in G3 did not select the highest option in the Likert scale, however they also reported a very positive experience. Overall, the activity seemed to be very amusing and enjoyable for the children (see Figure 9.11). According to the Mann-Whitney U test, there were no statistically significant differences between age groups (G1-G2: $\mathrm{U}=199.5, \mathrm{p}=1.000$; G1-G3: $\mathrm{U}=178.5, \mathrm{p}=0.069 ; \mathrm{G} 2-\mathrm{G} 3: \mathrm{U}=161.5, \mathrm{p}=$ $0.083)$. In addition, there were no significant differences by gender in any age $\operatorname{group}(\mathrm{G} 1: \mathrm{U}=49.0, \mathrm{p}=1.000 ; \mathrm{G} 2: \mathrm{U}=45.0, \mathrm{p}=1.000 ; \mathrm{G} 3: \mathrm{U}=35.0, \mathrm{p}=$ $0.067)$.

The children were also asked about the perceived easiness of controlling the robot (Q2). Most of them reported that it was extremely easy (see Figure 9.12), especially those in G1 (aged 6-7 years old). None of them found it difficult and only one child from G2 ranked this task as having a normal difficulty level. The Mann-Whitney $\mathrm{U}$ test showed no significant differences by gender in any age group (G1: $\mathrm{U}=45.5, \mathrm{p}=0.480 ; \mathrm{G} 2: \mathrm{U}=43.5, \mathrm{p}=0.891 ; \mathrm{G} 3: \mathrm{U}=43.0, \mathrm{p}=$ 

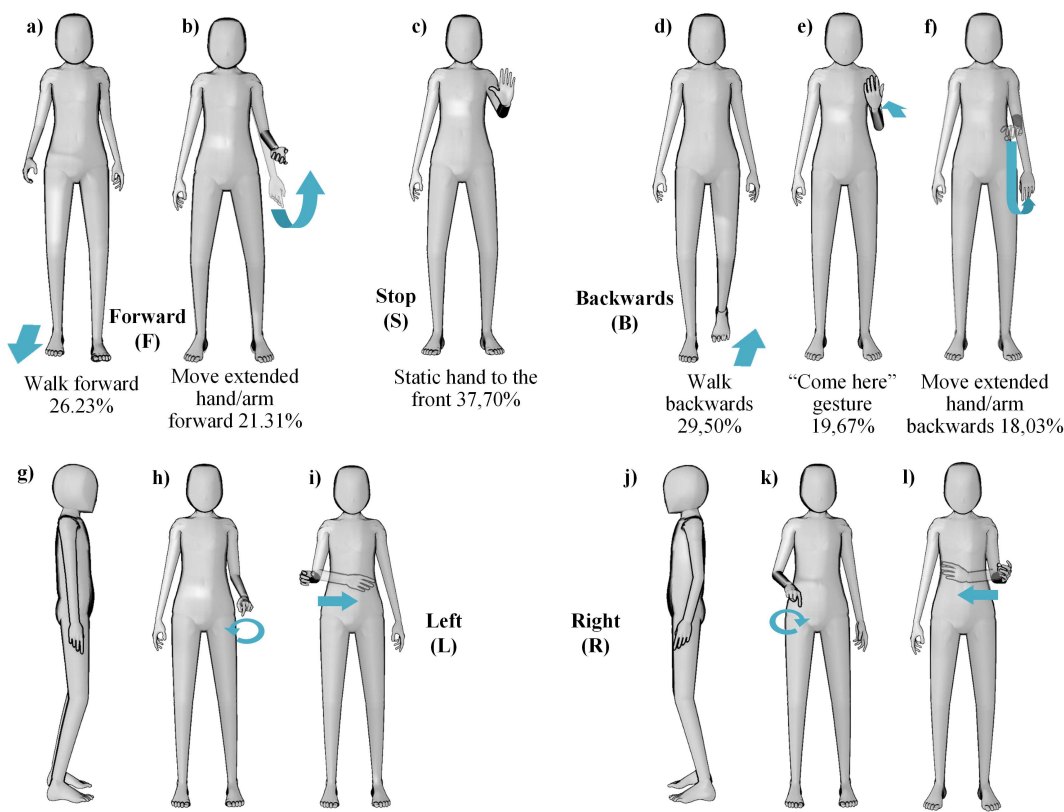

Quarter turn

Draw circles Move hand/arm left $16.39 \%$
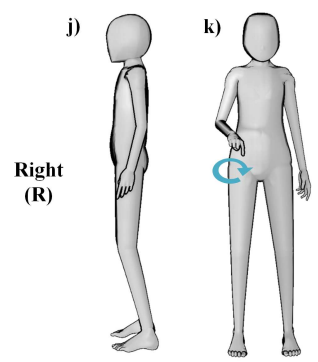

l)
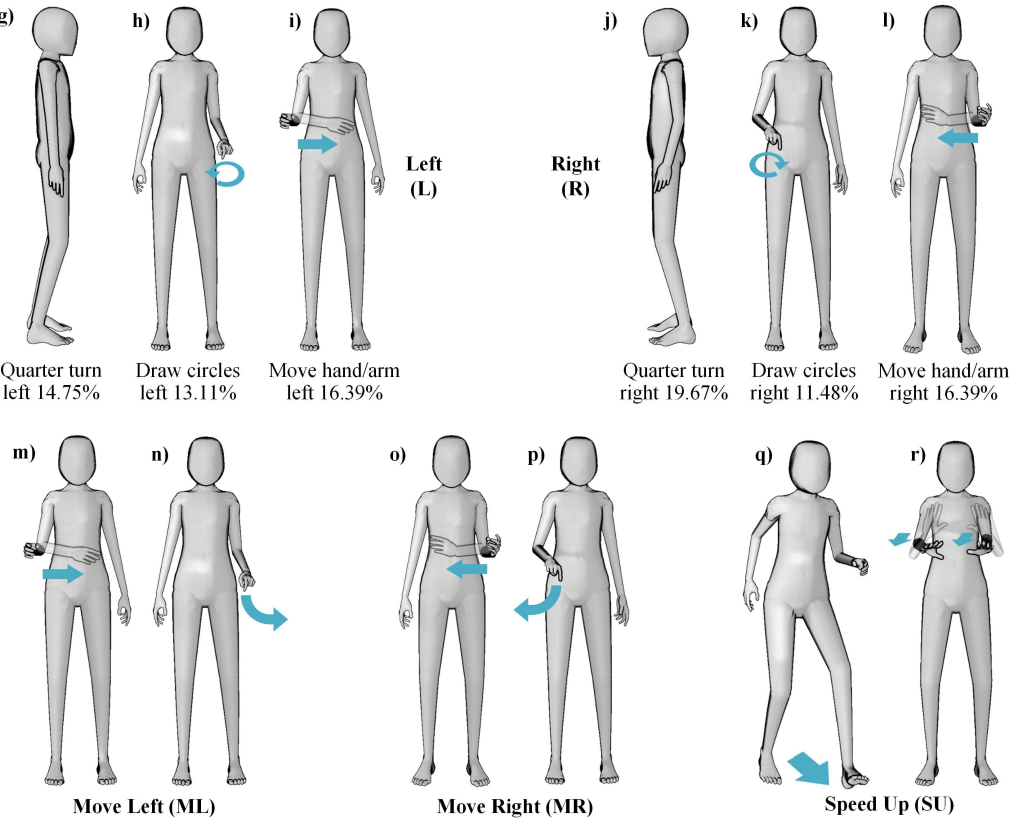
$\begin{array}{rrr}\text { Quarter turn } & \begin{array}{c}\text { Draw circles } \\ \text { right } 19.67 \%\end{array} & \begin{array}{r}\text { Move hand } / \text { arm } \\ \text { right } 11.48 \%\end{array} \\ \text { right } 16.39 \%\end{array}$

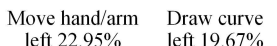

left $22.95 \% \quad$ left $19.67 \%$

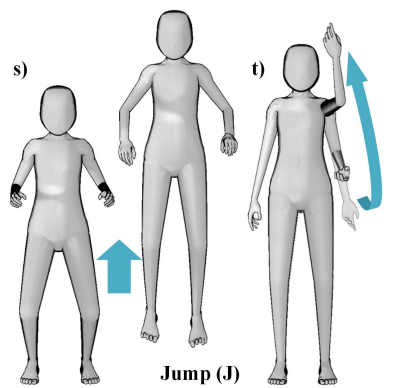

$\begin{array}{cc}\text { Jump } & \text { Move extended } \\ 44.26 \% & \text { hand } / \text { arm up } 34.43 \%\end{array}$
Move hand/arm Draw curve right $14.75 \%$ right $16.39 \%$

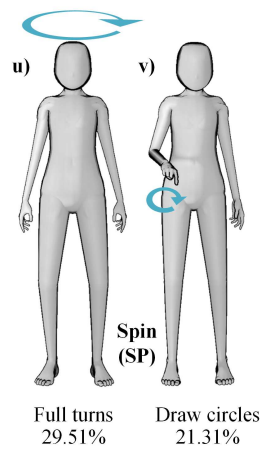

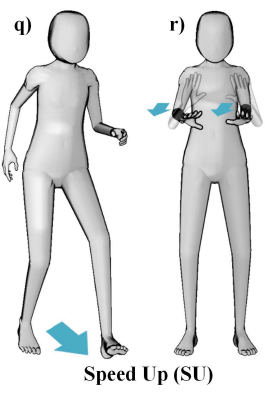

Run forward Push arms

$22.95 \%$ forward $16.39 \%$

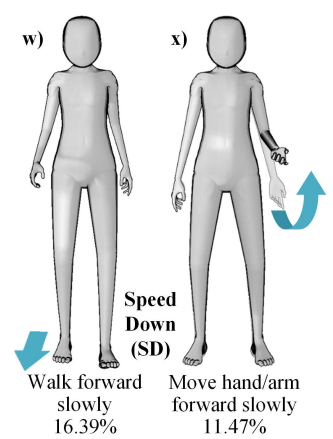

Figure 9.10: Gestural language with most frequent gestures for each action and occurrence frequencies. 
How much did you enjoy the game?

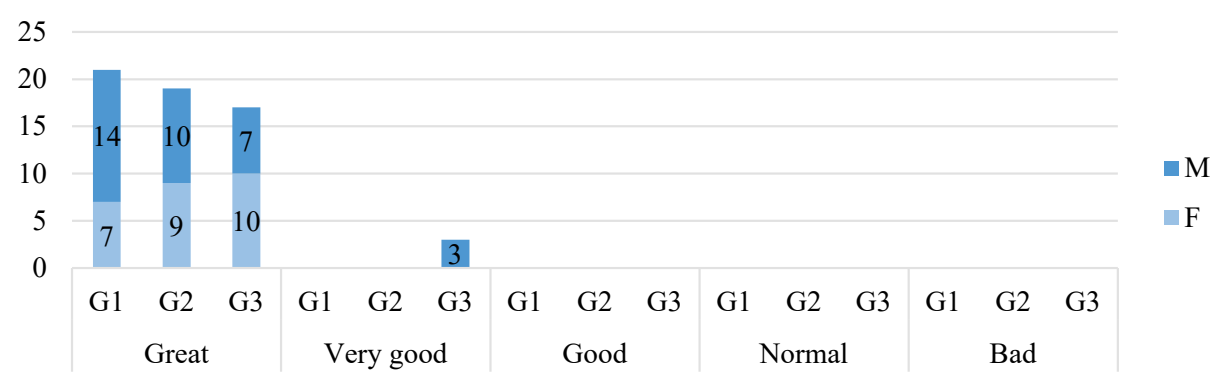

Figure 9.11: Results for Q1 in the postquestionnaire by participant group and gender.

0.565). Regarding age groups, Mann-Whitney U test revealed statistical significant differences between $\mathrm{G} 1$ and $\mathrm{G} 2(\mathrm{U}=113.5, \mathrm{p}=0.002)$ as well as between $\mathrm{G} 1$ and G3 $(\mathrm{U}=103.0, \mathrm{p}=0.000)$.

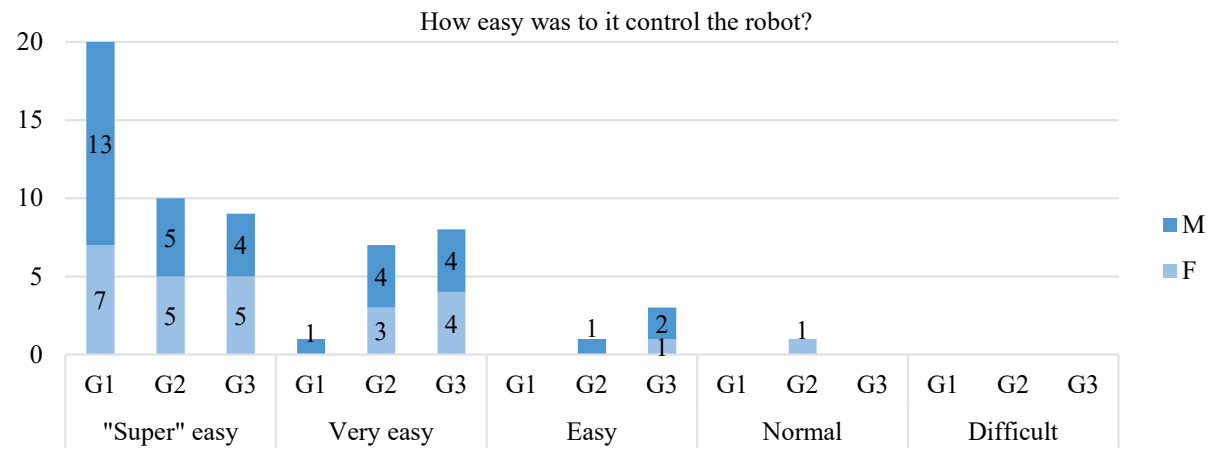

Figure 9.12: Results for Q2 in the postquestionnaire by participant group and gender.

Another important aspect to consider was whether the children would like to play with the robot again and in which contexts. Figure 9.13 and Figure 9.14 show that they were very enthusiastic about playing with the robot again either in or outside the school, without significant differences. In addition, the robot used in the experiment had a camera that allows the user to observe on a screen what the robot is seeing in real time. This supports remote control of the robot: the user could give commands to the robot without having visual contact with it, but could guide it thanks to the built-in camera. This interactive scenario would be really useful for situations in which children cannot leave their physical location, as in the case of long-term hospitalized children. Hence, Q5 was aimed towards 
exploring the acceptance of children towards this kind of remote activity in order to design such a playful experience. The two interactive modalities with the robot, i.e. on-site vs. remote, were explained to all children in the questionnaire in order to ask them whether they would like to play with the robot again in the same room, control it remotely from a different room, or both (Q5). Figure 9.15 and Figure 9.16 show that most of the children were curious about exploring this kind of remote interaction, mentioning that in this way they could spy on their friends while they are in classroom, or they could use the robot to move things around and bring them to another person. The children in all the groups either preferred to play again only with the remote version, or marked both modalities, which opens a promising path towards the development of remote interactive systems for children based on NUIs.

Would you like to play with the robot in school again?

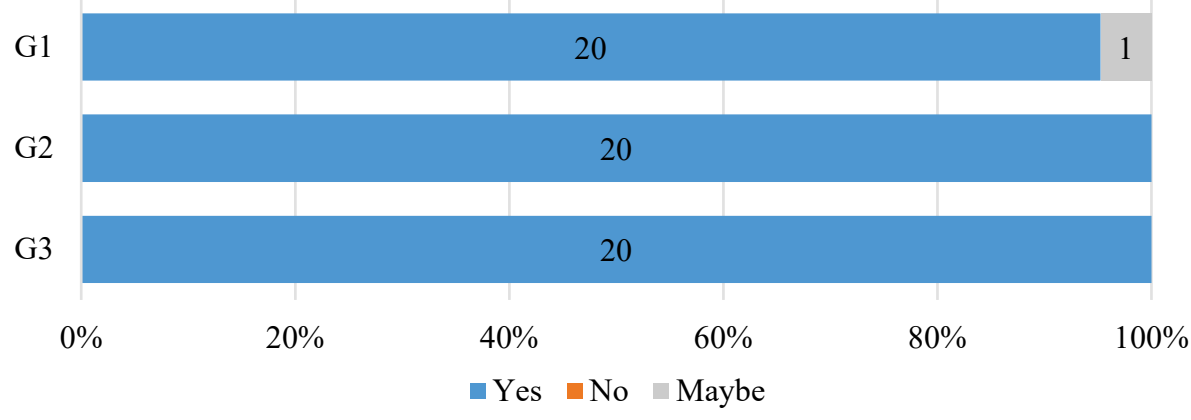

Figure 9.13: Results for Q3 in the postquestionnaire by participant group.

Would you like to play with the robot out of school again?

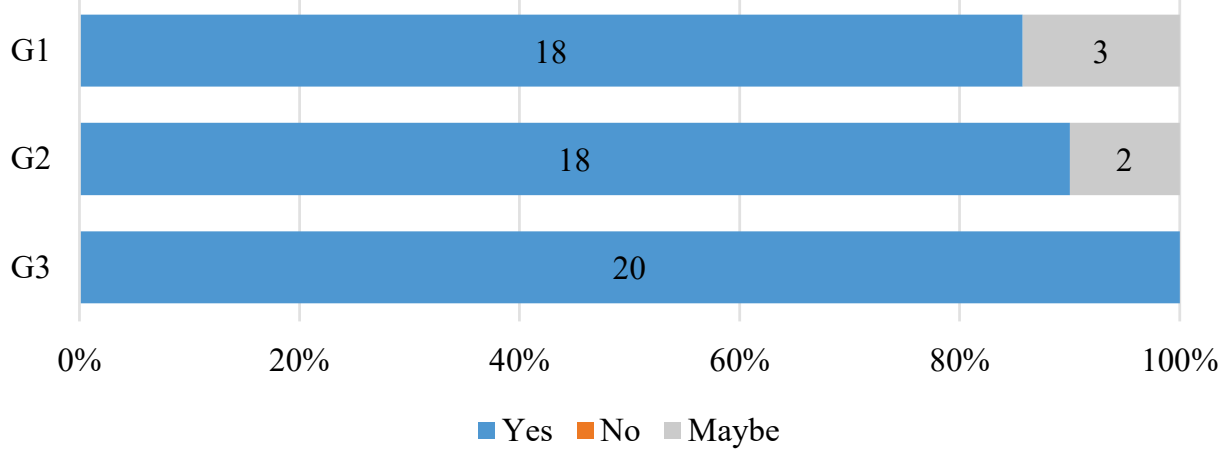

Figure 9.14: Results for Q4 in the postquestionnaire by participant group. 


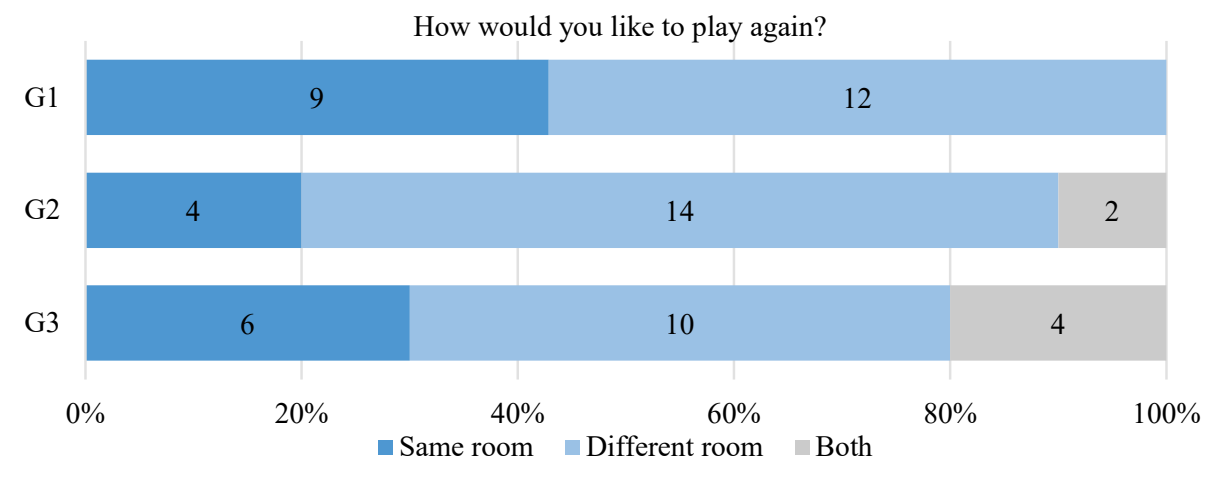

Figure 9.15: Results for Q5 in the postquestionnaire by participant group.

Another promising opportunity for gesture-based control of mini-robots would be to create collaborative activities that foster educational values such as collaboration, communication, trust, etc. For these reasons, the children were asked whether they would like to play alone or with other people, such as friends, family or others (Q6). They were able to choose several options in this question. Figure 9.17 shows the histogram of each answer per participant group and gender. As can be observed, the shape of the distributions by age group and by gender are similar. Most of the children reported they would like to play with their friends, family and even others (instructor, teacher, etc.). The ones who preferred to play alone were mostly boys and only two girls preferred this option. However, only six out of these 10 children chose the solo option only, while the remaining four indicated they would also like to play with their friends or family.

At the end of the session, children could also propose any changes that they would like to incorporate to the game or the robot (Q7). Several children reported that they would love to have several robots and be able to play with their friends. Some children commented they would like the robot to have more actions, and others asked for bigger or more complicated paths as well as incorporating obstacles.

\subsection{Discussion and Future Recommendations}

The study explored the comprehensive set of research questions defined in Section 3 with the aim of advancing research and improve the development of future gestural languages for children depending that are suitable to their capabilities and preferences. This section will review the defined research questions in Section 3, providing recommendations for future designs. 
How would you like to play again?

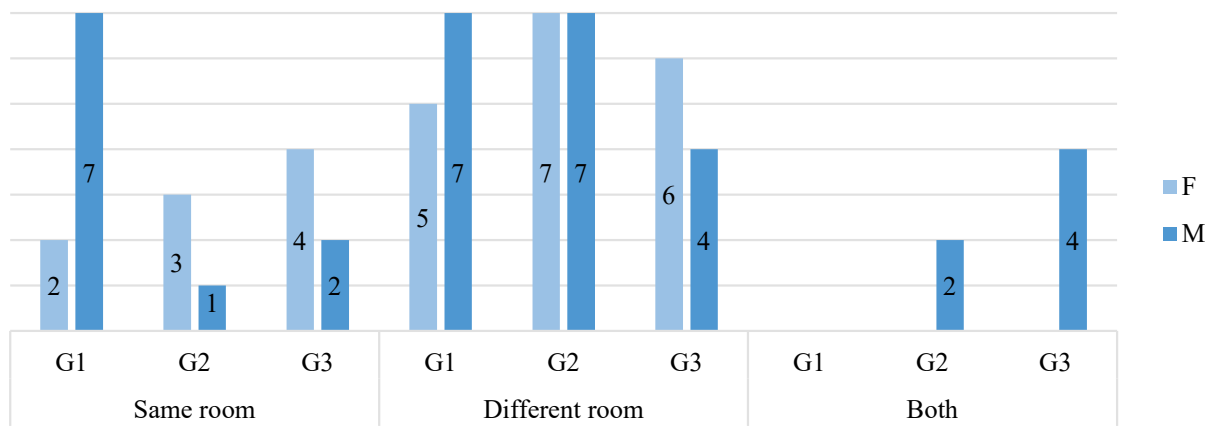

Figure 9.16: Results for Q5 in the postquestionnaire by gender and participant group.

Whom would you like to play with again?

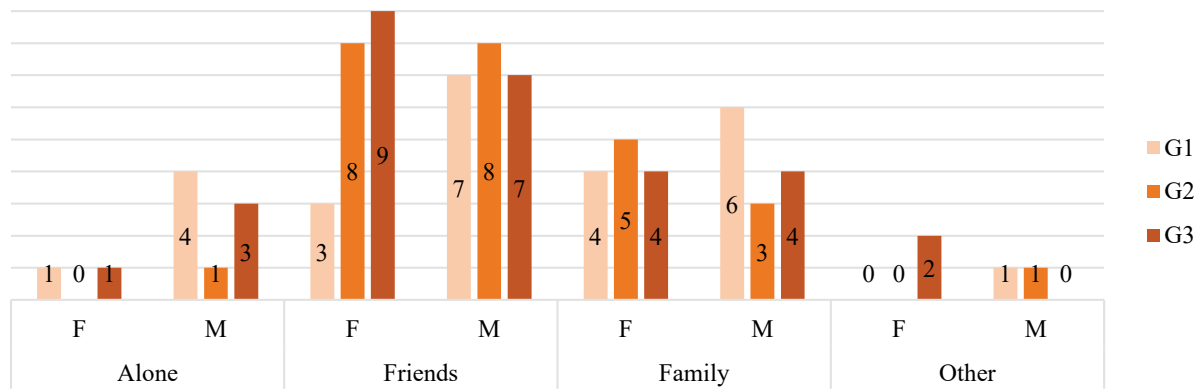

Figure 9.17: Results for Q6 in the postquestionnaire by participant group and gender.

The answer to RQ1 (whether there are time performance differences by gender) is negative. Although there were differences in G1 and G3, they were explained by either communicative issues in the Wizard-of-Oz approach or outlier participants. Moreover, considering the three age groups together, the time performance using gestural communicative interactions with robots was similar for all the participants, regardless of gender. This is a promising result as it allows us to define a gestural language that could be used by a large population.

Regarding RQ2 in Section 3.4.1, significantly statistical differences regarding time performance were found in Task 1 between all age groups. However, these results are still positive as no differences were found in Tasks 2 and 3, which took place later. It seems that all the participant groups required different lengths of 
time to become familiar with the robot and the gesture signs they were defining, which can be explained by differences in cognitive skills relative to their age [242]. However, once they got used to the device after Task 1, all the groups exhibited a comparable time performance in Tasks 2 and 3, which involved controlling the robot and giving it signs to move over a predefined path. In this regard, finding a suitable gestural language would mean that children of different ages in the 6-12 age range could perform equally well if provided with the appropriate communicative metaphors, in spite of their different cognitive and motor skills.

The elicited gestures were analyzed according to the taxonomy in Table 9.4 in order to answer RQ3 (whether age and gender influenced the kind of gestures according to the taxonomy). In this regard, several differences were found. For example, younger children preferred mimicking gestures while older children tended to produce iconic gestures instead. This is also related with the younger children's preferences for full-body gestures, because these full-body gestures usually mimic the robot's movements. In addition, the form and viewpoint of the elicited gestures was also affected by age. Overall, the children clearly preferred dynamic gestures and a user-centric viewpoint. However, as they get older there is an increase in the percentage of static gestures and they start adopting a robot-centric viewpoint more frequently. This latter effect has also been observed in other studies with children [49, 243]. Regarding gender differences, girls used fewer deictic gestures than boys, but adopted a user-centric viewpoint more frequently than their male counterparts. This could be due to gender differences in the abstraction of space and previous exposure to technological activities that entail spatial representations, such as playing videogames or controlling a robot $[312,337]$. In addition, boys preferred to use just one side of their body to interact, while girls tended to use their whole body.

Despite the differences by age and gender, the set of gestures in Figure 9.10 that emerged from the elicitation study can allow for all the aforementioned differences. In this respect, the answer to RQ4 is affirmative, as we proposed a gestural language that provides agreement across gender and age. Implementing the two or three most common gestures for each of the proposed actions would allow to account for the different communicative modalities found. For example, either mimicking or iconic movements could be performed for most actions. This could support progressive change from mimicking gestures to iconic ones due to age differences. Regarding body parts, we could implement each of the iconic gestures in our gestural language with two versions: one hand/arm or two hands/arms. In this way, each action would have a full-body gesture as well as gestures that can be performed with one or two sides of the body. This would cover a wide range of preferences depending on age and gender, as shown in RQ3. Considering the form, less than $20 \%$ of the elicited gestures were static, and most of those happened in the preferred gesture for the Stop $(\mathrm{S})$ action, which has been included in the gestural language. Therefore, most of the gestures in the proposed language 
would be dynamic. The speed of the movement would also be considered in order to allow for actions such as SD, which consists of similar, but slower, gestures as F. Perhaps the most complicated decision would be whether to use a user-centric or a robot-centric viewpoint. Our findings are consistent with the results of [49, 243], which described how children gradually change from egocentric to allocentric gestures over time. In the age range considered in this study, the majority of participants used a user-centric viewpoint even in the oldest age group. However, if we also want to adapt to the robot-centric viewpoint preference, a possible solution is to have an initial "analysis" phase before the real interaction starts. This phase would consist of simply asking the child to move the robot along a predefined path, as in Task 2. Automatic analysis of the child's gestures during this initial test would allow classifying the user within the taxonomy. With this information on the user's intuitive preferences, the system could give more weight to a specific set of gestures among the ones in the proposed language. For example, if we detect that the user prefers a user-centric viewpoint with full body interactions in the analysis phase, the viewpoint would be set to user-centric while gestures a) and d) would have more relevance than gestures b), e) and f) (see Figure 9.10). It remains to be studied whether the increase in robot-centric viewpoint preferences continues to rise in children over 12 years old.

Answering the research question that considered the impact of age and gender in the perceived enjoyment of the robot (RQ5), the results show that there are no differences and that overall enjoyment was rated very highly.

Regarding differences in the perceived easiness of the elicitation task (RQ6), the results showed no differentiation by gender. In terms of age differences, although age group G1 rated this aspect slightly more positively than G2 and G3, the overall assessment was extremely positive. These positive results confirm that the Wizard-of-Oz approach provided the desired effect in the participants, allowing the children to perform the gestures that came most naturally to them. As the children were defining their own gestures, it was also expected that they found it easy to control the robot if the Wizard-of-Oz was correctly carried out. These results will be compared with future results of children using the gesture language, once it has been implemented in the robot. Using these results as a ground base will help to decide whether the implementation of the proposed gesture language suits children's expectations.

The motivation to play was also reported as very positive, without significant differences by gender or age (RQ7). Almost all the children reported they would like to play with the robot again, either at school or outside school. The fact that they would like to play with the robot again even outside school could indicate that they perceived this activity as a playful experience. Powerful interactive scenarios for learning could therefore be developed by making use of this intrinsically motivating technology. 
Regarding RQ8 (whether robots can be considered as a promising tool for remote playful activities), the answer is affirmative. The postquestionnaire answers to Q5 shown in Figure 9.15 and Figure 9.16 demonstrate that children are motivated to use the robot in a remotely controlled scenario. This triggered their curiosity towards exploring areas out of their sight, and imagining fun scenarios in which they could use the robot to spy on their classmates, etc. This aspect, in combination with the social component of collaborative playful scenarios with robots, could help to create highly motivating learning activities.

In response to RQ9 (whether robots can be considered as a promising tool for collaborative playful activities), the results of the postquestionnaires showed very positive perceptions (Q6, see Figure 9.17). This motivates the design and development of collaborative scenarios between children, or even between a child and his parents/teachers. Such scenarios could use the gesture-based control of a robot as a tool towards pursuing a collaborative goal that promotes educational and social values.

Another interesting aspect that emerged during the study was that the children were so immersed in the activity that they frequently forgot about the tracking technology. In other cases, even though they had been introduced to the tracking device at the beginning of the experiment, several children thought the tracking system was in the robot's camera. Consequently, they sometimes placed themselves not facing the Kinect sensor in order to interact directly with the robot. There is thus a need for interactive spaces with several sensors capable of detecting and responding to variable and natural movements of the children who are not aware of where the tracking mechanisms are while they play.

Comparing the children's elicitation study for drone interaction in this manuscript to previous work in gesture elicitation for drone interaction with adults [43, 226], it can be observed that both children in this study and adults in previous works [43] tend to consider the drone as an animate being, sometimes even speaking to it as if it was a pet. In addition, children performed a more varied set of gestures to interact with drones than adults in previous studies $[43,226]$. While adults usually performed gestures using just one or two hands/arms [43, 226], children had a divided preference between those gestures and full body interactions. Adults do not usually move their whole body to mimic the robot's movements, while when designing for children this should be an essential gestural communicative modality. 


\subsection{Threats to Validity}

Although this study obtained very interesting results, several precautions must be taken before generalizing these results to other contexts. Despite the fact that the elicitation study is intended to allow children to perform any kind of gesture that feels more natural to them, previous background may condition their responses. For example, children who already own a drone/robot or who have previous experience with videogames, robotics, etc. may have developed their own spatial representations and semiotics. However, this is hard to isolate in current elicitation studies, as children of this age range are now highly exposed to technology.

In addition, the experiment involved only one interactive robot. The physical attributes of the robot could also have played a role in the specific gestures the children performed. For example, some of the children commented that the robot resembled a pet, and the robot was fitted with a camera that clearly indicated its orientation. Hence, the performed gestures could also have been influenced by these attributes, so that using a different robot, e.g. a spherical one with no marked orientation, may produce a different set of gestures.

We believe all these threats to validity could be addressed by performing a new set of experiments to validate the proposed gestural language with different types of ground robots.

\subsection{Conclusion and Future Work}

This paper describes the first gesture elicitation study with children using full body interactions to control tangible ground robots. The children's preferred gestures and body language communication with robots in interactive spaces were identified. This has resulted in the definition of a gestural language that accounts for the outlined variabilities of such gestures by age group and gender, which also showed a good acceptance rate in the studied age range. The children were found to be highly motivated towards the activity and the easiness of the interaction was rated as very positive. The next step will be the validation of the proposed gestural language in terms of accuracy of the gesture tracking, usability and learning curves for children with a Kinect sensor.

The encouraging results obtained from the questionnaires revealed that interactive spaces with robots using body gestures could become promising collaborative scenarios. We have now started a study in which pairs of children collaborate in controlling a ground mini-robot to achieve a common goal. The results of this study are expected to provide further information on collaborative learning activ- 
ities with robots using full-body interaction. Moreover, the children's perceptions of the easiness and social aspects of the activity will also be evaluated.

A further aspect that emerged from the postquestionnaires was the children's curiosity about remote interaction. In this respect, we are designing an interactive space for hospitalized children who might not be able to leave their room, such as those in pediatric transplant or oncology wards. Remotely controlling a robot could help these children to explore spaces outside their room or even socialize with other children or adults, thus helping to generate positive emotions during their stay and foster relationships and communication with their teachers/parents/companions. Although animal therapy is often used in hospitals to improve the patients' wellbeing, children in oncology wards cannot usually benefit from such an experience due to their condition. For this reason, this will be an interdisciplinary study in which children could use the proposed gestural language to remotely control a robot to play with an animal located outside the hospital. We believe the intuitive nature of controlling the robot by gestures and the ability to play with an animal in real-time will greatly benefit the mental wellbeing of young patients in these difficult situations.

\section{Acknowledgments}

This work is funded by the European Development Regional Fund (EDRF-FEDER) and supported by the Spanish MINECO (TIN2014-60077-R). The work of Patricia Pons is supported by a national grant from the Spanish MECD (FPU13/03831). Special thanks are due to the children and teachers of the Col-legi Públic Vicente Gaos for their valuable collaboration and dedication. 


\title{
Chapter 10
}

\section{Designing interspecies playful interactions: studying children perceptions of games with animals}

Patricia Pons, Javier Jaen

ISSI Group, Department of Computer Systems and Computation (DSIC),

Universitat Politècnica de València, Valencia, Spain

Published in Proceedings of the Fourth International Conference on Animal Computer Interaction, pp. 12:1-12:12, (2017). doi:10.1145/3152130.3152139

\begin{abstract}
While animals' playful interactions with technology are gaining attention, little research within Animal Computer Interaction has addressed how humans' would like to participate in such games. This paper presents a study in which children designed interspecies games between humans and animals, with and without technological artifacts. The resulting designs helped to start understanding how children perceive and want to participate in such playful interventions. By means of semiformal interviews, drawings and questionnaires, the factors that might be shaping their designs have been outlined. In addition, the design activity in itself revealed as a promising tool to foster critical thinking and unveiled potential educational activities aimed at strengthening children relationships with different
\end{abstract}


animal species. The resulting designs also serve as groundwork to develop playful interventions between children and animals.

\subsection{Introduction}

The field of Animal Computer Interaction (ACI) [173, 177] has observed a significant growth of studies focusing on animals' playful interactions with technology [5, $78,254,258,260,350,361]$. Play is an innate and natural activity that has emerged as a mechanism within ACI to include animals as designers of the animal-centered technology ACI pursues [142, 253, 349, 352]. Play can also generate enjoyable experiences, leading to the improvement of animals' wellbeing [363]. In fact, playful interactions with technology can foster cognitive and physical stimulation for non-human animals, but also for humans as well [131, 216, 217]. In this regard, technologically mediated games between humans and non-human animals have the potential to foster relationships and strengthen bonds between humans and animals in several ways.

While ACI is already studying how animals would like to participate in playful scenarios [76, 247, 349, 352, 361], few works have addressed human perceptions about their participation in such playful contexts. If we aim to design playful systems for animals and humans so that their shared experiences can create bonds, both the animals' and humans' needs have to be taken into account. Following a user-centered approach on both ends of the interaction would allow creating a playful experience that is meaningful for all the players. Hence, both animals and humans should be active participants and co-designers of the playful interactions.

The design of playful scenarios can also help to raise awareness on good or bad practices about the use of technology with animals. Pet owners, zoo visitors, or humans willing to interact with an animal might not even be aware of the benefits technologically mediated games can provide to either domestic or non-domestic animals. It is therefore essential that we understand what human users envision when we talk about technologically mediated games with animals. In addition, humans' perceptions when designing the system would allow us to detect misconceptions, ethical issues and learning opportunities. This is even more important to consider in young generations who will shape and use future digital systems. Hence, children participation in the design and use of an interspecies playful activity with animals might be a promising tool to foster critical thinking and improve their relationships with different species.

This work consequently presents a study in which hospitalized children had to design two interspecies games between a human and an animal of a given species. The aim of this work is to analyze how children perceive the animal participant, its abilities and participation in the game based on its species, and study the 
different factors that affected how children shaped the game and the technology for the proposed scenario. The outcomes of this paper are manifold. Firstly, the way in which children relate and empathize with different animal species affects their social cognitive developments. Therefore, this design activity and the children reflections over their designs would help to strengthen the relationships between children and animals. Second, studying children perceptions about different animal species helped to detect whether different educational activities might be needed. Finally, animals have been widely used in hospitals to improve the wellbeing of patients based on the positive effects they evoke. However not all patients are able to interact with therapy animals. Focusing this design activity on hospitalized children allowed to identify which kind of technological games could be created for them so that they can remotely interact with an animal, helping to improve their wellbeing during their hospital stay.

This paper firstly describes several works regarding human perceptions of animal technology. Then, the design study and results are reported, followed by a discussion about the results and future opportunities. Finally, the main conclusions and immediate future work are summarized.

\subsection{Related Works}

\subsubsection{Participatory Design with Children}

Participatory design $[207,208]$ with children $[58,59]$ has already been the focus of discussion within the field of Child Computer Interaction (CCI). Including children as co-designers helps to create more suitable and engaging final products for them. Many works have addressed ways to include children as active designers of the systems they are going to use [73, 109, 126, 287]. Several works have identified that children do not feel comfortable with video cameras [57], while they do like to use cut outs and drawings to represent their thoughts $[109,266]$. In addition, a semiformal structure along the cooperative design process is recommended [101], while the activity should be fun and as short as possible [266].

Due to the novelty of the ACI research field, there are no previous studies regarding children participation in the design of technologically mediated games for animals, nor their attitudes towards such games. Therefore, this work proposes the use of participatory design techniques with children for such goal. 


\subsubsection{ACI Playful Interactions and Human Perceptions}

Related studies within ACI have focused on adults' viewpoints of animals using several types of technology. The Kinecting with Orangutans project [343] has examined zoo visitors' perceptions of orangutans using an interactive system. Using a projector and a Microsoft Kinect sensor outside the orangutans' enclosure, researchers were able to track the orangutans' interactions over digital elements projected on the ground of their enclosure. Interviews with zoo visitors revealed that by watching the orangutans physically interact with the technology, humans showed cognitive, emotional and motor empathy. The emergence of diverse degrees of empathy towards a species provides a promising framework for the creation of engaging interspecies encounters.

The authors in [117] discussed how data gathering within ACI studies usually relies on information provided by the animals' owners. However, owners do not always have sufficient background regarding animal behavior. Therefore, the information they provide to the researcher might not always be reliable. By providing dog owners with a behavioral information sheet, results showed that the information gathered from the owners was $33 \%$ more reliable as they were better informed for providing contextual information about their dogs' emotion. These results evidence how a better-informed human user would help to create better animal-centric systems.

The work in [17] stresses the need for careful use of tablets with animals and the importance of humans being responsible and aware of the kind of technology they give to their pets. The authors identified several types of dog-tablet playful interactions, in which the observed behaviors ranged from enjoyment to overstimulation. The need for careful use of tablets raised awareness on the possible dangers that could emerge if owners do not understand the impact of such interactions. As a follow up work, the authors studied human attitudes towards dogs using tablets [18]. Their preliminary results indicate that preexisting opinions on dog-tablet interaction may influence one's judgement. Therefore, more efforts should be done in understanding human perceptions of technology for animals. This would facilitate the ideation of better plans for reshaping biases and misinterpretations, which would eventually help humans to design and provide better technological scenarios for their animals.

Another study has focused on the perceptions of designers towards ants after a workshop session in which the designers had to create "escape rooms" for the insects [353]. The designers showed changes in their perceptions and attitudes towards the insects during the workshop, such as increased sensitivity and interest. Moreover, Jorgensen and Wirman [142] state that humans' participation as co-designers of an animal's playful activity with technology promotes the development of more inclusive and suitable games. We believe this is even more important in the case of interspecies games in which the human would also participate in the 
activity. Hence, this paper situates children in the role of co-designers and future players of interspecies games with animals. Through the proposed design activities, this study aims to highlight how children envision such games, unveil their perceptions towards different animal species and analyze how to raise awareness on the importance of considering animals as equal participants.

\subsection{Designing Games for Animals}

\subsubsection{Participants}

Participants were hospitalized children from Hospital Universitari i Politècnic La Fe in Spain. The hospital has its own school for the pediatric wing and attends children from primary and secondary school. In this study participated 28 children (14 boys and 14 girls), with ages from 5 to 13 years old (Mean $(\mathrm{M})=9$, Standard Deviation $(\mathrm{SD})=2.037)$. The sessions were conducted during teaching hours of the school.

There were two groups of participants. The first group (NT) conducted the activities proposed in the study without any restrictions in the design ( 6 boys and 7 girls, ages from 6 to $13, \mathrm{M}=9.69, \mathrm{SD}=1.932$ ). The second group ( $\mathrm{T}$ ) had only one condition: they should use at least one technological artifact in each of their designs ( 8 boys and 7 girls, ages from 5 to $12, \mathrm{M}=8.40, \mathrm{SD}=1.993$ ). They were given a set of technological devices they could use, but they were free to propose new ones or invent new features and affordances. The following subsection will explain in detail the procedure and materials used in the study. These were the same for both participant groups, with the only difference that group $\mathrm{T}$ had to include the technological aspect in their designs.

\subsubsection{Procedure and Materials}

Each child performed the activity individually with the researcher. The sessions were of approximately 20-30 minutes depending on the child level of detail of the explanations and pictures. Each child was asked to design two games, each game with a different animal.

For a game design, the child was given a cutout of a girl or a boy, meant to represent herself, and a cutout of the animal participating in the game. In addition, plenty of cutouts of other children, adults and animals of several different species were available on the table for their use in case they would like to add any other human and non-human animals to the scenario (see Figure 10.1). A white A3 sheet was given to the child for each design. They could glue the cutouts they 




Figure 10.1: Cutouts used in the design activities.

wanted to the design sheet, and they could also draw anything they wanted to use in the game with color pencils.

For each design, the child fills in a brief prequestionnaire about the animal, previous knowledge and feelings. Then, the design activity began and the child could draw, glue and use anything available on the table to represent the game on the white sheet of paper. During this activity, the researcher conducted a semiformal interview with the child in order to understand the game and guide the design process. The audio of the interviews was recorded and analyzed after the sessions. This allowed the researcher to focus on the child without taking notes during the interviews. It also created a more comfortable space for the children. Some of the questions asked by the researcher to guide the design were: Where are you? Where is the animal? How will you play? What will you do? What will the animal do? After the child finished the design, they answered a brief postquestionnaire. 
Table 10.1: Classification of animals presented in the study.

\begin{tabular}{r|cccccc}
\hline & Quadrupeds & Felids & Anthropocentric & Aquatic & Winged & Reptiles \\
\hline $\begin{array}{r}\text { Domestic } \\
\text { Wild }\end{array}$ & Dog & Cat & Child / Adult & Fish & Bird / Parrot & Snail / Lizard \\
& Tiger & Gorilla / Orangutan & Dolphin & Owl / Eagle & Snake / Crocodile \\
\hline
\end{tabular}

\subsubsection{Experimental Design}

The animals for which the children designed games are classified in Table 10.1. This classification was not meant to be extensive nor cover all kind of animals. Instead, it includes animals that children might be familiar with, such as domestic animals or animals which can be usually seen at the zoo or TV. This personalized taxonomy divided the animals in six different categories attending to their behavior, anatomy and "likeability". Each child designed two games for two animals in the same category, i.e., dog and elephant, fish and dolphin, etc. This allowed to study whether there were any differences between games for domestic/small animals and animals usually seen in zoos or in the wild.

The prequestionnaire was meant to assess the initial knowledge and feelings about the animal before designing the game. It was comprised of four open-answer questions and seven Likert questions (see Table 10.2 and Table ), of which five are shared with the postquestionnaire. Both the prequestionnaire and the postquestionnaire had a common section with five questions (see Table 10.3) inspired in the Fun Toolkit questionnaire [266]. These shared questions allowed to study whether the perceptions of the children regarding the animal changed after the design and elicit the reasons for those changes. Table 10.4 shows the remaining questions of the postquestionnaire. Four of them were open-answer questions intended to make the children reflect on how their design could be improved and think about how the animal would enjoy or perceive the game. However, they were free to leave them blank if they did not have any ideas or thought their design was adequate. There were also three Likert questions to assess whether they thought the game would be fun for both animals and humans, and if they were interested in knowing more things about the animal after the activity.

Table 10.2: Questions before the game design activity.

\begin{tabular}{c|c|c}
\hline & Prequestionnaire & Code \\
\hline & Have you seen this animal before? Where? & PQ1 \\
Open answer & What does he eat? & PQ2 \\
questions & Where does he live? & PQ3 \\
& How does he play? & PQ4 \\
\hline $\begin{array}{c}\text { 5-point Likert } \\
\text { questions }\end{array}$ & Do you think this animal likes to play? & PQ5 \\
\hline
\end{tabular}


Table 10.3: Questions to be asked before and after the game design activity.

\begin{tabular}{c|c|c}
\hline & Shared questions & Code \\
\hline & How much do you like this animal? & SQ1 \\
& Do you think it is beautiful? & SQ2 \\
5-point Likert & Do you think it is friendly? & SQ3 \\
questions & Do you think it is intelligent? & SQ4 \\
& Does it scare you? & SQ5 \\
\hline
\end{tabular}

Table 10.4: Questions after the game design activity.

\begin{tabular}{|c|c|c|}
\hline & Postquestionnaire & Code \\
\hline \multirow{4}{*}{$\begin{array}{l}\text { Open answer } \\
\text { questions }\end{array}$} & $\begin{array}{l}\text { Do you think you could change something in the } \\
\text { game to make it more fun for the animal? How? }\end{array}$ & TQ1 \\
\hline & $\begin{array}{l}\text { Do you think you could change something in the } \\
\text { game to provide more physical exercise to the an- } \\
\text { imal? How? }\end{array}$ & TQ2 \\
\hline & $\begin{array}{l}\text { Do you think you could change something in the } \\
\text { game to make it easier to use/understand for the } \\
\text { animal? How? }\end{array}$ & TQ3 \\
\hline & $\begin{array}{l}\text { Do you think you could make the animal learn } \\
\text { things with your game? How? }\end{array}$ & TQ4 \\
\hline \multirow{3}{*}{$\begin{array}{l}\text { 5-point Likert } \\
\text { questions }\end{array}$} & $\begin{array}{l}\text { Do you think you will enjoy playing with the an- } \\
\text { imal to this game? }\end{array}$ & TQ5 \\
\hline & $\begin{array}{l}\text { Do you think the animal will enjoy playing with } \\
\text { you to this game? }\end{array}$ & TQ6 \\
\hline & $\begin{array}{l}\text { Do you want to know more things about the an- } \\
\text { imal? }\end{array}$ & TQ7 \\
\hline
\end{tabular}

\subsubsection{Observational Findings}

This section reports some general relevant aspects regarding the use of technology in the two participant groups. The first group of participants (NT) were free to design any kind of game they would like, without mentioning if they should or should not include technology within the game and how. This degree of freedom allowed observing that children did not naturally include technology in games designed for and with animals. They rather designed situations that they are accustomed to see in TV documentaries or at the zoo, or even used their own past experiences with a specific animal to design the game. Only two children in this group included technology in their designs: P10 depicted two children playing a 
videogame console, and $\mathrm{P} 7$, who might not have understood well the activity in the first place, designed a tablet-based game in which one of the characters was a snail.

Children in the NT group were asked after the two designs whether they would consider including technology as part of the game. None of them seemed convinced about including technology within the design, either because they could not envision how this could be done or because they expressed concerns about the animal breaking the technology or being unable to use it. For instance, P1 designing for a cat and a tiger commented: "Perhaps with a tablet... but the cat would break the robot. And the cat cannot use the tablet. A robot for cats could be invented." After this, P1 described a possible gym for cats using machines and objects to encourage them to chase the objects, jump and exercise. However, when asked about this idea being used for tigers, P1 was not sure that it could work well in such case. P4 and P8, designing for birds, were also asked about the use of technology. P4 commented that using a drone may cause that the bird fights with it. P8 said that it would be very difficult for a bird to use a tablet, and the animal could eventually break it. Regarding the use of robots, P8 mentioned that the bird "would grab it but it would break it as well". Therefore, it was observed from the interviews that most of the children in NT group showed certain degree of sensitivity towards the suitability of the technology regarding the animal species being considered. However, P7 in the NT group said that the animals (snake and snail) could play a tablet-based game, without thinking much about the physical traits of the animal nor showing interest in explaining how would that game be. Besides, P10 said that he would play a videogame with a gorilla, describing the gorilla as an anthropomorphic friend.

By contrast, children in the second group (T), who were specifically asked to choose technological devices for the game, showed two different approaches. On one hand, there were children who considered the animal as a friend, sometimes even taking an anthropomorphic view of the animal and did not express those concerns during the design of the game (same as happened with P10 in the NT group). On the other hand, there were children who carefully revised the different technological devices proposed by the researcher trying to choose the most appropriate one for the given animal. The technology chosen by the children as well as the game dynamics they designed were based on different aspects the children considered important, as can be observed in the designs, such as the animals' physical traits, their habitual location and behaviors, etc. 


\subsubsection{Design Results}

In this section, we present several aspects that emerged after reviewing the designs and interviews with the children following a thematic analysis with the data. Several examples of the games they envisioned are also reported.

\section{Physical Location and Physical Boundaries}

Both groups of participants did not depict any physical boundary between them and the animal in the case of domestic ones, except for two: a parrot drawn within a fence in a zoo enclosure, and a fish in a fish tank. The majority of the designs for the domestic category showed the animals in non-restricted areas such as the house, a park, a yard, the sea, etc., and the children placed themselves close to the animal.

Regarding wild animals, the designs showed a different situation. In nine of the 26 designs with wild animals, the animal was depicted inside a zoo enclosure and the child was located on the other side of the fence or physical barrier they had drawn (see Figure 10.2a). Interestingly, only two of those designs corresponded to the group in which technology was used, while the other seven drawings were from the NT participant group. In the remaining 17 designs, there was no physical boundary and the animal was depicted in a naturalistic environment. Two different contexts were identified: (1) In five of those designs, the child was in the same scenario as the animal without any physical division but the game was designed in such a way that either the child was hidden or the animal was motivated to move away from the child (see Figure 10.2b). Usually, this latter case involved the use of a technological artifact the child was managing, driving it away from the animal: a race between a snake and an $\mathrm{RC}$ car, a crocodile who had to follow a drone carrying meat, or a gorilla following a small robot. (2) In twelve of those designs, the animal was envisioned as a friend capable of understanding the child's intentions and the game, without any concerns about danger: gorilla and child racing to get a banana, tiger listening and dancing with a musical robot, owl and child playing hide-and-seek, or eagle playing friendly with a drone. Figure 10.3 presents a summary of these findings.

\section{Animals' Physiological Traits}

It was observed that in many designs, especially the ones involving technology, the children tried to match the physical traits and abilities of the animal to the dynamics of the game and the selection of the technology to be used.

Within the 26 designs from the NT participant group, 24 of them depicted an interaction between the child and the animal that either replicates behaviors they 


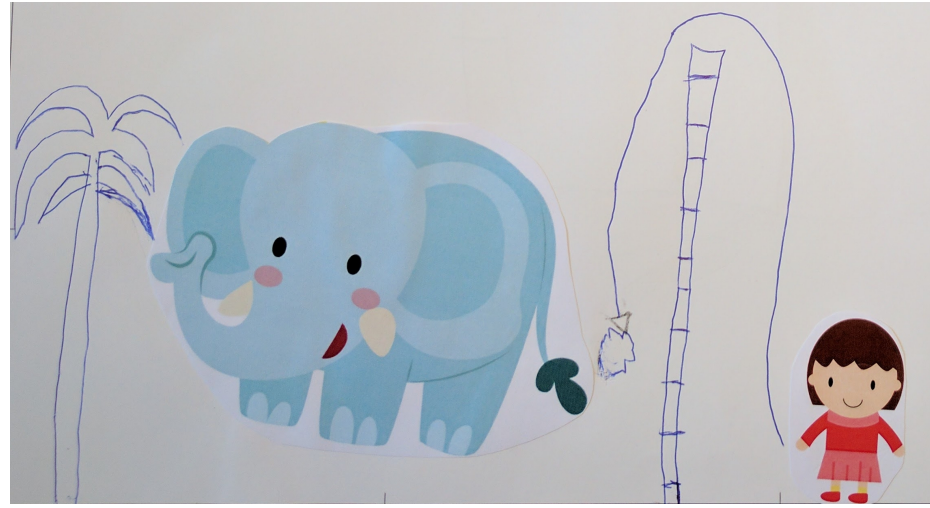

(a) Child feeding an elephant at the zoo with a physical fence between them.

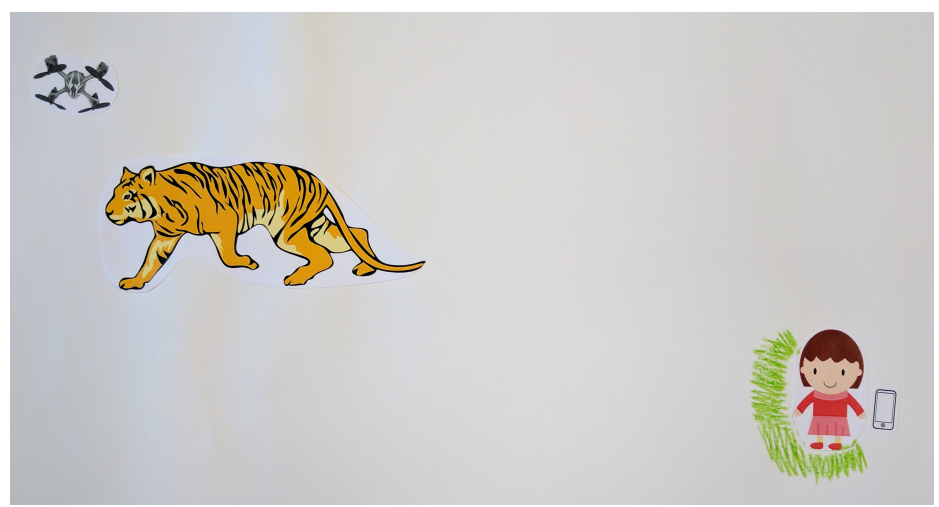

(b) Child hiding from tiger in the wild while controlling a drone.

Figure 10.2: Examples of different physical locations and boundaries.

usually see happening with that animal (14), or that match the animals' physical traits with the goal of the game (10). In the first case, many games involved wild animals depicted in zoos, and the interaction was feeding the animal or giving it commands (feed an elephant, give vocal instructions to a tiger, and ask a dolphin to go through a ring). However, the same could be observed also with domestic animals, which were depicted in naturalistic scenarios being fed by the child, e.g., bird, owl, fish, or playing common games such as fetching an object, e.g., cats and dogs. In the latter case, some designs were a race between a crocodile and a child in two separated pools, a race between a gorilla and a child climbing up a tree to get a banana, or a child taking a walk mounted on an elephant.

Regarding the $\mathrm{T}$ participant group, sixteen of their designs matched the technological artifact with the physical restrictions the animal could have when using it. 


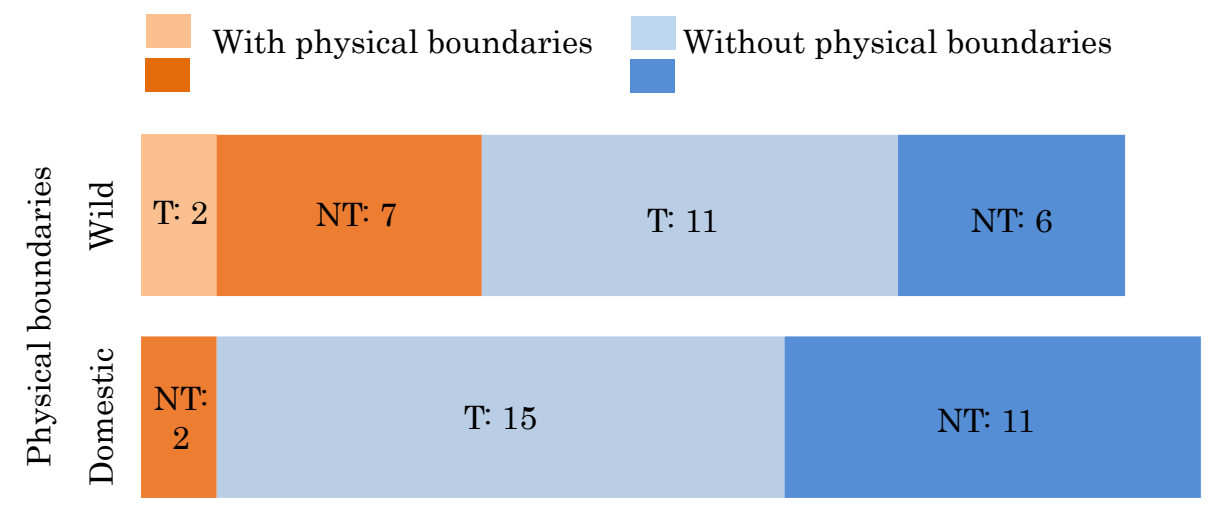

Figure 10.3: Physical boundaries in the designs.

For example, tablets or mobile phones were avoided, as the animals might not be able to use them with their paws, beaks or hands. Instead, the children preferred to use RC cars or small ground/aquatic robots for the non-flying animals (Figure 10.4a), while choosing drones to interact with flying ones (see Figure 10.4c). Some examples: cats, dogs, a snail, a tiger and even a gorilla who had to chase a small robot controlled by the child, two birds who had to follow a drone controlled by the child, and fish playing with small robots that could work under water or over the sand at the bottom of the sea. The remaining ten designs involved technology that might not match properly with the animals' characteristics. For example, three designs were about birds chasing a ground robot controlled by the child. Two designs used tablets, one for a parrot and the other one for a lizard. In the first one, the child acknowledge that the parrot will not be using the tablet; instead, the child would select and play videos on the tablet so that the bird could watch them together. The lizard design was inspired by an Internet video the child saw, in which a frog tries to eat ants from a tablet-based game. He proposed to help the lizard by also touching his own tablet in a collaborative fashion. It turned out that the food aspect was a recurrent topic also in the $\mathrm{T}$ participant group. Two designs, one for a tiger and another one for a crocodile, proposed to use a drone controlled by the child that the animal had to chase. In the crocodile design (Figure $10.4 \mathrm{~b}$ ), the drone would be carrying meat so that it would be a feeding exercise for the animal. In the tiger game (Figure 10.2b), the drone was not carrying meat initially, however during the postquestionnaire the child proposed such addition in an attempt to make the game more appealing for the animal.

Regarding the four designs, two from each participant group, concerning children as the other species participating in the game, the proposed games were 




Figure 10.4: a) Race between a snake and RC car, b) Child using a drone to fed the crocodile, c) Eagle playing with drone controlled by child.

the following: two of them consisted in both children playing with a videogame console, another design was a play tag game with drones, and the last one was a role-playing game without any kind of technology. Figure 10.5 presents a summary of these results.

\section{Observation vs. Participation}

Another aspect to consider was the degree of participation in the game of both players, the human and the animal. In three of the designs, the child was depicted just as an observer while the animal was playing. Those designs were: a child watching an eagle at the zoo, a child watching an elephant interact with a projected light and a child watching a snail on top of a remote control to see what it causes on a robot connected to it. Several designs showed a limited degree of interaction of the children, such as just feeding the animal (4), giving it vocal commands (1), watching through a camera the animal is carrying (1), and activating a technological artifact for the animal (2). The rest of the proposed games showed a high degree of participation from the children, especially in the designs involving technology, as the child was the one controlling the technological device used in the game as a mediator to interact with the animal (23). The remaining 20 designs from the NT participant group showed: the child using a non-technological artifact to interact with the animal (6), or both players performing the same activity 


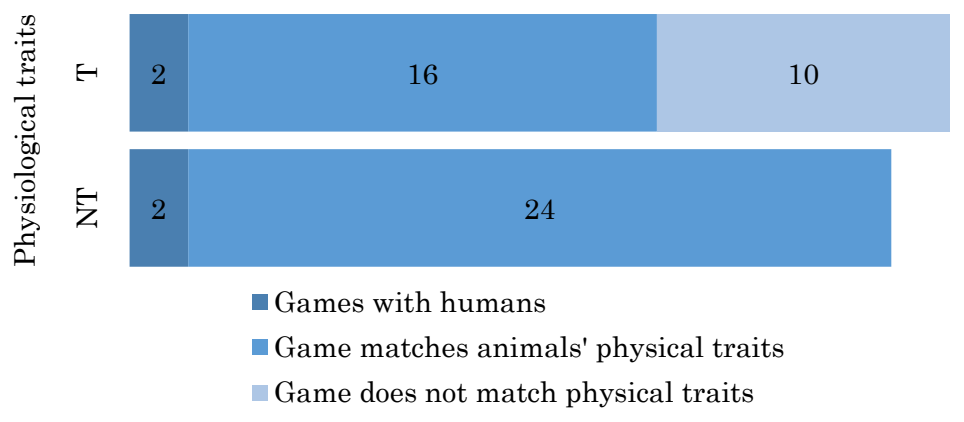

Figure 10.5: Matching of physical traits.

(14). Some examples of this latter case were racing to catch food with a gorilla or a crocodile, walking together with an elephant or a dog, playing hide-and-seek with an owl, untangling yarn balls with a cat, among others. Figure 10.6 outlines the detected degrees of participation.

Even though children were free to include as many animals and human players as they wanted, the activities they envisioned were usually for one animal and one human at a time. Only nine out of the total 54 designs included more than two participants, and in some cases they were only observers. For example, one child designed a game in which a fish had to catch an electronic robot in the water (see Figure 10.7a). She depicted herself with two of her cousins. Each of them had its own tablet and robot, and they were playing with several fish at the same time. Another child designed a game in which birds had to catch a ground robot he was controlling, but he started to add plenty of birds to the design, and eventually another robot so that they could all play, and even other animal species (see Figure 10.7c). The remaining designs included: two games from the same child in which there were relatives and friends just watching her play, a child playing with two friends, two games in which animals of different species were depicted but not participating in the game (see Figure 10.7b), and two games where animals of different species were depicted and interacted with the child. 


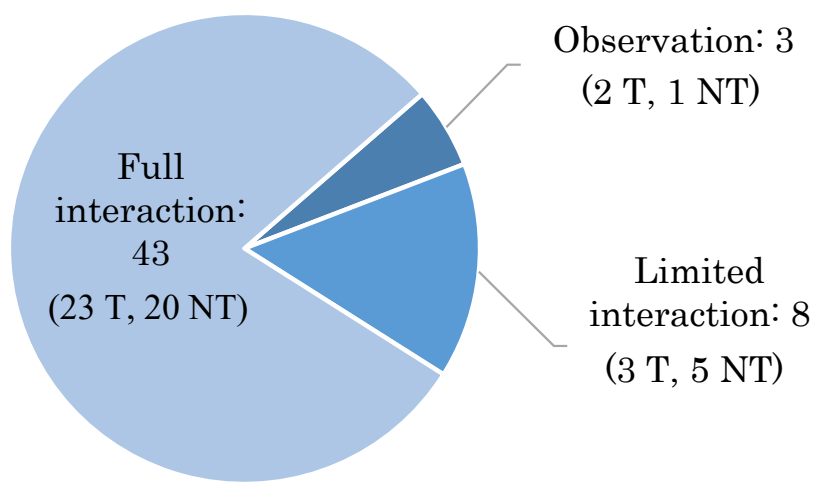

Figure 10.6: Degree of participation.
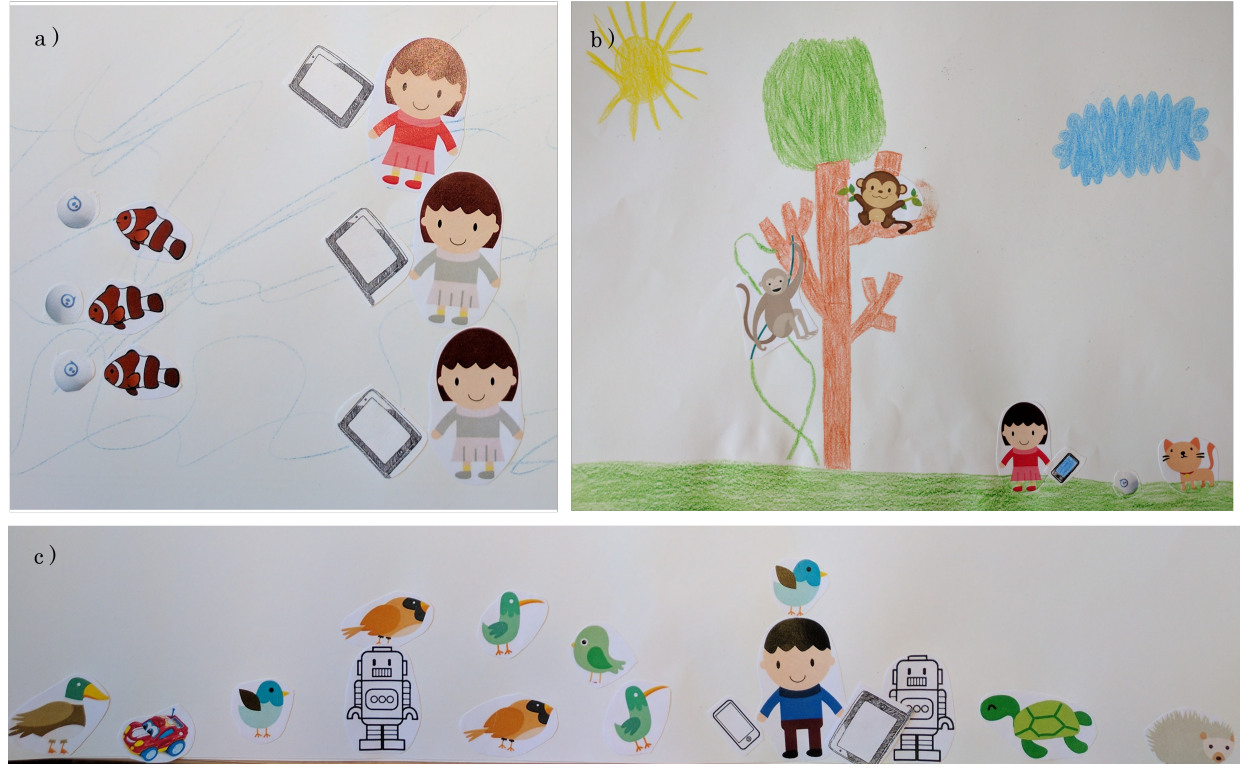

Figure 10.7: a) Three children playing with three fish, b) Animals observing, c) Several animals playing. 


\section{Anthropomorphism 8 Species Awareness}

A common situation that emerged in both $\mathrm{T}$ and $\mathrm{NT}$ participant groups was that the children tended to anthropomorphize the animal to some degree. The clearer examples of such case were that in four designs with wild animals (2 T, $2 \mathrm{NT})$ the children explicitly described the animal as a non-dangerous friend. They designed the game in such a way that the animal was capable of understanding the goal of the game and the child's intentions, and willingly play with the child (Figure 10.8a). Some designs were catching the ball with an orangutan or being thrown into the trees by an orangutan and swinging with him in the vines.

There were 31 games (17 NT, $14 \mathrm{~T})$ based on fostering animals' natural behaviors, exploiting the innate curiosity of the animal or replicating situations children have seen previously happen with that animal. Some designs involved wild animals following an object that carries meat, domestic pets catching an object or device, children feeding the animal or just observing the animal behave and move naturally.

The remaining 19 designs ( $7 \mathrm{NT}, 12 \mathrm{~T}$ ) showed games that require some understanding of the rules from the animal, or some kind of motivation so that the animal actually participates in the game in the friendly way the children described. For example, several children designed games for birds or fish outdoors that required the animal to catch an object, follow it or even play and return it to the child. This is something unusual, as these animals would probably run away from people. Other designs in this category include playing hide-and-seek with an owl, having a conversation with an elephant using a translation tool (Figure 10.8c), a race between a snake and a $\mathrm{RC}$ car, or a race between a crocodile and a human. Such designs assume that the animal understands what he is supposed to do and that it is interested in doing it. When children were asked about how they would get the animal to understand them, their answers were quite varied. Some answers were that the animal was indeed their friend, that they would first need to do some training with the animal using rewards, that the animal was already trained, and some of them said that they would explain the game to the animal although they did not know how.

The species and behaviors of domestic animals versus wild animals also played an important role in kind of game the children designed. For example, nine out of 13 children in the NT group designed games with different dynamics for the domestic and the wild animal they were given, e.g. playing fetch with a dog and a stick versus throwing food to an elephant in a zoo. Within the $\mathrm{T}$ participant group, only three children kept the same play dynamics for both animal species, e.g. bird or eagle that have to catch an electronic device. Ten children in this group changed the goal of the game depending on the animals' species, and two children in this group could only design a game for one species. 


\section{Animal-centric vs Human-centric Games}

Children were asked to design games they would like to play with a specific animal, and yet some of the games had a clear human-centric perspective. For example, one of the children designed a game in which a snail was to be placed on top or a remote control that activates a small moving robot. The child expressed he wanted to "see what it does", meaning that it would be fun to see what the movements of the snail cause on the robot. However, in this game the animal is not even aware of its participation in any kind of activity. This scenario of potential involuntary participation can be observed repeatedly among several designs. For example, one child draw a dog that was wearing a camera in its collar. This allowed the child to observe where the dog was, and what was it doing in case the dog escaped, helping him to find his pet. However, the dog was not aware of the camera nor participated in an explicit way. A girl described a game in which both a cat and herself had to untangle several balls of yarn, and the one who finished first won the game (Figure 10.8b). Despite that the game has been designed considering cat's innate behaviors, the goal of the game is human centric as it is unlikely that the cat understands the need of untangling the balls as fast as it can. Another example are three designs from different children that depicted a crocodile following a drone with meat, a tiger following a ground robot with meat, and a tiger following a drone. In all three cases, the children were controlling the devices but the animal did not seem to be aware that he was involved in any kind of activity. It was rather its predisposition towards eating or its curiosity that lead the animal to get involved in such game without being aware of it. Nevertheless, as reported in the previous subsection, several designs implied that the animal was a friendly being regardless of its species and behavior. Therefore, several designs that might initially seem to present an involuntary participation from the animal could be grounded on the idea of a common understanding of both players regarding the rules of the game. The postquestionnaire answers regarding the perceived animal's fun within the game will help to better identify how children understood the animals' participation in such designs.

\subsubsection{Questionnaire Results}

Children filled out a questionnaire before and after each design, whose contents are described in Table 10.2, Table 10.3 and Table 10.4. This section reports a summary of their answers.

Before each design, children were asked a few questions regarding their knowledge about a specific animal (PQ1 - PQ4). In addition, they were asked two 5point Likert scale questions (PQ5 and PQ6), which answers ranged from 1-Nothing to 5 - $A$ lot. When asked whether they believed the proposed animal would like to play $(\mathrm{PQ5})$, results were quite similar between domestic $(\mathrm{M}=3.83, \mathrm{SD}=1.20)$ and wild animals $(\mathrm{M}=3.62, \mathrm{SD}=1.20)$. Their answers were more dependent to 

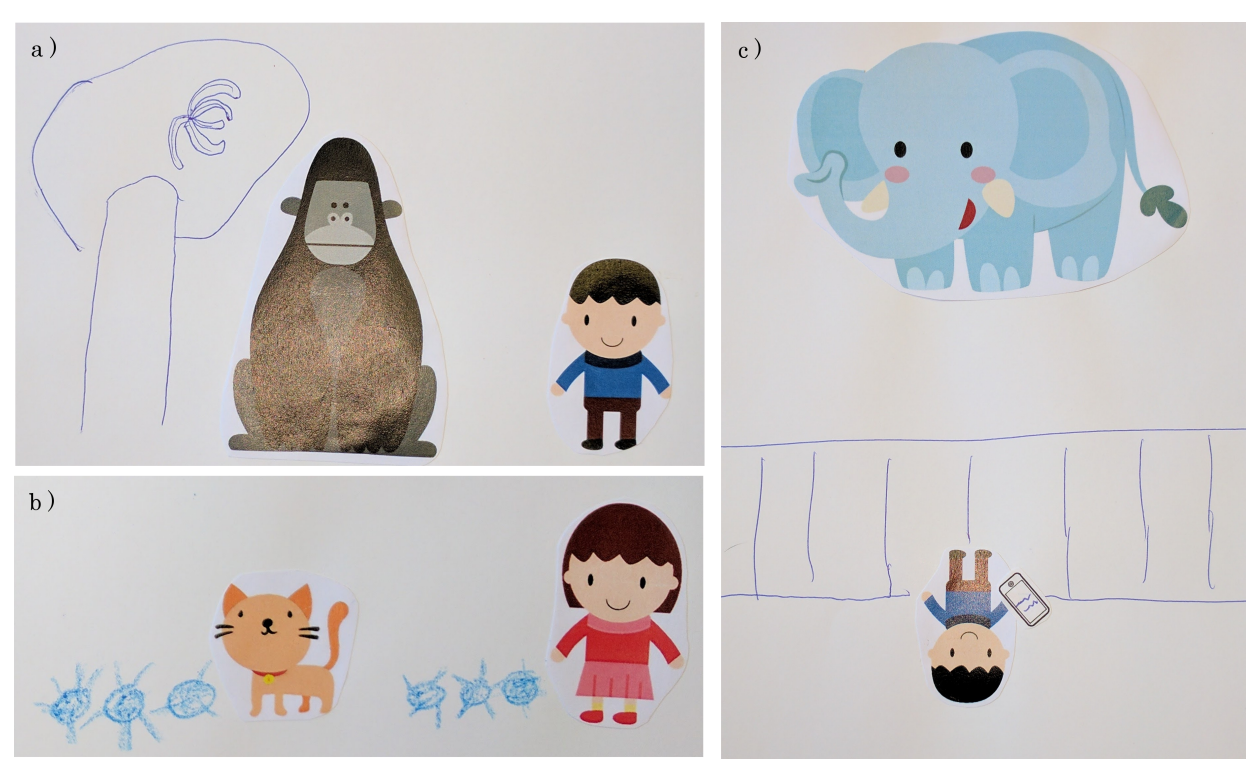

Figure 10.8: a) Child racing with a gorilla to get a banana, b) Child and cat playing to untangle yarn balls, c) Translation app to speak with an elephant.

the category of the animal: quadrupeds were considered more likely to play $(\mathrm{M}=$ $4.25, \mathrm{SD}=0.89)$, while reptiles $(\mathrm{M}=3.11, \mathrm{SD}=1.62)$ were considered less likely to participate in the game. Regarding whether they would like to play with the proposed animal (PQ6) children showed similar enthusiasm for both domestic (M $=3.83, \mathrm{SD}=1.40)$ and wild animals $(\mathrm{M}=3.50, \mathrm{SD}=1.68)$. Again, the category of the animal seemed to be influencing their results, being quadrupeds the most popular $(\mathrm{M}=4.75, \mathrm{SD}=0.46)$ and reptiles the least interesting ones $(\mathrm{M}=2.67$, $\mathrm{SD}=1.50)$.

After each design, the children were asked about the perceived fun of the human and the animal when playing the game they had described. The quantitative results for questions about human fun (TQ5) and animal fun (TQ6) for the two participant groups T and NT are reported in Figure 10.9. Overall, children considered that the games they designed were more fun for the human than for the animal. This could also be observed in the postquestionnaire interviews, in which several children expressed their concern about this matter. For example, participant P26 said that "perhaps the animal does not know that he is playing and might be scared, so I do not know if it would be having fun... ". Another participant, P1, also commented that she was not sure that the tiger would be having fun "because if he cannot understand me...", after what she suggested: "Perhaps if I give him his favorite food, when he learns what he has to do he would like it because he 


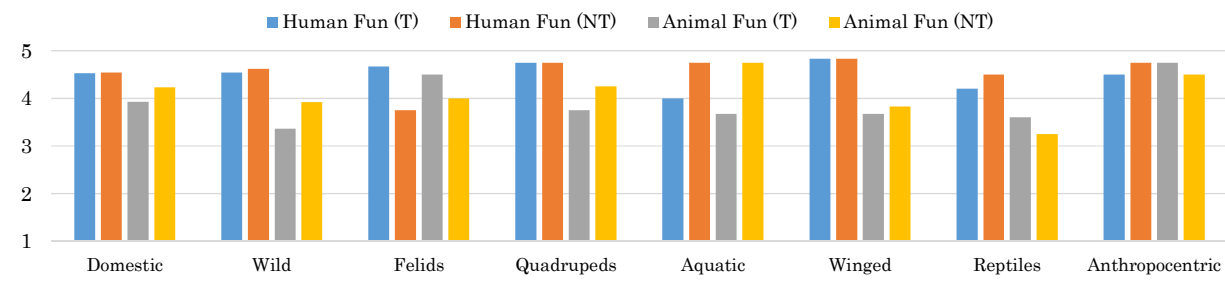

Figure 10.9: Postquestionnaire answers to perceived human and animal fun by category and type of animal.

would be eating, and tigers like eating". Overall, games designed for wild animals were on average reported as less fun than the ones for domestic animals in both groups of participants. One of the reasons could be the familiarity the children have with the species. In fact, designs for domestic animals were usually based on their natural behaviors or on replicating games that those animals usually play. Differences in animals' perceived fun could also be observed between species, e.g., the least fun games for the animal were perceived to be the ones for reptiles and birds. When asked about whether they would like to know more about the animal (TQ7), children's answer were quite positive. Most of the children answered that they would be quite interested in learning more things about domestic animals (M $=3.79, \mathrm{SD}=1.59)$, while for the wild animals they showed greater interest $(\mathrm{M}=$ $4.12, \mathrm{SD}=1.28)$.

Figure 10 shows the comparative results of the questions children answered before and after each design (SQ1-SQ5) in order to rate their perception about each animal in terms of how much they like the animal and how beautiful, friendly, intelligent and scary they considered it to be. Results show that children on average had a slightly more positive opinion about whether they like the animal after the design activity (SQ1), regardless of its species. They also showed a slightly more positive opinion about the animal's friendliness after the designs (SQ3), and they perceived the animal to be a bit less scary after the activity, especially in the case of wild animals (SQ5). The perceived intelligence of the animal (SQ4) was also higher for some species after the designs, as can be observed in Figure 10.

After the designs, children were encouraged to think about how they could make the game more fun for the animal, make the animal exercise more, make the game more easily understandable/usable for the animal, or make the animal learn something (TQ1-TQ4). Frequently, they were not sure on how to answer such questions, or after thinking a bit about it they just said the game was fine as it was. However, in several cases this exercise of reflection raised very interesting conversations. For example in TQ1, sometimes the children said they could 




Figure 10.10: Pre and post questionnaire answers to perceived aspects for the different animal categories.

improve the game to make it more fun but they were not able to explain how. Other times children described a new rule or feature of the game that would make it more challenging and therefore more fun to play, e.g. a faster robot, a robot that can go up the trees, involve another animal so that they have company. It is interesting that the most common answer was to add food or food rewards to keep the animal interested in the game over time or to make it want to participate in the activity.

Since many of the games involved the animal following an object, most of the answers to question TQ2 were that the animals were already doing enough physical exercise. In those cases in which the children believed that the animal could exercise more, some of their suggestions were to throw the object/device further away or move the device faster.

Thinking about making the game more easy to use or more understandable for the animal was definitely a challenge for the children (TQ3). Some of them realized during that part of the interview that perhaps the animal was unable to understand what it was supposed to do to participate in the game, and acknowledged that to the researcher. As has been discussed in the previous section, several designs fostered the animal's natural predatory/exploratory behaviors, such as following or catching an object. There were also designs based on what children are used to see when people interact with that specific animal species, e.g. dolphins in zoos or dogs playing fetch. In these designs, children's answers were usually that the animal would intuitively know what to do, even more if there was meat involved. In those designs in which the children considered that the animal would not easily understand what to do, they proposed several ideas. Some of them suggested to provide an example to the animal, such as another animal doing the same thing or a video of an animal performing the required behavior. Others explained they would train or teach the animal, usually using food-based rewards. Furthermore, 
some children described how they would use food to make the animal aware that it had to follow a specific path or object. A couple of children even suggested unrealistic scenarios such as "speaking the language of the animal" to explain the game to them. However, none of the proposed ideas was the redefinition of the goals of the game, changing the game dynamics nor the interactive artifacts. They instead looked for ways of making the animal adapt and understand the game.

Question TQ4 gathered children's opinion about whether the animals could learn something during the game (TQ4). This question was meant to assess whether the children conceived the game as a space in which the animal could learn new skills, or they instead tried actively to make the animal learn and respond to commands. This second case would also imply that the children consider the animal within the game as an entertainment for themselves and not as an equal and genuine participant. Results showed that children usually did not try to teach commands to the animals, which is a very positive outcome. Just in four designs, children reported that the animal could learn how to pay attention or learn new tricks. In some of the answers to TQ3 children proposed to train the animal to make it learn how to play the game, but they did not considered that process as the animal learning something new but rather as a tool to play the game afterwards. Answers to TQ4 showed that most of the children considered the game as a tool to help the animals enhance their skills, such as running faster, having more dexterity, being able to smell their prey and follow their tracks better, etc. Even two children commented that the game could help the animal to have a better behavior towards humans, such as not biting nor attacking people.

\subsection{Discussion}

\subsubsection{Improving Relationships}

The children joined the activity with interest and were overall very participative and motivated. Even though sometimes they expressed hesitation or surprise when the specific animal species for the design was revealed, they eventually came up with ideas and also expressed their doubts. Despite the fact that animals in the wild category were regarded as more scary or even some of them dangerous, children seemed to be more excited when designing games for them. However, games for wild species were also perceived by the children as less fun for the animal. The reason could be that it was more difficult for the children to envision a game for such animals, as they have not had any previous experience interacting closely with those species or they do not know any other behavior from the species rather than being fed or hunting their food. In fact, one of the things that stands out the most when analyzing the designs is the recurrent use of food and foodbased rewards for different purposes. Food has been used in the designs for just 
feeding the animal, but also for making the animal aware that there is some kind of interaction starting, draw its attention to some point or make the animal learn what he is supposed to do using a reward system. This was especially frequent in the case of wild animals. The reason might be that the activities in which children see some kind of interaction between a human and those animals are usually based on feeding the animal or making the animal perform a specific action they have been trained to. In order to help fill these gaps, more emphasis should be done on providing children with educational activities that focus on showing the animals' abilities and spontaneous behaviors without relying on food-based interactions. Zoos, in their role as educational spaces, could for example focus on enrichment activities for animals aimed at awakening the interest of the young public and increase their sensitivity and empathy towards the species [343] while learning. In this regard, technology could provide a powerful mechanism to create an immersive experience in which children could participate in that playful activity with the animal.

Another key aspect is that several children were capable of identifying from their designs that the animal might not understand the game, but were not able to adapt it properly. They rather tried to make the animal understand their supposed goal within the game. In other cases, some children might not have been aware of the implications the game had for the animal and designed human centric games. Given the motivation of children towards this kind of design activity, these could be redefined to present a guided design process that helps children reflect on their decisions and understand how to provide better interactions for the animals, without limiting the human participation. Based on the positive postquestionnaires opinions that showed an increased likeliness and perceived friendliness of the animals, these design activities could be a promising tool to help raise awareness and improve relationships in interspecies games.

\subsubsection{Collaboration}

Few designs showed collaboration between different children or animals at the same time. In fact, only a couple of designs depicted more than one human interacting with an animal within the game. Usually, the designs involved one child interacting with an animal at a time. The nature of the activity could be either observational, collaborative or competitive, e.g. observe/feed the animal, the animal follows an object controlled by the child, or the animal and child compete for the same goal. However, many of the designs could easily incorporate a social component for the children, such as rankings between friends based on how well they performed. Perhaps even games in which several children have to interact with different animals and work all together towards a same goal can be designed, fostering collaboration between humans and animals all together. The scores in these games could be based on goals that promote empathy and an 
ethical interaction with the animal, encouraging children to create a strong bond with them while learning and enjoying.

\subsubsection{Remote Interaction}

The children always depicted themselves in the same physical location as the animal. None of them designed a scenario in which the children was in a different place than the animal. Perhaps this is a more complicated scenario for them to envision, as they are not aware of the technological possibilities. Currently, children can observe animals in prerecorded videos, TV documentaries, live streams, or even using remotely controlled cameras [84]. However, they have not interacted before with an animal that is located far away from them using a technological device. This kind of remote interactions, if carefully designed, could allow the children to connect with their pets at home, or even with species that are not commonly accessible, such as wild animals in zoos. This would be a powerful and motivational tool to learn about many different species in an immersive experience. In addition, this kind of remote interactions for hospitalized children can provide positive effects for the children's wellbeing during their stay. These designs must not forget about the animal's requirements, and could for example provide clues to the children on what to do during the game so that they can learn along the way in which ways the interaction is beneficial for the animal, what the animal wants and how should we interact with them to be respectful.

\subsubsection{Threats to Validity}

There are several aspects in this study that may have influenced the results. First, the number of participants that designed games for the same category of animals is relatively low. It was sufficient for the purpose of this study in order to gather observational findings. However, a greater number of subjects is required to conduct a more detailed statistical analysis of the results. Second, given the particularity of the environment in which the experiment was carried out, children's mood or behavior might differ from other environments such as home or school. Nevertheless, children were observed to enjoy the activity, they were not long-term hospitalized children and the pediatric school is meant to be a welcoming and friendly space for them. Finally, the thematic analysis conducted over the results was performed as objectively as possible. However, due to the researchers' background in HCI and ACI, perhaps some categories have been underrepresented or could have another interpretation. 


\subsection{Conclusion and Future Work}

This paper has presented a study in which children designed interspecies games between humans and animals with and without technology. Results showed that children found easier to design games for domestic animals, by reflecting on common activities with such animals due to their familiarity. On the contrary, games for wild animals were harder to envision. Although many of those games were based on fostering the animals' natural behaviors, children frequently used food as a mechanism to encourage the animal to play. This exposes the need for developing educational activities oriented at children that help them bond with non-domestic animals and discover their natural behaviors without relying on food-based interactions. The design process also seemed to positively affect children's opinions about animals, improving the perceived friendliness, intelligence, scariness and likeability of the animals after the activity. It also helped children to reflect on the suitability of their design for the animal. While some of them understood their design was human-centric, few were able to change it to a more animal-centric one. Therefore, this kind of design activities reveal as a promising tool to encourage critical thinking, and we aim to redesign the process in order to guide children towards a better understanding of animals as co-players, which would help to greatly improve their relationships with animals.

In addition, the game dynamics observed in the designs will help to develop interspecies technological games for children and animals, with the added benefit of having both children and animals as co-designers of the game. These games will be used by hospitalized children so that they can remotely interact with animals in zoos, shelters or daycare facilities. Therefore, these systems will also provide a suitable interactive activity for an animal who might also require further stimulation. The game could also present children with hints or information about the animal, and the most suitable activity or way of interaction with them by detecting the animals' behaviors and actions during the game [255, 257]. Such playful interactions could improve both animals and humans' wellbeing in their respective contexts, while creating strong bonds between species.

\section{Acknowledgments}

This work is funded by the European Development Regional Fund (EDRF-FEDER) and supported by Spanish MINECO (TIN2014-60077-R). The work of Patricia Pons is supported by a national grant from the Spanish MECD (FPU13/03831). Special thanks to the children who participated in our study, and the teachers from Unidad Pedagógica Hospitalaria La Fe for their valuable collaboration and dedication. 


\title{
Chapter 11
}

\section{Remote Interspecies}

Interactions: Improving

humans and animals'

wellbeing through mobile

playful spaces

Patricia Pons ${ }^{1}$, Alicia Carrion ${ }^{2}$, Javier Jaen ${ }^{1}$

\footnotetext{
${ }^{1}$ ISSI Group, Departamento de Sistemas Informáticos y Computación, Universitat Politècnica de València, Valencia, Spain

${ }^{2}$ Departamento de Personalidad, Evaluación y Tratamiento Psicológico. Facultad de Psicología, Universitat de València, Valencia, Spain
}

Submitted to Pervasive and Mobile Computing. Under review, as of June 2018.

\begin{abstract}
Play is an essential activity for both humans and animals as it provides stimulation and favors cognitive, physical and social development. This paper proposes a pervasive playful environment that allows hospitalized children to participate in remote interspecies play with dogs in a canine daycare facility, while it also allows the dogs to play by themselves with the pervasive system. The aim of this playful interactive space is to help improving both children's and animal's wellbeing and
\end{abstract}


their relationships by means of technologically mediated play, while creating a solid knowledge base to define the future of pervasive interactive environments for animals.

\subsection{Introduction}

Play stands as one of the most natural and inherent behaviors among human and non-human animals. As Huizinga described in his Homo Ludens, "Play is older than culture, for culture, however inadequately defined, always presupposes human society, and animals have not waited for man to teach them their playing" [131]. The nature and importance of play have been widely studied and emphasized over the years. One of the main aspects of play is that it is fun, and this is the main source of motivation for all sorts of animals, including humans [108].

In our digital society, we have evolved play, making it even more appealing with the development of technological innovations that allow us to enhance our playful interactions with newer and more varied experiences. From the very first arcade videogames and video consoles with traditional joysticks and gamepads, to the latest playful environments based on Natural User Interfaces (NUIs) [8, 27, 127], the spaces in which playful interactions take place are becoming more and more ubiquitous $[100,166,306]$. What remains constant in all these innovations around play is that although its inherent nature may have several purposes that are not yet completely understood [19], it remains essential for cognitive, physical and social development [93], especially in the case of children [93].

Nevertheless, humans are not the only species using technology to improve their playful experiences: non-human animals are also experiencing a digital revolution with emerging research around technology to support animal play [75, 247, 260, 343, 349, 361], in what has been coined as the Animal Ludens revolution [254, 258]. Over the past years, the field of Animal Computer Interaction (ACI) [173, $174,178]$ has gained a lot of attention, proposing the design and development of technology for animal use following a user-centered approach. The main goal of ACI research is to improve the animals' wellbeing by designing suitable technology for them. Playful interactions of animals with technology have been extensively studied [142, 258, 351, 363], as they can help to improve the animals' wellbeing, reduce stress, and provide the animal with physical and mental stimulation [258, $363]$.

Technologically mediated play can therefore provide many benefits for both children and animals, such as alleviating stress and anxiety. This is especially relevant when these individuals are exposed to high loads of emotional stress and insecurity. On one hand, this is the case of hospitalized children, who can experience social and emotional issues due to the hospitalization experience and the illness 
itself. On the other hand, there are millions of domestic pets and wild animals in zoos or sanctuaries who have to spend long periods alone, which might cause them boredom, distress and feelings of isolation. Technology has been used separately in both domains to provide children and animals with mental and physical stimulation to help alleviate these problems. However, technology could also be used to support children playing together with animals. In this case, the benefits of playful technology would be added to the positive effects the relationship between animals and patients may have on both actors [12, 13, 136, 215]. This would add a completely new range of possibilities to help improve both children's and animals' wellbeing. The only major drawback is that not all hospitalized children can receive the visit of therapy animals or companion animals, due to their clinical condition. Hence, we believe it is essential that technology could allow for remote playful interactions between humans and animals, especially in the case of humans and animals who are unable to spend time together or cannot move from their physical location.

This paper proposes a pervasive remote interactive system that allows hospitalized children to play with the dogs in a canine daycare facility by remotely controlling a small robot located in the facility with the dogs, as well as dogs playing by themselves with the system. The proposed playful interspecies system aims to (1) help improve the children's wellbeing during their hospitalization, (2) foster a healthy relationship between children and animals by means of play, and (3) provide physical and mental stimulation for animals at home, in shelters or in daycare facilities. The outcomes of this work are manifold. First, to the best of our knowledge, this is the first remotely controlled system that allows children to play with animals physically separated from them. Secondly, the effects of these playful interactions have been evaluated for both children and animals, allowing us to detect usability issues for both species, and to assess the impact of the interaction on children's perception of animals. Finally, a comparison was made between the child-controlled robot system and a pervasive system that autonomously plays with the dog. This allowed the definition of future steps and recommendations for the development of intelligent playful environments for animals capable of providing an engaging and meaningful experience for the animal, advancing research within the ACI field.

\subsection{Related Works}

This section contains a review of the current literature regarding interactive playful systems for hospitalized children and animals, highlighting the importance of creating technology to support and foster human-animal relationships using pervasive scenarios that allow for natural interactions for both animals and humans. 


\subsubsection{Applications for Hospitalized Children}

There have been many studies in the literature in which the use of game technology has helped reduce the negative impact of hospitalization on children and improved their wellbeing inside the hospital [144]. While most of these works use traditional computers and monitors, handheld devices are gaining attention [144]. These devices are more suitable for children with physical discomfort/impediments as they are less physically demanding. In addition, mobile devices allow to create more ubiquitous applications which children could use in their own hospital room.

Several works have studied the combination of animal therapy and games in hospitalized patients, observing their positive effects on morale and stress reduction in kids [145]. However, introducing live animals into a hospital is not always possible, and different approaches are needed to provide similar effects on the patients' wellbeing. Several works have replaced real animals with robotic ones, such as a rabbit [25, 26], a parrot [299], a teddy bear [135], a mammoth [97, 98], or cats and dogs [150]. Other studies involve real animals that do not necessarily have to be present at the hospital. Zootopia [1,2] is a tangible-based game that allows children to explore live videos of animals. It consists of a board representing several ecosystems with their respective animals. When the child places a toy with an RFID tag in front of an animal on the board, a live video stream is played showing this animal in a real zoo. However, the camera cannot be controlled, which results in many empty-screen moments. HabitApp [84] proposes a tablet-based application that allows children to control the cameras located in a zoo environment to actively observe animals at the children's will. Although these works allow for exploration and observation of real ecosystems outside the hospital in real time, none of them permits direct interaction with the animals or their ecosystem. Being able to remotely play with the animals in real time would add a very powerful new dimension to these activities with regard to improving the children's motivation during their hospital stay.

\subsubsection{Playful Applications for and with Animals}

Playful interactions of animals with technology have been one of the main lines of research within ACI $[254,362]$. These technologically-mediated playful experiences for animals usually involve a human participant who either controls the activity or plays together with the animal.

Felino [351] and Cat Cat Revolution [224] proposed a tablet-based game for cats in which the animal has to catch some creatures that appear on the screen. The human can adapt the elements that appear in the game to the cat's preferences [351] or move them around to play with the animal [224]. A similar approach is the use of tablet-based applications for zoo enrichment. The Apps for Apes [10] project consists of a set of applications that orangutans can play with on 
a tablet. However, a zookeeper outside the enclosure has to hold the tablet for the orangutan and the interaction is therefore quite limited. The Kinecting with Orangutans [343] project goes one step further and allows the orangutans to play with elements projected onto the ground of their enclosure, using projections and depth sensors, thus allowing the animal to interact more naturally. Non-wearable tracking systems have been proven very useful to allow natural interactions of the animals within a more pervasive game scenario [247, 257, 343]. Purrfect crime [323] also proposed the use of depth sensors and projections to create digital games for animals and humans. In this game, both the animal and the human have to catch digital birds projected onto the ground. The human uses a remote control to point and drag the birds, and the cat can move freely around the space while the depth sensor captures its position.

Most of these works require the human and the animal to be in the same physical space. One of the very few works that allows a human to play remotely with an animal is the Playing with Pigs project [5]. In this project, the human uses a tablet application to move a visual digital element that appears on a wall-screen in a pig enclosure. The goal is that the pig touches the digital elements with its snout and reaches a desired target area. The human does not need to be physically present with the pigs to play with them. However, although this was a novel idea, it has not been developed further. Another example is LonelyDog@Home [129], which allowed a human to connect over the internet to a web camera and remotely feed her dog, as well as throw a ball to the dog. Although it is one of the first works within ACI and allows remote interaction, the playful activity is quite limited, as the human can only throw the ball towards the same place all the time.

There is thus a clear need to evolve interactive spaces for animals and humans towards a more pervasive solution $[254,257]$ in which the animal can move freely around the space and the playful interactions can be more varied. In addition, some animal species such as dogs or cats might prefer tangible devices to play with $[260,349]$. As the current digital experiences do not offer these affordances, the use of tangible devices for animals within these playful interactive systems would be a promising opportunity.

\subsubsection{Designing to Improve Relationships between Humans and Animals}

ACI research has recently begun to explore how technology could help to improve the relationships between humans and animals [248, 326, 343, 351]. Several works have studied the perceptions of humans towards animals' interactions with technology in different contexts. For instance, discovering how pet owners perceive their dogs' reactions towards technological devices can help to raise awareness of good or bad practices in digital interactions of animals with technology $[18,117]$. On the other hand, animal conservation organizations aim to provide empathic 
responses of visitors in order to foster interest in wildlife, and technological installations have shown to be promising in this regard [111, 343].

The design of playful technology for animals with an animal-centered design perspective has also allowed humans to empathize more with the animal species, helping to create stronger concern and bonds. This has been shown not only when the designers of the playful scenario are experienced researchers $[142,349,353$, 361 , but also when the human has no previous design experience at all, such as children [248].

Humans have described these playful activities with animals, from their design $[142,248]$ to their realization $[224,343]$, as "useful spaces to reinforce their relationships and to create new forms of communication with the animal" [224]. As the world is becoming more interconnected, these communicative spaces do not need to happen in the same physical space. By expanding the horizon of playful shared spaces towards remote and pervasive scenarios, the benefits of such playful experiences could be extended to other populations that have physical constraints. In the case of playful experiences for humans and animals, remote scenarios would allow them to experience a bonding activity without the limitation of being together in the same place.

\subsection{Technological Platform}

This work proposes the design, development and evaluation of a remote playful system in which children can control a tangible robot to play with an animal in a different location. Additionally, on the animal side of the interaction, a pervasive system has also been developed capable of controlling the robot autonomously to play with the dog without human intervention.

The selected technologies for both the human and the animal in the proposed remote playful game should be aimed at facilitating the interaction by adapting to the user's physical and cognitive characteristics. In the case of children, previous work by the authors showed that within a co-design activity children envisioned technologically mediated games with animals as a playful scenario in which the child was in control of a robotic element the animal could play with [248]. The children's user interface will therefore consist of a mobile application they could use even from their own bed at the hospital. In the case of animals, previous work has shown that purely digital stimuli might not have all the effect that tangible elements can provide for some species [252, 260, 349], such as grabbing, touching, biting, etc. In this regard, our proposed system will use a tangible robotic ball to interact with the animal. In addition, for the non-human mediated game between the system and the dog, a non-wearable tracking system would allow the animal to 
behave more freely and with more spontaneity [255, 257]. For this, a non-wearable tracking system capable of detecting the dog's movements has been implemented.

The playful remote system proposed and evaluated in this paper consists of two separate applications that communicate remotely over the network (see Figure 11.1). We deployed a Sphero ${ }^{\circledR}$ robot and a Microsoft Kinect ${ }^{\circledR}$ v2 sensor in the canine daycare facility to record the play area and track the dogs' movements. A .NET streaming server was developed to record images from the sensor using the Microsoft Kinect ${ }^{\circledR}$ SDK 2.0 and C\#. A Universal Windows Platform application was also developed using the Sphero ${ }^{\circledR}$ SDK for UWP, which allows any available Sphero ${ }^{\circledR}$ robots around to be discovered, connected and sent basic commands via Bluetooth. The Sphero ${ }^{\circledR}$ UWP controlling application exposes a UWP AppService on the computer on which it runs, with which the .NET streaming server can communicate with to request its services when running on the same machine.

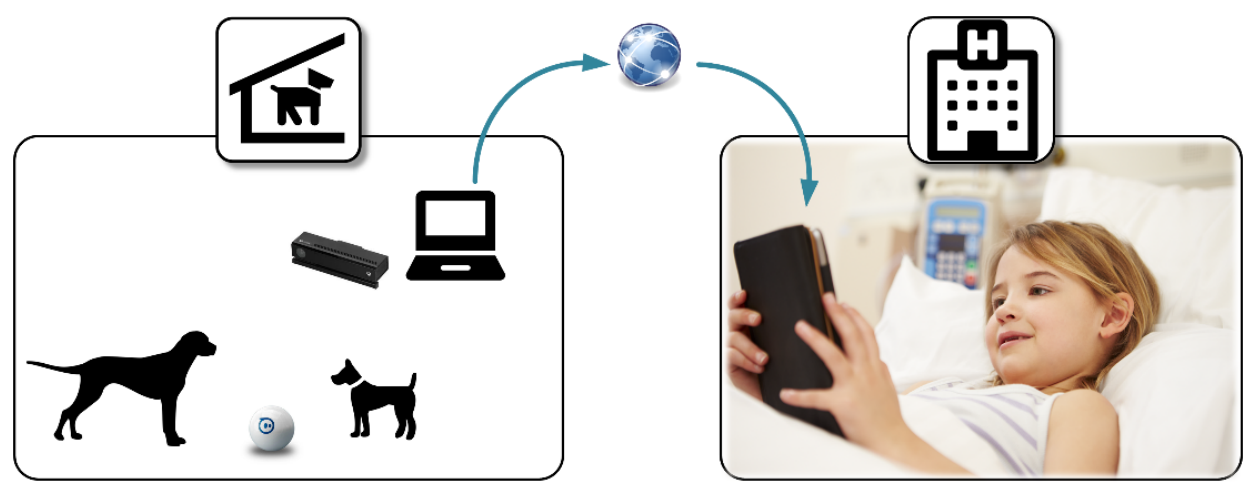

Figure 11.1: System deployment and set-up.

On the client side, we developed an Android application that connects to the streaming server from any location on the network, receiving and displaying real time images from the daycare facility on the screen of the mobile device. This Android application is installed on a mobile phone and used by children at the hospital. Once the client application connects to the server and starts receiving images, it displays them on the screen along with four buttons to control the Sphero ${ }^{\circledR}$ robot (see Figure 11.2). When the user presses a button on the Android application connected to the server, the corresponding command is sent to the server at the canine facility in real time. The server then transfers the command to the Sphero ${ }^{\circledR}$ AppService requesting it to move the robot with the selected command. The Sphero ${ }^{\circledR}$ UWP application then communicates with the robot via Bluetooth and sends the moving command to it (see Figure 11.3). The experienced average delay of the system is $280 \mathrm{~ms}(\sigma=132.5 \mathrm{~ms})$. 


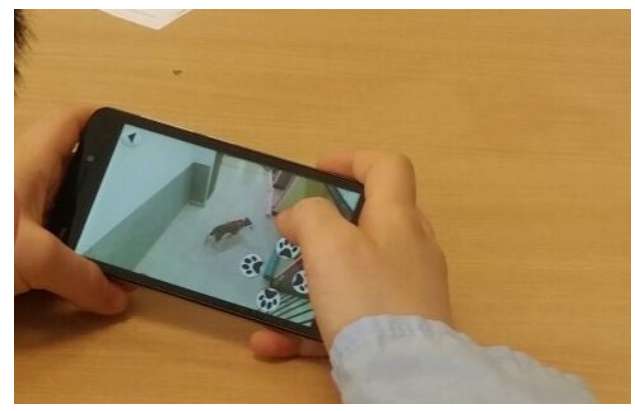

Figure 11.2: Hospitalized child controlling a Sphero ${ }^{\circledR}$ to remotely play with a dog in a canine daycare facility.

\subsection{Observational Study on Children}

This section describes the observational study conducted on hospitalized children using the remote interactive application to play with a dog. The aim was to evaluate the playful experience of children in terms of usability, enjoyment and effectivity in terms of improving the animal-human bond.

\subsubsection{Participants}

The participants were hospitalized children from the Hospital Universitari $i$ Politècnic La Fe in Valencia, Spain. The hospital has its own school in the pediatric wing to which children from primary and secondary school can attend, although the pediatric oncology patients usually need to stay within a designated area. The sessions of this study were therefore conducted either in the pediatric school room or in the child's own room at the hospital. Twenty-one children participated in the study (11 girls and 10 boys, 15 children from pediatric school and 6 children from pediatric oncology), with ages from 4 to 15 years old $(\bar{x}=9.43, \sigma=2.66)$.

\subsubsection{Methodology and Procedure}

Each child performed the activity individually with the researcher. The sessions lasted approximately 10-15 minutes, according to the child's interest in the system and the time needed to answer the postquestionnaire.

At the start of the session, the researcher briefly introduced the activity to the child, explaining that she was going to control a robot using a mobile application in order to play with a real dog located in a canine daycare facility nearby. Once the researcher had introduced the activity, the child received the mobile phone 


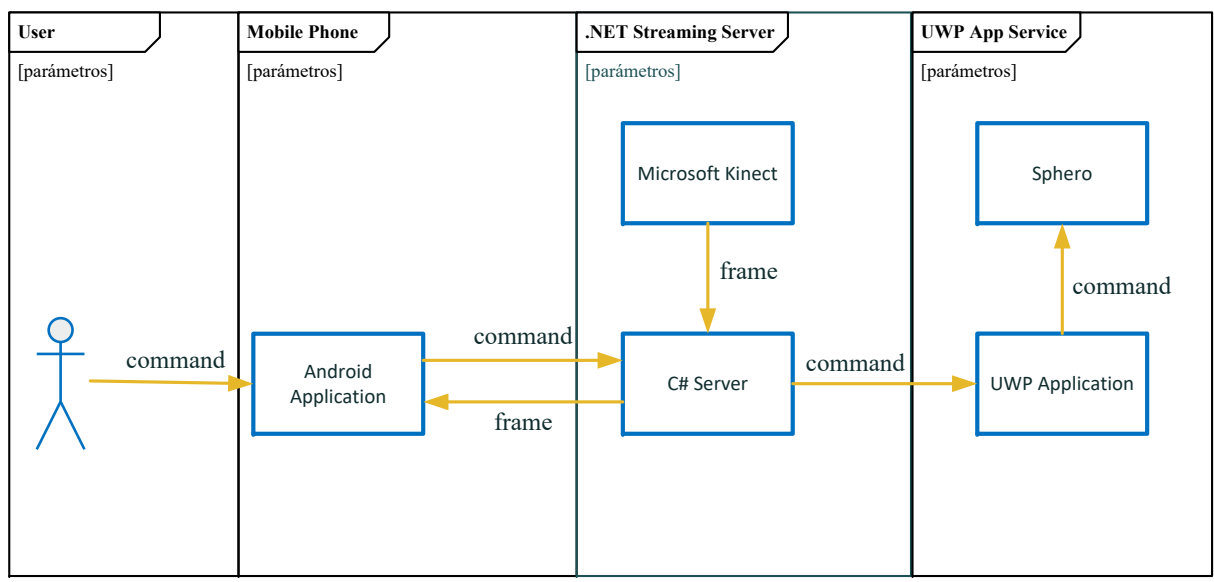

Figure 11.3: Communication between applications.

with the application already connected to the server and streaming images from the canine facility. Each child was allowed to use the application for 5-10 minutes, with no initial training, and was then encouraged to explore what happened when she pressed the buttons displayed on screen. While the child was using the mobile application, the researcher filled in an observational template, also noting observations and any verbal feedback the child might give.

After the game, the children filled in a brief postquestionnaire about the animal and the activity, consisting of 5-point Likert questions based on the Fun Toolkit questionnaire [266] (Figure 11.4). These questions identified usability issues, the children's perceptions regarding the animal, assessing whether they enjoyed the game and if they thought the game would be a positive experience for the animal as well. Table 11.1 shows a summary of the questions the children answered after the activity.



Bad / Nothing

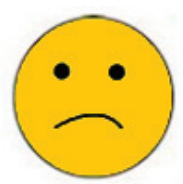

Not great / A little

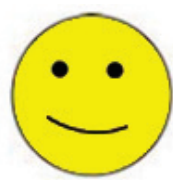

Good / Neither much nor little



Very good / Quite

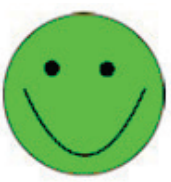

Great / A lot

Figure 11.4: Example of the 5-point Likert scale answer options. 
Table 11.1: Postquestionnaire 5-point Likert questions and open answer question.

\begin{tabular}{cc}
\hline Postquestionnaire & Code \\
\hline How much did you enjoy playing with the animal? & Q1 \\
Would you like to play with the animal again? & Q2 \\
Would you like to play this game with other children? & Q3 \\
Do you think the animal enjoyed playing the game with you? & Q4 \\
Do you think playing this game is good for the animal? & Q5 \\
Would you like to know more about the animal? & Q6 \\
Would you have liked to have more animals playing together? & Q7 \\
How much do you like this animal? & Q8 \\
Do you think it is friendly? & Q9 \\
How easy was it to control the robot? & Q10 \\
What would you change in the game to enjoy it more? & Q11 \\
\end{tabular}

\subsubsection{Observational Results}

One of the first observations of most of the children was their positive surprise when they saw the animal moving and reacting to the robot's movements they were performing. Even after the activity had been explained to them, describing that the dogs were actually in the canine facility so that they could play with them, some children asked again to confirm whether the video was being received in real time. One of the children's most frequent questions was the name of the dogs they were playing with, and a few of them asked questions such as "What is the dog doing there?" or "Are there more dogs around?".

Overall, the children easily learned how to control the robot with precision. Only three children were observed to have issues with the application. One of these was four years old and was the youngest in the study, another had a restricting physical condition and required an assistant to hold the mobile phone for her, while the last reported she did not like dogs and showed little interest in the activity in general. Few issues were reported by the children during the activity, and these mostly involved difficulties in controlling the robot when it went out of the camera's field of view. Only one child complained about the delay in the image and another reported that the robot moved too slowly.

Only five children were observed to be frustrated or expressed a certain degree of annoyance when the dogs did not react as they were expecting. In spite of this, they kept trying to move the robot to capture the attention of the dog, or stopped playing with the robot and just observed the dogs move around. Most of the children did not complain about the dogs' behavior and even when the dogs 
did not play with the robot the children were keen to keep trying new movements to see whether they could make the dog play.

Almost all the children showed signs of joy and amusement from the beginning of the activity: smiling, laughing, commenting aloud what the dogs were doing, calling the animals by their names and talking to them as if they were in the same space, etc. In a few cases the children were more neutral in their reactions: one was not interested in the activity, another was highly concentrated, another did not seem excited as she was playing with a dog that was not very playful, and in one case the child was experiencing physical discomfort due to her condition.

The researcher rated each child's perceived interest in the activity on a 5-point scale. Overall interest in the activity was really high, with an average of 4.5 points $(\sigma=0.75)$. These results can be compared to children's answers to Q1 in the postquestionnaire (Section 11.4.4, Table 11.2), showing that they were highly motivated towards the activity and enjoyed the experience.

\subsubsection{Questionnaire Results}

After playing remotely with the dog using the Android application to control the Sphero robot, the children were asked to fill in a brief postquestionnaire containing eleven Likert scale questions and one open answer question. Figure 11.5 shows the results of the Likert scale questions listed in Table 11.1, while Table 11.2 contains the mode, mean and standard deviation for each question. It can be observed that more than $50 \%$ of the answers to all the questions were rated with the highest scores (4 or 5$)$, with low standard deviations ( $<1.5$ points).

Table 11.2: Mode, mean and standard deviation of children's answers in the postquestionnaire.

\begin{tabular}{cccccccccccc}
\hline & Q1 & Q2 & Q3 & Q4 & Q5 & Q6 & Q7 & Q8 & Q9 & Q10 & Q11 \\
\hline Mode & 5 & 4 & 5 & 5 & 5 & 5 & 5 & 5 & 5 & 5 & 4 \\
Mean & 4.24 & 4.05 & 3.67 & 3.57 & 4.19 & 4.38 & 4.48 & 4.48 & 4.48 & 3.95 & 3.33 \\
$\sigma$ & 0.77 & 1.16 & 1.43 & 1.29 & 0.81 & 1.02 & 0.98 & 1.08 & 1.03 & 1.40 & 1.39 \\
\hline
\end{tabular}

The first three questions were intended to assess the children's overall enjoyment of the activity. In Q1, they were asked to rate their enjoyment in playing with the animal with the mobile application. $80.96 \%$ showed great enthusiasm for the activity and rated their enjoyment very positively, with either four (Very good) or five (Great) points. In fact, none of the children rated their enjoyment with one (Bad) or two (Not great) points, even when the child was seen to be distracted or not very interested in the activity. When they were asked whether they would like to play with the dog again using the remote mobile application (Q2), their answers were even more positive than in Q1. Most were willing to repeat the 


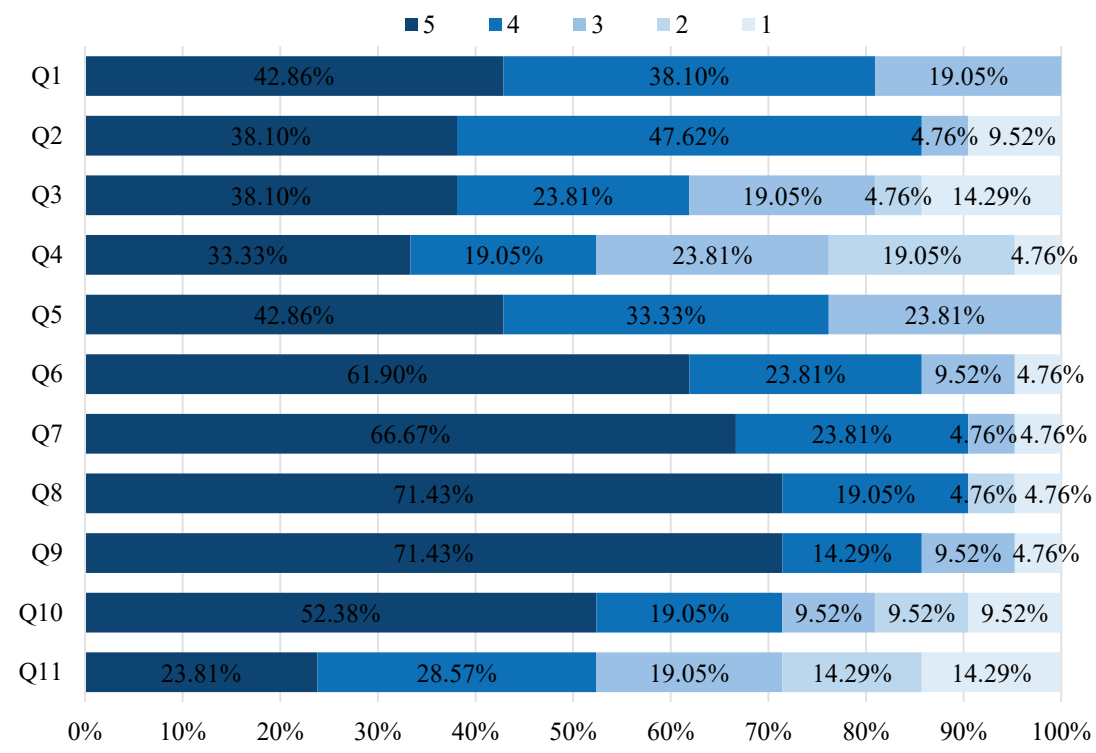

Figure 11.5: Postquestionnaire results from the Likert scale questions.

playful activity, with $85.72 \%$ of the children reporting that they would really like to play with the dog again, with scores of five (A lot) and four (Quite). Only two said they would not like to repeat the experience. One of these children reported lower levels of enjoyment in Q1 than the rest of the participants, and showed poor interest in the activity. The other participant who was not willing to play again was very young - 4 years old - and although she showed great interest in the activity, the researcher reported some difficulties in the interaction. Q3 was meant to assess the collaborative aspect of the activity, asking the children whether they would like to play this interspecies game with other children. Socialization is a very important factor in hospitalized children, and mobile environments are ideal for having shared spaces in which they can create a positive hospital experience and reduce their sense of isolation [159]. In this regard, $61.91 \%$ of the children said that they would like to share this experience with other children. Only three $(14.29 \%)$ reported they would prefer not to play with other children. One of these had reported she did not want to play the game again, and the researcher also noted she did not seem interested in the activity. However, the remaining two children expressed a lot of excitement about playing with the dogs again, and from their results it could be argued that they preferred to have the activity just for themselves.

A recent trend in the field of Animal Computer Interaction aims to investigate how technology can help improve the relationships between animals and humans 
$[142,248,326,349]$. Previous research has explored how children perceive animals before and after designing playful interspecies environments with them [248], revealing that this exercise of co-design positively affects the children's perception of the animal. Questions Q4 to Q10 were therefore aimed at assessing whether they considered the game to be a positive experience for the animal and how the playful interactions with dogs affected their relationship with and perception of the animals.

The children were asked whether they considered the animal enjoyed playing with the robot (Q4), with $52.38 \%$ rating the animals' enjoyment with five (A lot) and four (Quite) points. This was one of the questions with the highest variability in the answers $(\sigma=1.43)$, and could be explained by the dog's behavior in each case. If the children saw the dog was not interested in the game, without directly interacting with the robot or looking in the opposite direction, they were more likely to evaluate this response as low enjoyment. Some children gave as reason for their answer that "the dog was not paying attention to the robotic ball" or that "it depends on the personality of the dog, some of them might like to play with this ball while others not", showing their awareness of the animal's preferences and needs. It is interesting to note that, although some children perceived that the animal they were playing with did not seem to be interested in the activity, $76.19 \%$ believed the activity was beneficial for the animal (Q5). This shows clear distinction in the children's perceptions between what the animal might need due to its personality and the idea that animals need exercise and fun in order to be healthy and to provide them with physical and mental stimulation. None of the children considered this activity bad for the animal in any degree. The participants showed a lot of interest in knowing more about the animals they were playing with (Q6), with $85.71 \%$ rating this question with four or five points. The children said they would like to know if the animal liked to play, what type of character and personality it had, e.g. if it behaved well in its daily routine, and what does it like to do and play with when it is not playing with the robot. The children reported whether they would like to have more animals playing together with the robot at the same time (Q7). In contrast to the results of Q3, on sharing this activity with more children, they preferred to have more animals playing, with $90.48 \%$ of the answers rated with 4 or 5 points.

Questions Q8 to Q10 focused on the children's perception of the animal in terms of likeability, friendliness and intelligence, respectively. Overall, most of the children liked the dog from "quite" (19.05\%) to "a lot" (71.43\%), and considered the dog to be quite $(14.29 \%)$ or very friendly $(71.43 \%)$. Their perceptions of the dogs' intelligence were a bit lower than their friendliness, with $52.38 \%$ of children reporting the animal to be very intelligent or at least quite intelligent $(19.05 \%)$. These latter responses could be explained by the animals' interest and engagement in the activity: if the dog showed poor interest in the robot or did not want to chase it, perhaps the child considered this animal less intelligent because it did not 
pay attention to the robot or reacted as expected. The child that reported she did not like dogs also considered the dog to be unfriendly and not at all intelligent, being the only participant whom the observer reported to have little interest in the activity as she was distracted by the things happening around her.

The last Likert scale question (Q11) addressed the application's usability issues, asking about the perceived difficulty of moving the robot to the desired place. Overall, $71.43 \%$ of the children reported that controlling the robot was either easy, very easy or "super" easy (rating this question from 3 to 5 points). Of the children who considered it difficult or somewhat difficult, one reported the delay in the response as the main issue, and two explained that the robot had got stuck where they could not see it, e.g. in a corner, and could not easily return it to the visible area. Another child reported the interaction as difficult because he considered the robot was slow and he wanted it to move faster. No perceivable difficulty in the interaction was observed by the researcher in the remaining cases, and these children did not give the reasons for their answer either.

Lastly, the questionnaire gave the children an open-answer question (Q12) about what they considered important to include in the game to make it more fun and engaging, either for themselves or for the animals. Some of the suggestions were to have light, sounds and even smells on the robot to make it more interesting for the animal. Another suggestion was that they would like to hear the animals in addition to seeing them on the screen. Several children said they would love to see other animals in the daycare facility, not just the ones they are playing with, as well as having a wider camera angle or even the camera installed inside the robotic ball to see the dogs better. A couple of children suggested feeding the animal by either using the robotic ball as a challenge to get a food reward, or by being able to move the food around and give it to the animal. Some of them wanted the animal to be more playful, follow the robot, or even take the robot in its mouth. Various children reported they would like to try with different toys, such as a dog bone, while others suggested that the robot should be faster, bigger and able to jump.

\subsection{Animal Computer Interaction for Playful Interactive Environments}

Play is an essential activity for all animal species, and it can help to improve the mental and physical wellbeing of animals in special circumstances. In this regard, technology can play a crucial role by creating suitable interactive spaces for animals to support playful interactions that can help to improve their wellbeing [258]. The field of Animal Computer Interaction is making a big effort to design such suitable interactive scenarios, which could be designed for humans and animals to play together, or for animals playing by themselves. 
In order to advance research regarding how technology could be used to create suitable technology for animal play, this work reviews the design, development and evaluation of two modalities of a playful interactive system for dogs. First, a remote system in which children control a tangible robot to play with a dog in a different location, followed by a pervasive system capable of controlling the robot autonomously to play with the dog without human intervention. This section compares the human-controlled system with the pervasive and autonomous system for dogs, with the aim of detecting usability issues, preferences and behavioral patterns that would help researchers to design better autonomous playful systems for dogs. This analysis has allowed to identify the next steps to be carried out for the development of intelligent playful environments for animals that give them engaging and adaptive experiences, helping to advance research within the ACI field.

\subsubsection{Participants}

The canine participants were dogs from Buma's Doggy Daycare, a local canine daycare facility in which dog owners can leave their dogs during working hours or holidays. All the dogs spend their time together inside the center, moving freely around a covered area of approximately 60 square meters. Of the 25 dogs that were introduced to the robot, only 2 were immediately let out of the interactive space as they seemed afraid of the device. A total of 14 dogs tried both the remote and the pervasive modalities and were considered for the observational study.

The play area in which the study took place was a delimited space of 4 meters long and 1.5 meters wide (see Figure 11.6). The robot was placed inside the area, while a Microsoft Kinect ${ }^{\circledR}$ sensor with a tripod was placed outside to record as much space as possible. Several plastic tubes were fixed to the ground over irregular spaces to prevent the ball from getting stuck in a corner or behind the dogs' beds.

\subsubsection{Interactive Playful Environment for Dogs: Remote vs. Pervasive}

In the remote interactive modality, one or two dogs participated in each session playing with a child, depending on the dogs' observed reactions. The robotic ball was placed inside the play area before the session started, and the dog participating was encouraged to enter the interactive space. Whenever a dog showed signs of distress or was seen to want to leave the interactive area, it was let outside and another took its place. Each session lasted for 5-10 minutes and was video recorded for further annotation to gather observational feedback. The set-up for this interactive modality is shown in Figure 11.6. 




Figure 11.6: Play area for the study.

The same procedure was followed for the autonomous interactive system: 5minute sessions, video recorded for later annotation, and the activity was stopped if the dog wanted to leave the interactive space or did not pay any attention to the device in the first 2-3 minutes. In this modality, the system should be able to detect both the dog and the robot device, and send commands to the robot to move it around the play area according to the dog's movements. The Microsoft Kinect sensor was placed in a higher position in order to get a better view, covering a smaller interaction area but reducing the possibility of the robot being hidden behind the dog or an object.

Previous work in ACI has explored non-wearable tracking systems for animals for play [257, 260, 323] or training purposes [190]. These studies produced systems capable of detecting different contextual information from the animal, ranging in complexity from simply detecting the animal's position [323] to classifying its body posture and body parts [190, 255, 257] and even its orientation/field of view $[257,260]$. As non-wearable tracking of an animal allows more natural and spontaneous interactions, this was the approach used in this study. Detecting the animals' movements and behavior without wearable devices generally requires supervised or semi-supervised training $[190,255]$ or imposes strong restrictions on the physical location, which has to be emptied of other objects that could obstruct or confuse the tracking algorithm. This makes it more difficult to adapt the system to different individuals and the person deploying the system must be experienced. The installation described in this study was aimed to accommodate to different dog participants each day, which made a supervised/semi-supervised approach 
unfeasible. Instead, an unsupervised interactive system capable of detecting only the animal's movements was preferred, as it would provide information on whether simpler contextual inference could provide similar results to the human-controlled modality in terms of animal engagement.

The Microsoft Kinect sensor used in the study provides both color and depth streams (Figure 11.7a). The pervasive interactive system processes the streams in real time to detect the dog's and robot's movements, and make the robot react accordingly. The first step of the image-processing algorithm consists of applying a background subtractor based on movement detection, for which the BackgroundSubtractorMOG2 algorithm from the EmguCV package for C\# was used. Once the background substractor has been applied, the remaining image shows the depth contour of the elements that have moved from the previous frame to the current one (Figure 11.7b). The robot's and the dog's contours must then be identified, assuming for the sake of simplicity that the bigger contour would be the dog's. In addition, at the start of the session the system allowed to configure the size thresholds of the robot and the dog to accommodate for minimal variations in the set-up height or lighting conditions that could affect the detection.
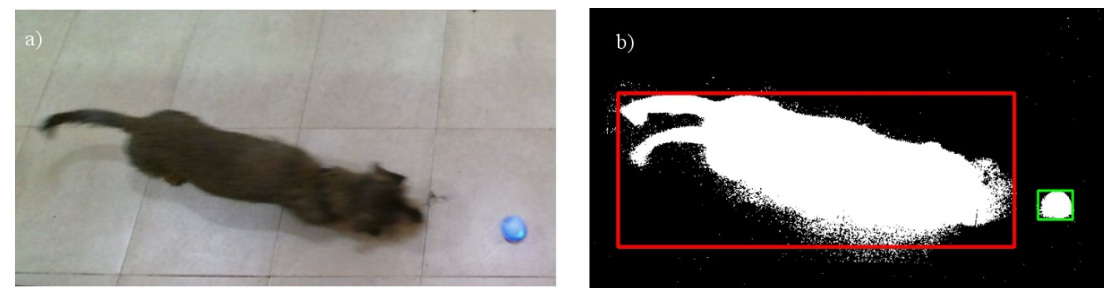

Figure 11.7: a) Color stream; b) Detection of dog and robot contours based on movement.

The decision system for controlling the robot was defined as follows: if the dog was not detected within the tracked area, the system sent a "spin" command to the robotic ball in order to make it rotate once around its axis. This action was repeated every few seconds until the dog was detected. When both the dog and the robot were detected within the tracked area, an orientation vector was traced between them, and the system sent a "move" command to the robot either to move it away or towards the dog, following the orientation vector between them. When neither the dog nor the robot was visible, a "random move" command was sent to the robot in case it was stuck in a hidden area. In addition, a more active approach was also implemented for dogs who did not seem interested in the normal approach described above. The dog behavioral expert suggested that some dogs might need more dynamic robot movements to get their attention. This approach was activated by the researcher after observing the dog's initial behavior with the device, and consisted of the same rules as in the normal approach, with a much 
shorter delay between the robot's movements. When the dog was not detected, the system sent a "random move" command instead of a "spin" command to the robot.

\subsubsection{Observational Results}

In general, even though the remote playful modality with children usually obtained a higher level of interactivity from the dogs, the dogs' behavioral patterns were constant in both modalities: if a dog was not interested in the activity it would not play in either the remote or pervasive modality. On the other hand, if a dog was seen to be interested in the activity, showing a playful attitude to the robot, it would interact in either the remote or the pervasive modality. The only difference was that in the remote modality, in which a child was controlling the robot, the interaction was usually more fluid and regular over time, as the child was better able to adapt to the dog's observed reactions. Four different behavioral patterns were identified in the sessions:

- Passive behavior: dogs showing passive behavior are not interested in the robot, staying in a calm and relaxed posture. In this scenario, the dog does not look at the robot while it moves (see Figure 11.8b), which could mean that the dog is interested in something else in the environment, the dog avoids eye contact with the robot, meaning it wants to avoid problems, or while staying perfectly calm it may not pay any attention to the device. Even when the robot approaches or touches the dog, it may not get any response.

- Alert behavior: alert behavior indicates the dog is aware of the robot, as it keeps eye contact with the device, however there are no signs of playful behavior or invitations to play on the dog's part. Instead, the dog changes its focus of interest between the robot and the environment, always keeping the robot in view, and moving opposite the robot when the device approaches.

- Playful behavior: in this behavioral pattern, the dog shows clear signs of enjoyment and playful behavior, such as wagging its tail or crouching with its front paws towards the robot in an invitation to play. Usually, the dynamics of play within this pattern are respectful and gentle. The dog looks at the robot while it moves, following it if it goes away or moving back if it approaches. Signs of excitement are shown when the robot moves, along with some barking. When the robot does not move at all, the dog stares at it expectantly for a while. However, if the robot does not move for a long period of time, the dog walks away and focuses on something else. When the robot starts moving again, even with just a gentle spin command to capture the dog's attention, the dog comes back again and restarts the playful interaction process. During the interaction, the dog might touch the robot repeatedly with its front paws. In several cases, the dog was seen to try 
to push the robot away with its snout, as if encouraging the robot to move and keep playing. Other examples of playful behavior include the dog trying to grab the robot with its mouth, or actually grabbing it and walking away. In this latter case, pervasive and ubiquitous spaces would allow a greater area of interaction to be defined, giving more control to the dogs in terms of when and where they prefer to play, and potentially even increasing the interest and attention of other dogs in the technology.

- Intense playful behavior: playful behaviors can happen with more or less intensity depending on the dog's age, character and mood. It was observed that some dogs displayed a more energetic play with the robot, always showing signs of playful behaviors as in the previous pattern described, e.g. wagging the tail. However, in this more intense play, other behaviors arose, such as jumping or running towards the robot, touching it repeatedly (see Figure 11.8a), throwing or kicking the robot away with their front paws, and trying to grab it with their mouth repeatedly. In this pattern of play, the dog did not wait much for the robot to move but instead actively started kicking the robot the moment it showed some minimal movement. In the remote playful activity, this meant that the child did not have a lot of control over the robot, as the dog was constantly moving it from one place to another. However, the children seemed to also enjoy this kind of interaction.
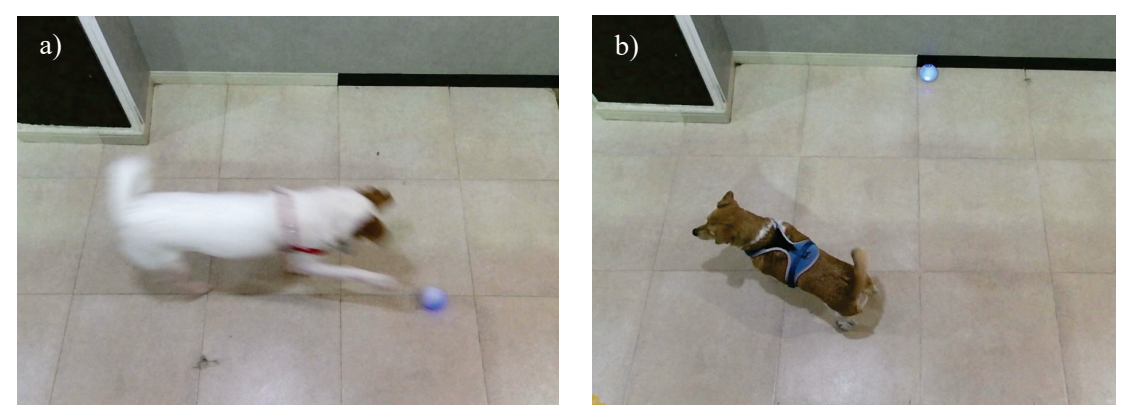

Figure 11.8: a) Dog interacting with robot; b) Dog not paying attention to the robot.

\subsubsection{Threats to Validity}

Although this study obtained interesting results, several precautions must be taken before generalizing these results to other contexts.

The presence of new people, such as researchers, and new elements in their environment, i.e. the technical installation and devices, usually triggered the dogs' interest. This excitement and interest could have diverted them from the playful 
activity towards the new things that were happening around them. In addition, the canine facility was open to the public during the study sessions, which occasionally meant that dog owners came to the facility to leave or collect their pets. This was also another source of distraction, as most of the dogs were curious about these events and approached the entrance, which in turn would get the attention of the rest of the group. Another aspect that could have conditioned the dogs' reactions towards the technology was their mood and their degree of familiarity with the other dogs at the facility on that day.

Some dogs required a brief introduction to the device, as their initial reaction was to avoid the robot as if they considered it a living creature. In these situations, two approaches could be followed. In the first attempt, the researcher took the robot in her hand and showed it to the dog, as if it were a regular plastic ball, letting the dog sniff it and become familiar with it. If this attempt was unsuccessful, the dog behavioral expert, who was known to the dogs, stood near to them during the activity to make them feel more comfortable. The first approach was successful for a few dogs, and could be included as part of the procedure to introduce the activity to shy or fearful individuals. The second approach did not produce any changes in the behavior of the dog, meaning that their interaction was the same, whether or not a human was present. It is hypothesized that dogs showing an alert behavioral pattern would probably need more time to become familiar with the robot in order to consider it friendly or a toy they could play with.

We believe all these threats to validity could be addressed by deploying a more permanent installation in the canine facility, which would allow the dogs to acclimatize to this new environment and in time they could even start to interact with the robot at their own will.

\subsection{Discussion}

\subsubsection{Remote Mobile Games for Children's Wellbeing}

The main goal of this work was to provide a mobile interactive experience that has the potential to improve the wellbeing of both children and animals while creating a stronger bond and sense of awareness in humans towards other species. After the observational results, and in light of the postquestionnaire results, the described remote playful application was found to be successful at providing an enjoyable and fun experience for children. During the sessions, the children were observed to be concentrated on the activity and showing physical signs of enjoyment, and they reported very positive results when asked about the experience and whether they would like to play with the animal again. Incorporating this activity into the daily routine of hospitalized children could thus be a promising opportunity to mitigate the stress produced by being hospitalized. Psychological assessment 
could be conducted in order to quantify the effects of this intervention on hospitalized children. This remote interactive experience could also be explored as a "distraction therapy" for pain or anxiety in child patients with high levels of pain or discomfort, or during painful procedures such as lumbar puncture, in which children need to stay still and avoid thinking about the procedure itself.

As reported in the questionnaire results, the majority of children said they would like to play with the animal again and with other children. This is a really positive result as it shows potential for the activity to become a social scenario. Communication and socialization within the hospital environment are very important aspects to improve children's mental wellbeing during their stay [159], especially in the case of long-term hospitalizations. These long stays at the hospital can produce emotional issues in children, sometimes due to feelings of loneliness or isolation for being separated from their friends or family [84, 320]. In this regard, the remote interaction with the animal could be considered as a social activity in which the child interacts with another being. However, this could be even more powerful if a social dimension with other children at the hospital was added. Creating a shared experience among hospitalized children could help them feel more connected to their new friends at the hospital. These social mobile environments could be deployed in several rooms at the hospital. Each child could be given a tablet or mobile device to connect to the streaming server and one would have control of the robotic device at a time, in a similar fashion as the authors proposed in [84]. In addition, chatting features could be added to the application to allow direct communication between children in different rooms. Another scenario could be to have multiple robot devices, each one controlled by a different child, all playing together at the same time with several dogs.

The sessions conducted with child patients from the oncology ward had to be conducted inside the child's room. This created a different set-up of the activity in which the parents and/or hospital teachers were also present during the session, and played an important and unexpected role in the interaction. The parents were able to encourage their children to interact with the dog in different ways, sharing an enjoyable experience at the hospital together. This activity could help not only the children, but also their parents, who are also under huge emotional pressure and stress [48]. Observing their children enjoy themselves and forgetting their condition for a while could have a positive impact not only on the children, but also on their parents in terms of reducing stress and anxiety. This, in turn, could help the children to improve their emotional wellbeing, as parents' feelings can affect the way in which children perceive their state. 


\subsubsection{Improving the Human-Animal Relationship}

In previous work by the authors, hospitalized children were asked to design playful shared games between themselves and an animal. It was observed that their perceptions about the animal improved after the design activity, and they were able to reflect on their designs to highlight potential pitfalls or scenarios that might not be as fun for the animals as it was for them [248].

The children's positive opinions of the animals in this previous study are corroborated in the postquestionnaire included in the present study. The children's answers showed positive likeability and perceived friendliness in the animals, and a will to learn more about the species and the animals playing with them. Different responses from the dog due to the animal's personality or mood at the time of the interaction could greatly affect the children's perceptions. However, in general they were very motivated towards exploring and trying different interactions to see if they could get the attention of the dog, even in the cases in which the dogs were not feeling playful. In addition, the children were able to acknowledge the cases in which dogs were not interested in the game, some of them even adding suggestions to improve the game or reasons why the dogs preferred not to interact.

The children were also capable of reflecting on the different implications this game could have for the animal in comparison to the experience they were having as human participants controlling the robot device. These design activities could thus be a promising tool to help raise awareness, foster critical thinking and improve relationships with different animal species. There is an opportunity to create playful learning activities that could also help to increase empathy by sharing an experience and learning about the animals' behavior and personality. This could also be envisioned for non-domestic animal species, such as endangered species in zoos or sanctuaries, to which children do not have easy access, and remote interactive scenarios could be a way of bringing them closer.

The level of awareness of the answers to Q12 also supports the postquestionnaire results. The children were capable of making suggestions to improve not only their personal experience of playing with the dog, but they were also thinking about the animals' wellbeing and enjoyment. Many of their suggestions addressed the addition of new features to the robot or elements to the game in order to make it more fun for the animal. They were able to reflect on the dogs' reactions in order to think about what kind of interactions could be more interesting for the animal. One of the children even reported that as the personality of the animal determines whether the dog would interact or not, it would be useful to have two dogs playing at the same time, one of them who likes to play and the other one who does not. In that way, the dog that plays could motivate the other dog to start playing as well, and at least the child who was controlling the robot could play with one of the dogs. It is surprising that this scenario proposed by a child participant was being used throughout the experiment whenever the dog therapist detected that 
the dog playing showed boredom or distress: either the dog was let out, or another dog entered the interactive space to see whether its company would foster playful behaviors.

\subsubsection{Shaping the Future of Pervasive Interactive Spaces for Animals}

A dog's reactions to an interactive system are subject to change due to different aspects. The most natural factor to consider when developing a meaningful interactive space for an animal would be its personality, which has a strong influence on how the dog perceives the interaction and the actions of the system. However, more subtle factors add a completely new range of variations to the animal's perception, such as the novelty of the environment, its mood, and contextual factors such as the presence of other dogs or humans. All the different behaviors observed during the interaction show the need for building adaptive pervasive systems that learn not only from the animal's movements but also from its individual preferences and personality. In addition, the system should also constantly adapt itself to the dog's reactions and not just at the beginning of the interaction based on a predefined personality. This would help to adapt the activity to the dog's mood on that specific day, as well as to other contextual information that could affect the interactivity levels of the experience.

A cornerstone aspect to consider in future developments of a pervasive system for dogs such as the one proposed here would be how to implement these adaptations to the dog's behavior without requiring too much training. In this regard, the data input from the remote interactive game with humans could shed some light on which features the system should consider, whether this is just the movement of the dog or more fine grained considerations, such as its body posture as proposed in other works [255]. When children were playing with the dogs, the movements they decided to perform with the robot were based on observations of the dog's reactions, a key aspect to making this modality more engaging for the animal involved. Such knowledge could be used to create appropriate models for the definition of an intelligent system for dogs' play.

The dogs showing playful behavioral patterns enjoyed the activity and showed willingness to go on playing when they were outside the playful area. Some of these demonstrations of interest included following the researcher during the setting up of the installation, entering the play area and walk straight to the robot whenever the door was open, and waiting outside the play area constantly looking at the robot, even trying to reach it with their paws. In parallel, there were also some dogs that showed interest in the robotic device but perhaps the interaction did not last long enough for them, or the set-up was not suitable for them to start playing. This shows potential for deploying this kind of pervasive environments for a longer period of time, which would allow shy dogs to acclimatize to the new elements in 
their surroundings, and could eventually lead to their wanting to participate in the interactive experience. In addition, a permanent installation of this type would give more control to the animals in terms of when and whether they would like to play. This could also help to improve the children's experience during a remote interactive game: the dogs would be more active and could give children a more rewarding experience if they see that the animal is enjoying with them.

As reported in the observational results and in the previous subsection, the children were also capable of identifying different animal behaviors, such as when the dog did not seem interested in the game. In the postquestionnaire, some of the children said they would like to know what the animal likes to do and how it wants to play, perhaps motivated by the identification of bored/non-interested behaviors. Based on the different personalities of the animals, a future machine learning system capable of extracting these features could learn how the animal prefers to play and provide clues to the children on their phone application on what to do during the game. In this way, the children could learn in which ways the interaction is beneficial for the animal, what the animals want and how we should interact with them. The game could also provide the children with hints or information about the animal, and the most suitable activity or way of interacting with them by detecting the animals' behaviors and actions during the game.

\subsection{Conclusion and Future Work}

This work has presented two main technological contributions, (1) a remote mobile interactive system for humans and animals which allows hospitalized children to remotely control a robot to play with a dog in a canine daycare facility, and (2) a pervasive interactive system capable of playing autonomously with a dog. The main aims of the playful interspecies system described here were to (1) help improve the children's wellbeing during their hospitalization, (2) foster a healthy relationship between children and animals by means of play, and (3) provide physical and mental stimulation for animals at home, in shelters or in daycare facilities.

The remote mobile system allowed hospitalized children who cannot receive the visits from therapy animals to interact with an animal in real time. This system was well received by both children and parents, and presented minimal interaction issues that could be easily resolved to make the interaction more fluid. The children were eager to propose additional features for the game, such as having access to different technological devices to play with the robot, or being able to observe other dogs with additional cameras. In order to assess the potential positive impact on the children's condition, e.g. stress or anxiety, a long term comparative study with a control group that has no access to the mobile application could be conducted. 
The results of the postquestionnaires showed positive levels of awareness and empathy towards the animals. The children were capable of identifying the benefits of these playful interactions for the dogs, as well as identifying which dogs were not very interested in the game and asking how they could engage the dog in the activity. This opens the door to the design of engaging educational activities aimed at fostering healthy relationships between humans and animals and to stimulate children's critical thinking and empathy.

Finally, a comparison between the child-controlled robot system and a pervasive system that autonomously plays with the dog showed that intelligent playful environments for animals need to learn from human interactions with the species in order to achieve the same levels of interest and engagement from the animal. The long-term deployment of these pervasive environments would verify whether dogs interact more as their confidence and familiarity with the system increases. This paper has allowed to identify the next steps to be carried out for the development of intelligent playful environments for animals that give them engaging and adaptive experiences, helping to advance research within the ACI field.

\section{Acknowledgments}

This work is supported by the European Development Regional Fund (EDRFFEDER) and Spanish MINECO (TIN2014-60077-R). The work of Patricia Pons is supported by the Spanish MECD (FPU13/03831). Special thanks to the dogs and children who participated in our study, as well as the dogs' owners and the children's families. The authors would also like to gratefully acknowledge the assistance of the teachers of the Unidad Pedagógica Hospitalaria La Fe and Oncología Pediátrica La Fe and also Olga and Astrid from Buma's Canine Daycare facility, for their invaluable support, collaboration and dedication. 



\section{Part V}

\section{Closure}





\section{Chapter 12}

\section{Discussion}

The research hypothesis of this work, as defined in Chapter 1, states that "Natural User Interfaces can be used effectively to create interactive and intelligent playful spaces for animals and humans, and they present an added value in terms of improving the animals' and humans' wellbeing, user experience and interrelationships". To prove this hypothesis, we have conducted a series of studies that have allowed us to explore step by step the domain area. The results and insights gathered from these studies led us to the final development of an interspecies playful system capable of playing autonomously with an animal, as well as allowing humans to remotely play with an animal using an interactive device.

First of all, this thesis motivated the need for more adaptive and pervasive solutions for technologically-mediated animal play. Chapter 3 formalized the definition, potential benefits and applications of Intelligent Playful Environments for Animals, analyzing the technological, ethical and behavioral considerations that should be taken into account when designing such spaces. Being ACI such a relatively new research area, there are yet many unexplored knowledge regions, specially due to the fact that animals cannot verbally communicate with us to explain their reasoning or decisions when interacting with a system. The creation of a fully intelligent and pervasive interactive space for animals is therefore complex and should be explored one step at a time. The final goal of this thesis is not the development of such a fully functional scenario, but rather the study and careful exploration of the different milestones that would allow researchers within ACI to know better how animals could use playful systems more naturally, how these systems could be tailored to different animal species adapting the system responses to the animals' intentions, and how they could help to improve the human-animal relationship (see Figure 1.1). 


\subsection{On NUIs for the Detection of Animals' Behaviors}

The first milestone defined in this thesis in order to explore the development of an intelligent playful environment for animals was to create a system based on NUIs to support the design and creation of such animal-centered interactive spaces. This design goal in itself can be broken down into the several steps described in Figure 1.1 and that have been studied in Chapters 4, 5 and 6 .

One of the first areas to explore when building a system that should adapt to the animals' interactions is how to implement these awareness mechanisms, i.e. create a system capable of detecting animals' basic behavior, using technology. This thesis proposed the use of Natural User Interfaces to the domain of animals' interactions with technology with the aim of allowing animals to behave spontaneously and without any physical limitation. Wearable devices are not a feasible approach for all animal species. Not all animals are used to wearing harnesses or collars, hence a wearable device might influence the behavior of the animal wearer [232]. In addition, it might not be safe for several species to wear such devices due to their strength, e.g. orangutans, as they could try to grab the device or break it. Non-wearable tracking mechanisms seemed therefore more adequate for the aforementioned purpose of providing a natural and unconstrained interaction.

Non-wearable tracking for building NUIs for humans has been a fruitful approach in HCI [69, 139, 140], with plenty of applications not only for play but also for remote working set-ups, among others. Despite its widespread presence in HCI, non-wearable tracking has been scarcely applied with animals, and it has been mainly focused on the analysis of color images for monitoring. However, the use of depth-sensors as the ones usually found in HCI literature could provide more complete information about the animal than conventional color cameras. Therefore, this thesis has proposed, designed and evaluated the first non-wearable tracking capable of detecting cats' body postures, body parts and orientation using a depth-sensor.

An initial evaluation of the system showed promising results, followed by an extensive and in-depth evaluation of the depth-based tracking system in two different approaches: a knowledge-based approach that would not require previous training of the system, and a supervised approach with a comparison of several feature vectors and classifiers that would require previous training of the system to build the cats' model. Both approaches showed very promising results and therefore tracking systems for cats based on depth information could effectively detect a cat's location and also use classification algorithms to recognize a cat's postures and body parts. Based on these positive results, the proposed tracking approach would be a suitable NUI to support animals' unconstrained interactions with an interactive playful environment. Moreover, the same approach could be used with animals of relatively similar anatomy and behavior with minimal adaptations. In 
an informal exploratory session, the system was used to track a different feline species, i.e. lions, providing very similar images of the animals' bodies that could be analyzed using the same approach proposed in this thesis with minor modifications to some parameters. In addition, other ACI researchers have followed our proposed approach to classify dog's postures without any wearable device [190, 191], which demonstrates the positive response of the community towards this novel approach and its potential applications.

Nevertheless, there is always a trade-off when deciding between unsupervised vs. supervised classification mechanisms. A comparative study between both classification approaches showed that the knowledge-based classification algorithm would not provide very accurate posture classification in all cases in its current state (see Chapter 6). Supervised classification algorithms, however, are capable of obtaining very high accuracy results as machine learning systems can identify characteristic features that are extremely difficult to identify by a human observer. However, supervised classification algorithms would require previous training of a model and hence are not suited for fast changing environments. Therefore, the decision on which approach to use should be carefully considered depending on the scenario. Supervised classification systems would be really useful for the detection of animals' behavior in scenarios in which the animals of a given species are usually the same individuals, e.g. in zoos or sanctuaries, or in which the size of different individuals within the species does not vary a lot, e.g. adult cows or horses. The supervised classification system in each case should be adapted to the corresponding behavior and analysis of the respective species. The proposed application scenarios could make use of supervised classifiers not only for playful activities but also for other application domains, such as providing very reliable information in monitoring systems for animal welfare and behavioral pattern recognition, or remote communication systems for dogs with occupations based on postures. This opens a very interesting line of research that however falls out of the scope of this thesis.

When dealing with fast-changing scenarios, i.e. animal participants would change from one session to another, or when the system has to be deployed by nonprogrammers, e.g. human owner of a pet, knowledge-based classification systems would be a better and faster approach. A knowledge-based classification technique should be able to work with different individuals without requiring further training of the system. This approach could be used when the system does not need to recognize fine-grained interactions. Knowledge-based classification system as the one proposed in this thesis provide satisfactory accuracy rates if other types of information derived from the body parts of the animal are required, such as determining the animal's field of view or just detecting a specific part of the animal such as the head. Hence, the rest of this thesis focused on the application of a knowledge-based approach for the detection of animals' natural user interactions. 
Several improvements have been proposed to increase the accuracy of the classification system, such as using background segmentation to better isolate the animal's contours from the rest of the environment, introducing temporal information to record paths and movement patterns, model the head's shape to provide more accurate information about the animal's field of view, etc. However, for the scope of this thesis, we focused on the incorporation of a functional and basic non-wearable tracking system into a playful environment. Once the suitability of the intelligent playful environment could be demonstrated, future research could explore how these improvements could be incorporated to improve the adaptation of the system.

\subsection{On NUIs Adaptation to Different Species and Contexts}

Once we had defined a NUI approach for tracking animals' spontaneous behavior within an interactive environment, the next step, defined in Figure 1.1, was to assess the suitability and flexibility of the proposal in terms of adapting to different animal species. It is essential to understand, observe and study how animals naturally play and behave with their surroundings in order to adapt the proposed non-wearable tracking system to those interactions. For this purpose, we have explored the tracking approach based on NUIs described in this thesis for two different animal populations and contexts: domestic cats and zoo-based orangutans.

In the case of domestic cats, we conducted an observational study to assess their preferences and spontaneous interactions with different technological artifacts and their observed engagement and interest (see Chapter 5). This observational study revealed different factors affecting their behavior and preferences towards different technological stimuli. Some of the aspects affecting their interactions were the familiarity with the device, age and size of the cats, and the ability of the stimuli to react accordingly and stay within the animal's field of view. Overall, small tangible robots worked well with cats provided that they were capable of simulating preylike behaviors. In cases in which the robot was bigger than the cat or when the cat was unsure about using the device, a traditional cat toy could be attached to the robot, which was more likely to trigger the cat's interest. These behaviors and play dynamics could be incorporated to the proposed tracking system based on depth images developed in Part II of this thesis.

Once a suitable non-wearable tracking mechanism for animals has been proposed for detecting the animals' behaviors and movements, this thesis has also explored how NUIs based on body tracking could be designed and used for a different species and in a different environmental context. The proposed depth-based tracking system has demonstrated to be flexible enough to be applied to species of similar 
anatomy, as other research groups have already implemented it for dogs [190, 191]. Taking one step further, the question would be how to create suitable NUIs based on non-wearable tracking mechanisms for species with very different anatomy and behavior (see Figure 1.1), such as orangutans. In this case, a research through design approach was conducted to propose and implement an interactive system for zoo enrichment with orangutans (see Chapters 7 and 8).

In both domestic and zoo contexts, tangible elements were considered as an effective tool for building up NUIs capable of tracking and augmenting the interaction of animals with such devices. In the case of domestic animals, tangible robots could move and adapt to the animal to create an engaging playful activity. In the case of zoos, orangutans have very different physical characteristics, and their zoo enclosure allows them to interact with any system in very different ways: standing, sitting, hanging down from the ceiling, swinging on a rope, etc. Therefore, the recognition system would differ to adapt to the species appropriate interactions. In addition, due to their strength, technological devices cannot be placed within the orangutans' enclosure. Hence, a better solution for this context was the detection and tracking of non-technological everyday objects, e.g. plastic balls. Then, the developed system was capable of producing different sounds based on the movements the orangutans perform with such objects. This would support interactions from very varied positions, as well as interactions with many different objects.

The developed system was intended to be used for the study of orangutans' preferences towards sound-based stimuli. However, initial evaluations of the system revealed that even with the iterative prototyping and testing process, orangutans could not easily map the produced digital stimuli to the actions they were performing. More adaptations should therefore be made to the system in order to evaluate the proposed goals. For example, the system was initially designed to cover a wide area of interaction, which led to difficulties in the tracking process when the object was too near or too far from the tracking device. In addition, when the orangutans walked away from the tracking device, the tracked object was hidden behind them and even though they were moving it away, the system could not emit any sound derived from this. Therefore, either the system should be conceived for a smaller and delimited area of interaction to guarantee tracking accuracy, such as in the case of interactive surfaces or walls, or the deployment would need additional tracking devices to cover different angles of the interactive space. Moreover, the physical set-up of the technological platform was also observed to be subject of interest for the orangutans. In this regard, designing a long-term or fixed installation could help to reduce the novelty factor and allow orangutans to get used to the system and start discovering the system at their own pace.

Overall, these studies over two populations have shown that non-wearable tracking systems can be used to create NUIs for different animal species. The key aspect 
would be to conduct proper ethnographic research, interviewing with humans in direct contact with the specific animal species, and iteratively adapt the designs to the observed results during trials. A Research through Design approach [14, $372,373]$, as proposed by other ACI researchers [81], seems very promising in this matter. The iterative designs and observations from the different trials, even though these are not gathered following a formal structure, can undoubtedly provide valuable insights and new knowledge. Hence, we as researchers need to adapt and acknowledge all the different ways in which our particular users provide us with new information to shape the technological systems aimed for them.

\subsection{On Human Participation in Playful Interactive Spaces for Animals}

Playful interactive environments for animals need to adapt to the animal species participating in the interactive experience, but humans could also be active participants in these scenarios. Humans have usually been assigned the role of providing technological artifacts for the animal to play with [5, 129, 224, 361]. However, this thesis proposes a more inclusive environment in which humans and animals would instead be fully participating players in interactive playful environments. Such an interactive playful system for animals and humans could help to improve the interrelationships and bonds between them by creating a shared space for their amusement. This thesis therefore has explored the co-design and development of Natural User Interfaces to include humans, and specifically children, as participants in interactive playful scenarios for animals. The aim of this part of the thesis is three-fold: to provide suitable NUIs for children's interactions within interactive spaces for animals, to help improving children's wellbeing and relationships with animals, and to study how interspecies playful interactive environments can help to improve the development of intelligent and adaptive systems for animals.

In order to design and develop suitable NUIs to support children's participation in interactive spaces for animals, we first need to look at how children want to interact within these interactive spaces. Although NUIs based on body tracking have been widely studied for adults [139, 226, 332], there are relatively few works regarding children's preferences with respect to full body interactions [28, 127]. Based on the previous work in this thesis validating the use of tangible elements to interact with specific animal species such as domestic animals, we have thus proposed the first elicitation study for children's control of tangible robots based on body gestures (Chapter 9). This study explored how children prefer to use their body language and gestures to control a tangible robot as the one domestic animals could have available to play with, using full body interactions, e.g. from moving one arm to walking or moving the whole body. The goal of the study was to propose a NUI so that children could easily manipulate these robots within an interactive space for animals, allowing them to focus on the animal participant 
and its interactions while moving freely around the space. The gestures defined by the children ranged from full body interactions, such as mimicking the robot's movements, to deictic gestures, such as pointing the desired path for the robot to follow, or even abstract gestures that did not have a physical mapping. This elicitation study resulted in the definition of a gestural language that accounts for the observed variations of the gestures by age and gender group, and had a good acceptance rate by children aged 6-12 years old. However, based on the time limitations and the results from a co-design activity in which children depicted their envisioned interactive playful environments with animals (Chapter 10), the implementation of the proposed gestural language fell out of the scope of this thesis, as motivated below.

In parallel to the design and elicitation of children's NUIs based on gestures within an interactive game with animals, the children's perception of such spaces is also essential to validate whether these gestural approach would be the most natural one for them in the first iteration of the system design. Therefore, as part of the user-centered approach of the proposed design of intelligent and interactive playful environments described in this thesis, one of the goals was to explore how children envision interspecies games with animals. Analyzing the human participants' vision of the interactive environment would allow us to create a more suitable and engaging experience for the human user, while helping to raise awareness towards keeping the system usable and adequate for the animals involved. For this purpose, we conducted a co-design activity with children in which they were asked to draw and describe how they would like to play with animals of different species. Children's co-designs showed traditional tablet based control of tangible robots as the preferred mechanism to interact with an animal. Therefore, it was decided that this would be the approach used to perform an initial evaluation of an interspecies playful system with animals. While this system shows promising results, the gestural language defined in the elicitation study described above could therefore be implemented and incorporated into the system to evaluate its usability and advantages towards the traditional mode.

This co-design activity also helped to explore another relevant aspect this thesis aimed to evaluate: the improvement of children's wellbeing and relationships with animals. This thesis has shown how both co-designing activities for the creation of playful systems for/with animals (Chapter 10), as well as the active participation in a playful activity with an animal (Chapter 11), positively reinforced children's perceptions of the animal participant. Children also showed capacity to reflect on the animals' needs, interactions and behaviors, which led them to suggest improvements to make the playful activity more fun and suitable for the animal. This shows how either designing for/playing with an animal could become an educational activity with benefits for both children and animals, even when they are not physically present in the same space. On one hand, these shared interactive spaces could help humans to stay in touch with their own pets, or help make animals in 
shelters visible so that they can find a home. These games could allow humans to interact with the animal, learn about their personality and behavior and develop empathy towards the individual, which could eventually lead to an adoption. On the other hand, remote interspecies games with animals in restricted environments such as zoos could play an educational role to raise awareness of the importance of wildlife preservation, or learning about endangered species behavior and abilities in an amusing way. On the animals' side, these playful experiences could improve the animal's wellbeing by introducing new forms of mental and physical stimulation adapted to each animal's context and preferences. In addition, considering the children population that participated in these two studies, i.e. hospitalized patients, remote interaction allowed them to be in contact with an animal and interact directly with it. These children, who cannot usually receive the visit of therapy animals, could at least have a glimpse of the manifold benefits animals can bring into our lives in stressful conditions such as a hospitalization process. Further studies should however be conducted in order to quantify properly the improvement of children's wellbeing thanks to the proposed remote interactive system.

Finally, insights gained from the successive studies described in this thesis have been combined to design and develop an interspecies playful environment for animals as well as for animals and humans based on NUIs (Chapter 11). This remote interactive game was aimed for dogs in a canine daycare facility, due to the additional physical and mental stimulation these dogs need during their stay. Several works within ACI have already studied dogs' interactions with tangible robots $[87,88,349]$, hence a tangible robot was the selected device for the interaction. The children's interactions within this system were defined by means of a mobile application for screen-based devices, and this application allowed the children to control the tangible robot in order to play remotely with the dog in the canine daycare facility. This system also included a basic intelligent mode in which the system played autonomously with the animal, and the two different approaches were compared.

One of the main initial goals of this thesis was that the system should be intuitive and the interaction as natural as possible for the animal, and this was accomplished and demonstrated in this last study, as the animals did not require any training to start interacting with the system in any of the two proposed modalities, i.e. remotely controlled robot by children or autonomous intelligent system. Dogs just entered the interactive play area and found their own ways for playing with the tangible robot: barking, touching it with the paws, looking at it, following it, etc. These interactions were not encouraged, they happened naturally and were not limited by any physical restriction. However, not all dogs have the same size and some of them were able to grab the device with their mouths, and others show preference towards carrying the device outside of the tracking area. This shows that more pervasive environments that cover a wider area would help to 
accommodate for such interactions, being able to track the device and the dog from many different angles and recognize these spontaneous interactions as well. In addition, this ability to expand the play area was also a suggestion several children made during their participation in the activity, as they would love to see more dogs playing and how they play in the whole facility. Regarding the tracking system, basic behavior was incorporated and the tracking was performed following a knowledge based approach that allowed to adapt to dogs of different sizes without training. However, there were scenarios in which the robotic device was occluded by the dog and could not be tracked, or when the dog moved too fast to be recognized properly by the system. More efforts should be directed towards improving a knowledge-based approach that could provide more reliability, and again having several cameras in a pervasive environment would be one possible solution to explore. Regarding the interactivity of both modalities, i.e. remote and system controlled, dogs were observed to interact more or with more frequency when a child was controlling the device. This could be because the observed information the child participant is capable of gathering when playing with the dog allows him to perform more natural movements of the robot, as well as smoother transitions from one movement to another, than the ones the autonomous system can achieve. Hence, we believe it is essential that intelligent systems learn from the interactions and play dynamics human participants add to interspecies playful activities mediated by technology. Only in this way a system could aim to be as effective as a human when providing suitable technological entertainment for the animal. 



\section{Chapter 13}

\section{Conclusions and Future Work}

This thesis has contributed to the study and development of playful interactive and intelligent environments for animals and humans, taking the first steps towards their realization. Throughout this manuscript, it has been demonstrated that Natural User Interfaces can be designed and applied to create suitable intelligent and interactive scenarios for different animal species. However, there are many research lines to investigate further, some of which have been proposed throughout the different chapters in this manuscript, highlighting here the most relevant ones.

First, this thesis has explored the definition, applications and benefits of playful interactive environments for animals (see Figure 1.1). There are several questions that could not be addressed within this work, but remain open to further exploration as the development of interactive environments for animals matures. For instance, one of the biggest concerns regarding technology for animal use is to provide animals with more agency over the technology designed for them. Intelligent playful systems for animals could address this issue by letting the animal decide when does it want to start playing. In the scope of this thesis, due to time limitations we were unable to deploy the physical set-up required for a long-term evaluation that would have allowed the exploration of animals' agency. However, with a more permanent installation, an intelligent system could be designed so that for example whenever the animal enters the interactive area, the tangible device starts moving. This could give the animal the opportunity to explore and get used to the system, and eventually decide at its own will when does it want to enter the interactive area to play with the robot, which will take us one step forward in the design of animal-centered systems.

Following an animal-centered approach in the design of intelligent playful scenarios has been a key factor in exploring the realization of these systems by means of Natural User Interfaces that account for the animals' spontaneous interactions. These NUIs were realized in this thesis with the design, development and evalu- 
ation of a promising and suitable non-wearable tracking system for the detection of animals' interactions, movements or body parts within an interactive playful scenario. It has been shown that several classification approaches, i.e. supervised and knowledge-based classification, of the detected animal's body parts and postures could be applied according to the context and required precision level. In this regard, there are several feasible updates that could help to improve the knowledge-based classification approach in terms of better adaptation to different animal individuals. For example, the tracking system could automatically detect the size of a cat, such as a kitten, and adapt the classifier to it without requiring the researcher or owner of the animal to perform manual labeling of the data to train a new classifier, which is a very time consuming task.

In addition, with the aim of assessing the suitability of the proposed approach based on NUIs, two different animal populations and contexts were studied, resulting in an observational study of domestic cats, and a Research through Design approach to propose and implement an interactive system for zoo enrichment with orangutans. The interactive modality chosen for both populations was based on tangible interactions with robotic devices, although different stimuli were triggered by the system in each case, depending on the elicited requirements and suitability: the cats could interact with a moving tangible robot, as opposed to the system emitting sounds as a result of the orangutan moving an object. Nevertheless, many other input and output stimuli remain to be studied as the field advances.

In addition, this work has explored the use of depth-based tracking in indoor scenarios, leaving the door open to the use of such an approach in outdoor areas. It is perhaps too soon to start developing this technology for outdoor scenarios, which usually entail more complexity regarding the environment, terrain, other animal species, etc. However, as the field and the technological systems mature, and other non-wearable tracking technologies are starting to flourish within ACI, such as thermal imaging [56] or drones [336], it is a matter of time before playful environments for animals could cover a wider area of interaction.

With the aim of including humans as active participants in these interactive systems for animals, several participatory design studies were conducted with children. Regarding the creation of suitable NUIs for human participation in playful environments for animals, an elicitation study was conducted in order to define a suitable gestural language for children to control a ground-based robot (see Chapter 9 ). The elicited gestural language was outside the scope of the thesis, however future work could include the implementation and evaluation of this gestural language for interspecies games. The evaluation of the gestural language could be compared with other types of interactive modalities, such as the ones proposed by the children during the co-design activity of playful experiences with animals (see Chapter 10), in terms of usability and engagement. In addition, the user interface design could greatly vary depending on whether the interaction between the human and the animal happens remotely or in the same physical space. It is 
hypothesized that screen-based interactions could probably work better for remote scenarios as the one developed in 11. However, when the animal and the human share the same physical space, having a device in their hands mediating the activity could limit the spontaneous interactions of children who perhaps would feel more natural to use their whole body to play with the animal, as in traditional games. Hence, this would be an interesting evaluation to provide the most suitable interactive modality in each case.

The insights gained through the studies involved in this thesis resulted in the design, development an evaluation of a basic playful environment capable of playing autonomously with an animal and a robotic device, as well as allowing a human to play remotely with the animal. In relation to the physical presence of the participants within the interactive space, the effects of a remote vs. same space scenario could be studied in both the human and the animal. In addition, with the aim of assessing quantitatively the effect of the playful activity on children's wellbeing, a psychological evaluation could be conducted. Moreover, in the case of animals, a long-term evaluation of the system in the canine daycare facility could help to observe changes in the interaction patterns over time, as well as produce a more reliable set of observations to assess improvements on the animals' wellbeing.

Overall, an immediate next step around the final design of an interspecies intelligent environment should be the design of a play system capable of learning from the children's interactions with the dog. In this way, the autonomous intelligent system could learn more complex behaviors that might feel more natural for the animal, thus increasing the animal's engagement and interest in the activity. In this regard, we are in the ideal technological conjunction for pursuing this goal, with advances such as machine learning or deep learning showing how systems are capable of extracting information from our data that we are not capable of reproducing ourselves.

There are still many questions that need to be addressed, but we hope that as the field advances, this manuscript can serve as a ground base to continue exploring how systems can provide suitable opportunities to support animals' playfulness. The final goal of such systems should be to help improving the mental and physical wellbeing of animals. In addition, extending such scenarios to include human participants would have many benefits. On one hand, it could help to create better interactive systems for the animals by learning about how humans play with them. On the other hand, natural user interfaces could help us to focus on these interspecies interactions by making technology as invisible as possible. Natural playful interactions could thus serve as the driving force to foster empathy towards different species, strengthening our bonds and improving our relationships with animals by learning more about them. 



\section{Bibliography}

[1] S. Akabane et al. "Puchi Planet: a tangible interface design for hospitalized children". In: Proceedings of the 2011 annual conference extended abstracts on Human factors in computing systems. 2011, pp. 1345-1350. DOI: 10. 1145/1979742.1979772 (cit. on p. 224).

[2] S. Akabane et al. "ZOOTOPIA: A Tangible and Accessible Zoo for Hospitalized Children". In: ACM SIGGRAPH ASIA 2010 Posters. New York, New York, USA: ACM Press, 2010, article no. 31. DOI: 10.1145/1900354. 1900388 (cit. on p. 224).

[3] H. Alborzi et al. "Designing StoryRooms: Interactive Storytelling Spaces for Children". In: Proceedings of the conference on Designing interactive systems processes, practices, methods, and techniques - DIS '00. Vol. 02. New York, New York, USA: ACM Press, 2000, pp. 95-104. DOI: 10.1145/ 347642.347673 (cit. on pp. 168, 170).

[4] J. Alcaidinho et al. "Leveraging Mobile Technology to Increase the Permanent Adoption of Shelter Dogs". In: Proceedings of the 17th International Conference on Human-Computer Interaction with Mobile Devices and Services - MobileHCI '15. New York, New York, USA: ACM Press, 2015, pp. 463-469. DOI: 10.1145/2785830.2785861 (cit. on p. 136).

[5] K. Alfrink et al. Pig Chase. Playing with Pigs project (cit. on pp. 23, 33, $34,58,63,198,225,254)$.

[6] M. Amat, T. Camps, S. L. Brech, and X. Manteca. "Separation anxiety in dogs: the implications of predictability and contextual fear for behavioural treatment". In: Animal Welfare 23.3 (2014), pp. 263-266. DOI: 10.7120/ 09627286.23.3.263 (cit. on p. 68). 
[7] S. Amir, A. Zamansky, and D. van der Linden. "K9-Blyzer - Towards Video-Based Automatic Analysis of Canine Behavior". In: Proceedings of the Fourth International Conference on Animal-Computer Interaction ACI2017. New York, New York, USA: ACM Press, 2017, pp. 1-5. DOI: $10.1145 / 3152130.3152142$ (cit. on pp. 23, 39).

[8] J. Amores, X. Benavides, and L. Shapira. "TactileVR: Integrating Physical Toys into Learn and Play Virtual Reality Experiences". In: Proceedings of the 2016 CHI Conference Extended Abstracts on Human Factors in Computing Systems - CHI EA '16. New York, New York, USA: ACM Press, 2016, pp. 100-106. DOI: 10.1145/2851581.2889438 (cit. on p. 222).

[9] A. N. Antle, G. Corness, and M. Droumeva. "What the body knows: Exploring the benefits of embodied metaphors in hybrid physical digital environments". In: Interacting with Computers 21.1-2 (2009), pp. 66-75. DOI: 10.1016/j.intcom.2008.10.005 (cit. on p. 169).

[10] Apps for Apes. https://redapes.org/multimedia/apps-for-apes/. [Online; accessed 16-April-2018]. 2016 (cit. on pp. 23, 33, 97, 99, 148, 154, 224).

[11] F. Aspling and O. Juhlin. "Theorizing animal-computer interaction as machinations". In: International Journal of Human-Computer Studies 98 (2017), pp. 135-149. DOI: 10.1016/j.ijhcs.2016.05.005 (cit. on pp. 19, 44).

[12] S. B. Barker and K. S. Dawson. "The effects of animal-assisted therapy on anxiety ratings of hospitalized psychiatric patients". In: Psychiatric services 49.6 (1998), pp. 797-801 (cit. on pp. 70, 165, 223).

[13] S. B. Barker, A. K. Pandurangi, and A. M. Best. "Effects of animal-assisted therapy on patients' anxiety, fear, and depression before ECT". In: The journal of ECT 19.1 (2003), pp. 38-44 (cit. on pp. 165, 223).

[14] D. A. Basballe and K. Halskov. "Dynamics of research through design". In: Proceedings of the Designing Interactive Systems Conference on - DIS '12. New York, New York, USA: ACM Press, 2012, pp. 58-67. DOI: 10.1145/ 2317956.2317967 (cit. on p. 254).

[15] S. Baskin, S. Anavi-goffer, and A. Zamansky. "Digital Game Design for Canines: Getting to Know Your User". In: Proceedings of the British Human Computer Interaction Conference - Animal Computer Interaction Workshop. 2015, pp. 1-4 (cit. on p. 98). 
[16] S. Baskin, S. Anavi-goffer, and A. Zamansky. "Serious Games: Is Your User Playing or Hunting?" In: 14th International Conference on Entertainment Computing (ICEC 2015). 2015, pp. 475-481 (cit. on pp. 98, 118).

[17] S. Baskin and A. Zamansky. "The Player is Chewing the Tablet!" In: Proceedings of the 2015 Annual Symposium on Computer-Human Interaction in Play - CHI PLAY '15. New York, New York, USA: ACM Press, 2015, pp. 463-468. DOI: 10.1145/2793107.2810315 (cit. on pp. 3, 21, 23, 33-35, $96,98,200)$.

[18] S. Baskin, A. Zamansky, and V. Kononova. "Exploring human perceptions of dog-tablet playful interactions". In: Proceedings of the Third International Conference on Animal-Computer Interaction - ACI '16. New York, New York, USA: ACM Press, 2016, pp. 1-4. DOI: 10.1145/2995257.3012023 (cit. on pp. 200, 225).

[19] P. Bateson and P. Martin. Play, Playfulness, Creativity and Innovation. New York: Cambridge University Press, 2013 (cit. on pp. 48, 222).

[20] J. Bednarik and D. Herman. "Human gesture recognition using top view depth data obtained from Kinect sensor". In: Excel@FIT - Student Conference on Innovation, Technology and Science in IT. 2015, pp. 1-8 (cit. on pp. 86, 124).

[21] M. Bekoff and C. Allen. "Intentional communication and social play: how and why animals negotiate and agree to play". In: Animal play: Evolutionary, comparative, and ecological perspectives. Ed. by M. Bekoff and J. A. Byers. New York: Cambridge University Press, 1997, pp. 97-114 (cit. on p. 55).

[22] T. Belpaeme et al. "Multimodal Child-Robot Interaction: Building Social Bonds". In: Journal of Human-Robot Interaction 1.2 (2013), pp. 33-53. DOI: 10.5898/JHRI.1.2.Belpaeme (cit. on p. 170).

[23] H. Benko, A. D. Wilson, F. Zannier, and H. Benko. "Dyadic projected spatial augmented reality". In: Proceedings of the 27th annual ACM symposium on User interface software and technology - UIST '14. 2014, pp. 645-655. DOI: $10.1145 / 2642918.2647402$ (cit. on pp. $82,124,168$ ).

[24] R. Bergmüller. "Animal personality and behavioural syndromes". In: $A n$ imal Behaviour: Evolution and Mechanisms. 2010, pp. 587-621. DOI: 10 . 1007/978-3-642-02624-9_19 (cit. on p. 100). 
[25] S. Blom, M. Boere-Boonekamp, and R. Stegwee. "Social connectedness through ICT and the influence on wellbeing: the case of the CareRabbit". In: Studies in health technology and informatics 169 (2011), pp. 78-82. DOI: 10.3233/978-1-60750-806-9-78 (cit. on p. 224).

[26] S. Blom, R. Stegwee, and M. Boere-Boonekamp. "The CareRabbit". In: Proceedings of the 28th Annual European Conference on Cognitive Ergonomics - ECCE '10. August. ACM Press, 2010, pp. 355-356. DOI: 10.1145/1962300. 1962383 (cit. on p. 224).

[27] A. F. Bobick et al. "The KidsRoom: A Perceptually-Based Interactive and Immersive Story Environment". In: Presence: Teleoperators and Virtual Environments 8.4 (1999), pp. 367-391. DOI: 10.1162/105474699566297 (cit. on pp. 170, 222).

[28] A. Bonarini, F. Clasadonte, F. Garzotto, and M. Gelsomini. "Blending robots and full-body interaction with large screens for children with intellectual disability". In: Proceedings of the 14th International Conference on Interaction Design and Children - IDC '15. New York, New York, USA: ACM Press, 2015, pp. 351-354. DOI: 10.1145/2771839.2771914 (cit. on pp. 168, 170, 254).

[29] H. Boostrom. Problem-Solving with Orangutans (Pongo pygmaeus and Pongo abelii) and Chimpanzees (Pan troglodytes): Using the IPAD to Provide Novel Enrichment Opportunities. Tech. rep. 2013 (cit. on p. 148).

[30] A. Bozkurt et al. "Toward Cyber-Enhanced Working Dogs for Search and Rescue". In: IEEE Intelligent Systems 29.6 (2014), pp. 32-39. DOI: 10.1109/ MIS.2014.77 (cit. on pp. 23, 28, 29, 81, 96, 99, 121, 123).

[31] J. W. Bradbury and S. L. Vehrencamp. Principles of Animal Communication. Sinauer Associates, 1998, p. 697 (cit. on p. 5).

[32] L. Brent and D. Weaver. "The physiological and behavioral effects of radio music on singly housed baboons". In: Journal of Medical Primatology 25.5 (1996), pp. 370-374. DOI: 10.1111/j.1600-0684.1996.tb00031.x (cit. on p. 155).

[33] W. R. Britt et al. "An embedded system for real-time navigation and remote command of a trained canine". In: Personal and Ubiquitous Computing 15.1 (2011), pp. 61-74. DOI: 10.1007/s00779-010-0298-4 (cit. on pp. 21, 27, 29). 
[34] R. Brugarolas et al. "Behavior recognition based on machine learning algorithms for a wireless canine machine interface". In: 2013 IEEE International Conference on Body Sensor Networks. IEEE, 2013, pp. 1-5. DOI: 10.1109/BSN.2013.6575505 (cit. on pp. 23, 29, 81, 121, 123).

[35] R. Buchanan. "Design Research and the New Learning". In: Design Issues 17.4 (2001), pp. 3-23. DOI: 10.1162/07479360152681056 (cit. on p. 19).

[36] G. M. Burghardt. The genesis of animal play. Testing the limits. Cambridge, MA, USA: MIT Press, 2006 (cit. on p. 55).

[37] C. Byrne, L. Freil, T. Starner, and M. M. Jackson. "A method to evaluate haptic interfaces for working dogs". In: International Journal of Human Computer Studies 98 (2017), pp. 196-207. DoI: 10.1016/j.ijhcs.2016.04.004 (cit. on pp. 23, 27, 29).

[38] C. Byrne et al. "Predicting the Suitability of Service Animals Using Instrumented Dog Toys". In: Proceedings of the ACM on Interactive, Mobile, Wearable and Ubiquitous Technologies 1.4 (2018), pp. 1-20. DOI: 10.1145/ 3161184 (cit. on pp. 23, 26).

[39] M. Campion. History of Eye Tracking Studies and Technology. Look Tracker. https: / / www.looktracker.com/blog/eye-tracking-technology/the-historyof-eye-tracking-studies-and-technology/ (cit. on p. 37).

[40] F. Carpio et al. "Beyond Production Indicators: A Novel Smart Farming Application and System for Animal Welfare". In: Proceedings of the Fourth International Conference on Animal-Computer Interaction - ACI201\%. New York, New York, USA: ACM Press, 2017, 7:1-7:11. DOI: 10.1145/3152130. 3152140 (cit. on pp. 23, 30).

[41] M. Carter, S. Webber, and S. Sherwen. "Naturalism and ACI: Augmenting Zoo Enclosures with Digital Technology". In: Proceedings of the 12th International Conference on Advances in Computer Entertainment Technology - $A C E$ '15. New York, New York, USA: ACM Press, 2015, 61:1-61:5. DOI: 10.1145/2832932.2837011 (cit. on pp. 21, 118, 154).

[42] A. Catalá et al. "A meta-model for dataflow-based rules in smart environments: Evaluating user comprehension and performance". In: Science of Computer Programming 78.10 (2013), pp. 1930-1950. DOI: 10.1016/j.scico. 2012.06.010 (cit. on p. 68). 
[43] J. R. Cauchard, J. L. E, K. Y. Zhai, and J. A. Landay. "Drone \& me: An Exploration Into Natural Human-Drone Interaction". In: Proceedings of the 2015 ACM International Joint Conference on Pervasive and Ubiquitous Computing - UbiComp '15. New York, New York, USA: ACM Press, 2015, pp. 361-365. DOI: 10.1145/2750858.2805823 (cit. on pp. 168, 172, 194).

[44] A. D. Cheok et al. "Metazoa Ludens: Mixed-Reality Interaction and Play for Small Pets and Humans". In: IEEE Transactions on Systems, Man, and Cybernetics - Part A: Systems and Humans 41.5 (2011), pp. 876-891. DOI: 10.1109/TSMCA.2011.2108998 (cit. on pp. 58, 63, 91, 92).

[45] C. Q. Choi. Chimps Make Spears and Hunt Bushbabies. 2007 (cit. on p. 148).

[46] A. W. Clay et al. "The use of technology to enhance zoological parks". In: Zoo Biology 30.5 (2011), pp. 487-497. DOI: 10.1002/zoo.20353 (cit. on p. 3).

[47] CleverPet. CleverPet: Engage Idle Paws (cit. on pp. 17, 25).

[48] E. Commodari. "Children staying in hospital: a research on psychological stress of caregivers." In: Italian journal of pediatrics 36 (2010), p. 40. DOI: 10.1186/1824-7288-36-40 (cit. on p. 241).

[49] S. Connell, P.-Y. Kuo, L. Liu, and A. M. Piper. "A Wizard-of-Oz elicitation study examining child-defined gestures with a whole-body interface". In: Proceedings of the 12th International Conference on Interaction Design and Children - IDC '13. New York, New York, USA: ACM Press, 2013, pp. 277 280. DOI: $10.1145 / 2485760.2485823$ (cit. on pp. 171, 192, 193).

[50] B. Costello and E. Edmonds. "A Study in Play, Pleasure and Interaction Design". In: Proceedings of the 2007 conference on Designing pleasurable products and interfaces. August. 2007, pp. 76-91. DOI: 10.1145/1314161. 1314168 (cit. on p. 51).

[51] M. Csikszentmihalyi. Beyond Boredom and Anxiety. The Experience of Play in Work and Games. Jossey-Bass Publishers, 1975 (cit. on p. 51).

[52] C. Darwin. On the Origin of Species by Means of Natural Selection, or the Preservation of Favoured Races in the Struggle for Life. John Murray, 1859 (cit. on p. 18).

[53] J. Derboven, M. Van Mechelen, and K. Slegers. "Multimodal Analysis in Participatory Design with Children". In: Proceedings of the 33rd Annual ACM Conference on Human Factors in Computing Systems - CHI '15. 
New York, New York, USA: ACM Press, 2015, pp. 2825-2828. DOI: 10. 1145/2702123.2702475 (cit. on p. 171).

[54] A. K. Dixon. "Ethological strategies for defence in animals and humans: Their role in some psychiatric disorders". In: British Journal of Medical Psychology 71.4 (1998), pp. 417-445. DOI: 10.1111/j.2044-8341.1998. tb01001.x (cit. on p. 16).

[55] H. Dong, A. Danesh, N. Figueroa, and A. E. Saddik. "An Elicitation Study on Gesture Preferences and Memorability Toward a Practical Hand-Gesture Vocabulary for Smart Televisions". In: IEEE Access 3 (2015), pp. 543-555. DOI: 10.1109/ACCESS.2015.2432679 (cit. on pp. 168, 172).

[56] R. Dong et al. "Supporting animal welfare with automatic tracking of giraffes with thermal cameras". In: Proceedings of the 29th Australian Conference on Computer-Human Interaction - OZCHI '17. 2017, pp. 386-391. DOI: $10.1145 / 3152771.3156142$ (cit. on pp. $23,38,40,260$ ).

[57] A. Druin. "Cooperative inquiry: Developing New Technologies for Children with Children". In: Proceedings of the SIGCHI conference on Human factors in computing systems the CHI is the limit - CHI'99 14.99 (1999), pp. 592599. DOI: 10.1145/302979.303166 (cit. on pp. 169, 199).

[58] A. Druin. "The role of children in the design of new technology". In: Behaviour \& Information Technology 21.1 (2002), pp. 1-25. DOI: 10.1080/ 01449290110108659 (cit. on pp. 171, 199).

[59] A. Druin et al. "Children as our technology design partners". In: The design of children's technology. Ed. by A. Druin. Morgan Kaufman, San Francisco, CA, 1999, pp. $51-72$ (cit. on pp. 7, 169, 171, 199).

[60] H. Dubberly, P. Pangaro, and U. Haque. "What is interaction? Are there different types?" In: Interactions 16.1 (2009), pp. 69-75. DOI: $10.1145 /$ 1456202.1456220 (cit. on p. 19).

[61] W. van Eck and M. Lamers. "Animal Controlled Computer Games: Playing Pac-Man Against Real Crickets". In: International Conference on Entertainment Computing (ICEC) 2006, Lecture Notes in Computer Science (LNCS) 4161. 2006, pp. 31 -36. DOI: 10.1007/11872320 (cit. on pp. 23, 27, $43)$.

[62] W. van Eck and M. H. Lamers. "Player Expectations of Animal Incorporated Computer Games". In: Intelligent Technologies for Interactive En- 
tertainment. INTETAIN 2017. Lecture Notes of the Institute for Computer Sciences, Social Informatics and Telecommunications Engineering. Vol. 215. 2018, pp. 1-15. DOI: $10.1007 / 978-3-319-73062-2 \_1$ (cit. on pp. 23,27$)$.

[63] B. English and A. Howard. "Increasing Motor Learning During Hand Rehabilitation Exercises Through the Use of Adaptive Games: A Pilot Study". In: 3rd International Workshop on Intelligent Digital Games for Empowerment and Inclusion (IDGEI 2015). 2015, pp. 2-7 (cit. on p. 96).

[64] Enrichment. Association of Zoos and Aquariums. https://www.aza.org/ enrichment/ (cit. on p. 154).

[65] Enrichment for Primates. http://nationalzoo.si.edu/animals/primates / enrichment/default.cfm. [Online; accessed 16-April-2018]. 2016 (cit. on p. 148).

[66] J. Epps, S. Lichman, and M. Wu. "A study of hand shape use in tabletop gesture interaction". In: CHI '06 extended abstracts on Human factors in computing systems - CHI EA '06. New York, New York, USA: ACM Press, 2006, pp. 748-753. DOI: 10.1145/1125451.1125601 (cit. on p. 172).

[67] T. Faragó et al. "Social behaviours in dog-owner interactions can serve as a model for designing social robots". In: Interaction Studies 15.2 (2014), pp. 143-172 (cit. on p. 37).

[68] J. Fast. "Body Language". In: (1971), p. 189 (cit. on p. 5).

[69] A. R. Fender, H. Benko, and A. Wilson. "MeetAlive : Room-Scale OmniDirectional Display System for Multi-User Content and Control Sharing". In: Proceedings of the 2017 ACM International Conference on Interactive Surfaces and Spaces. 2017, pp. 106-115. DOI: $10.1145 / 3132272.3134117$ (cit. on pp. 168, 250).

[70] R. A. S. Fernandez et al. "Natural user interfaces for human-drone multimodal interaction". In: 2016 International Conference on Unmanned Aircraft Systems (ICUAS). IEEE, 2016, pp. 1013-1022. DOI: 10.1109/ICUAS. 2016.7502665 (cit. on p. 173).

[71] S. L. Filan and R. H. Llewellyn-Jones. "Animal-assisted therapy for dementia: a review of the literature". In: International psychogeriatrics / IPA 18.4 (2006), pp. 597-611. DOI: 10.1017/S1041610206003322 (cit. on p. 70). 
[72] Fitbark. FitBark Dog Activity Monitor: Healthy Together (cit. on pp. 17, $23,28,41)$.

[73] C. Frauenberger, J. Good, and W. Keay-Bright. "Designing Technology for Children with Special Needs - Bridging Perspectives through Participatory Design". In: CoDesign 7.March 2015 (2011), pp. 1-28. DOI: 10.1080/ 15710882.2011.587013 (cit. on p. 199).

[74] L. Freil et al. "Canine-Centered Computing". In: Foundations and Trends in Human-Computer Interaction 10.2 (2017), pp. 87-164. DOI: 10.1561/ 1100000064 (cit. on pp. 20, 43).

[75] F. French, C. Mancini, and H. Sharp. "Designing Interactive Toys for Elephants". In: Proceedings of the 2015 Annual Symposium on ComputerHuman Interaction in Play - CHI PLAY '15. New York, New York, USA: ACM Press, 2015, pp. 523-528. DOI: 10.1145/2793107.2810327 (cit. on pp. 21, 24, 98, 222).

[76] F. French, C. Mancini, and H. Sharp. "Exploring methods for interaction design with animals: a case-study with Valli". In: Proceedings of the Third International Conference on Animal-Computer Interaction - ACI '16. New York, New York, USA: ACM Press, 2016, 3:1-3:5. DOI: 10.1145/2995257. 2995394 (cit. on pp. 24, 26, 42, 198).

[77] F. French, C. Mancini, and H. Sharp. "High tech cognitive and acoustic enrichment for captive elephants". In: Journal of Neuroscience Methods 300 (2018), pp. 173-183. DOI: 10.1016/j.jneumeth.2017.09.009 (cit. on pp. $23,24,27)$.

[78] F. French, C. Mancini, and H. Sharp. "Playful UX for Elephants". In: Measuring Behavior 2016. 2016, pp. 470-473 (cit. on pp. 96, 198).

[79] F. French, C. Mancini, H. Sharp, and N. Smith. "Designing smart toys for the cognitive enrichment of elephants". In: First Symposium on Intelligent Systems for Animal Welfare, Proceedings of the 50th Convention on Artificial Intelligence and Simulation of Behaviour. 2014, pp. 1-5 (cit. on pp. 4, $98,118)$.

[80] F. French et al. "Don't cut to the chase: hunting experiences for zoo animals and visitors". In: Proceedings of the Third International Conference on Animal-Computer Interaction - ACI '16. New York, New York, USA: ACM Press, 2016, pp. 1-6. DOI: 10.1145/2995257.3014066 (cit. on p. 138). 
[81] F. French, H. Sharp, F. French, and H. Sharp. "Exploring Research through Design in Animal Computer Interaction Exploring Research through Design in Animal Computer Interaction". In: Proceedings of the Fourth International Conference on Animal-Computer Interaction (ACI 2017). November. 2017. DOI: $10.1145 / 3152130.3152147$ (cit. on pp. 7,254 ).

[82] T. Friend. Animal talk: breaking the codes of animal language. New York: Atria Books, 2004, p. 288 (cit. on p. 5).

[83] M. García-Herranz, P. A. Haya, and X. Alamán. "Towards a Ubiquitous End-User Programming System for Smart Spaces". In: Journal Of Universal Computer Science 16.12 (2010), pp. 1633-1649. DOI: 10.3217/jucs-01612-1633 (cit. on p. 68).

[84] F. Garcia-Sanjuan, J. Jaen, and S. Jurdi. "Towards Encouraging Communication in Hospitalized Children through Multi-Tablet Activities". In: Proceedings of the XVII International Conference on Human Computer Interaction. 2016, pp. 29.1-29.4. DOI: 10.1145/2998626.2998658 (cit. on pp. $219,224,241)$.

[85] F. Garcia-Sanjuan, J. Jaen, and V. Nacher. "Tangibot: A tangible-mediated robot to support cognitive games for ageing people-a usability study". In: Pervasive and Mobile Computing 34 (2017), pp. 91-105. DOI: http://dx. doi.org/10.1016/j.pmcj.2016.08.007 (cit. on p. 96).

[86] F. Garcia-Sanjuan, J. Jaen, V. Nacher, and A. Catala. "Design and evaluation of a tangible-mediated robot for kindergarten instruction". In: Proceedings of the 12th International Conference on Advances in Computer Entertainment Technology - ACE '15. New York, New York, USA: ACM Press, 2015, pp. 1-11. DOI: 10.1145/2832932.2832952 (cit. on pp. 168, 170).

[87] A. Gergely, A. B. Compton, R. C. Newberry, and Á. Miklósi. "Social Interaction with an "unidentified Moving Object" Elicits A-Not-B Error in Domestic Dogs". In: PLoS ONE 11.4 (2016), pp. 1-12. DOI: 10.1371/journal. pone.0151600 (cit. on pp. 23-25, 42, 256).

[88] A. Gergely, E. Petró, J. Topál, and A. Miklósi. "The emergence of social interaction between Dog and an Unidentified Moving Object (UMO)". In: 50th Annual Convention of the Society for the Study of Artificial Intelligence and the Simulation of Behaviour. 2014 (cit. on pp. 23, 24, 256).

[89] A. Geurtsen, M. H. Lamers, and M. J. M. Schaaf. "Interactive Digital Gameplay Can Lower Stress Hormone Levels in Home Alone Dogs: A 
Case for Animal Welfare Informatics". In: 14th International Conference on Entertainment Computing (ICEC 2015). Ed. by K. Chorianopoulos et al. Vol. 9353. Lecture Notes in Computer Science. Cham: Springer International Publishing, 2015, pp. 238-251. DOI: 10.1007/978-3-319-24589-8 (cit. on pp. 19, 21, 23-25, 42).

[90] E. Gillam. "An Introduction to Animal Communication". In: Nature Education Knowledge 10.3 (2011), p. 70 (cit. on p. 5).

[91] O. Gillman. Tie me kangaroo drone sport: Incredible moment marsupial punches flying camera out of the sky. 2014 (cit. on p. 3).

[92] J. Gindling et al. "LEGOsheets: a rule-based programming, simulation and manipulation environment for the LEGO Programmable Brick". In: Proceedings of Symposium on Visual Languages. IEEE Comput. Soc. Press, pp. 172-179. DOI: 10.1109/VL.1995.520806 (cit. on p. 170).

[93] K. R. Ginsburg. "The Importance of Play in Promoting Healthy Child Development and Maintaining Strong Parent-Child Bonds and the Committee on Communications and the Committee on Psychosocial Aspects of Child and Family Health". In: Pediatrics 119 (2007), pp. 182-191. DOI: 10.1542/peds.2006-2697 (cit. on p. 222).

[94] E. Goffman. Strategic Interaction. University of Pennsylvania Press, 1971, p. 160 (cit. on p. 19).

[95] B. Gonzalez, J. Borland, and K. Geraghty. "Whole body interaction for child-centered multimodal language learning". In: Proceedings of the 2nd Workshop on Child, Computer and Interaction - WOCCI '09. New York, New York, USA: ACM Press, 2009, pp. 1-5. DOI: 10.1145/1640377.1640381 (cit. on p. 170).

[96] D. Goode. Playing with my dog Katiee: An Ethnomethodological Study of Dog- Human Interaction. Purdue University Press, 2006, p. 180 (cit. on p. 19).

[97] K. Goris, J. Saldien, and D. Lefeber. "Probo, a testbed for human robot interaction". In: 2009 4th ACM/IEEE International Conference on HumanRobot Interaction (HRI). 2009, pp. 253-254. DOI: 10.1145/1514095.1514162 (cit. on p. 224).

[98] K. Goris, J. Saldien, I. Vanderniepen, and D. Lefeber. "The huggable robot Probo, a multi-disciplinary research platform". In: Communications in Com- 
puter and Information Science 33 (2009), pp. 29-41. DOI: 10.1007/978-3642-03558-6_4 (cit. on p. 224).

[99] R. A. Grant and B Hewitt. "Use Of Image Processing Techniques To Quantify Sensory and Motor Behaviors in Rodents By Measuring Whisker Movements". In: Measuring Behavior 2016. 2016, pp. 20-22 (cit. on pp. 23, 39).

[100] K. Grønbæk et al. "Interactive Floor Support for Kinesthetic Interaction in Children Learning Environments". In: Human-Computer Interaction INTERACT 200\%. Lecture Notes in Computer Science. Vol. 4663. 2007, pp. 361-375. DOI: 10.1007/978-3-540-74800-7_32 (cit. on pp. 168, 170, $222)$.

[101] M. L. Guha et al. "Working with young children as technology design partners". In: Communications of the ACM 48.1 (2005), pp. 39-42. DOI: 10.1145/1039539.1039567 (cit. on pp. 171, 199).

[102] K. Guo et al. "Left gaze bias in humans, rhesus monkeys and domestic dogs". In: Animal Cognition 12.3 (2009), pp. 409-418. DOI: 10.1007/s10071008-0199-3 (cit. on p. 37).

[103] R. Gupfinger and M. Kaltenbrunner. "Sonic Experiments with Grey Parrots: A Report on Testing the Auditory Skills and Musical Preferences of Grey Parrots in Captivity". In: Proceedings of the Fourth International Conference on Animal-Computer Interaction (ACI 2017). 2017, 3:1-3:6. DOI: $10.1145 / 3152130.3152137$ (cit. on pp. 23, 24).

[104] J. Haladjian, Z. Hodaie, S. Nüske, and B. Brügge. "Gait Anomaly Detection in Dairy Cattle". In: Proceedings of the Fourth International Conference on Animal-Computer Interaction (ACI 2017). 2017, 8:1-8:8. DOI: 10.1145 / 3152130.3152135 (cit. on pp. 4, 23, 30).

[105] J. Haladjian, A. Ermis, Z. Hodaie, and B. Brügge. "iPig: Towards tracking the behavior of free-roaming pigs". In: Proceedings of the Fourth International Conference on Animal-Computer Interaction - ACI2017. New York, New York, USA: ACM Press, 2017, 10:1-10:5. DOI: 10.1145/3152130. 3152145 (cit. on pp. 23, 30).

[106] J. P. Hansen, A. Alapetite, I. S. MacKenzie, and E. Møllenbach. "The use of gaze to control drones". In: Proceedings of the Symposium on Eye Tracking Research and Applications - ETRA '14. New York, New York, USA: ACM Press, 2014, pp. 27-34. DOI: 10.1145/2578153.2578156 (cit. on p. 173). 
[107] D. Haraway. When Species Meet. University of Minnesota Press, 2007, p. 440 (cit. on p. 26).

[108] S. D. E. Held and M. Špinka. "Animal play and animal welfare". In: Animal Behaviour 81.5 (2011), pp. 891-899. DOI: 10.1016/j.anbehav.2011.01.007 (cit. on p. 222).

[109] F. Hemmert et al. "Co-designing with children: a comparison of embodied and disembodied sketching techniques in the design of child age communication devices". In: Proceedings of the 9th International Conference on Interaction Design and Children - IDC '10. New York, New York, USA: ACM Press, 2010, pp. 202-205. DOI: 10.1145/1810543.1810571 (cit. on p. 199).

[110] O. A. B. Henkemans et al. "Design and evaluation of a personal robot playing a self-management education game with children with diabetes type 1". In: International Journal of Human-Computer Studies 106.January (2017), pp. 63-76. DOI: 10.1016/j.ijhcs.2017.06.001 (cit. on p. 170).

[111] N. F. H. Hermans and J. H. Eggen. "Beyond Barriers: Exploring Opportunities of Digital Technology to Encourage Personal Interaction between Captive Orangutans and Zoo Visitors". In: HCI Goes to the Zoo, CHI 2016 Workshops. 2016, pp. 1-7 (cit. on pp. 23, 24, 155, 159, 226).

[112] A. R. Hevner, S. T. March, J. Park, and S. Ram. "Design Science in Information Systems Research". In: Design Science in IS Research MIS Quarterly 28.1 (2004), pp. 75-105. DOI: 10.2307/25148625. eprint: /dl.acm.org/ citation.cfm?id=2017212.2017217 (http:) (cit. on p. 6).

[113] I. Hirskyj-Douglas, H. Luo, and J. C. Read. "Is My Dog Watching TV?" In: NordiCHI'14 - Workshop on Animal-Computer Interaction (ACI): Pushing Boundaries beyond 'Human'. 2014 (cit. on pp. 5, 23, 38, 96, 118).

[114] I. Hirskyj-Douglas and J. C. Read. "DoggyVision: Examining how Dogs (Canis Lupus Familiaris) Interact with Media Using a Dog Driven Proximity Tracker Device". In: Animal Behavior and Cognition (submitted) (2018) (cit. on pp. 22, 23, 36, 38, 43).

[115] I. Hirskyj-Douglas and J. C. Read. "The Ethics of How to Work with Dogs in Animal Computer Interaction". In: Measuring Behavior 2016. 2016, pp. 459-464 (cit. on pp. 43, 44). 
[116] I. Hirskyj-Douglas and J. C. Read. "Who Is Really In The Center Of Dog Computer Design?" In: Proceedings of the 2014 Workshops on Advances in Computer Entertainment Conference - ACE '14 Workshops. New York, New York, USA: ACM Press, 2014, 2:1-2:5. DOI: 10.1145/2693787.2693793 (cit. on pp. 26, 42, 55).

[117] I. Hirskyj-douglas, J. C. Read, and M. Horton. "Animal Personas : Representing Dog Stakeholders in Interaction Design". In: British HCI 2017. 2016, pp. 1-13 (cit. on pp. 35, 200, 225).

[118] I. Hirskyj-Douglas, J. Read, and B. Cassidy. "A dog centred approach to the analysis of dogs' interactions with media on TV screens". In: International Journal of Human-Computer Studies 98 (2017), pp. 208-220. DOI: 10.1016/ j.ijhcs.2016.05.007 (cit. on pp. 19, 23, 33, 36, 38, 42, 43).

[119] I. Hirskyj-Douglas, P. Pons, J. Read, and J. Jaen. "Seven Years after the Manifesto: Literature Review and Research Directions for Technologies in Animal Computer Interaction". In: Multimodal Technologies and Interaction 2.2 (2018), p. 30. DOI: 10.3390/mti2020030 (cit. on p. 10).

[120] I. Hirskyj-Douglas et al. "Where HCI meets ACI". In: Proceedings of the 9th Nordic Conference on Human-Computer Interaction - NordiCHI '16. New York, New York, USA: ACM Press, 2016, pp. 1-3. DOI: 10.1145/2971485. 2987675 (cit. on p. 41).

[121] M. Hodgenboom. The traits that make human beings unique. BBC (cit. on p. 18).

[122] A. Hodgson, N. Kelly, and D. Peel. "Unmanned aerial vehicles (UAVs) for surveying Marine Fauna: A dugong case study". In: PLoS ONE 8.11 (2013), pp. 1-15. DOI: 10.1371/journal.pone.0079556 (cit. on pp. 90, 139).

[123] M. S. Horn, R. J. Crouser, and M. U. Bers. "Tangible interaction and learning: the case for a hybrid approach". In: Personal and Ubiquitous Computing 16.4 (2011), pp. 379-389. DOI: 10.1007/s00779-011-0404-2 (cit. on p. 169).

[124] J. P. Hourcade. Child computer interaction. CreateSpace Independent Publishing Platform, 2015, p. 296 (cit. on p. 169).

[125] S. Howell et al. "A stereo music system as environmental enrichment for captive chimpanzees." In: Lab animal 32.10 (2003), pp. 31-36. DOI: 10 . 1038/laban1103-31 (cit. on p. 155). 
[126] J. Höysniemi, P. Hämäläinen, and L. Turkki. "Wizard of Oz prototyping of computer vision based action games for children". In: Proceeding of the 2004 conference on Interaction design and children building a community - IDC '04. New York, New York, USA: ACM Press, 2004, pp. 27-34. DoI: 10.1145/1017833.1017837 (cit. on pp. 171, 199).

[127] J. Höysniemi, P. Hämäläinen, L. Turkki, and T. Rouvi. "Children's intuitive gestures in vision-based action games". In: Communications of the ACM 48.1 (2005), pp. 44-50. DOI: 10.1145/1039539.1039568 (cit. on pp. 171, 222, $254)$.

[128] H.-S. Hsiao and J.-C. Chen. "Using a gesture interactive game-based learning approach to improve preschool children's learning performance and motor skills". In: Computers \&f Education 95 (2016), pp. 151-162. DOI: 10.1016/j.compedu.2016.01.005 (cit. on p. 170).

[129] F. Hu, D. Silver, and A. Trude. "LonelyDog@Home". In: 2007 IEEE/WIC/ACM International Conferences on Web Intelligence and Intelligent Agent Technology - Workshops. IEEE, 2007, pp. 333-337. DOI: 10.1109/WI-IATW. 2007.74 (cit. on pp. 22, 23, 56, 63, 225, 254).

[130] G. Hu et al. "DT-DT: Top-down Human Activity Analysis for Interactive Surface Applications". In: Proceedings of the Ninth ACM International Conference on Interactive Tabletops and Surfaces - ITS '14. New York, New York, USA: ACM Press, 2014, pp. 167-176. DOI: 10.1145/2669485.2669501 (cit. on pp. 86, 124).

[131] J. Huizinga. Homo ludens. Groningen, The Nederlands: Wolters-Noordhoff, 1985 (cit. on pp. 48, 96, 198, 222).

[132] M. M. Jackson et al. "FIDO - Facilitating interactions for dogs with occupations: wearable communication interfaces for working dogs". In: Personal and Ubiquitous Computing 19.1 (2015), pp. 155-173. DOI: 10.1007/s00779014-0817-9 (cit. on pp. 23, 29, 121).

[133] M. M. Jackson et al. "FIDO - facilitating interactions for dogs with occupations: Wearable Dog-Activated Interfaces". In: Proceedings of the 17th annual international symposium on International symposium on wearable computers - ISWC '13. New York, New York, USA: ACM Press, 2013, pp. 81-88. DOI: 10.1145/2493988.2494334 (cit. on pp. 4, 23, 29, 121).

[134] R. J. Jacob and K. S. Karn. "Eye Tracking in Human-Computer Interaction and Usability Research: Ready to Deliver the Promises". In: The Mind's 
Eye. Elsevier, 2003, pp. 573-605. DOI: 10.1016/B978-044451020-4/50031-1 (cit. on p. 37).

[135] S. Jeong et al. "A Social Robot to Mitigate Stress, Anxiety, and Pain in Hospital Pediatric Care". In: Proceedings of the Tenth Annual ACM/IEEE International Conference on Human-Robot Interaction Extended Abstracts - HRI'15 Extended Abstracts. 2015, pp. 103-104. DOI: 10.1145/2701973. 2702028 (cit. on p. 224).

[136] R. A. Johnson, R. L. Meadows, J. S. Haubner, and K. Sevedge. "Animalassisted activity among patients with cancer: effects on mood, fatigue, selfperceived health, and sense of coherence". In: Oncology nursing forum 35.2 (2008), pp. 225-232. DOI: 10.1188/08.ONF.225-232 (cit. on p. 223).

[137] O. Johnston-Wilder et al. "Sensing the shape of canine responses to cancer". In: Proceedings of the 12th International Conference on Advances in Computer Entertainment Technology - ACE '15. New York, New York, USA: ACM Press, 2015, 63:1-63:4. DoI: 10.1145/2832932.2837017 (cit. on pp. 21, $23,31,32,42)$.

[138] T. Jokela, P. P. Rezaei, and K. Väänänen. "Using Elicitation Studies to Generate Collocated Interaction Methods". In: Proceedings of the 18th International Conference on Human-Computer Interaction with Mobile Devices and Services Adjunct (2016), pp. 1129-1133. DOI: 10.1145/2957265. 2962654 (cit. on p. 172).

[139] B. Jones, H. Benko, E. Ofek, and A. D. Wilson. "IllumiRoom: Peripheral Projected Illusions for Interactive Experiences". In: Proceedings of the SIGCHI Conference on Human Factors in Computing Systems - CHI '13. 2013, pp. 869-878. DOI: $10.1145 / 2470654.2466112$ (cit. on pp. $82,124,250$, $254)$.

[140] B. Jones et al. "RoomAlive: magical experiences enabled by scalable, adaptive projector-camera units". In: Proceedings of the 27th annual ACM symposium on User interface software and technology - UIST '14. 2014, pp. 637644. DOI: $10.1145 / 2642918.2647383$ (cit. on pp. 82, 124, 168, 250).

[141] M. K. Jones. Human-computer interaction: A design guide. Educational Technology, 1989 (cit. on p. 18).

[142] I. K. H. Jørgensen and H. Wirman. "Multispecies methods, technologies for play". In: Digital Creativity 6268.March (2016), pp. 1-15. DoI: 10.1080/ 14626268.2016.1144617 (cit. on pp. 4, 99, 156, 159, 198, 200, 222, 226, 233). 
[143] A. Jukan, X. Masip-Bruin, and N. Amla. "Smart Computing and Sensing Technologies for Animal Welfare: A Systematic Review". In: $A C M$ Computing Surveys 50.1 (2017), pp. 1-27. DOI: 10.1145/3041960. arXiv: $1609.00627 \mathrm{v} 1$ (cit. on pp. 4, 17, 21, 22, 46).

[144] S. Jurdi et al. "A systematic review of game technologies for pediatric patients". In: Computers in Biology and Medicine 97.April (2018), pp. 89112. DOI: 10.1016/j.compbiomed.2018.04.019 (cit. on p. 224).

[145] M. Kaminski, T. Pellino, and J. Wish. "Play and pets: The physical and emotional impact of child-life and pet therapy on hospitalized children". In: Children's Health Care 31.4 (2002), pp. 321-335. DOI: 10.1207/S15326888CHC3104 5 (cit. on p. 224).

[146] H. Kamioka et al. "Effectiveness of animal-assisted therapy: A systematic review of randomized controlled trials". In: Complementary therapies in medicine 22.2 (2014), pp. 371-390. DOI: 10.1016/j.ctim.2013.12.016 (cit. on p. 70).

[147] M. Karam and m. c. Schraefel. A Taxonomy of Gestures in Human Computer Interactions. 2005. DOI: 10.1.1.97.5474 (cit. on p. 179).

[148] J. Karlsson, K. Ren, and H. Li. "Tracking and Identification of Animals for a Digital Zoo". In: 2010 IEEE/ACM Int'l Conference on Green Computing and Communications \& Int'l Conference on Cyber, Physical and Social Computing. IEEE, 2010, pp. 510-515. DoI: 10.1109/GreenComCPSCom.2010.69 (cit. on pp. 100, 122).

[149] A. Kerepesi, A. Dóka, and Á. Miklósi. "Dogs and their human companions: The effect of familiarity on dog-human interactions". In: Behavioural Processes 110 (2015), pp. 27-36. DOI: 10.1016/j.beproc.2014.02.005 (cit. on pp. 23,36$)$.

[150] R. Kimura et al. "Trial of Robot assisted activity using robotic pets in children hospital". In: Proceedings of the SICE Annual Conference. 2004, pp. 2615-2620 (cit. on p. 224).

[151] F. Kistler and E. André. "User-defined body gestures for an interactive storytelling scenario". In: Lecture Notes in Computer Science (including subseries Lecture Notes in Artificial Intelligence and Lecture Notes in Bioinformatics) 8118 LNCS.PART 2 (2013), pp. 264-281. DOI: 10.1007/978-3642-40480-1_17 (cit. on p. 172). 
[152] H. Kobayashi and H. Kudo. "Carrier Pigeon-like Sensing System: Beyond Human-Red Forest Interactions". In: Proceeding of Balance-Unbalance International Conference. 2013, pp. 1-15 (cit. on p. 44).

[153] H. Kobayashi et al. "Playful rocksalt system: animal-computer interaction design in wild environments". In: Proceedings of the 12th International Conference on Advances in Computer Entertainment Technology $A C E$ '15. New York, New York, USA: ACM Press, 2015, pp. 1-4. DOI: $10.1145 / 2832932.2837012$ (cit. on pp. 23, 31).

[154] K. R. Konda, A. Königs, H. Schulz, and D. Schulz. "Real time interaction with mobile robots using hand gestures". In: Proceedings of the seventh annual ACM/IEEE international conference on Human-Robot Interaction - HRI '12. New York, New York, USA: ACM Press, 2012, pp. 177-178. DOI: 10.1145/2157689.2157743 (cit. on p. 173).

[155] C. Kray, D. Nesbitt, J. Dawson, and M. Rohs. "User-defined gestures for connecting mobile phones, public displays, and tabletops". In: Proceedings of the 12th international conference on Human computer interaction with mobile devices and services - MobileHCI '10. New York, New York, USA: ACM Press, 2010, pp. 239-248. DOI: 10.1145/1851600.1851640 (cit. on pp. 168, 172).

[156] B. L. Krebs and J. V. Watters. "Using Technology Driven Environments to Promote Animal Well-Being in Zoos". In: HCI Goes to the Zoo, CHI 2016 Workshops. 2016, pp. 1-5 (cit. on pp. 23, 25).

[157] E. Kurdyukova, M. Redlin, and E. André. "Studying user-defined iPad gestures for interaction in multi-display environment". In: Proceedings of the 2012 ACM international conference on Intelligent User Interfaces IUI '12. New York, New York, USA: ACM Press, 2012, pp. 93-96. DOI: $10.1145 / 2166966.2166984$ (cit. on p. 172).

[158] C. Ladha et al. "Dog's Life: Wearable Activity Recognition for Dogs Cassim". In: Proceedings of the 2013 ACM international joint conference on Pervasive and ubiquitous computing - UbiComp '13. New York, New York, USA: ACM Press, 2013, pp. 415-418. DOI: 10.1145/2493432.2493519 (cit. on pp. $23,28,81,99,121,123)$.

[159] V. Lambert, J. Coad, P. Hicks, and M. Glacken. "Social spaces for young children in hospital". In: Child: Care, Health and Development 40.2 (2014), pp. 195-204. DOI: 10.1111/cch.12016 (cit. on pp. 232, 241). 
[160] S. Lawson, B. Kirman, and C. Linehan. "Power, participation, and the dog internet". In: Interactions 23.4 (2016), pp. 37-41. DOI: 10.1145/2942442 (cit. on pp. 22, 23, 31, 32, 44).

[161] S. Lawson et al. "Problematising Upstream Technology through Speculative Design: The Case of Quantified Cats and Dogs". In: Proceedings of the 33rd Annual ACM Conference on Human Factors in Computing Systems - CHI '15. New York, New York, USA: ACM Press, 2015, pp. 2663-2672. DOI: 10.1145/2702123.2702260 (cit. on p. 35).

[162] M. C. Leach et al. "The Assessment of Post-Vasectomy Pain in Mice Using Behaviour and the Mouse Grimace Scale". In: PLoS ONE 7.4 (2012), e35656. DOI: 10.1371/journal.pone.0035656 (cit. on p. 37).

[163] S.-S. Lee et al. "Towards more natural digital content manipulation via user freehand gestural interaction in a living room". In: Proceedings of the 2013 ACM international joint conference on Pervasive and ubiquitous computing - UbiComp '13. New York, New York, USA: ACM Press, 2013, p. 617. DoI: 10.1145/2493432.2493480 (cit. on p. 172).

[164] S. P. Lee et al. "A mobile pet wearable computer and mixed reality system for human-poultry interaction through the internet". In: Personal and Ubiquitous Computing 10.5 (2006), pp. 301-317. DOI: 10.1007/s00779-0050051-6 (cit. on pp. 16, 21, 23, 27, 39, 43, 53, 81, 100, 122).

[165] G. Lemasson, D. Duhaut, and S. Pesty. "Dog: Can you feel it?" In: Animal Computer Interaction @ British Human Computer Interaction (BHCI). 2015, pp. 7-9 (cit. on pp. 21, 23, 27, 29).

[166] K. Leo and B. Tan. "User-tracking mobile floor projection virtual reality game system for paediatric gait \& dynamic balance training". In: Proceedings of the 4th International Convention on Rehabilitation Engineering \&6 Assistive Technology. 2010, 25:1-25:4 (cit. on pp. 70, 222).

[167] C. Li, D. Goldgof, and L. Hall. "Knowledge-based classification and tissue labeling of MR images of human brain". In: IEEE Transactions on Medical Imaging 12.4 (1993), pp. 740-750. DOI: 10.1109/42.251125 (cit. on pp. 113, 131).

[168] S.-C. Lin, A.-S. Liu, T.-W. Hsu, and L.-C. Fu. "Representative Body Points on Top-View Depth Sequences for Daily Activity Recognition". In: 2015 IEEE International Conference on Systems, Man, and Cybernetics. 2015, pp. 2968-2973. DOI: 10.1109/SMC.2015.516 (cit. on p. 124). 
[169] S. W. Line, H. Markowitz, K. N. Morgan, and S. Strong. "Effects of cage size and environmental enrichment on behavioral and physiological responses of rhesus macaques to the stress of daily events". In: Through the looking glass issues of psychological wellbeing in captive nonhuman primates. Ed. by M. A. Novak and A. J. Petto. 1991, pp. 160-179. Dor: 10.1037/10080-016 (cit. on p. 155).

[170] K. Lockhart. Chimpanzee bats drone out of mid-air. 2015 (cit. on p. 3).

[171] J. Majikes et al. "Balancing Noise Sensitivity, Response Latency, and Posture Accuracy for a Computer-Assisted Canine Posture Training System". In: International Journal of Human-Computer Studies 98 (2017), pp. 179 195. DOI: 10.1016/j.ijhcs.2016.04.010 (cit. on pp. 21-23, 29, 30).

[172] L. Malinverni, J. Mora-Guiard, and N. Pares. "Towards methods for evaluating and communicating participatory design: A multimodal approach". In: International Journal of Human-Computer Studies 94 (2016), pp. 5363. DOI: 10.1016/j.ijhcs.2016.03.004 (cit. on p. 171).

[173] C. Mancini. "Animal-computer interaction: a manifesto". In: Magazine interactions 18.4 (2011), pp. 69-73. DOI: 10.1145/1978822.1978836 (cit. on pp. $3,4,10,16,17,21,37,41-43,50,80,96,118,154,156,198,222)$.

[174] C. Mancini. "Animal-computer interaction (ACI): changing perspective on HCI, participation and sustainability". In: CHI '13 Extended Abstracts on Human Factors in Computing Systems. New York, New York, USA: ACM Press, 2013, pp. 2227-2236. DOI: 10.1145/2468356.2468744 (cit. on pp. 4 , $21,41,50,80,96,118,154,222)$.

[175] C. Mancini. "Towards an Animal-Centred Ethics for Animal-Computer Interaction". In: International Journal of Human-Computer Studies 98 (2017), pp. 221-233. DOI: 10.1016/j.ijhcs.2016.04.008 (cit. on pp. 18, 19, 43, 104).

[176] C. Mancini and J. van der Linden. "UbiComp for animal welfare: envisioning smart environments for kenneled dogs". In: Proceedings of the 2014 ACM International Joint Conference on Pervasive and Ubiquitous Computing. 2014, pp. 117-128. DOI: 10.1145/2632048.2632073 (cit. on pp. 4, 16, $17,68)$.

[177] C. Mancini et al. "Animal-computer interaction (ACI): pushing boundaries beyond 'human'". In: Proceedings of the 8th Nordic Conference on HumanComputer Interaction: Fun, Fast, Foundational. 2014, pp. 833-836. DOI: 10.1145/2639189.2654841 (cit. on pp. 16, 198). 
[178] C. Mancini et al. "Animal-computer interaction SIG". In: Proceedings of the 2012 ACM annual conference extended abstracts on Human Factors in Computing Systems Extended Abstracts. 2012, pp. 1233-1236. DOI: 10.1145/ 2212776.2212431 (cit. on pp. 17, 52, 222).

[179] C. Mancini, J. van der Linden, J. Bryan, and A. Stuart. "Exploring interspecies sensemaking: Dog Tracking Semiotics and Multispecies Ethnography". In: Proceedings of the 2012 ACM Conference on Ubiquitous Computing - UbiComp '12. New York, New York, USA: ACM Press, 2012, pp. 143152. DOI: $10.1145 / 2370216.2370239$ (cit. on pp. $23,28,81,120,123$ ).

[180] C. Mancini, R. Harris, B. Aengenheister, and C. Guest. "Re-Centering Multispecies Practices: A Canine Interface for Cancer Detection Dogs". In: Proceedings of the 33rd Annual ACM Conference on Human Factors in Computing Systems - CHI '15. New York, New York, USA: ACM Press, 2015, pp. 2673-2682. DOI: 10.1145/2702123.2702562 (cit. on pp. 4, 21, 23, $31,42,54)$.

[181] C. Mancini et al. "Towards multispecies interaction environments: extending accessibility to canine users". In: Proceedings of the Third International Conference on Animal-Computer Interaction - ACI '16. New York, New York, USA: ACM Press, 2016, pp. 1-10. DOI: 10.1145/2995257.2995395 (cit. on pp. 18, 23-25, 42).

[182] D. Mankoff, A. K. Dey, J. Mankoff, and K. Mankoff. "Supporting Interspecies Social Awareness: Using peripheral displays for distributed pack awareness". In: Proceedings of the 18th annual ACM symposium on User interface software and technology. 2005, pp. 253-258. DOI: 10.1145/1095034. 1095076 (cit. on pp. 56, 63).

[183] S. Mann. The Encyclopedia of Human-Computer Interaction, 2nd Ed. Wearable Computing (cit. on p. 27).

[184] J. Marco et al. "Bringing Tabletop Technologies to Kindergarten Children". In: Proceedings of the 23rd British HCI Group Annual Conference on People and Computers: Celebrating People and Technology. 2009, pp. 103-111 (cit. on p. 168).

[185] C. F. Martin and R. W. Shumaker. "Great Ape Touch-Panel Tasks: A Platform for Research, Enrichment, and Conservation". In: HCI Goes to the Zoo, CHI 2016 Workshops. 2016, pp. 1-5 (cit. on pp. 23, 34, 35). 
[186] C. Maternaghan and K. J. Turner. "A configurable telecare system". In: Proceedings of the 4th International Conference on Pervasive Technologies Related to Assistive Environments - PETRA '11. New York, New York, USA: ACM Press, 2011, 14:1-14:8. DOI: 10.1145/2141622.2141639 (cit. on p. 68).

[187] T. Matsuzawa. "The Ai project: historical and ecological contexts". In: Animal cognition 6.4 (2003), pp. 199-211. DOI: 10.1007/s10071-003-0199-2 (cit. on p. 52).

[188] J. McDermott and M. Hauser. "Are consonant intervals music to their ears? Spontaneous acoustic preferences in a nonhuman primate". In: Cognition 94.2 (2004), pp. 11-21. DOI: 10.1016/j.cognition. 2004.04 .004 (cit. on pp. $154,155,160)$.

[189] R. E. McGrath. "Species-appropriate computer mediated interaction". In: Proceedings of the 27 th international conference extended abstracts on $\mathrm{Hu}$ man factors in computing systems - CHI EA '09 (2009), pp. 2529-2534. DOI: $10.1145 / 1520340.1520357$ (cit. on pp. $3,4,60$ ).

[190] S. Mealin, I. X. Domínguez, and D. L. Roberts. "Semi-supervised classification of static canine postures using the Microsoft Kinect". In: Proceedings of the Third International Conference on Animal-Computer Interaction $A C I$ '16. New York, New York, USA: ACM Press, 2016, pp. 1-4. DOI: $10.1145 / 2995257.3012024$ (cit. on pp. 23, 37-40, 236, 251, 253).

[191] S. Mealin, S. Howell, and D. L. Roberts. "Towards Unsupervised Canine Posture Classification via Depth Shadow Detection and Infrared Reconstruction for Improved Image Segmentation Accuracy". In: Conference on Biomimetic and Biohybrid Systems. Ed. by N. F. Lepora et al. Vol. 9793. Lecture Notes in Computer Science. Springer International Publishing, 2016, pp. 155-166. DOI: 10.1007/978-3-319-42417-0_15 (cit. on pp. 251, 253).

[192] S. Mealin et al. "Creating an Evaluation System for Future Guide Dogs: A Case Study of Designing for Both Human and Canine Needs". In: Proceedings of the Fourth International Conference on Animal-Computer Interaction - ACI2017. New York, New York, USA: ACM Press, 2017, pp. 1-6. DOI: $10.1145 / 3152130.3152148$ (cit. on pp. 23, 28).

[193] S. Mealin et al. "Towards the non-visual monitoring of canine physiology in real-time by blind handlers". In: Proceedings of the 12th International Conference on Advances in Computer Entertainment Technology - ACE 
'15. New York, New York, USA: ACM Press, 2015, pp. 1-8. DOI: 10.1145/ 2832932.2837018 (cit. on pp. 4, 21, 23, 28).

[194] F. Michaud and S. Caron. "Roball, the Rolling Robot". In: Autonomous Robots 12.2 (2002), pp. 211-222. DOI: 10.1023/A:1014005728519 (cit. on p. 170).

[195] M. Micire et al. "Analysis of natural gestures for controlling robot teams on multi-touch tabletop surfaces". In: Proceedings of the ACM International Conference on Interactive Tabletops and Surfaces - ITS '09. New York, New York, USA: ACM Press, 2009, pp. 41-48. DOI: 10.1145/1731903.1731912 (cit. on p. 168).

[196] Á. Miklósi et al. "A Simple Reason for a Big Difference". In: Current Biology 13.9 (2003), pp. 763-766. DOI: 10.1016/S0960-9822(03)00263-X (cit. on p. 5).

[197] P. E. Miller. "Vision in Animals - What do Dogs and Cats See?" In: The 25th Annual Waltham/OSU Symposium. Small Animal Ophthalmology. 2001 (cit. on p. 96).

[198] D. S. Mills, ed. The Encyclopedia of Applied Animal Behaviour and Welfare. CABI International, Wallingford, 2010, p. 685 (cit. on p. 97).

[199] J. A. Mocholí, J. Jaén, and A. Catalá. "A Model of Affective Entities for Effective Learning Environments". In: Innovations in Hybrid Intelligent Systems. 2007, pp. 337-344. DOI: 10.1007/978-3-540-74972-1_44 (cit. on p. 71).

[200] J. Mora-Guiard, C. Crowell, N. Pares, and P. Heaton. "Lands of Fog: Helping Children with Autism in Social Interaction through a Full-Body Interactive Experience". In: Proceedings of the The 15th International Conference on Interaction Design and Children - IDC '16. New York, New York, USA: ACM Press, 2016, pp. 262-274. DOI: 10.1145/2930674.2930695 (cit. on pp. 168, 170).

[201] A. Moreno et al. "Augmenting traditional playground games to enhance game experience". In: 7th International Conference on Intelligent Technologies for Interactive Entertainment (INTETAIN). Vol. 17. 2015, pp. 140149. DOI: 10.4108/icst.intetain.2015.259399 (cit. on p. 124).

[202] M. R. Morris. "Web on the Wall: Insights from a Multimodal Interaction Elicitation Study". In: Proceedings of the 2012 ACM international confer- 
ence on Interactive tabletops and surfaces. ACM Press, 2012, pp. 95-104. DOI: $10.1145 / 2396636.2396651$ (cit. on pp. 168, 172).

[203] M. R. Morris, J. O. Wobbrock, and A. D. Wilson. "Understanding users' preferences for surface gestures". In: Proceedings of Graphics Interface 2010. 2010, pp. 261-268 (cit. on p. 168).

[204] A. Morrison, R. H. Møller, C. Manresa-Yee, and N. Eshraghi. "The impact of training approaches on experimental setup and design of wearable vibrotactiles for hunting dogs". In: Proceedings of the Third International Conference on Animal-Computer Interaction - ACI '16. New York, New York, USA: ACM Press, 2016, pp. 1-10. DOI: 10.1145/2995257.2995391 (cit. on pp. 16, 21, 23, 27, 29).

[205] C. Mühlenbeck, K. Liebal, C. Pritsch, and T. Jacobsen. "Gaze Duration Biases for Colours in Combination with Dissonant and Consonant Sounds: A Comparative Eye-Tracking Study with Orangutans". In: PLOS ONE 10.10 (2015). Ed. by M. S. Malmierca, e0139894. DOI: 10.1371/journal. pone.0139894 (cit. on p. 160).

[206] C. Müller et al., eds. Body - Language - Communication: An International Handbook on Multimodality in Human Interaction. Gruyter Mouton, 2013 (cit. on p. 5).

[207] M. J. Muller. "Participatory design: the third space in HCI". In: The human-computer interaction handbook. Vol. 4235. 2002, pp. 1051-1068. DOI: 10.1145/153571.255960 (cit. on pp. 7, 199).

[208] M. J. Muller and S. Kuhn. "Participatory design". In: Communications of the ACM 36.6 (1993), pp. 24-28. DOI: 10.1145/153571.255960 (cit. on p. 199).

[209] V. Nacher, F. Garcia-Sanjuan, and J. Jaen. "Evaluating the Usability of a Tangible-Mediated Robot for Kindergarten Children Instruction". In: 2016 IEEE 16th International Conference on Advanced Learning Technologies (ICALT). IEEE, 2016, pp. 130-132. DOI: 10.1109/ICALT.2016.59 (cit. on p. 170).

[210] V. Nacher, F. Garcia-Sanjuan, and J. Jaen. "Interactive technologies for preschool game-based instruction: Experiences and future challenges". In: Entertainment Computing 17 (2016), pp. 19-29. DOI: 10.1016/j.entcom. 2016.07.001 (cit. on p. 96). 
[211] V. E. Nahapetyan and V. M. Khachumov. "Gesture recognition in the problem of contactless control of an unmanned aerial vehicle". In: Optoelectronics, Instrumentation and Data Processing 51.2 (2015), pp. 192-197. DOI: 10.3103/S8756699015020132 (cit. on p. 173).

[212] F. K. D. Nahm, A. Perret, D. G. Amaral, and T. D. Albright. "How Do Monkeys Look at Faces?" In: Journal of Cognitive Neuroscience 9.5 (1997), pp. 611-623. DOI: 10.1162/jocn.1997.9.5.611 (cit. on p. 37).

[213] E. Nannoni, G. Martelli, and L. Sardi. "Enrichments For Pigs: Improving animal-environment relations". In: Proceedings of the Fourth International Conference on Animal-Computer Interaction - ACI201\%. New York, New York, USA: ACM Press, 2017, pp. 1-6. DoI: 10.1145/3152130.3152151 (cit. on p. 42$)$.

[214] J. K. Nelson and P. C. Shih. "CompanionViz: mediated platform for gauging canine health and enhancing human-pet interactions". In: International Journal of Human-Computer Studies 98 (2017), pp. 169-178. DOI: http: //dx.doi.org/10.1016/j.ijhcs.2016.04.002 (cit. on p. 96).

[215] P. Nepps, C. Stewart, and S Bruckno. "Animal-assisted therapy: Effects on stress, mood, and pain". In: Journal of Lancaster General Hospital 6.2 (2011), pp. 56-59 (cit. on pp. 165, 223).

[216] A. Nijholt, ed. More Playful User Interfaces. Singapore: Springer, 2015. DOI: $10.1007 / 978-981-287-546-4$ (cit. on p. 198).

[217] A. Nijholt, ed. Playful User Interfaces. Singapore: Springer, 2014. DoI: 10. 1007/978-981-4560-96-2 (cit. on pp. 51, 198).

[218] D. A. Norman. The Design of Everyday Things. 1988 (cit. on pp. 19, 20).

[219] D. A. Norman. The invisible computer. Cambridge, MA, USA: MIT Press, 1998 (cit. on p. 51).

[220] D. A. Norman and S. W. Draper. User Centered System Design: New Perspectives on Human-Computer Interaction. 1986 (cit. on p. 7).

[221] S. North. "Salient features, combined detectors and image flipping: an approach to Haar cascades for recognising horses and other complex, deformable objects". In: Proceedings of the Fourth International Conference on Animal-Computer Interaction - ACI2017. New York, New York, USA: 
ACM Press, 2017, pp. 1-6. DOI: 10.1145/3152130.3152143 (cit. on pp. 23, 37-39).

[222] S. North and C. Mancini. "Frameworks for ACI: Animals as stakeholders in the design process". In: Interactions 23.4 (2016), pp. 34-36. DOI: 10.1145/ 2946043 (cit. on pp. 5, 96).

[223] S. North, C. Hall, A. Roshier, and C. Mancini. "HABIT: Horse Automated Behaviour Identification Tool - A Position Paper". In: Proceedings of the British Human Computer Interaction Conference - Animal Computer Interaction Workshop. 2015, pp. 1-4. DOI: 10.1145/rest-of-DOI (cit. on pp. 23, 37-39, 91, 139).

[224] F. Noz and J. An. "Cat Cat Revolution: An Interspecies Gaming Experience". In: Proceedings of the SIGCHI Conference on Human Factors in Computing Systems. 2011, pp. 2661-2664. DOI: 10.1145/1978942.1979331 (cit. on pp. 3, 4, 57, 63, 98, 118, 224, 226, 254).

[225] M. Obaid et al. "A Framework for User-Defined Body Gestures to Control a Humanoid Robot". In: International Journal of Social Robotics 6.3 (2014), pp. 383-396. DOI: 10.1007/s12369-014-0233-3 (cit. on p. 172).

[226] M. Obaid et al. "How would you gesture navigate a drone?: a user-centered approach to control a drone". In: Proceedings of the 20th International Academic Mindtrek Conference on - AcademicMindtrek '16. New York, New York, USA: ACM Press, 2016, pp. 113-121. DOI: 10.1145/2994310.2994348 (cit. on pp. 168, 172, 194, 254).

[227] M. Obaid et al. "User-Defined Body Gestures for Navigational Control of a Humanoid Robot". In: Lecture Notes in Computer Science (including subseries Lecture Notes in Artificial Intelligence and Lecture Notes in Bioinformatics). Vol. 7621 LNAI. 2012, pp. 367-377. DOI: 10.1007/978-3-64234103-8_37 (cit. on pp. 168, 172, 179).

[228] M. Oczak et al. "Automatic estimation of number of piglets in a pen during farrowing, using image analysis". In: Biosystems Engineering 151 (2016), pp. 81-89. DOI: 10.1016/j.biosystemseng.2016.08.018 (cit. on pp. 23, 39).

[229] J. J. Ogden, D. G. Lindburg, and T. L. Maple. "A preliminary study of the effects of ecologically relevant sounds on the behaviour of captive lowland gorillas". In: Applied Animal Behaviour Science 39.2 (1994), pp. 163-176 (cit. on p. 159). 
[230] N. Ohta, H. Nishino, A. Takashima, and A. D. Cheok. "Animal-Human Digital Interface : Can Animals Collaborate with Artificial Presences ?" In: Measuring Behavior 2016. 2016, pp. 455-458 (cit. on pp. 23, 36).

[231] Orangutan using tool. https://www.youtube.com/watch?v=pXOTAgq-eiY. [Online; accessed 16-April-2018]. 2013 (cit. on p. 148).

[232] P. Paci, C. Mancini, and B. A. Price. "Designing for wearability in animal biotelemetry". In: Proceedings of the Third International Conference on Animal-Computer Interaction - ACI '16. New York, New York, USA: ACM Press, 2016, pp. 1-4. DOI: 10.1145/2995257.3012018 (cit. on pp. 22, 23, 28, 250).

[233] P. Paci, C. Mancini, and B. A. Price. "The Role of Ethological Observation for Measuring Animal Reactions to Biotelemetry Devices". In: Proceedings of the Fourth International Conference on Animal-Computer Interaction ACI2017. New York, New York, USA: ACM Press, 2017, pp. 1-12. DOI: $10.1145 / 3152130.3152144$ (cit. on pp. 6, 23, 28).

[234] P. Paci, C. Mancini, and B. A. Price. "Towards a Wearer-Centred Framework for Animal Biotelemetry Designing for wearability". In: Measuring Behavior 2016. 2016, pp. 465-469 (cit. on p. 22).

[235] M. Paldanius et al. "Communication technology for human-dog interaction: exploration of dog owners' experiences and expectations". In: Proceedings of the SIGCHI Conference on Human Factors in Computing Systems. New York, New York, USA: ACM Press, 2011, pp. 2641-2650. DOI: 10.1145/ 1978942.1979329 (cit. on pp. 23, 28, 54, 81, 120, 123).

[236] N. Pares et al. "Promotion of creative activity in children with severe autism through visuals in an interactive multisensory environment". In: Proceeding of the 2005 conference on Interaction design and children - IDC '05. New York, New York, USA: ACM Press, 2005, pp. 110-116. DOI: 10.1145/ 1109540.1109555 (cit. on p. 170).

[237] S. Parsons. "Learning to work together: Designing a multi-user virtual reality game for social collaboration and perspective-taking for children with autism". In: International Journal of Child-Computer Interaction 6 (2015), pp. 28-38. DOI: 10.1016/j.ijcci.2015.12.002 (cit. on p. 96).

[238] PawTrack. PawTrack. GPS Cat Tracking (cit. on pp. 23, 29). 
[239] A. K. Pederson, J. E. King, and V. I. Landau. "Chimpanzee (Pan troglodytes) personality predicts behavior". In: Journal of Research in Personality 39.5 (2005), pp. 534-549. DOI: 10.1016/j.jrp.2004.07.002 (cit. on p. 100).

[240] B. M. Perdue et al. "Technology at the zoo: the influence of a touchscreen computer on orangutans and zoo visitors." In: Zoo biology 31.1 (2012), pp. 27-39. DOI: 10.1002/zoo.20378 (cit. on pp. 3, 21, 23, 34, 97, 99).

[241] K. Pfeil, S. L. Koh, and J. LaViola. "Exploring 3d gesture metaphors for interaction with unmanned aerial vehicles". In: Proceedings of the 2013 international conference on Intelligent user interfaces - IUI '13 (2013), pp. 257-266. DOI: 10.1145/2449396.2449429 (cit. on p. 173).

[242] J. Piaget. The child and reality: Problems of genetic psychology. New York: Grossman, 1973 (cit. on p. 192).

[243] J. Piaget. The Child's Conception of Space. New York: Norton, 1956 (cit. on pp. 192, 193).

[244] R. W. Picard. Affective Computing. MIT Press, 1997 (cit. on p. 71).

[245] T. Piumsomboon, A. Clark, M. Billinghurst, and A. Cockburn. "Userdefined gestures for augmented reality". In: CHI '13 Extended Abstracts on Human Factors in Computing Systems on - CHI EA '13. ACM Press, 2013, pp. 955-960. DOI: 10.1145/2468356.2468527 (cit. on pp. 168, 172).

[246] P. Pons, A. Carrion, and J. Jaen. "Remote Interspecies Interactions: Improving humans and animals' wellbeing through mobile playful spaces". In: Pervasive and Mobile Computing (), (submitted) (cit. on p. 13).

[247] P. Pons, M. Carter, and J. Jaen. "Sound to your objects: a novel design approach to evaluate orangutans' interest in sound-based stimuli". In: Proceedings of the Third International Conference on Animal-Computer Interaction - ACI '16. New York, New York, USA: ACM Press, 2016, pp. 1-5. DOI: $10.1145 / 2995257.2995383$ (cit. on pp. 12, 23, 24, 99, 138, 198, 222, $225)$.

[248] P. Pons and J. Jaen. "Designing interspecies playful interactions: Studying children perceptions of games with animals". In: Proceedings of the Fourth International Conference on Animal-Computer Interaction (ACI 2017). 2017. DOI: $10.1145 / 3152130.3152139$ (cit. on pp. 13, 225, 226, 233, 242). 
[249] P. Pons and J. Jaen. "Enabling Awareness in Playful Environments for Animals using Body Tracking". In: AWARE Workshop on Awareness Interfaces \& Interactions - IUI 2017. 2017 (cit. on p. 14).

[250] P. Pons and J. Jaen. "Enabling Embodied Interactions in Playful Environments for Animals". In: Third International Conference on Animal Computer Interaction - Doctoral Consortium. 2016, pp. 1-5 (cit. on p. 14).

[251] P. Pons and J. Jaen. "Interactive spaces for children: gesture elicitation for controlling ground mini-robots". In: Journal of Ambient Intelligence and Humanized Computing (), (submitted) (cit. on p. 13).

[252] P. Pons and J. Jaen. "Tangible User Interfaces for Zoo Enrichment". In: HCI Goes to the Zoo, CHI 2016 Workshops. 2016, pp. 1-4 (cit. on pp. 3, $12,138,154,157,159,226)$.

[253] P. Pons and J. Jaen. "Towards the Creation of Interspecies Digital Games: An Observational Study on Cats' Interest in Interactive Technologies". In: Proceedings of the 2016 CHI Conference Extended Abstracts on Human Factors in Computing Systems - CHI EA '16. New York, New York, USA: ACM Press, 2016, pp. 1737-1743. DOI: 10.1145/2851581.2892381 (cit. on pp. 14, 87, 100, 137, 198).

[254] P. Pons, J. Jaen, and A. Catala. "Animal Ludens: Building Intelligent Playful Environments for Animals". In: Proceedings of the 2014 Workshops on Advances in Computer Entertainment Conference - ACE '14 Workshops. New York, New York, USA: ACM Press, 2014, pp. 1-6. DOI: 10.1145/ 2693787.2693794 (cit. on pp. 14, 55, 80, 97, 118, 119, 137, 148, 161, 198, $222,224,225)$.

[255] P. Pons, J. Jaen, and A. Catala. "Assessing machine learning classifiers for the detection of animals' behavior using depth-based tracking". In: Expert Systems with Applications 86 (2017), pp. 235-246. DOI: 10.1016/j.eswa. 2017.05.063 (cit. on pp. 11, 37-39, 220, 227, 236, 243).

[256] P. Pons, J. Jaen, and A. Catala. "Detecting Animals' Body Postures Using Depth-Based Tracking Systems". In: Measuring Behavior 2016. 2016, pp. 450-454 (cit. on p. 14).

[257] P. Pons, J. Jaen, and A. Catala. "Developing a depth-based tracking system for interactive playful environments with animals". In: Proceedings of the 12th International Conference on Advances in Computer Entertainment Technology - ACE '15. New York, New York, USA: ACM Press, 2015, pp. 1- 
8. DOI: $10.1145 / 2832932.2837007$ (cit. on pp. 4, 10, 11, 22, 23, 37-39, 42, $109,124,158,220,225,227,236)$.

[258] P. Pons, J. Jaen, and A. Catala. "Envisioning Future Playful Interactive Environments for Animals". In: More Playful User Interfaces. Ed. by A. Nijholt. Springer, 2015, pp. 121-150. DOI: 10.1007/978-981-287-546-4_6 (cit. on pp. 4, 10, 16, 45, 80, 96, 97, 119, 136, 137, 148, 161, 198, 222, 234).

[259] P. Pons, J. Jaen, and A. Catala. "Intelligent playful environments for animals". In: Proceedings of the XVI International Conference on Human Computer Interaction. Vol. 07-09-Sept. 2015. DOI: 10.1145/2829875.2829879 (cit. on p. 14).

[260] P. Pons, J. Jaen, and A. Catala. "Towards Future Interactive Intelligent Systems for Animals: Study and Recognition of Embodied Interactions". In: Proceedings of the 22nd International Conference on Intelligent User Interfaces - IUI '17. New York, New York, USA: ACM Press, 2017, pp. 389400. DOI: $10.1145 / 3025171.3025175$ (cit. on pp. 11, 23, 26, 41, 42, 87, 124, $137,198,222,225,226,236)$.

[261] A. Poole and L. J. Ball. "Eye Tracking in HCI and Usability Research". In: Encyclopedia of Human Computer Interaction. IGI Global, pp. 211-219. DOI: 10.4018/978-1-59140-562-7.ch034 (cit. on p. 37).

[262] PudPod. PupPod Smart Dog Toys (cit. on p. 41).

[263] D. Pyryeskin, M. Hancock, and J. Hoey. "Comparing elicited gestures to designer-created gestures for selection above a multitouch surface". In: Proceedings of the 2012 ACM international conference on Interactive tabletops and surfaces - ITS '12. New York, New York, USA: ACM Press, 2012, pp. 1-10. DOI: 10.1145/2396636.2396638 (cit. on p. 168).

[264] A. Racca et al. "Discrimination of human and dog faces and inversion responses in domestic dogs (Canis familiaris)". In: Animal Cognition 13.3 (2010), pp. 525-533. DOI: 10.1007/s10071-009-0303-3 (cit. on p. 37).

[265] H. S. Raffle, A. J. Parkes, and H. Ishii. "Topobo: a constructive assembly system with kinetic memory". In: System 6.1 (2004), pp. 647-654. DOI: 10.1145/985692.985774 (cit. on p. 170).

[266] J. C. Read and S. MacFarlane. "Using the fun toolkit and other survey methods to gather opinions in child computer interaction". In: Proceeding of the 2006 conference on Interaction design and children - IDC '06. New 
York, New York, USA: ACM Press, 2006, pp. 81-88. DOI: 10.1145/1139073. 1139096 (cit. on pp. 199, 203, 229).

[267] J. C. Read, S. Macfarlane, and C. Casey. "Endurability, Engagement and Expectations: Measuring Children's Fun". In: Interaction Design and Children. 2002, pp. 189-198 (cit. on p. 177).

[268] J. C. Read and P. Markopoulos. "Child-computer interaction". In: International Journal of Child-Computer Interaction 1.1 (2013), pp. 2-6. DOI: 10.1016/j.ijcci.2012.09.001 (cit. on p. 169).

[269] J. C. Read et al. "Child computer interaction". In: Proceeding of the twentysixth annual CHI conference extended abstracts on Human factors in computing systems - CHI '08. Vol. 2. New York, New York, USA: ACM Press, 2008, pp. 2419-2422. DOI: 10.1145/1358628.1358697 (cit. on p. 169).

[270] S. ur Réhman and H. Li. "Using Vibrotactile Language for Multimodal Human Animals Communication and Interaction". In: Proceedings of the 2014 Workshops on Advances in Computer Entertainment Conference ACE '14 Workshops. New York, New York, USA: ACM Press, 2014, pp. 15. DOI: $10.1145 / 2693787.2693792$ (cit. on pp. 23, 27).

[271] D. Reiss, P. Gabrie, N. Gershenfeld, and V. Cerf. The interspecies internet? An idea in progress. TedX Talk (cit. on p. 44).

[272] D. Reiss and B. McCowan. "Spontaneous vocal mimicry and production by bottlenose dolphins (Tursiops truncatus): evidence for vocal learning". In: Journal of Comparative Psychology 107.3 (1993), pp. 301-312 (cit. on p. 22).

[273] B. Resner. "Rover@Home: Computer mediated remote interaction between humans and dogs". PhD thesis. M. Sc. Thesis, Massachusetts Institute of Technology, 2001 (cit. on pp. 16, 21-23, 53).

[274] C. Ribeiro, A. Ferworn, M. Denko, and J. Tran. "Canine Pose Estimation: A Computing for Public Safety Solution". In: 2009 Canadian Conference on Computer and Robot Vision. IEEE, 2009, pp. 37-44. DOI: 10.1109/CRV. 2009.38 (cit. on pp. 23, 29, 121, 123).

[275] S. E. Ritvo. "Music Preference and Discrimination in Three Sumatran Orangutans (Pongo Abelii)". PhD thesis. 2013 (cit. on pp. 154, 155). 
[276] S. E. Ritvo and R. S. Allison. "Challenges Related to Nonhuman AnimalComputer Interaction: Usability and Liking". In: Proceedings of the 2014 Workshops on Advances in Computer Entertainment Conference - ACE '14 Workshops. New York, New York, USA: ACM Press, 2014, pp. 1-7. DOI: 10.1145/2693787.2693795 (cit. on pp. 21, 23, 24, 33, 36, 50, 155).

[277] B. Robins, K. Dautenhahn, R. Te Boekhorst, and C. L. Nehaniv. "Behaviour delay and robot expressiveness in child-robot interactions: a user study on interaction kinesics". In: Proceedings of the 3rd ACMIEEE international conference on Human robot interaction (2008), pp. 17-24. DOI: 10.1145/1349822.1349826 (cit. on p. 170).

[278] B. Robins and K. Dautenhahn. "Tactile Interactions with a Humanoid Robot: Novel Play Scenario Implementations with Children with Autism". In: International Journal of Social Robotics 6.3 (2014), pp. 397-415. DOI: 10.1007/s12369-014-0228-0 (cit. on p. 170).

[279] C. Robinson. "Designing specialized technology to aid assistance dogs". In: Proceedings of the 2014 ACM International Joint Conference on Pervasive and Ubiquitous Computing Adjunct Publication - UbiComp '14 Adjunct. 2014, pp. 343-348. DOI: 10.1145/2638728.2638842 (cit. on p. 54).

[280] C. Robinson et al. "Canine-Centered Interface Design: Supporting the Work of Diabetes Alert Dogs". In: Proceedings of the SIGCHI Conference on Human Factors in Computing Systems. 2014, pp. 3757-3766. DOI: 10.1145/ 2556288.2557396 (cit. on pp. 21-24, 54, 96).

[281] C. Robinson et al. "Designing an emergency communication system for human and assistance dog partnerships". In: Proceedings of the 2015 ACM International Joint Conference on Pervasive and Ubiquitous Computing UbiComp '15. New York, New York, USA: ACM Press, 2015, pp. 337-347. DOI: $10.1145 / 2750858.2805849$ (cit. on pp. $4,23,24,35,42$ ).

[282] C. Robinson et al. "Empowering assistance dogs: an alarm interface for canine use". In: Intelligent Systems for Animal Welfare. 2014, pp. 1-4 (cit. on pp. $16,21,54)$.

[283] A. P. Rossi, S. Rodriguez, and C. R. Cardoso dos Santos. "A dog using skype". In: Proceedings of the Third International Conference on AnimalComputer Interaction - ACI '16. New York, New York, USA: ACM Press, 2016, pp. 1-4. DOI: 10.1145/2995257.3012019 (cit. on pp. 23, 36). 
[284] J. Ruiz, Y. Li, and E. Lank. "User-defined motion gestures for mobile interaction". In: Proceedings of the 2011 annual conference on Human factors in computing systems - CHI '11. ACM Press, 2011, p. 197. DOI: 10.1145/ 1978942.1978971 (cit. on p. 172).

[285] D. M. Rumbaugh. Language learning by a chimpanzee: the Lana Project. New York: Academic Press, 1977 (cit. on pp. 22, 52).

[286] D. M. Rumbaugh et al. "A computer-controlled language training system for investigating the language skills of young apes". In: Behavior Research Methods $\&$ Instrumentation 5.5 (1973), pp. 385-392 (cit. on p. 52).

[287] K. Rust, M. Malu, L. Anthony, and L. Findlater. "Understanding childdefined gestures and children's mental models for touchscreen tabletop interaction". In: Proceedings of the 2014 conference on Interaction design and children - IDC '14. New York, New York, USA: ACM Press, 2014, pp. 201204. DOI: $10.1145 / 2593968.2610452$ (cit. on pp. 171, 199).

[288] T. Salter, K. Dautenhahn, and R. Te Boekhorst. "Learning about natural human-robot interaction styles". In: Robotics and Autonomous Systems 54.2 (2006), pp. 127-134. DOI: 10.1016/j.robot.2005.09.022 (cit. on pp. 168, 170).

[289] J. Sanghvi et al. "Automatic analysis of affective postures and body motion to detect engagement with a game companion". In: Proceedings of the 6th international conference on Human-robot interaction - HRI '11. New York, New York, USA: ACM Press, 2011, pp. 305-311. DOI: 10.1145/1957656. 1957781 (cit. on p. 170).

[290] A. Sanna, F. Lamberti, G. Paravati, and F. Manuri. "A Kinect-based natural interface for quadrotor control". In: Entertainment Computing 4.3 (2013), pp. 179-186. DOI: 10.1016/j.entcom.2013.01.001 (cit. on p. 173).

[291] E. Sato, T. Yamaguchi, and F. Harashima. "Natural interface using pointing behavior for human-robot gestural interaction". In: IEEE Transactions on Industrial Electronics 54.2 (2007), pp. 1105-1112. DOI: 10.1109/TIE.2007. 892728 (cit. on p. 173).

[292] C. P. van Schaik, E. A. Fox, and A. F. Sitompul. "Manufacture and Use of Tools in Wild Sumatran Orangutans Implications for Human Evolution". In: Naturwissenschaften 83 (1996), pp. 186-188. DOI: 10.1007/BF01143062 (cit. on pp. 148, 156). 
[293] M.-M. Schaper, L. Malinverni, and N. Pares. "Sketching through the body: Child-generated gestures in Full-Body Interaction Design". In: Proceedings of the 14th International Conference on Interaction Design and Children IDC '15. New York, New York, USA: ACM Press, 2015, pp. 255-258. DOI: 10.1145/2771839.2771890 (cit. on p. 171).

[294] M.-M. Schaper and N. Pares. "Making Sense of Body and Space through Full-Body Interaction Design". In: Proceedings of the The 15th International Conference on Interaction Design and Children - IDC '16. New York, New York, USA: ACM Press, 2016, pp. 613-618. DOI: 10.1145/2930674. 2935992 (cit. on p. 171).

[295] S. Schwartz. "Separation anxiety syndrome in cats: 136 cases (1991-2000)". In: Journal of the American Veterinary Medical Association 220.7 (2002), pp. 1028-1033. DOI: 10.2460/javma.2002.220.1028 (cit. on p. 68).

[296] S. Schwartz. "Separation anxiety syndrome in dogs and cats". In: Journal of the American Veterinary Medical Association 222.11 (2003), pp. 15261532 (cit. on p. 68).

[297] K. Schweller. "Apes with apps". In: IEEE Spectrum 49.7 (2012), pp. 38-45. DOI: 10.1109/MSPEC.2012.6221081 (cit. on p. 96).

[298] T. Seyed et al. "Eliciting usable gestures for multi-display environments". In: Proceedings of the 2012 ACM international conference on Interactive tabletops and surfaces - ITS '12. New York, New York, USA: ACM Press, 2012, p. 41. DOI: $10.1145 / 2396636.2396643$ (cit. on p. 172).

[299] A. M. Shayan et al. "RoboParrot 2 . 0 : A Multi-Purpose Social Robot". In: IEEE International Conference on Robotics and Mechatronics. 2016, pp. $422-427$ (cit. on p. 224).

[300] S. S. A. Shimon et al. "Exploring User-Defined Back-Of-Device Gestures for Mobile Devices". In: Proceedings of the 17th International Conference on Human-Computer Interaction with Mobile Devices and Services - MobileHCI '15. New York, New York, USA: ACM Press, 2015, pp. 227-232. DOI: $10.1145 / 2785830.2785890$ (cit. on p. 172).

[301] J.-H. Shin, H. Ryu, and S. Jang. "A task-specific interactive game-based virtual reality rehabilitation system for patients with stroke: a usability test and two clinical experiments". In: Journal of NeuroEngineering and Rehabilitation 11.1 (2014), p. 32. DOI: 10.1186/1743-0003-11-32 (cit. on p. 96). 
[302] H. H. Shorey. Animal Communication by Pheromones. New York, New York, USA: Academic Press, 1976, p. 165 (cit. on p. 5).

[303] A. Singh and J. E. Young. "A dog tail for utility robots: Exploring affective properties of tail movement". In: Lecture Notes in Computer Science (including subseries Lecture Notes in Artificial Intelligence and Lecture Notes in Bioinformatics) 8118 LNCS.PART 2 (2013), pp. 403-419. DOI: 10.1007/978-3-642-40480-1_27 (cit. on p. 25).

[304] A. Sipitakiat and N. Nusen. "Robo-blocks: a tangible programming system with debugging for children". In: Proceedings of the 11th International Conference on Interaction Design and Children - IDC '12. April. New York, New York, USA: ACM Press, 2012, p. 98. DOI: 10.1145/2307096.2307108 (cit. on p. 170).

[305] C. T. Snowdon, D. Teie, and M. Savage. "Cats prefer species-appropriate music". In: Applied Animal Behaviour Science 166 (2015), pp. 106-111. DOI: 10.1016/j.applanim.2015.02.012 (cit. on p. 155).

[306] J. Soler-Adillon, J. Ferrer, and N. Pares. "A novel approach to interactive playgrounds: The interactive slide project". In: Proceedings of the 8th International Conference on Interaction Design and Children - IDC '09. New York, New York, USA: ACM Press, 2009, pp. 131-139. DOI: 10.1145/ 1551788.1551811 (cit. on pp. 168, 170, 222).

[307] O. Solomon. "What a Dog Can Do: Children with Autism and Therapy Dogs in Social Interaction". In: Ethos: Journal of the Society for Psychological Anthropology 38.1 (2010), pp. 143-166. DOI: 10.1111/j.15481352.2010.01085.x (cit. on p. 70).

[308] S. Somppi et al. "Dogs do look at images: Eye tracking in canine cognition research". In: Animal Cognition 15.2 (2012), pp. 163-174. DOI: 10.1007/ s10071-011-0442-1 (cit. on pp. 23, 36-38).

[309] M. Sousa et al. "SleeveAR: Augmented Reality for Rehabilitation using Realtime Feedback". In: Proceedings of the 21st International Conference on Intelligent User Interfaces - IUI '16. New York, New York, USA: ACM Press, 2016, pp. 175-185. DOI: 10.1145/2856767.2856773 (cit. on p. 96).

[310] M. Sreelakshmi and T. D. Subash. "Haptic Technology: A comprehensive review on its applications and future prospects". In: Materials Today: Proceedings 4.2 (2017), pp. 4182-4187. DOI: 10.1016/j.matpr.2017.02.120 (cit. on p. 27). 
[311] R. Stiefelhagen et al. "Natural human-robot interaction using speech, head pose and gestures". In: 2004 IEEE/RSJ International Conference on Intelligent Robots and Systems (IROS) (IEEE Cat. No.04CH37566). Vol. 3. IEEE, 2004, pp. 2422-2427. DOI: 10.1109/IROS.2004.1389771 (cit. on p. 173).

[312] K. Subrahmanyam and P. M. Greenfield. "Effect of video game practice on spatial skills in girls and boys". In: Journal of Applied Developmental Psychology 15.1 (1994), pp. 13-32. DOI: 10.1016/0193-3973(94) 90004-3 (cit. on p. 192).

[313] J. Sugiyama, D. Tsetserukou, and J. Miura. "NAVIgoid: Robot navigation with haptic vision". In: SIGGRAPH Asia 2011 Emerging Technologies, $S A$ '11 15.1 (2011), p. 4503. DOI: 10.1145/2073370.2073378 (cit. on p. 173).

[314] K. Svartberg and B. Forkman. "Personality traits in the domestic dog (Canis familiaris)". In: Applied Animal Behaviour Science 79.2 (2002), pp. 133155. DOI: 10.1016/S0168-1591(02)00121-1 (cit. on p. 100).

[315] T. Takahashi, M. Morita, and F. Tanaka. "Evaluation of a tricycle-style teleoperational interface for children: A comparative experiment with a video game controller". In: 2012 IEEE RO-MAN: The 21st IEEE International Symposium on Robot and Human Interactive Communication. IEEE, 2012, pp. 334-338. DOI: 10.1109/ROMAN.2012.6343775 (cit. on p. 170).

[316] R. T.K. C. Tan et al. "Computer Game for Small Pets and Humans". In: Entertainment Computing - ICEC 2007: 6th International Conference. 2007, pp. 28-38. DOI: $10.1007 / 978-3-540-74873-1 \_5$ (cit. on p. 16).

[317] F. Tanaka and T. Takahashi. "A tricycle-style teleoperational interface that remotely controls a robot for classroom children". In: Proceedings of the seventh annual ACM/IEEE international conference on Human-Robot Interaction - HRI '12. New York, New York, USA: ACM Press, 2012, pp. 255256. DOI: $10.1145 / 2157689.2157782$ (cit. on pp. 168, 170).

[318] I. Taranta E.M., T. Simons, R. Sukthankar, and J. Laviola J.J. "Exploring the benefits of context in 3D gesture recognition for game-based virtual environments". In: Transactions on Interactive Intelligent Systems 5.1 (2015). DOI: $10.1145 / 2656345$ (cit. on p. 96).

[319] K. S. Teh, S. P. Lee, and A. D. Cheok. "Poultry.Internet: a remote humanpet interaction system". In: CHI '06 Extended Abstracts on Human Factors 
in Computing Systems. 2006, pp. 251-254. DOI: 10.1145/1125451.1125505 (cit. on p. 53).

[320] L. Tjaden et al. "Children's experiences of dialysis: A systematic review of qualitative studies". In: Archives of Disease in Childhood 97.5 (2012), pp. 395-402. DOI: 10.1136/archdischild-2011-300639 (cit. on p. 241).

[321] D. Todt and M. Naguib. "Vocal Interactions in Birds: The Use of Song as a Model in Communication". In: Advances in the Study of Behavior. 2000, pp. 247-296. DOI: 10.1016/S0065-3454(08)60107-2 (cit. on p. 5).

[322] S. M. Tomecek. Animal Behaviour: Animal Communicaton. New York, New York, USA: Chelsea House, 2009, p. 105 (cit. on p. 5).

[323] R. Trindade et al. "Purrfect Crime". In: Proceedings of the 33rd Annual ACM Conference Extended Abstracts on Human Factors in Computing Systems - CHI EA '15. 2015, pp. 93-96. DOI: 10.1145/2702613.2728660 (cit. on pp. $4,5,21,81,100,123,225,236)$.

[324] H. Väätäjä. "Animal Welfare as a Design Goal in Technology Mediated Human-Animal Interaction". In: Proceedings of the 2014 Workshops on Advances in Computer Entertainment Conference - ACE '14 Workshops. New York, New York, USA: ACM Press, 2014, pp. 1-8. DOI: 10.1145/2693787. 2693797 (cit. on pp. 4, 43, 54).

[325] H. Väätäjä and E. Pesonen. "Ethical issues and guidelines when conducting HCI studies with animals". In: CHI '13 Extended Abstracts on Human Factors in Computing Systems. 2013, pp. 2159-2168. DOI: 10.1145/2468356. 2468736 (cit. on pp. 43, 50, 104, 125).

[326] H. Väätäjä et al. "Technology for Bonding in Human-Animal Interaction". In: Proceedings of the Fourth International Conference on Animal-Computer Interaction (ACI 2017). 2017 (cit. on pp. 225, 233).

[327] V. Vaishnavi, B. Kuechler, and S. Petter. "Design Science Research in Information Systems". In: Design Science Research in Information Systems: (2017), pp. 1-66. DOI: 10.1007/978-3-642-29863-9 (cit. on p. 6).

[328] G. Valentin. "Gestural activity recognition for canine-human communication". In: Proceedings of the 2014 ACM International Symposium on Wearable Computers Adjunct Program - ISWC '14 Adjunct. New York, New York, USA: ACM Press, 2014, pp. 145-149. DOI: 10.1145/2641248.2642733 (cit. on pp. 23, 29, 99, 121, 123). 
[329] G. Valentin et al. "Creating collar-sensed motion gestures for dog-human communication in service applications". In: Proceedings of the 2016 ACM International Symposium on Wearable Computers - ISWC '16. New York, New York, USA: ACM Press, 2016, pp. 100-107. DOI: 10.1145/2971763. 2971788 (cit. on pp. 23, 29, 99).

[330] G. Valentin et al. "Towards a canine-human communication system based on head gestures". In: Proceedings of the 12th International Conference on Advances in Computer Entertainment Technology - ACE '15. New York, New York, USA: ACM Press, 2015, pp. 1-9. DOI: 10.1145/2832932.2837016 (cit. on pp. 21, 23, 29, 121, 123).

[331] R.-D. Vatavu. "Smart-Pockets: Body-deictic gestures for fast access to personal data during ambient interactions". In: International Journal of HumanComputer Studies 103.January (2017), pp. 1-21. DOI: 10.1016/j.ijhcs.2017. 01.005 (cit. on p. 168).

[332] R.-D. Vatavu. "User-defined gestures for free-hand TV control". In: Proceedings of the 10th European conference on Interactive tv and video - EuroiTV '12. New York, New York, USA: ACM Press, 2012, pp. 45-48. DOI: $10.1145 / 2325616.2325626$ (cit. on pp. 168, 172, 254).

[333] R.-D. Vatavu and J. O. Wobbrock. "Between-Subjects Elicitation Studies: Formalization and Tool Support". In: Proceedings of the 2016 CHI Conference on Human Factors in Computing Systems - CHI '16. New York, New York, USA: ACM Press, 2016, pp. 3390-3402. DOI: 10.1145/2858036. 2858228 (cit. on p. 185).

[334] R.-D. Vatavu and J. O. Wobbrock. "Formalizing Agreement Analysis for Elicitation Studies: New Measures, Significance Test, and Toolkit". In: Proceedings of the 33rd Annual ACM Conference on Human Factors in Computing Systems - CHI '15. New York, New York, USA: ACM Press, 2015, pp. 1325-1334. DOI: 10.1145/2702123.2702223 (cit. on p. 185).

[335] E. N. Videan, J. Fritz, S. Howell, and J. Murphy. "Effects of Two Types and Two Genre of Music on Social Behavior in Captive Chimpanzees ( Pan troglodytes )". In: Journal of the American Association for Laboratory Animal Science 46.1 (2007), pp. 66-70 (cit. on p. 155).

[336] J. van Vonderen. Drones with heat-tracking cameras used to monitor koala population. 2015 (cit. on pp. 3, 90, 139, 260). 
[337] D. Voyer, S. Voyer, and M. P. Bryden. "Magnitude of sex differences in spatial abilities: A meta-analysis and consideration of critical variables." In: Psychological Bulletin 117.2 (1995), pp. 250-270. DOI: 10.1037/00332909.117.2.250 (cit. on p. 192).

[338] J. Wainer, B. Robins, F. Amirabdollahian, and K. Dautenhahn. "Using the Humanoid Robot KASPAR to Autonomously Play Triadic Games and Facilitate Collaborative Play Among Children With Autism". In: IEEE Transactions on Autonomous Mental Development 6.3 (2014), pp. 183-199. DOI: 10.1109/TAMD.2014.2303116 (cit. on pp. 168, 170).

[339] L. J. Wallis et al. "Utilising dog-computer interactions to provide mental stimulation in dogs especially during ageing". In: Proceedings of the Fourth International Conference on Animal-Computer Interaction (ACI 2017). 2017, pp. 1-12. DOI: 10.1145/3152130.3152146 (cit. on pp. 23, 36).

[340] S. Watanabe et al. "A new technique for monitoring the detailed behaviour of terrestrial animals: A case study with the domestic cat". In: Applied Animal Behaviour Science 94.1-2 (2005), pp. 117-131. DOI: $10.1016 / \mathrm{j}$. applanim.2005.01.010 (cit. on pp. 23, 29, 122, 123).

[341] S. Webber et al. "HCI Goes to the Zoo". In: Proceedings of the 2016 CHI Conference Extended Abstracts on Human Factors in Computing Systems CHI EA '16. New York, New York, USA: ACM Press, 2016, pp. 3355-3362. DOI: $10.1145 / 2851581.2856485$ (cit. on p. 154).

[342] S. Webber, M. Carter, W. Smith, and F. Vetere. "Interactive Technology and Human-Animal Encounters at the Zoo". In: International Journal of Human-Computer Studies 98 (2017), pp. 150-168. DOI: http://dx.doi.org/ 10.1016/j.ijhcs.2016.05.003 (cit. on pp. 23, 33, 34, 97).

[343] S. Webber et al. "Kinecting with Orangutans: Zoo Visitors' Empathetic Responses to Animals' Use of Interactive Technology". In: Proceedings of the 2017 CHI Conference on Human Factors in Computing Systems - CHI '17. May. New York, New York, USA: ACM Press, 2017, pp. 6075-6088. DOI: $10.1145 / 3025453.3025729$ (cit. on pp. 3-5, 12, 23, 38, 39, 42, 138, 156 , $200,218,222,225,226)$.

[344] A. Weilenmann and O. Juhlin. "Understanding people and animals: the use of a positioning system in ordinary human-canine interaction". In: Proceedings of the 2011 annual conference on Human factors in computing systems - CHI '11. New York, New York, USA: ACM Press, 2011, pp. 2631-2640. DOI: $10.1145 / 1978942.1979328$ (cit. on pp. $23,28,44,54,81,120,123$ ). 
[345] M. Weiser. "The computer for the 21st century". In: Scientific American 265.3 (1991), pp. 94-104 (cit. on pp. 3, 51).

[346] G. M. Weiss, A. Nathan, J. B. Kropp, and J. W. Lockhart. "WagTag: a dog collar accessory for monitoring canine activity levels". In: Proceedings of the 2013 ACM conference on Pervasive and ubiquitous computing adjunct publication. 2013, pp. 405-414. DOI: $10.1145 / 2494091.2495972$ (cit. on pp. $23,28,121,123)$.

[347] D. L. Wells. "Sensory stimulation as environmental enrichment for captive animals: A review". In: Applied Animal Behaviour Science 118.1-2 (2009), pp. 1-11. DOI: 10.1016/j.applanim.2009.01.002 (cit. on p. 97).

[348] F. Wemelsfelder, T. E. Hunter, M. T. Mendl, and A. B. Lawrence. "Assessing the 'whole animal': A free choice profiling approach". In: Animal Behaviour 62.2 (2001), pp. 209-220. DOI: 10.1006/anbe.2001.1741 (cit. on p. 17).

[349] M. Westerlaken and S. Gualeni. "Becoming with: towards the inclusion of animals as participants in design processes". In: Proceedings of the Third International Conference on Animal-Computer Interaction - ACI '16. New York, New York, USA: ACM Press, 2016, pp. 1-10. DOI: 10.1145/2995257. 2995392 (cit. on pp. 23, 26, 41, 42, 198, 222, 225, 226, 233, 256).

[350] M. Westerlaken and S. Gualeni. "Digitally complemented zoomorphism: a theoretical foundation for human-animal interaction design". In: Proceedings of the 6th International Conference on Designing Pleasurable Products and Interfaces - DPPI '13. New York, New York, USA: ACM Press, 2013, pp. 193-200. DOI: 10.1145/2513506.2513527 (cit. on p. 198).

[351] M. Westerlaken and S. Gualeni. "Felino: The Philosophical Practice of Making an Interspecies Videogame". In: The Philosophy of Computer Games Conference. Wirman 2013. 2014, pp. 1-12 (cit. on pp. 4, 5, 16, 17, 23, 33, $34,58,63,96,98,118,222,224,225)$.

[352] M. Westerlaken and S. Gualeni. "Grounded Zoomorphism: An Evaluation Methodology for ACI Design". In: Proceedings of the 2014 Workshops on Advances in Computer Entertainment Conference - ACE '14 Workshops. New York, New York, USA: ACM Press, 2014, pp. 1-6. DOI: 10.1145/ 2693787.2693796 (cit. on pp. 58, 96, 98, 198). 
[353] M. Westerlaken and S. Gualeni. "Situated Knowledges through Game Design : A Transformative Exercise with Ants". In: Proceedings of the Philosophy of Computer Games Conference. 2016 (cit. on pp. 200, 226).

[354] Whistle. Whistle GPS Tracker for Dogs (cit. on pp. 23, 28).

[355] R. Wieringa. "Design science as nested problem solving". In: Proceedings of the 4th International Conference on Design Science Research in Information Systems and Technology - DESRIST 'O9 (2009), p. 1. DOI: 10.1145/ 1555619.1555630 (cit. on p. 7).

[356] Wildlife and Wild Places with Tom Stalf - Orangutan Enrichment. https: //www.youtube.com/watch?v=PZ4GiEiYurE. [Online; accessed 16-April2018]. 2015 (cit. on p. 148).

[357] F. J. Williams, D. S. Mills, and K. Guo. "Development of a head-mounted, eye- tracking system for dogs". In: Journal of Neuroscience Methods 194.2 (2011), pp. 259-265. DOI: 10.1016/j.jneumeth.2010.10.022 (cit. on pp. 23, $36-38)$.

[358] A. D. Wilson and H. Benko. "Combining multiple depth cameras and projectors for interactions on, above and between surfaces". In: Proceedings of the 23nd annual ACM symposium on User interface software and technology - UIST '10. New York, New York, USA: ACM Press, 2010, pp. 273-282. DOI: 10.1145/1866029.1866073 (cit. on p. 168).

[359] C. A. Wingrave, J. Rose, T. Langston, and J. J. J. LaViola. "Early explorations of CAT: canine amusement and training". In: CHI '10 Extended Abstracts on Human Factors in Computing Systems. 2010, pp. 2661-2669. DOI: $10.1145 / 1753846.1753849$ (cit. on pp. $23,30,57,63,81,99,122,123$ ).

[360] M. Winters et al. "Knowledge engineering for unsupervised canine posture detection from IMU data". In: Proceedings of the 12th International Conference on Advances in Computer Entertainment Technology - ACE '15. New York, New York, USA: ACM Press, 2015, pp. 1-8. DOI: 10.1145/2832932. 2837015 (cit. on pp. 22, 23, 29, 99, 113, 122, 131).

[361] H. Wirman. "Games for/with strangers - captive orangutan (Pongo Pygmaeus) touch screen play". In: Antennae 30 (2014), pp. 105-115 (cit. on pp. 23, 26, 34, 42, 45, 99, 198, 222, 226, 254). 
[362] H. Wirman. "Orangutan play on and beyond a touchscreen". In: Proceedings of the 19th International Symposium on Electronic Art, ISEA2013, Sydney. 2013 (cit. on pp. 149, 224).

[363] H. Wirman and A. Zamansky. "Toward characterization of playful ACI". In: Interactions 23.4 (2016), pp. 47-51. DOI: 10.1145/2948127 (cit. on pp. 4 , $5,16,96,97,198,222)$.

[364] H. E. Wirman and I. K. H. Jørgensen. "Designing for intuitive use for non-human users". In: Proceedings of the 12th International Conference on Advances in Computer Entertainment Technology - ACE '15. New York, New York, USA: ACM Press, 2015, pp. 1-8. DOI: 10.1145/2832932.2837008 (cit. on pp. 5, 23, 34, 99, 148, 149, 155, 159).

[365] J. O. Wobbrock, M. R. Morris, and A. D. Wilson. "User-defined gestures for surface computing". In: Proceedings of the 27th international conference on Human factors in computing systems - CHI 09. New York, New York, USA: ACM Press, 2009, pp. 1083-1092. DOI: 10.1145/1518701.1518866 (cit. on pp. 171, 172, 179).

[366] A. Yilmaz, O. Javed, and M. Shah. "Object tracking". In: ACM Computing Surveys 38.4 (2006), 13-es. DOI: 10.1145/1177352.1177355 (cit. on p. 158).

[367] K. Yonezawa, T. Miyaki, and J. Rekimoto. "Cat@Log: sensing device attachable to pet cats for supporting human-pet interaction". In: Proceedings of the International Conference on Advances in Computer Enterntainment Technology - ACE '09. New York, New York, USA: ACM Press, 2009, pp. 149-156. DOI: 10.1145/1690388.1690414 (cit. on pp. 23, 29, 81, 122, 123).

[368] J. Young, N. Young, S. Greenberg, and E. Sharlin. Feline Fun Park: A Distributed Tangible Interface for Pets and Owners. 2013 (cit. on p. 63).

[369] C. Zeagler et al. "Canine computer interaction: towards designing a touchscreen interface for working dogs". In: Proceedings of the Third International Conference on Animal-Computer Interaction - ACI '16. New York, New York, USA: ACM Press, 2016, pp. 1-5. DOI: 10.1145/2995257.2995384 (cit. on pp. 16, 19, 21-23, 33, 35, 42).

[370] C. Zeagler et al. "Going to the dogs: Towards an Interactive Touchscreen Interface for Working Dogs". In: Proceedings of the 27th annual ACM symposium on User interface software and technology - UIST' 14. New York, New 
York, USA: ACM Press, 2014, pp. 497-507. DOI: 10.1145/2642918.2647364 (cit. on pp. 23, 35).

[371] C. Zeagler et al. "Search and rescue: dog and handler collaboration through wearable and mobile interfaces". In: Proceedings of the Third International Conference on Animal-Computer Interaction - ACI '16. New York, New York, USA: ACM Press, 2016, pp. 1-9. DOI: 10.1145/2995257.2995390 (cit. on pp. 23, 28, 121).

[372] J. Zimmerman, J. Forlizzi, and S. Evenson. "Research through design as a method for interaction design research in HCI". In: Proceedings of the SIGCHI conference on Human factors in computing systems - CHI 'O\%. New York, New York, USA: ACM Press, 2007, pp. 493-502. DOI: 10.1145/ 1240624.1240704 (cit. on pp. 7, 254).

[373] J. Zimmerman, E. Stolterman, and J. Forlizzi. "An analysis and critique of Research through Design". In: Proceedings of the 8th ACM Conference on Designing Interactive Systems - DIS '10. New York, New York, USA: ACM Press, 2010, pp. 310-319. DOI: 10.1145/1858171.1858228 (cit. on p. 254).

[374] ZOO Boomer balls. https://www.youtube.com/watch?v=Od_Lm8U5W44. [Online; accessed 16-April-2018]. 2013 (cit. on p. 92). 



\section{List of Figures}

1.1 Problem decomposition (in rectangles) and the chapters in which the subjects are dealt with (in circles). . . . . . . . . 8

2.1 Representation of the gulf of execution in ACI systems. . . . . . . 20

2.2 Framework for technologies in ACI (building from Jukan et al.

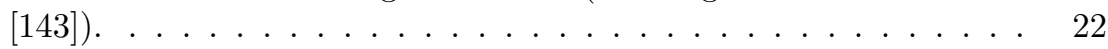

2.3 Button system used as a pressure plate to dispense treats [89]. . . 24

2.4 A dog activating a switch [181]. Photo courtesy of The Open Uni-



2.5 Posture system used by Majikes et al. [171] . . . . . . . . . . . . . 30

2.6 Olfaction cancer detection system [137]. Photo courtesy of The Open University. . . . . . . . . . . . . . . . . 32

2.7 Dog training to click on points on a touchscreen interface [369]. . . 33

2.8 Apps for Apes: An orangutan using a touchscreen [342] . . . . . . . 34

2.9 Human and orangutan playing together with a touchscreen interface

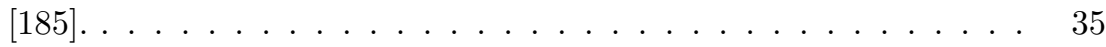

2.10 Using a head-mounted, eye-tracking system with dogs [357]. . . . . 38

2.11 Tracking cats using depth measurement via an Xbox Kinect to detect posture $[257] . \ldots \ldots \ldots \ldots$

2.12 Tracking dogs using posture recognition via an Xbox Kinect [190]. 40

3.1 Dog playing with a Sphero . . . . . . . . . . . . . 50 
3.2 Orangutans playing with an iPad as part of the Apps for Apes ini-

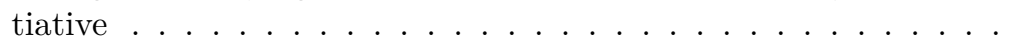

3.3 Chimpanzee Lana using the lexigram keyboard to request food (Image courtesy of. Dr. Duane Rumbaugh) . . . . . . . . . . . .

3.4 Chicken wearing a jacket which simulates human touching sensation (Image courtesy of Dr. Adrian David Cheok) . . . . . . . . . . . 54

3.5 Diabetes Alert Dog using a prototype of the alert device used to communicate with emergency services (Image courtesy of Dr. Clara Mancini and Charlotte Robinson) . . . . . . . . . . . . . . 55

3.6 Dog using the cancer detection interface (Image courtesy of Dr. Clara Mancini . . . . . . . . . . . . . . . . . 55

3.7 LonelyDog@Home graphical interface for pet owners (Image courtesy of LonelyDog@Home's authors) . . . . . . . . . . . . . . . 57

3.8 Cats playing with Felino (Images courtesy of Michelle Westerlaken) 59

3.9 Problem decomposition of the work described in Part II. . . . . . . 77

4.1 Set-up for the tracking system. . . . . . . . . . . . . . 83

4.2 Process of extracting the cat's orientation: (a) color frame (b) depth frame, (c) background segmentation, (d) cat contours, (e) clusters for head, body and tail, (f) orientation vector. . . . . . . . . . 83

4.3 Cat running after a Sphero ${ }^{\circledR}$ robot. . . . . . . . . . . . . . 85

4.4 Human postures and gestures during play: a) sitting b) kneeling c) sideways d) crouching down. . . . . . . . . . . . . . 86

4.5 a) and b) Correct classification c) and d) erroneous classification due to ambiguous posture. . . . . . . . . . . . . . 88

4.6 Orientation vectors differ from the heads' current orientation. . . . 88

4.7 Tiger playing with giant plastic ball (image courtesy of Boomer Ball). 93

4.8 Human using the Virtuix Omni platform (image courtesy of Virtuix). 93

5.1 Set-up of the observational study: play area and location of the projector and depth sensor. . . . . . . . . . . . . . 102 
5.2 Sphero (left) and Jumping Sumo (right), the two robots used in the study. . . . . . . . . . . . . . . 103

5.3 Different paths of the mice in Activity 4. . . . . . . . . . . . . 105

5.4 Two kittens playing with a Sphero ${ }^{\circledR}$. . . . . . . . . . . . . . 106

5.5 Process of extracting the cat's orientation and body parts: (a) color frame (b) depth frame, (c) background segmentation, (d) cat contours, (e) clusters for head, body and tail, (f) orientation vector. . 110

5.6 Color, depth and clustered frames for different cats' postures (a) standing (b) walking (c) sitting (d) semi-sitting (e) turning (f) jumping. . . . . . . . . . . . . . . . . . . . . .

6.1 Set-up for the tracking system. . . . . . . . . . . . .

6.2 Process of extracting the cat's orientation: (a) color frame (b) depth frame, (c) background segmentation, (d) cat contours, (e) clusters for head, body and tail, (f) orientation vector. . . . . . . . . . .

6.3 Cat's postures and the corresponding depth and clustered image (a) standing (b) walking (c) sitting (d) turning (e) semisitting (f) jumping. . . . . . . . . . . . . . . 132

6.4 Kitten chasing a Sphero ${ }^{\circledR} \ldots \ldots$. . . . . . . . . . . . 138

6.5 Problem decomposition of the work described in Part III. . . . . . 145

8.1 Orangutan using objects to interact with projection . . . . . . 157

8.2 Problem decomposition of the work described in Part IV. . . . . . 165

9.1 Sketch of the Wizard-of-Oz set-up. . . . . . . . . . . . . . 175

9.2 Path from Task 2. . . . . . . . . . . . . . . . 176

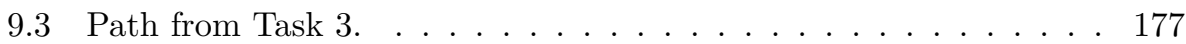

9.4 Time performance (seconds) for each task by age and group gender. 179

9.5 Taxonomy distribution according to age groups. . . . . . . . . . . . 181

9.6 Taxonomy distribution according to gender. . . . . . . . . . . . 182 
9.7 Distribution on the use of body parts for different actions by age



9.8 Distribution of types of gestures by action and age group. . . . . . 183

9.9 Example of robot-centric and user-centric gestures. . . . . . . . . . 185

9.10 Gestural language with most frequent gestures for each action and occurrence frequencies. . . . . . . . . . . . . 187

9.11 Results for Q1 in the postquestionnaire by participant group and gender. . . . . . . . . . . . . . 188

9.12 Results for Q2 in the postquestionnaire by participant group and gender. . . . . . . . . . . . . . . 188

9.13 Results for Q3 in the postquestionnaire by participant group. . . . 189

9.14 Results for Q4 in the postquestionnaire by participant group. . . . 189

9.15 Results for Q5 in the postquestionnaire by participant group. . . . 190

9.16 Results for Q5 in the postquestionnaire by gender and participant group. . . . . . . . . . . . . . . . . . 191

9.17 Results for Q6 in the postquestionnaire by participant group and gender. . . . . . . . . . . . . . . . . . 191

10.1 Cutouts used in the design activities. . . . . . . . . . . . . . 202

10.2 Examples of different physical locations and boundaries. . . . . . . 207

10.3 Physical boundaries in the designs. . . . . . . . . . . . . 208

10.4 a) Race between a snake and $\mathrm{RC}$ car, b) Child using a drone to fed the crocodile, c) Eagle playing with drone controlled by child. . . . 209

10.5 Matching of physical traits. . . . . . . . . . . . . . 210

10.6 Degree of participation. . . . . . . . . . . . . 211

10.7 a) Three children playing with three fish, b) Animals observing, c) Several animals playing. . . . . . . . . . . . . . . 211

10.8 a) Child racing with a gorilla to get a banana, b) Child and cat playing to untangle yarn balls, c) Translation app to speak with an elephant. . . . . . . . . . . . . . . . . 214 
10.9 Postquestionnaire answers to perceived human and animal fun by category and type of animal. . . . . . . . . . . . . 215

10.10Pre and post questionnaire answers to perceived aspects for the different animal categories. . . . . . . . . . . . . . 216

11.1 System deployment and set-up. . . . . . . . . . . . . . . . 227

11.2 Hospitalized child controlling a Sphero ${ }^{\circledR}$ to remotely play with a dog in a canine daycare facility. . . . . . . . . . . . . . 228

11.3 Communication between applications. . . . . . . . . . . . . 229

11.4 Example of the 5-point Likert scale answer options. . . . . . . . . . 229

11.5 Postquestionnaire results from the Likert scale questions. . . . . . 232

11.6 Play area for the study. . . . . . . . . . . . . . 236

11.7 a) Color stream; b) Detection of dog and robot contours based on movement. . . . . . . . . . . . . . 237

11.8 a) Dog interacting with robot; b) Dog not paying attention to the

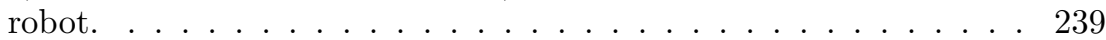





\section{List of Tables}

2.1 Classification of Interactive Technologies in Animal Computer Interaction. .................. . . 23

3.1 List of requirements and features of intelligent playful environments 62

3.2 Analysis of existing playful games for animals under the proposed

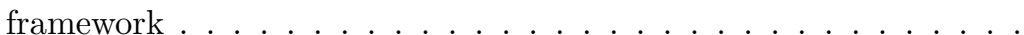

4.1 Accuracy of the tracking system identifying the cats' body postures and orientation vector.

5.1 Different cats' postures observed during playful activities. . . . . . 109

5.2 Classification of cats' body parts using a supervised approach. . . . 113

5.3 Classification of cats' body postures using a supervised approach. . 113

5.4 Classification of cats' body parts and postures using a knowledgebased approach. . . . . . . . . . . . . . . . . 113

6.1 Existing wearable tracking systems for animals and summary of features. . . . . . . . . . . . . . . . . 123

6.2 Accuracy rates for base learners when classifying a cluster belonging to a cat's contour. . . . . . . . . . . . . . . . 129

6.3 Accuracy rates for base learners when classifying a cat's posture. . 130

6.4 Accuracy rates for base learner when classifying a cat's posture, using forward feature selection. . . . . . . . . . . 130 
6.5 Accuracy of the tracking system identifying the cats' postures and body parts. ...................... 133

7.1 Examples of tangible-mediated activities. . . . . . . . . . . . 150

9.1 List of actions (referents) the mini-drone can perfom. . . . . . . 176

9.2 Postquestionnaire and answer options. . . . . . . . . . . . . . . 178

9.3 Time performance (seconds) for each task by age group and gender. 178

9.4 Gesture taxonomy. . . . . . . . . . . . . . . . . 180

9.5 Agreement level of gestures for the different actions proposed in T1 by participant group. . . . . . . . . . . . . 186

10.1 Classification of animals presented in the study. . . . . . . . . . 203

10.2 Questions before the game design activity. . . . . . . . . . . 203

10.3 Questions to be asked before and after the game design activity. . 204

10.4 Questions after the game design activity. . . . . . . . . . . . 204

11.1 Postquestionnaire 5-point Likert questions and open answer question.230

11.2 Mode, mean and standard deviation of children's answers in the postquestionnaire. . . . . . . . . . . . . . 231 